Universidad Politécnica de Madrid

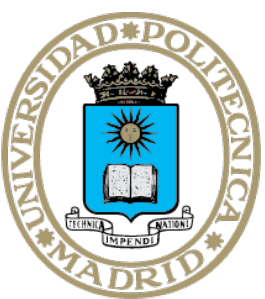

\title{
POLITÉCNICA
}

Departamento de Tecnología Fotónica y Bioingeniería

Escuela Técnica Superior de Ingenieros de Telecomunicación
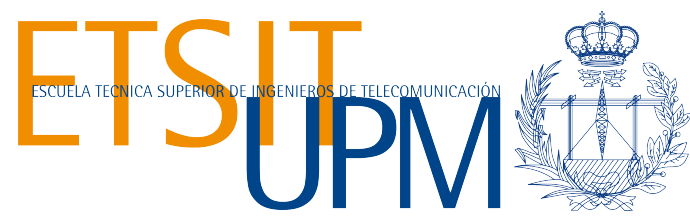

\section{Análisis dimensional conforme a la dinámica y complejidad de las señales biológicas}

\author{
AUTOR \\ Javier de Pedro Carracedo \\ Ingeniero de Telecomunicación
}

DIRECTORA

Ana P. González Marcos

Dra. Ingeniero de Telecomunicación 

D. a Ana Pilar González Marcos, catedrática de la Universidad Politécnica de Madrid (UPM), certifica que la memoria titulada

\section{Análisis dimensional conforme a la dinámica y complejidad de} las señales biológicas

fue realizada por Javier de Pedro Carracedo bajo su dirección y tutoría, y que el interesado se encuentra en condiciones de optar al grado de Doctor, por lo que solicita que sea admitida a trámite para su lectura y defensa pública.

Ámbito de conocimiento: Ingeniería biomédica.

Madrid, marzo de 2020

Fdo.: Ana P. González Marcos

(Directora y tutora) 



\section{TRIBUNAL CALIFICADOR}

Tribunal nombrado por el Excmo. y Magfco. Sr. Rector de la Universidad Politécnica de Madrid el día de de

Presidente Dr./Dra. D./D. ${ }^{\mathrm{a}}$

Vocal Dr./Dra. D. $/$ D. $^{\mathrm{a}}$

Vocal Dr./Dra. D./D. ${ }^{a}$

Vocal Dr./Dra. D. $/$ D. $^{\mathrm{a}}$

Secretario Dr./Dra. D./D. ${ }^{a}$

Realizado el acto de lectura y defensa de la Tesis el día de de en Madrid.

Calificación:

EL PRESIDENTE

LOS VOCALES

EL SECRETARIO 

A mis padres, en especial a mi difunto padre, con seguridad en manos de Dios, y a mi sobrino y ahijado Álvaro 



\section{AGRADECIMIENTOS}

Llegado a este punto son muchos los retazos mentales que se me proyectan en la consciencia. El fallecimiento de mi padre, acaecida recientemente, evoca en mi memoria momentos inolvidables. Con su muerte todo cobra sentido. Sin saberlo, o quizás sí lo sabía en su fuero interno, me había transmitido, de la mano de mi madre, los valores que a día de hoy considero de incalculable trascendencia, pues son los que han forjado lo que soy y no tanto lo que tengo; son los que van desterrando las apariencias en favor de la esencia; son los que apuestan por la sencillez, el esfuerzo lento y paciente y la mesura en el trato con los demás. Creo firmemente que la aleación de todas estas piedras preciosas, la herencia velada de mis padres, constituye el material de construcción del trirreme con el que se emprendió esta ardua misión para que finalmente recalara en buen puerto. Por todo ello mi primer y merecido agradecimiento no puede ser otro que honrar a mis padres. Nunca podré corresponderles en su justa medida.

Continúan mis gratificaciones con el prócer Raúl Durán Díaz, de la Universidad de Alcalá e investigador del Consejo Superior de Investigaciones Científicas (CSIC). Durante todo el tiempo de vida de este trabajo siempre ha estado ahí, aleccionándome en el cuidado de los detalles y en el inflexible respeto por la rigurosidad del quehacer diario. El vago recuerdo de mi época universitaria se ha hecho más nítido. La disciplina y la constancia han vuelto a recuperar su lugar de honor merced a las lecturas que Raúl compartía conmigo, como La vida intelectual, de Antonin-Dalmace Sertillanges, o El amor a la sabiduría, de Étienne Gilson, entre otras. Estas lecturas, junto con las recomendaciones que me procuraba, en materia de teoría de los sistemas dinámicos, actuando de mediador con su superior en el CSIC, D. Jaime Muñoz Masqué, todo un referente en la sombra, se convirtieron en la guarnición de mi voluntad para poder afrontar este desafío. Además, me facilitó un cluster de servidores con los que he podido ejecutar algoritmos cuya carga computacional, de otro modo, hubiera supuesto un retraso temporal innecesario. Mi reconocimiento por tamaña asistencia va más allá de estas breves líneas.

Quisiera expresar un agradecimiento especial por los autores de Nonlinear Time Series Analysis, Holger Kantz y Thomas Schreiber, un libro de obligada lectura en el análisis de sucesiones temporales. La experiencia acumulada en el libro y el software TISEAN ${ }^{\complement}$, que acompañan, me han permitido no solo adquirir el bagaje teórico que demanda esta materia sino también la práctica experimental que conlleva operar con el software que estos mismos autores, junto con la ayuda de Rainer Hegger, amablemente ponen a disposición de todos aquellos que quieran iniciarse en estas lides.

Hago extensivo este encadenamiento de agradecimientos a compañeros del Departamento de Automática, de la Universidad de Alcalá, que de una u otra forma, en muchos casos inconscientemente, han contribuido a la consecución de este trabajo. Así, reciban una particular mención Juan Ignacio Pérez Sanz, por su apoyo en la terminología inglesa, Julia Clemente Párraga, por su perseverancia en todo lo que 
hace, Melquíades Carbajo Martín, por su desenfadada brillantez, y Francisco Márquez García, por su tesón en el estudio al margen de las recompensas. Todos ellos son ejemplos de lo que debería ser la verdadera universidad, de ahí mi más profunda y silenciosa admiración. Lamentablemente la universidad actual camina por otros derroteros.

En esta serie de agradecimientos no me olvido de mi tutora Ana González Marcos, quien me ha brindado la oportunidad, apostando por mí más que mi propia persona, de abordar con ella esta tarea investigativa. Desde hace bastantes años ha estado insistiendo incansablemente para que pudiera cerrar esta etapa académica. Ella ha colocado la cuña pragmática a un idealismo ácrono. Sinceramente, aún no me lo puedo creer. He de reconocer que no fue la única línea de investigación que se emprendió y muchas otras quedaron a mitad de camino; aun así, nunca dejó de creer en mí. Mi gratitud hacia ella trasciende lo meramente profesional.

Finalmente concluyo este apartado con un broche de oro, Emma Castelló Taliani, la half-bubble, como así la llamo. Como pilar fundamental de mi vida es todo inspiración y un orgullo para mí, pues encarna los valores de una persona de altura tanto en lo personal como en lo profesional, con una educación exquisita. Sin ella este trabajo no hubiera llegado a su fin y estas palabras no quedarían impresas en esta memoria. 


\begin{abstract}
The characterization of the dynamic behavior of biological signals has played, for several decades now, an essential role in early diagnostics of pathologies, both physical and emotional. This work outlines a methodology which, based on a scalar time series obtained experimentally, something quite usual in the clinical field, allows the reconstruction of the space of states which describe the functional response of a physiological process. This methodology relies on very consolidated mathematical foundations, the theory of nonlinear dynamic systems. Once the space where the dynamics of the physical process develops has been reconstructed, we are in a situation where we can characterize its spatial or structural complexity, from a geometric and temporal viewpoint, as it evolves in time. Among outstanding complexity metrics, some stand out, such as entropy, fractal dimension and Lyapunov exponents which, with their many deductive variants, allow us to evaluate the degree of regularity which repetitive patterns present in the time series experiment, and to estimate the structural richness which describes its dynamics.

In the context of this research, we undertake the study of a specific biological signal, the PPG (PhotoPlethysmoGraphic) signal, attending to the outlined methodology and according to the most appropriate nonlinear analysis tools. The characterization of its functional response, in contrast to other fundamental reference signals whose behavior is known a priori, allows us to identify its dynamic complexity and, therefore, estimate how the index of coupling between the subsystems which make up the functionality of the underlying physiological process evolves. In this analytical frame it would be feasible to establish a protocol to compare PPG signals from individuals subjected to a stressful situation, with respect to their counterparts in the basal state, so that we can figure out what stress results in, from an operative perspective, according to the functional response of the physiological process which triggers it, and how the organism reestablishes the optimal working conditions or, in a more adverse scenario, how it dynamically expresses a possible irreversible degradation of the system.
\end{abstract}




\section{RESUMEN}

La caracterización del comportamiento dinámico de las señales biológicas ha desempeñado, desde hace varias décadas, un papel esencial en el diagnóstico temprano de patologías tanto físicas como emocionales. Este trabajo delinea una metodología que, sobre la base de una sucesión temporal escalar adquirida experimentalmente, algo muy habitual en el ámbito clínico, propicia la reconstrucción del espacio de estados que describen la respuesta funcional de un proceso fisiológico. Esta metodología descansa sobre una fundamentación matemática bastante consolidada, la teoría de los sistemas dinámicos no lineales. Una vez reconstruido el espacio en el que se desenvuelve la dinámica del proceso físico, se está en condiciones de poder caracterizar su complejidad espacial o estructural, desde un punto de vista geométrico, y temporal, a medida que aquella evoluciona con el tiempo. Entre las medidas de la complejidad sobresalen parámetros como la entropía, la dimensión fractal y los exponentes de Lyapunov, que, en sus múltiples variantes deductivas, facilitan la evaluación del grado de regularidad que experimentan los patrones repetitivos presentes en la sucesión temporal y la estimación de la riqueza estructural que describe su dinámica.

En el contexto de esta investigación se acomete el estudio de una señal biológica en particular, la señal PPG, del inglés photoplethysmographic, atendiendo a la metodología planteada y de acuerdo con las herramientas de análisis no lineal más oportunas. La caracterización de su respuesta funcional, en contraste con otras señales de referencia básicas cuyo comportamiento es conocido a priori, permite identificar su complejidad dinámica y, por tanto, estimar cómo se desarrolla el índice de acoplamiento entre los subsistemas que conforman la funcionalidad del proceso fisiológico subyacente. Con este marco analítico resultaría factible establecer un protocolo de actuación con el que comparar señales PPG de sujetos sometidos a una situación de estrés, respecto a sus contrapartes en estado basal, de manera que pueda descifrarse en qué se traduce el estrés desde una perspectiva operativa, a tenor de la respuesta funcional del proceso fisiológico que la desencadena, y cómo el organismo restablece las condiciones óptimas de funcionamiento o, en un escenario más adverso, cómo exterioriza dinámicamente una posible degradación irreversible del sistema. 


\section{Índice de contenidos}

1 Introducción $\quad 23$

2 Sistemas dinámicos $\quad 29$

2.1 Breve panorámica histórica . . . . . . . . . . . . . . . 29

2.2 Historia de los sistemas dinámicos f . . . . . . . . . . . . . . 31

2.3 Sistemas dinámicos disipativos, recurrentes y ergódicos . . . . . . . . 34

2.3 .1 Sucesiones temporales y observables . . . . . . . . . 36

2.3.2 Dinámica de un sistema a partir de una sucesión temporal . . 37

2.3.2.1 Teorema ergódico de Birkhoff . . . . . . . . . . . 37

2.3.2.2 Teorema de reconstrucción de Floris Takens . . . . . 38

$\begin{array}{ll}\text { Referencias } & 41\end{array}$

3 Reconstrucción del espacio de estados. Método de los retardos 45

3.1 El porqué de la reconstrucción de estados . . . . . . . . . . . . 47

3.1.1 Inconvenientes en un escenario real: proceso de reconstrucción 48

3.1 .2 Métodos de reconstrucción . . . . . . . . . . . . . . . 52

3.2 Método de los retardos . . . . . . . . . . . . . . . . . 54

3.2.1 Selección del parámetro $\tau$. . . . . . . . . . . . . . 55

3.2.1.1 Método de llenado del espacio . . . . . . . . . 55

3.2.1.2 Función de autocorrelación . . . . . . . . . . 57

3.2.1.3 Información mutua . . . . . . . . . . . 57

3.2.2 Selección del parámetro $m$. Dimensión de reconstrucción . . . 60

3.2.2.1 Análisis de componentes principales . . . . . . . . 60

3.2.2.2 Método de los falsos vecinos más cercanos . . . . . . 63

3.2.2.3 La suma de correlación. Dimensión de correlación . 65

3.3 Otros métodos de estimación de $\tau$ y $m \ldots \ldots . \ldots . \ldots 72$ 


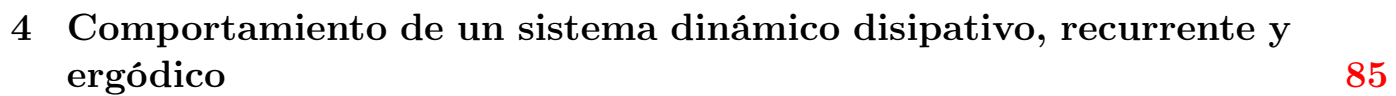

4.1 Bifurcaciones y rutas al caos . . . . . . . . . . . . . 87

4.1 .1 Bifurcaciones . . . . . . . . . . . . . 87

4.1.1.1 Bifurcación tangente (saddle-node bifurcation) . . . 88

4.1.1.2 Bifurcación transcrítica (transcritical bifurcation) . . 89

4.1.1.3 Bifurcación horca (pitchfork bifurcation) . . . . . . 89

4.1.1.4 Bifurcación de Hopf (Hopf bifurcation) . . . . . . . . 90

4.1 .2 Rutas al caos . . . . . . . . . . . . . . . . . . . 91

4.1.2.1 Doblamiento de período . . . . . . . . . . . 92

4.1.2.2 Intermitencia . . . . . . . . . . . . . . . . 93

4.1.2.3 Transición de la cuasiperiodicidad al caos . . . . . . 95

4.2 Grado de complejidad: caracterización de un sistema dinámico . . . . 97

4.2 .1 Exponentes de Lyapunov . . . . . . . . . . . . . . . . . . 99

4.2.1.1 Cálculo de los exponentes de Lyapunov a partir de una sucesión temporal . . . . . . . . . . . . . . 103

4.2 .2 Dimensiones . . . . . . . . . . . . . . . . . . . . . . . . . 112

4.2.2.1 Clasificación de las dimensiones . . . . . . . . . . . 115

4.2.2.2 Dimensión fractal en sucesiones temporales . . . . . 117

4.2.2.3 Algoritmos para computar la dimensión . . . . . . . 120

4.2 .3 Entropía . . . . . . . . . . . . . . . . . . . 123

4.2.3.1 Entropías de una distribución estática . . . . . . . 125

4.2.3.2 Entropía de Kolmogórov-Sinái . . . . . . . . . . . 126

4.2.3.3 Evolución temporal de la entropía . . . . . . . . . . 128

4.2.3.4 Entropías a partir de una sucesión temporal . . . . . 130

4.2.3.5 Relaciones entre los exponentes de Lyapunov, las dimensiones y las entropías . . . . . . . . . . . 132

4.3 Técnicas de medida de la complejidad . . . . . . . . . . . . . . . 134

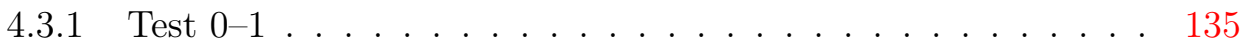

4.3.1.1 Descripción del test . . . . . . . . . . . . 135

4.3.1.2 Descripción del algoritmo . . . . . . . . . . 136

4.3 .2 Complejidad de Lempel-Ziv . . . . . . . . . . . . . . . . 138

4.3.2.1 Descripción del algoritmo . . . . . . . . . . . . . 140

$\begin{array}{lr}\text { Referencias } & 143\end{array}$

5 Señales biológicas $\quad 153$

5.1 Introducción a las señales biológicas . . . . . . . . . . . . . 153 
5.1 .1 Señales biológicas . . . . . . . . . . . . . . . . 155

5.2 Comportamientos dinámicos en fisiología . . . . . . . . . . . 156

5.3 Análisis no lineal de datos fisiológicos . . . . . . . . . . . . . . 160

5.3 .1 Ley de evolución dinámica . . . . . . . . . . . . . . . . 165

5.3 .2 Cómo evaluar la no linealidad . . . . . . . . . . . . . . 167

5.3.2.1 Evaluación gráfica . . . . . . . . . . . . . . 168

5.3.2.2 Test estadístico . . . . . . . . . . . . . . . . . 171

5.3.2.3 Dinámica simbólica . . . . . . . . . . . . . 177

5.4 Un ejemplo de señal biológica: la señal PPG . . . . . . . . . . . . . . 177

5.4.1 La onda de pulso cardiovascular y sus características . . . . . 180

$5.4 .2 \quad$ El estrés . . . . . . . . . . . . . . . . . . . . . . . . . . 184

$\begin{array}{ll}\text { Referencias } & 187\end{array}$

6 Resultados obtenidos 197

6.1 Reconstrucción del espacio de estados . . . . . . . . . . . . . . . 198

6.1.1 Selección del parámetro $\tau$. . . . . . . . . . . . . . . . . . . 199

6.1 .2 Selección del parámetro $m \ldots$. . . . . . . . . . . . . 200

6.2 Grado de complejidad . . . . . . . . . . . . . . . . . . 203

6.2 .1 Exponentes de Lyapunov . . . . . . . . . . . . . . . 203

6.2 .2 Dimensiones . . . . . . . . . . . . . . . . . . . 206

6.2.2.1 Dimensión fractal . . . . . . . . . . . . . 207

6.2.2.2 Dimensiones de información y de correlación . . . . 208

6.2 .3 Entropías . . . . . . . . . . . . . . . . . . . . 211

6.2.3.1 Entropía de dispersión . . . . . . . . . . . . . . . 211

6.2.3.2 Entropía aproximada . . . . . . . . . . . . 212

6.2.3.3 Entropía de muestra multiescala . . . . . . . . . . 214

6.2.3.4 Entropía de permutación . . . . . . . . . . 217

6.3 Técnicas de medida de la complejidad . . . . . . . . . . . . 220

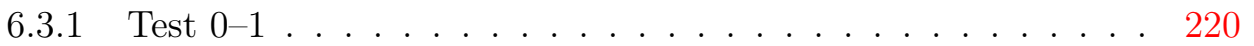

6.3 .2 Complejidad de Lempel-Ziv . . . . . . . . . . . . . . . . . 222

$\begin{array}{ll}\text { Referencias } & 227\end{array}$

7 Conclusiones y líneas abiertas 233

7.1 Líneas abiertas . . . . . . . . . . . . . . . . . . . . 235 
Apéndices

A Señales de referencia y equipamiento destinado

A.1 Señales de referencia . . . . . . . . . . . . . . . . . . . 239

A.1.1 Señales de referencia básicas . . . . . . . . . . . . . . . . . . 239

A.1.2 Señales PPG . . . . . . . . . . . . . . . . . . . 240

A.2 Equipamiento destinado . . . . . . . . . . . . . . . . . 240

A.2.1 Entorno de desarrollo software . . . . . . . . . . . . . 240

A.2.2 Infraestructura hardware . . . . . . . . . . . . . . 241

B Publicaciones en revistas indexadas 243 


\section{Índice de figuras}

1.1 Diagrama de flujo de las dependencias conceptuales entre los capítulos. 24

3.1 Planteamiento del problema de reconstrucción del espacio de estados. 46

3.2 La amplificación del ruido y la estimación del error en dos escenarios ilustrativos [eje de abscisas, estado del sistema en el instante de tiempo $t$; eje de ordenadas, estado en el instante $t+t_{1}$ ]. En (a) el grosor de la distribución de puntos es sintomático de una amplificación de ruido significativa; en (b) la dinámica funcional sinuosa de los puntos es más compleja, lo que, en presencia de pocos puntos, podría llevar a errores de predicción notorios. . . . . . . . . . . . . . 50

3.3 Espacio de estados del atractor de Lorenz. . . . . . . . . . . . . . . . 54

3.4 Reconstrucción del espacio de estados a partir de la variable dinámica $x$ del atractor de Lorenz para diferentes valores de $\tau$. Puede observarse cómo a medida que aumenta $\tau$ el atractor va desplegándose en toda su magnitud hasta un punto en que se desvanece toda causalidad dinámica. 56

3.5 Función de autocorrelación del atractor de Lorenz para la variable dinámica $x$, en la que se indica el primer paso por cero, cuando $\tau=409.57$

3.6 Información mutua del atractor de Lorenz para la variable dinámica $x$, en la que se indica el primer mínimo, cuando $\tau=16 . \ldots$. . . .

3.7 Análisis de componentes principales del atractor de Lorenz para la variable dinámica $x$; en el recuadro interior se aprecia claramente cómo con solo tres direcciones puede caracterizarse el comportamiento dinámico del atractor. . . . . . . . . . . . . . . . . . . . .

3.8 Falsos vecinos más cercanos del atractor de Lorenz para la variable dinámica $x$. A partir de $m=3$ el número de falsos vecinos más cercanos se reduce a cero. . . . . . . . . . . . . . . . .

3.9 Dimensión de correlación del atractor de Lorenz para la variable dinámica $x$. A partir de $m=3$ la dimensión de correlación se confina a un valor constante en un rango intermedio y reducido de escalas $\varepsilon . \quad$. 66

3.10 Regiones características de la suma de correlación. . . . . . . . . . . 67

4.1 Bifurcación tangente de la ecuación $\dot{x}=r+x^{2} \ldots \ldots \ldots 8$

4.2 Bifurcación transcrítica de la ecuación $\dot{x}=r-x^{2}$. . . . . . . . . 89

4.3 Bifurcación horca de la ecuación $\dot{x}=r x-x^{3} \ldots \ldots \ldots$. . . . . 89

4.4 Bifurcación horca de la ecuación $\dot{x}=r x+x^{3}$. . . . . . . . . . . 90

4.5 Bifurcación bidimensional de Andronov-Hopf, en su variante supercrítica. . . . . . . . . . . . . . . . . . 9 90 
4.6 Diagrama de bifurcación del mapa logístico $x_{n+1}=r x_{n}\left(1-x_{n}\right)$ para

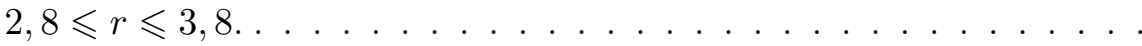

4.7 Intermitencia del mapa logístico $x_{n+1}=r x_{n}\left(1-x_{n}\right)$, cuando el pará-

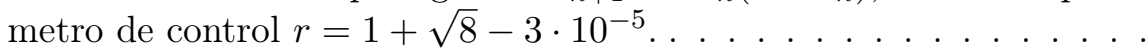

4.8 Intermitencia, con representación de las iteraciones, del mapa logístico $f(x)=r x(1-x)$, cuando el parámetro de control $r=1+\sqrt{8}-3 \cdot 10^{-5}$, y el valor inicial $x_{0}=0,5 \ldots \ldots \ldots \ldots \ldots$

4.9 Ejemplo de transición desde la cuasiperiodicidad al caos en el caso del mapa seno $\theta_{n+1}=\theta_{n}+\Omega-(k / 2 \pi) \sin \left(2 \pi \theta_{n}\right)$ (mód 1$)$, para $k \geqslant 0$. En (a) se obervan claramente las órbitas periódicas (hilos de Arnold); en (b) los hilos de Arnold se desdoblan, multiplicando su periodicidad o volviéndose caóticos. . . . . . . . . . . . . . . . . . . .

4.10 Fenómenos de expansión y contracción de una unidad de volumen infinitesimal $\bullet$ describiendo una trayectoria en un espacio de estados.

4.11 Constructo de la estimación del mayor exponente de Lyapunov a partir de la sucesión temporal. En cada instante de tiempo $t_{j}$, en que se efectúa el reemplazo de la trayectoria vecina, han de minimizarse el ángulo $\theta_{t_{j}}$ y la distancia al nuevo punto vecino inicial. . . . . . . . .

4.12 Los puntos dentro del círculo $\odot$, para una ventana temporal $w=4$, son considerados vecinos temporales del punto $\bullet$, que identifica un estado de referencia de la trayectoria de interés. . . . . . . . . . . .

4.13 Mapa de desplazamiento de Bernoulli. En (a) se particulariza el procedimiento de estiramiento y plegado; en $\mathbf{1}$ se divide la baraja en dos montones exactamente iguales (el mapa es dos a uno); en $\mathbf{2}$ se curvan las puntas de las dos mitades hacia arriba hasta conseguir una altura que sea el doble que la altura original de cada montón (estiramiento); en 3 se entremezclan los dos montones arbitrariamente (plegado), del modo en que se baraja a la americana. Una vez que se dispone de un único montón, según 4 , se repite el procedimiento tantas veces (iteraciones) como sean necesarias. En (b) se representa la característica onda de sierra de un mapa de desplazamiento de Bernoulli.

4.14 Comportamiento de $h_{q}(m, \varepsilon)$, con $q>0$, para tres diferentes procesos ilustrativos. . . . . . . . . . . . . . . .

4.15 Diagrama de flujo del algoritmo que permite calcular $c(n) \ldots \ldots$. .

5.1 Esquema general de un sistema de adquisición de datos biomédicos. .

5.2 Esbozo de una sección de Poincaré. . . . . . . . . . . . . . . . 167

5.3 Gráfico de recurrencia del atractor de Lorenz para la variable dinámica $x$. La representación gráfica obedece a $m=3, \tau=16$ y $r=5$. . . .

5.4 Gráfico de separación espacio-temporal del atractor de Lorenz para la variable dinámica $x$. Con $m=3$ y $\tau=16$, se muestran las líneas de contorno que representan, para una separación espacial $\varepsilon$ y una separación temporal $\Delta t$, las fracciones de puntos (en $\%) \mathbf{x}_{i} \in \mathbb{R}^{m}$

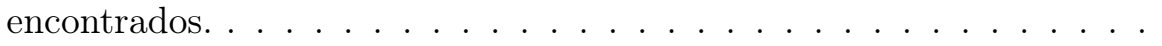

5.5 Forma de onda de una señal PPG típica. . . . . . . . . . . . . . . . 180 
5.6 Pinzamiento de un dedo con el pletismógrafo a fin de capturar la señal PPG de un individuo [imagen disponible públicamente en flickr y adquirida el 30 de octubre de 2018]. . . . . . . . . . . . . .

5.7 Parámetros característicos del pulso PPG. En (a) la amplitud del pico sistólico se denota con el símbolo $\mathbf{A}_{\text {sist }}$ y la amplitud del pico diastólico con el distintivo $\mathbf{A}_{\text {diast }}$. En (b) el intervalo pico a pico entre dos pulsos consecutivos de la señal PPG. . . . . . . . . . . . . . . . . . . .

6.1 Evolución temporal de las señales de referencia (a)-(e) y de las señales PPG (f)-(j), adquiridas de cinco individuos elegidos aleatoriamente. .

6.2 Función de autocorrelación de la señal PPG para el individuo número 4 , en la que se señala el primer paso por cero, cuando $\tau=44$. . . .

6.3 Información mutua de la señal PPG para el individuo número 4, en la que se indica el primer mínimo, cuando $\tau=54$. . . . . . . . . . . .

6.4 Análisis de componentes principales de la señal PPG para el individuo número 4; en el recuadro interior no se aprecia claramente con cuántas direcciones o dimensiones puede caracterizarse el comportamiento dinámico del atractor. . . . . . . . . . . . . . . . . . .

6.5 Falsos vecinos más cercanos de la señal PPG para el individuo número 4. A partir de $m=4$ el número de falsos vecinos más cercanos se reduce prácticamente a cero. . . . . . . . . . . . . . . . .

6.6 Gráfico de separación espacio-temporal de la señal PPG para el individuo número 4 . Como puede advertirse, en torno a $\Delta t \approx 300$ las líneas de contorno mantienen valores aproximadamente constantes. .

6.7 Dimensión de correlación de la señal PPG para el individuo número 4. A partir de $m=3$ la dimensión de correlación se confina a un valor constante en un rango intermedio y reducido de escalas $\varepsilon$. . . . . . .

6.8 Divergencia exponencial de trayectorias inicialmente cercanas entre sí, indicativo de la presencia de un exponente de Lyapunov positivo, y, por tanto, de caos determinista. . . . . . . . . . . . . . .

6.9 Evolución temporal de las señales de referencia para el cómputo de la dimensión fractal. En (a) se presenta una realización de la función coseno de Weierstrass (WCF), de $D_{F}=1,2$; en (b) una realización de la función coseno de Weierstrass-Mandelbrot (WMCF), de $D_{F}=1,3$; en (c) una realización de la función de Takagi (TF), también conocida como función de Knopp, de $D_{F}=1,5$; en (d) una realización de un movimiento browniano fraccionario $(\mathrm{fBm})$, de $D_{F}=1,7 \ldots \ldots$. . 207

6.10 Dimensión de información de la señal PPG. . . . . . . . . . . . . . . 209

6.11 Dimensión de correlación $\left(D_{2}\right)$ de las señales de referencia (a)-(e) y de las señales PPG (f)-(j), adquiridas de cinco individuos elegidos aleatoriamente. . . . . . . . . . . . . . . . 
6.12 Tendencia de la entropía aproximada (ApEn), cuando $r=0,2$, conforme se van acumulando bloques de datos de longitud 500, de las señales de referencia y de las señales PPG, adquiridas de cinco individuos elegidos aleatoriamente. En (a) se muestran todas las señales; en (b) únicamente se reflejan las señales PPG en comparación con aquellas señales que presentan similares tendencias entrópicas en orden de magnitud. . . . . . . . . . . . . . . . .

6.13 Evolución de la entropía de muestra multiescala (multescEn), cuando $r=0,2$, para diferentes factores de escala, de las señales de referencia y de las señales PPG, adquiridas de cinco individuos elegidos aleatoriamente. En (a) se muestran todas las señales; en (b) únicamente se reflejan las señales PPG (su tendencia aparece resaltada) en comparación con aquellas señales que presentan similares tendencias entrópicas en orden de magnitud. . . . . . . . . . . . . . . . . . . .

6.14 Entropía de permutación (permutEn) de las señales de referencia (a)-(e) y de las señales PPG (f)-(j), adquiridas de cinco individuos elegidos aleatoriamente. . . . . . . . . . . . . . .

6.15 Tendencia de la entropía de permutación (permutEn), conforme se van acumulando bloques de datos de longitud 1.000, de las señales de referencia (a)-(e) y de las señales PPG (f)-(j), adquiridas de cinco individuos elegidos aleatoriamente. . . . . . . . . . . . .

6.16 Tendencia de la entropía de permutación (permutEn), conforme se van acumulando bloques de datos de longitud 1.000, de las señales de referencia y de las señales PPG, adquiridas de cinco individuos elegidos aleatoriamente; aparecen resaltadas las señales PPG, de forma que se reconozca notoriamente su naturaleza, contenida en el rango comprendido entre la aperiodicidad y el carácter cuasiperiódico. . . .

6.17 Modelo de difusión, de acuerdo con sucesiones temporales de 15.000 puntos, para un valor de $c=2,5$, de las señales de referencia (a)-(e) y de las señales PPG (f)-(j), adquiridas de cinco individuos elegidos aleatoriamente. . . . . . . . . . . . . . .

6.18 Capacidad difusiva, de acuerdo con sucesiones temporales de 15.000 puntos, de las señales de referencia (a)-(e) y de las señales PPG (f)-(j), adquiridas de cinco individuos elegidos aleatoriamente. En (d)-(e) las curvas de $M_{c}(n)$ y $D_{c}(n)$ no se aprecian pues al coincidir sus valores figuran superpuestas. . . . . . . . . . . . . . .

6.19 Coeficiente de difusión, de acuerdo con sucesiones temporales de 15.000 puntos, para 100 valores de $c$ elegidos al azar, de las señales de referencia (a)-(e) y de las señales PPG (f)-(j), adquiridas de cinco individuos elegidos aleatoriamente. 


\section{Índice de tablas}

2.1 Breve esquema de la historia de los sistemas dinámicos. . . . . . . . 33

2.1 Breve esquema de la historia de los sistemas dinámicos (cont.). . . . 34

4.1 Comportamientos dinámicos observados. . . . . . . . . . . . . . 86

4.2 Taxonomía de las dimensiones. . . . . . . . . . . . . . . . 116

5.1 Formas de energía biológicas y variables de medida. . . . . . . . . 155

6.1 Momentos estadísticos de las señales PPG. . . . . . . . . . . . . . . . 197

6.2 Parámetros $\tau$ y $m$ de las señales de referencia y de las señales PPG, adquiridas de cinco individuos elegidos aleatoriamente. . . . . . . . 202

6.3 Mayor exponente de Lyapunov $\lambda_{\text {máx }}$ con contraste de hipótesis para un nivel de significación del $5 \%$. La hipótesis nula $\mathbf{H}_{0}: \lambda_{\text {máx }} \geqslant 0$ indica la presencia de un comportamiento caótico. La hipótesis alternativa $\mathbf{H}_{1}: \lambda_{\text {máx }}<0$ sugiere una dinámica no caótica. $\mathrm{Si} \mathbf{H}=0$ no se rechaza la hipótesis nula; en caso contrario, si $\mathbf{H}=1$ se rechaza la hipótesis nula. En todos los casos se identifica el intervalo de confianza (IC). Presumiblemente, un intervalo de confianza infinito es consecuencia del tamaño limitado de la sucesión temporal, al igual que también podría derivarse de la dispersión de los datos; a mayor dispersión de los datos la conclusión es menos cierta y el intervalo de confianza es mayor. . . . . . . . . . . . . . . . . .

6.4 Espectro de exponentes de Lyapunov, conforme al método de M. Sano et al., así como la dimensión de Lyapunov, o dimensión de KaplanYorke $D_{\mathrm{KY}}$, y la entropía de Kolmogórov-Sinái $h_{\mathrm{KS}}$, de acuerdo con los postulados de una aproximación preliminar de la identidad de Pesin.205

6.4 Espectro de exponentes de Lyapunov, conforme al método de M. Sano et al., así como la dimensión de Lyapunov, o dimensión de KaplanYorke $D_{\mathrm{KY}}$, y la entropía de Kolmogórov-Sinái $h_{\mathrm{KS}}$, de acuerdo con los postulados de una aproximación preliminar de la identidad de Pesin (cont.). . . . . . . . . . . . . . . . . 206 
6.5 Dimensión fractal $D_{F}$ de cinco señales PPG de cinco individuos diferentes. Con objeto de contrastar la validez de los métodos se han generado señales de dimensión fractal conocida, esto es, la función coseno de Weierstrass (WCF), de $D_{F}=1,2$, la función coseno de Weierstrass-Mandelbrot (WMCF), de $D_{F}=1,3$, la función de Takagi (TF), de $D_{F}=1,5$, y un movimiento browniano fraccionario (fBm), de $D_{F}=1,7$. Las técnicas empleadas atienden al método de Higuchi, al método de Katz, al método de Sevcik, al método de conteo de cajas de resolución múltiple, o Multiresolution Box-counting Method (MRBC), al método de conteo de cajas de resolución de longitud múltiple, o Multiresolution Length Box-counting Method (MRLBC), y al método de retornos logarítmicos, o Delayed log-returns. . . . . . . . .

6.6 Dimensiones de información $\left(D_{1}\right)$ y de correlación $\left(D_{2}\right)$ de las señales de referencia y de las señales PPG, adquiridas de cinco individuos elegidos aleatoriamente. . . . . . . . . . . . . .

6.7 Entropía de dispersión (dispEn). Sobre una sucesión temporal de 15.000 puntos se calcula la entropía de dispersión de bloques de datos de 1.500 puntos con un $80 \%$ de solapamiento entre los sucesivos bloques, procediéndose seguidamente a desvelar su media y desviación

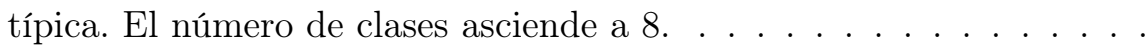

6.8 Entropía aproximada (ApEn). Sobre una sucesión temporal de 15.000 puntos se calcula la entropía aproximada de bloques de datos de 1.500 puntos con un $80 \%$ de solapamiento entre los sucesivos bloques, procediéndose seguidamente a desvelar su media y desviación típica. El valor de $r$ asciende a un $20 \%$ de la desviación típica de la sucesión temporal estandarizada. En la tabla se muestra la desviación típica $\sigma$ de la sucesión temporal normalizada. . . . . . . . . . . . . . .

6.9 Entropía de muestra multiescala (multescEn). Sobre una sucesión temporal de 15.000 puntos se calcula la entropía de muestra para 25 factores de escala, procediéndose seguidamente a desvelar su media y desviación típica. El valor de $r$ asciende a un $20 \%$ de la desviación típica de la sucesión temporal estandarizada. . . . . . . . . . . .

6.10 Entropía de permutación (permutEn). Sobre una sucesión temporal de 15.000 puntos se calcula la entropía de permutación de ventanas temporales de 250 puntos, procediéndose seguidamente a desvelar su media y desviación típica. El orden de los patrones ordinales asciende a 3. . . . . . . . . . . . . . . . . . . . 218

6.11 Complejidad de Lempel-Ziv para las diferentes señales de referencia. 224 


\section{Capítulo 1}

\section{Introducción}

La fuente primera de la ciencia no es, pues, el razonamiento, sino la comprobación detallada de una asociación propuesta por la imaginación.

- Paul Claudel, Diario

El empeño por comprender los sistemas fisiológicos, en los que infinidad de subsistemas operan conjunta y coordinadamente, y a diferentes escalas espacio-temporales, ha sido una constante desde tiempos remotos. No obstante, no fue hasta la era tecnológica cuando estos sistemas comenzaron a atraer la atención de otras disciplinas científicas que no fueran las estrictamente médicas. El equipamiento clínico con el que cuentan los hospitales modernos ha experimentado un desarrollo extraordinario. El instrumental con el que pueden adquirirse hoy día señales biológicas está al alcance de cualquiera y, por consiguiente, la disponibilidad de bases de datos con ingentes cantidades de medidas fisiológicas está creciendo de forma inimaginable. Si a esta eventualidad se suma la capacidad de cómputo actual, con visos de aumentar exponencialmente en las próximas décadas, el caldo de cultivo para un examen en profundidad de señales biológicas, con técnicas de análisis cada vez más sofisticadas, está servido. En este sentido cabe remarcar que el presente estudio investigativo nace, en el marco del proyecto ES3: intentar cuantificar y medir el nivel de estrés (véase el apéndice A para más referencias), con el propósito de caracterizar dinámicamente la señal PPG, acrónimo de PhotoPlethysmoGraphic, de forma que, a modo de fase previa, se adopten pasos firmes en la dirección que apunte a una mejor comprensión de los mecanismos que intervienen en el acoplamiento mutuo entre los subsistemas cardiovascular y respiratorio, enseñas de la información contenida en un ciclo característico de la señal PPG. El hecho de poder descifrar la dinámica connatural al proceso fisiológico imbricado en un ciclo PPG no solo posibilitará la definición de un modelo matemático con el que poder experimentar diferentes escenarios biológicos, sino que también aumentarían las expectativas de poder determinar indicadores diagnósticos que identifiquen cuándo un individuo está sometido a una situación de estrés. Que este trabajo se concentre en la señal PPG y no en otras 
señales biológicas, igualmente adquiridas en el proyecto ES3 y que se prestarían a idéntica metodología de análisis, obedece en esencia a la relativa carencia de estudios que, sobre la base de la línea argumental que aquí se propone, reparan en la señal PPG.

La figura 1.1 esquematiza la estructura que sugiere este estudio y que permite de un vistazo abrazar la guía metodológica desplegada en este memorándum. Más abajo se detalla someramente el contenido de cada capítulo.

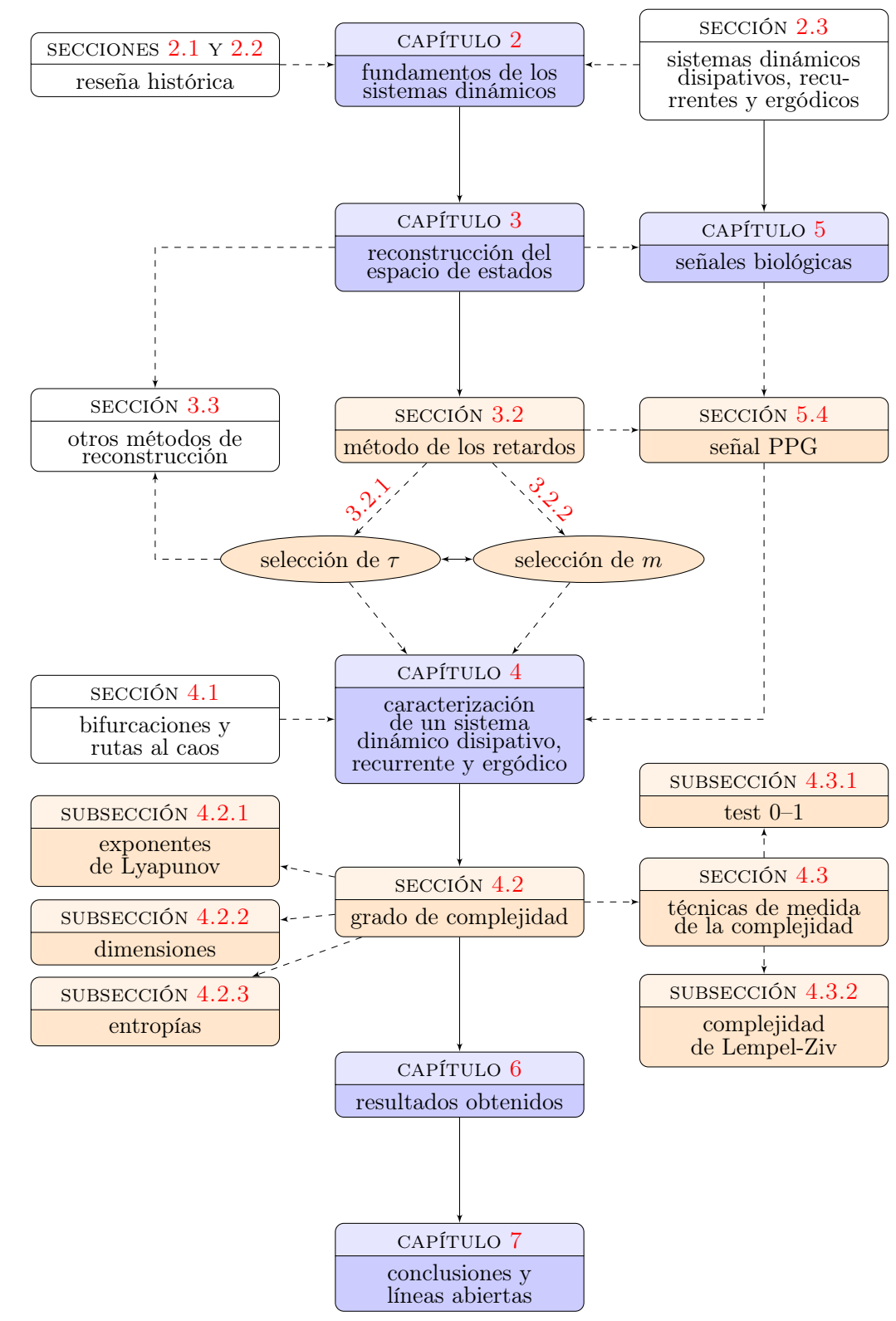

Figura 1.1: Diagrama de flujo de las dependencias conceptuales entre los capítulos.

En el capítulo 2 se justifica cronológicamente cómo los sistemas dinámicos no lineales hicieron acto de aparición al tiempo que el aparato matemático para su des- 
cripción y análisis, con el soporte ubicuo e inestimable de la computación. Henri Poincaré intuyó cualitativamente, a finales del siglo XIX, la presencia de comportamientos erráticos en el ámbito de la mecánica celeste a partir de un enfoque geométrico y topológico. Sin saberlo, había colocado la primera piedra de lo que hoy en día se conoce como la teoría del caos. A este hito histórico ha de añadírsele la contribución de Edward Lorenz en 1963 con su modelo metereológico simulado en un ordenador, en el que se constató la naturaleza impredecible de los modelos de comportamiento de ciertos sistemas dinámicos, los modelos caóticos. Desde entonces, si bien es probable que quede espacio para nuevos hallazgos, muchos otros nombres propios han enriquecido la teoría del caos - con innumerables aportaciones provenientes de diferentes ramas del conocimiento-, destapando toda una gama singular de comportamientos dinámicos y las posibles transiciones entre ellos, según se ha venido observando experimentalmente. En el contexto de las señales biológicas, objeto de este trabajo, se considera que los sistemas fisiológicos de los que se adquieren son disipativos, recurrentes y ergódicos, esto es, sistemas disipativos en cuanto a la disipación energética, dado que esta resulta crucial en la dinámica biológica; sistemas recurrentes y ergódicos en lo que respecta a la naturaleza cíclica de la dinámica de los procesos fisiológicos, lo que faculta la determinación de la distribución probabilística de los diferentes estados del sistema a partir de una simple sucesión temporal, sin necesidad de requerir todo un ensamble de mediciones. Sin embargo, lo más frecuente es disponer únicamente de un observable o sucesión temporal escalar; se carece de los estados posibles y de las ecuaciones que gobiernan la evolución temporal del comportamiento dinámico del proceso fisiológico, por lo que ha de recurrirse a los teoremas de Takens y de Birkhoff a fin de reconstruir la dinámica original del sistema fisiológico subyacente a partir de una sucesión temporal observada, garantizando simultáneamente que se conserven las propiedades de la dinámica original.

En el capítulo 3 se pormenoriza la fundamentación matemática que legitima la reconstrucción del espacio de estados, de forma que a partir de una sucesión temporal univariable sea posible inferir muchas propiedades invariantes del sistema dinámico del que ha sido medida. Dado que es virtualmente imposible obtener un espacio de reconstrucción exactamente igual que el espacio de estados originario, al menos sí es posible establecer un espacio de reconstrucción cuyas propiedades sean equivalentes a las que ostenta el espacio de estados natural. Un aspecto clave del proceso de reconstrucción conlleva una acertada elección de los parámetros $\tau$ y $m$, a saber, del retardo entre las diferentes componentes del vector de estado reconstruido y del número de componentes del mismo, respectivamente. En cierta medida cada vector de estado reconstruido debe contener información de la interacción mutua que coexiste entre las variables dinámicas originales que conforman los estados. En un escenario real, con ruido acoplado en los datos, la incertidumbre siempre está presente, de ahí que de la correcta designación de $\tau$ y $m$ dependa que el modelo dinámico reproduzca fielmente la dinámica del comportamiento del sistema físico. En cualquier caso los diferentes métodos, como el método de los retardos, comportan una primera aproximación a un modelo dinámico de un sistema físico del que únicamente se conoce un observable y el tiempo entre muestras.

En el capítulo 4 se sientan las bases de la conceptualización matemática del comportamiento dinámico de un sistema disipativo, recurrente y ergódico, a fin de justi- 
ficar cómo a partir de una sucesión temporal escalar, adquirida experimentalmente, es viable obtener información dinámica del proceso físico subyacente sobre la base de una reconstrucción del espacio de estados. Salvando las distancias, las señales PPG debieran esconder dinámicas similares a las señales de referencia, de ahí que, antes de su corroboración, se introduzcan los principios físicos en los que se fundamentan los comportamientos dinámicos observados por el momento. La prodigalidad funcional que encierran los sistemas caóticos, y que podría extenderse fácilmente a los sistemas fisiológicos, en los que la versatilidad adaptativa es sinónimo de supervivencia en un entorno circundante tan cambiante, testimonia el estudio de la teoría del caos y las transiciones hacia este tipo de dinámicas, sin eludir que el ruido puede llevar en muchos casos a juicios precipitados y no siempre acertados. Desde esta perspectiva se especifican los elementos que configuran la complejidad dinámica: los exponentes de Lyapunov, las dimensiones y la entropía. Puesto que el punto de partida es una sucesión temporal escalar diversos algoritmos se proponen con objeto de evaluar los ingredientes de la complejidad y así poder establecer equiparaciones entre los diferentes comportamientos dinámicos presentes en los sistemas físicos de interés. Para completar el armazón analítico se presentan dos técnicas de medida de la complejidad complementarias: el test $0-1$ y la complejidad de Lempel-Ziv. La primera de ellas aborda la complejidad en relación con el comportamiento difusivo de una respuesta temporal a medida que se despliega su dinámica en el espacio; en el segundo caso se recurre a la dinámica simbólica para evaluar la complejidad algorítmica de una sucesión temporal como si de una fuente de información se tratara.

El capítulo 5 arranca con una reseña histórica de las investigaciones fisiológicas a nivel experimental en la que se aprecia claramente el carácter multidisciplinario de los estudios fisiológicos y cómo la ingeniería, con el avance tecnológico sin precedentes de los últimos años, ha irrumpido en la biomedicina aportando las herramientas necesarias para capturar y almacenar digitalmente las señales biológicas. Una vez almacenados los datos fisiológicos en un ordenador, se está en disposición de poder acometer evaluaciones diagnósticas de acuerdo con la información contenida en los mismos. En este sentido ha de enfatizarse la dificultad que comporta el análisis de señales biológicas, consecuencia del hecho de que las señales capturadas proceden de sistemas abiertos y, por consiguiente, su verdadera naturaleza, ya de por sí degradada por efecto del ruido observacional introducido por el propio sistema de adquisición, puede quedar enmascarada de resultas de fenómenos adversos como la ausencia de estacionariedad o la presencia de ruido dinámico. Teniendo en cuenta que este estudio investigativo dirige sus esfuerzos hacia la caracterización dinámica de la señal PPG, el capítulo concluye con una descripción de la forma de onda de la señal PPG, cómo se procede habitualmente con su captura electrónica y las aplicaciones hasta ahora refrendadas o en vías de desarrollo.

En el capítulo 6 se presentan los resultados obtenidos cuando las diferentes técnicas de análisis, detalladas y argumentadas teóricamente en los capítulos 3 y 4, se aplican tanto a las señales PPG como a las señales de referencia, cuyo comportamiento es conocido a priori. De las características dinámicas conjuntas de las señales PPG, en comparación con las de las señales de referencia, se extraen, para cada método, las disquisiciones oportunas.

En el capítulo 7 se evidencian sintéticamente las conclusiones derivadas del aná- 
lisis y la interpretación de los resultados recogidos en el capítulo 6. A la luz de los mismos se observa claramente cómo las señales PPG no exhiben en principio un comportamiento caótico o aleatorio, sino más bien una respuesta que combina de una manera un tanto inextricable las dinámicas aperiódica y cuasiperiódica; eso sí, a bajas frecuencias o menor resolución temporal, el comportamiento de la señales PPG sugiere una marcada tendencia hacia comportamientos caóticos o aleatorios. Esta inclinación podría revelar bien una transición al caos o bien una configuración dinámica fundamentada en los atractores extraños no caóticos. Asimismo, en este capítulo se enumeran las líneas de investigación que se proponen tras la consecución de este trabajo inaugural. Los diferentes itinerarios abordan genéricamente las fases de modelización (diseño), análisis, simulación y control, si acaso, desde varias perspectivas metodológicas. Dado que una de las aspiraciones futuras de este estudio es determinar cualitativa y cuantitativamente, en el marco de un proyecto de mayor envergadura, cuándo un individuo está estresado y cómo los procesos fisiológicos involucrados reaccionan a fin de restablecer el equilibrio fisiológico o, en otro extremo más crítico, identificar la presencia de patologías más graves. Sobre la base de una concepción no lineal de los procesos fisiológicos que subyacen a las señales biológicas, una descripción geométrica, e inclusive estocástica, de las señales PPG, aparte de otras consideraciones, como la exploración de su comportamiento histerético, podría desentrañar aspectos funcionales del acoplamiento mutuo entre los subsistemas fisiológicos cuando un individuo es sometido a una situación de estrés o en presencia de alguna irregularidad orgánica.

Finalmente, en sendos apéndices auxiliares se describen, por un lado, las señales de referencia y la procedencia de las señales PPG empleadas en este estudio, amén de las herramientas software y hardware que han servido de soporte para la consecución del mismo, y, por otro lado, se refieren las publicaciones que hasta la fecha sostienen la calidad del trabajo de investigación desarrollado. 



\section{Capítulo 2}

\section{Sistemas dinámicos}

En este capítulo se introduce inicialmente, en las secciones 2.1 y 2.2, una pequeña reseña histórica de los orígenes y el devenir de los sistemas dinámicos, en general, y de los sistemas caóticos, en particular, para, acto seguido, en la sección 2.3, afrontar más formalmente aquellas definiciones y teoremas, en relación con los sistemas dinámicos, que justificarán posteriores desarrollos conceptuales.

\subsection{Breve panorámica histórica}

A lo largo de los siglos el ser humano se ha sentido atraído por el mundo que le rodea, como ya apuntó con brillantez el genio matemático francés Henri Poincaré (1854-1912): «Los científicos no estudian la naturaleza porque sea útil; la estudian porque les place, y les place porque es bella. Si la naturaleza no fuese bella, no valdría la pena conocerla, no valdría la pena vivir la vida» (Henri Poincaré [Poincaré, 2013]).

Las diferentes líneas de pensamiento científico no han sido pocas, si bien muchas de ellas derivaron, en la mitad del siglo XX, hacia una corriente estructuralista en la que, por un lado, el centro de atención se situaba en la fundamentación axiomática, los primeros principios, de las leyes que gobiernan los fenómenos físicos observados, y, por otro lado, sobre la base de la extensa producción matemática, y su tendencia a la abstracción, fue impregnándose de aspectos fenomenológicos, que irrumpieron con fuerza en el análisis de los procesos físicos, proporcionando todo un aparato matemático y físico al respecto.

Sin lugar a dudas, una de las áreas protagonistas de la revolución científica del último siglo fue el estudio del movimiento, la teoría de los sistemas dinámicos. Los avances en esta área, cuyos orígenes se remontan a la teoría de las ecuaciones diferenciales, promovida por Isaac Newton (1643-1727) y Gottfried Leibniz (16461716), han permitido que hoy día comience a comprenderse la evolución dinámica de sistemas caóticos, a saber, sistemas que evolucionan de forma perpetua sin pauta aparente.

Las matemáticas han posibilitado capturar la esencia del movimiento de los astros e incluso predecirlo. No obstante, las descripciones matemáticas que tradicionalmente predecían fielmente la evolución de ciertos sistemas sometidos a leyes físicas o biológicas conocidas, por azarosas que se antojaran, no fueron capaces de capturar el 
complejo movimiento de la atmósfera, el flujo turbulento en la mecánica de fluidos, o, incluso, la dinámica inherente a cualquier sistema fisiológico. En este sentido la teoría del caos ha arrojado algo de luz desde la última mitad del siglo XX.

A finales del siglo XIX se levantaba un muro infranqueable entre la facilidad con la que era posible examinar los denominados sistemas dinámicos lineales y la dificultad que entrañaba el análisis de los llamados sistemas dinámicos no lineales. Los sistemas lineales se caracterizan por pautas de comportamiento sencillas, con transiciones hacia un estado de equilibrio (estado de reposo) o hacia un movimiento regular o periódico. Por el contrario, los sistemas no lineales eran calificados de intratables. El fracaso al que se enfrentaba todo análisis de estos sistemas no residía en la ausencia de habilidad científica, sino más bien en la óptica para abordar problemas de esta índole, pues en muchos casos se pretendió, sin demasiado éxito, resolver las ecuaciones diferenciales que formulaban el movimiento bajo estudio. No fue hasta la aparición de los ordenadores cuando pudo reconducirse la situación, dado que estos dispositivos electrónicos favorecieron la simulación del movimiento de los sistemas no lineales, evidenciándose dinámicas que diferían sustancialmente de la sencilla dinámica lineal, como la dinámica caótica, que, con el transcurso de los años, ha acreditado su omnipresencia en la naturaleza.

Con el tiempo se han ido diluyendo concepciones erróneas de los sistemas aparentemente complejos. En primer lugar las reglas que gobiernan la dinámica compleja de un sistema no siempre derivan, como se creía hasta entonces, de la interacción entre las muchas variables que pueden determinar el estado del sistema en un momento concreto. La complejidad puede originarse como consecuencia de la no linealidad consustancial al sistema, incluso en sistemas caracterizados por una única variable. En segundo lugar debe abandonarse todo intento estéril de resolución de las ecuaciones de movimiento no lineal, optando más bien por profundizar en la comprensión de las pautas «regulares» que pueden entreverse en su comportamiento, es decir, todo intento por conocer con exactitud la evolución futura de un sistema está condenado al fracaso, pero, de otro modo, es viable estimar su evolución probable. La teoría de la probabilidad constituye así un ingrediente esencial en el análisis de sistemas caóticos. En la misma línea la teroía ergódica prueba que es posible extraer las pautas de comportamiento de un sistema complejo por medio de su simple observación.

Una vez reconocidas la simulación por ordenador y la observación empírica como fuentes de conocimiento del comportamiento que rige la dinámica de los sistemas complejos, la barrera que delimita la física y las matemáticas de los sistemas dinámicos es cada vez más sutil. La teoría del caos ha experimentado un fuerte impulso durante los últimos cuarenta años, lo que ha permitido presentar los resultados conseguidos de una forma clara e intuitiva. Su fácil exposición y comprensión se deben, en parte, a que muchos modelos caóticos, no lineales por naturaleza, son extremadamente sencillos en su descripción; de ahí que la teoría caótica no sea una forma superior o más complicada de hacer matemáticas. Es una forma diferente de enfocar el análisis de sistemas con comportamientos aparentemente erráticos. 


\section{$2.2 \quad$ Historia de los sistemas dinámicos}

La dinámica originariamente fue una rama de la física, si bien con el tiempo ha adquirido un carácter marcadamente multidisciplinario. La raíz de la disciplina afloró a la superficie a mediados del siglo XVII, cuando Isaac Newton ideó las ecuaciones diferenciales, y descubrió las leyes del movimiento y de la gravitación universal, mecanismos con los que pudo explicar las leyes de Kepler sobre el movimiento de los planetas. Particularmente, Newton resolvió el problema de los dos cuerpos, esto es, calcular el movimiento de la Tierra alrededor del Sol, dada la atracción gravitacional entre ellos. Generaciones posteriores de físicos y matemáticos pretendieron, sin éxito, extender el método analítico de Newton al problema de los tres cuerpos celestes, la Tierra, la Luna, y el Sol. La imposibilidad de definir explícitamente las ecuaciones de movimiento de los tres planetas fue el denominador común de denodados esfuerzos durante décadas.

El punto de inflexión vino de la mano de los trabajos emprendidos por Henri Poincaré, quien, a finales del siglo XIX, introdujo una perspectiva que reparaba más en los aspectos cualitativos del problema de los tres cuerpos que en los detalles cuantitativos. Por ejemplo, en vez de plantearse la posición exacta de los planetas en un instante concreto, puso en entredicho la estabilidad del sistema solar, valorando la posibilidad de que algunos planetas pudieran alejarse hacia los confines del universo. Para dar cumplida respuesta a estas inquietudes, Poincaré desarrolló una formidable aproximación geométrica y topológica que le facilitara el análisis de los interrogantes relacionados con la resolución, en definitiva, de ecuaciones diferenciales no lineales. La fundamentación geométrica y topológica es tan sólida que ha impregnado la teoría de los sistemas dinámicos en su conjunto, con aplicaciones que se extienden más allá de la mecánica celeste. Poincaré fue el primero en vislumbrar la presencia del caos, por el que un proceso determinista muestra un comportamiento aperiódico muy dependiente de las condiciones iniciales, imposibilitándose así la predictibilidad a largo plazo.

Durante la primera mitad del siglo XX el caos permaneció en el baúl de los recuerdos. ${ }^{1}$ En el transcurso de esos años la teoría de los sistemas dinámicos se ocupó principalmente de las oscilaciones no lineales y su aplicabilidad en los ámbitos de la física y de la ingeniería. Los osciladores no lineales desempeñaron un papel tras-

\footnotetext{
${ }^{1}$ Durante mucho tiempo el estudio de los sistemas dinámicos quedó relegado a un segundo plano, debido, principalmente, a que los matemáticos se sintieron más atraídos por las estructuras geométricas escondidas en las soluciones de los sistemas dinámicos. Las variedades, los espacios naturales en que habitan los sistemas dinámicos, se convirtieron en objeto de estudio por derecho propio [Lee, 2010]. Materias como la geometría diferencial [Lee, 2013], la topología diferencial [Hirsch, 2012] y la topología algebraica [Bredon, 2013] introdujeron técnicas muy valiosas para abordar problemas geométricos desde diferentes perspectivas. En los años 60, y de forma independiente, Jürgen Moser y Stephen Smale, en EE. UU., Maurício Peixoto, en Brasil, y Andréi Kolmogórov, Vladimir Arnold y Yákov Sinái, en la antigua Unión Soviética, idearon métodos, conforme a la topología diferencial, que permitieron analizar el comportamiento caótico de ciertos sistemas dinámicos, conocidos como sistemas hiperbólicos o de axioma A [Barreira, 2012, Ruelle, 1989]. La conjunción de geometría y análisis matemático, junto con la posterior contribución de la teoría KAM, promovió el desarrollo de diversas ramas del saber, como la teoría ergódica diferenciable [Barreira and Pesin, 2013], la dinámica topológica [Akin, 2010], la mecánica hamiltoniana [Seimenis, 2013], o la teoría cualitativa de ecuaciones diferenciales ordinarias [Smale et al., 2003].
} 
cendental en el desarrollo de tecnologías punteras, como la radio, el radar o el láser. En términos teóricos los osciladores no lineales promovieron nuevos útiles matemáticos; entre los pioneros de este nuevo bagaje matemático destacan las figuras de Balthasar van der Pol, Aleksandr Aleksandrovich Andronov, Aleksandr Mijáilovich Liapunov, Lev Semiónovich Pontriaguin, John Edensor Littlewood, Edward Cartwright, Norman Levinson, Stephen Smale y John Milnor, entre otros. Por otro lado, los métodos geométricos y topológicos de Poincaré experimentaron un fuerte impulso que derivó en una mejor comprensión de la mecánica celeste, gracias, fundamentalmente, a los trabajos de George David Birkhoff, que impulsó la dinámica discreta para una mejor comprensión del comportamiento de las ecuaciones diferenciales, y de Andréi Nikoláyevich Kolmogórov, Vladimir Igorevich Arnold y Jürgen Kurt Moser, precursores de la teoría KAM, relacionada con la persistencia de movimientos cuasiperiódicos [Strogatz, 1994, Devaney, 2018].

La aparición del ordenador personal en los años 50 significó un cambio de rumbo en la trayectoria de la teoría de los sistemas dinámicos. La simulación de las ecuaciones matemáticas en un ordenador contribuyó a desarrollar y perfeccionar la intuición acerca de los comportamientos no lineales de las posibles soluciones de los sistemas dinámicos. De esta manera, experimentando con las ecuaciones en un ordenador, Edward Norton Lorenz descubrió en 1963 el movimiento caótico en un atractor extraño [Lorenz, 1963]. Por aquel entonces E. Lorenz estaba estudiando un modelo simplificado de convección atmosférica, con objeto de comprender mejor por qué es extremadamente complicado predecir el tiempo meteorológico. E. Lorenz advirtió que las soluciones de su modelo nunca alcanzaban un estado de equilibrio o un estado periódico; en su lugar las soluciones oscilaban de un modo un tanto irregular (aperiódico). Además, si se iniciaban las simulaciones a partir de dos condiciones iniciales ligeramente distintas, el comportamiento de las soluciones difería significativamente transcurrido un tiempo. La interpretación de estos resultados le condujo a considerar seriamente la naturaleza impredecible del modelo. Pequeños errores en la medición del estado actual de la atmósfera se amplificarían rápidamente, dificultándose en extremo cualquier pronóstico fiable. No obstante, E. Lorenz también probó la existencia de cierta estructura dentro del caos; si se representan las soluciones de su modelo en un espacio tridimensional, los puntos se distribuyen en lo que parecen ser las alas de una mariposa. El científico atribuyó a este subconjunto de puntos el calificativo de «complejo infinito de superficies». Actualmente, este subconjunto de puntos conformaría un ejemplo de lo que se denomina un fractal.

Los trabajos de E. Lorenz tuvieron inicialmente escasa relevancia en la comunidad científica, hasta que en los años 70 se reavivó con fuerza el interés por el caos. En 1971 David Ruelle y Floris Takens propusieron una nueva teoría acerca de los mecanismos que intervienen en el flujo turbulento, sobre la base de la dinámica presente en los atractores extraños [Ruelle and Takens, 1971]. Unos años más tarde, Robert May encontró ejemplos de caos en mapas iterados, aplicados en el terreno de la población biológica. Robert May escribió un artículo muy influyente [May, 1976], destacando la importancia pedagógica de estudiar modelos no lineales muy simples, con el firme propósito de disuadir la concepción lineal tradicional de la naturaleza, vigente hasta entonces en muchos centros educativos. Posteriormente, Mitchell J. Feigenbaum efectuó uno de los descubrimientos más extraordinarios de este campo, la 
existencia de leyes universales que gobiernan la transición entre un comportamiento regular y uno caótico [Feigenbaum, 1983]; en otras palabras, sistemas completamente diferentes transitan hacia el caos de la misma manera. Su investigación permitió establecer un claro vínculo entre el caos y las transiciones de fase, lo que animó a muchos investigadores a profundizar en la teoría de los sistemas dinámicos. Finalmente, desde una óptica experimental sobresalen los trabajos relacionados con el comportamiento caótico de Jerry Gollub, en dinámica de fluidos, de Albert J. Libchaber, en las fluctuaciones convectivas de los sistemas Rayleigh-Benard, de Harry L. Swinney, en reacciones químicas, de Paul S. Linsay, en osciladores electrónicos, de Francis C. Moon, en sistemas mecánicos, y de Robert Westervelt, en semiconductores, entre otros [Baker and Gollub, 1996].

Si bien el caos se convirtió en el actor protagonista durante el boom de los años 70, conviene no olvidar otros papeles secundarios de especial importancia. Benoît Mandelbrot popularizó los fractales, creó muchos de ellos por ordenador y probó su aplicación a otros muchos campos del conocimiento [Mandelbrot, 1983, Peitgen et al., 2013, Falconer, 2013, Barnsley and Barnsley, 2006]. Por otro lado, Arthur Winfree destacó en el campo de la biología matemática, donde aplicó métodos geométricos de la teoría de los sistemas dinámicos a las fluctuaciones biológicas, como los ritmos circadianos o los ritmos cardíacos [Winfree, 1980].

Tabla 2.1: Breve esquema de la historia de los sistemas dinámicos.

\begin{tabular}{|c|c|c|}
\hline 1666 & Isaac Newton & $\begin{array}{l}\text { nace el cálculo; interpretación del mo- } \\
\text { vimiento planetario }\end{array}$ \\
\hline siglo XVIII & & $\begin{array}{l}\text { auge del cálculo y de la mecánica clá- } \\
\text { sica }\end{array}$ \\
\hline siglo XIX & & $\begin{array}{l}\text { estudios analíticos del movimiento de } \\
\text { los planetas }\end{array}$ \\
\hline década de 1890 & Henri Poincaré & $\begin{array}{l}\text { fundamentación geométrica y topo- } \\
\text { lógica; fracasos analíticos debidos al } \\
\text { desconocido caos }\end{array}$ \\
\hline $1920-1950$ & & $\begin{array}{l}\text { osciladores no lineales en física e inge- } \\
\text { niería; invenciones de la radio, el ra- } \\
\text { dar y el láser }\end{array}$ \\
\hline $1920-1960$ & $\begin{array}{l}\text { G. D. Birkhoff } \\
\text { A. N. Kolmogórov } \\
\text { V. Í. Arnold } \\
\text { J. K. Moser }\end{array}$ & $\begin{array}{l}\text { comportamiento complejo en mecáni- } \\
\text { ca hamiltoniana }\end{array}$ \\
\hline 1963 & Edward N. Lorenz & $\begin{array}{l}\text { atractor extraño en un modelo con- } \\
\text { vectivo muy simple }\end{array}$ \\
\hline
\end{tabular}


Tabla 2.1: Breve esquema de la historia de los sistemas dinámicos (cont.).

\begin{tabular}{|ll|}
\hline década de los 70 & flujo turbulento y caos \\
F. Takens & \\
Robert M. May & caos en el mapa logístico \\
& $\begin{array}{l}\text { M. J. Feigenbaum } \\
\text { universalidad y renormalización; co- } \\
\text { nexión entre el caos y las transiciones } \\
\text { de fase }\end{array}$ \\
& $\begin{array}{l}\text { estudios experimentales del caos } \\
\text { Arthur T. Winfree }\end{array}$ \\
& osciladores no lineales en biología \\
Benoît Mandelbrot & fractales \\
& interés generalizado por el caos, los \\
década de los 80 & aplicaciones \\
&
\end{tabular}

Ya en los años 80 la colectividad de investigadores trabajando en la teoría de los sistemas dinámicos era muy numerosa, y desde entonces cada año nuevos miembros pasan a engrosar esta incontable lista, de ahí que resulte muy difícil condensar en una simple tabla toda la historia que hay detrás de esta disciplina. Aun así, la tabla 2.1 ilustra los hitos principales que han contribuido al desarrollo de una teoría no consolidada del todo, pero con un futuro muy prometedor [Strogatz, 1994].

\subsection{Sistemas dinámicos disipativos, recurrentes y ergódi- $\cos$}

La voz dinámica sugiere fuerza, energía, movimiento o cambio. Un sistema dinámico determinista comprende algo que se mueve, cambia o evoluciona con el tiempo, siguiendo unas reglas que determinan indiscutiblemente sus valores futuros. Dentro de la rica variedad de comportamientos dinámicos, en el marco de la teoría de los sistemas dinámicos (el estudio de fenómenos que varían con el tiempo) no lineales (el estudio del movimiento o evolución no lineal), el caos ocupa un lugar preponderante como paradigma de lo que se entiende por una dinámica compleja determinista.

Los sistemas dinámicos suelen dividirse en dos grandes categorías genéricas. Los sistemas que convierten la energía mecánica en calor reciben la denominación de sistemas disipativos; en cambio aquellos sistemas que preservan la energía mecánica se llaman conservativos [Sprott, 2003]. En los sistemas conservativos, regidos por una evolución temporal hamiltoniana, se conserva todo volumen en el espacio de estados, 
conforme al teorema de Liouville, no perdiéndose en consecuencia energía [Almeida, 1990]. Por el contrario, en los sistemas disipativos, dado que se manifiesta fricción en el sistema, y, por consiguiente, pérdida de energía, un elemento de volumen se contrae a lo largo de una trayectoria en el espacio de estados, aproximándose con el tiempo a alguna condición asintótica o atractor, donde, por ejemplo, pudiera desarrollarse un comportamiento caótico. Un atractor es la región del espacio de estados, ya sea un único punto o un subconjunto de puntos, que, en el límite asintótico (infinitas iteraciones del operador de evolución del sistema), atrae todas las trayectorias que emanan de algún subconjunto de condiciones iniciales, denominado cuenca de atracción. El atractor comporta un subconjunto invariante, obligando a la dinámica del sistema a permanecer confinada en la región del espacio de estados delimitada por el propio atractor una vez el sistema transite por él.

En sentido estricto únicamente se observan atractores en los sistemas disipativos, si bien para que que un sistema dinámico disipativo continúe variando u oscilando con el tiempo se requiere una aportación energética externa, bien explícitamente por medio de un agente inductor no autónomo (dependiente del tiempo) o bien implícitamente mediante un mecanismo autónomo (independiente del tiempo) intrínseco que empuja al sistema fuera del equilibrio. En cualquier caso un consumo constante de energía es el factor determinante para que el sistema se mantenga fuera de una situación de equilibrio permanente. Los sistemas fisiológicos se circunscriben al ámbito de los sistemas dinámicos disipativos, dado que la disipación de energía resulta extremadamente útil para estabilizar la dinámica biológica y preservar el buen funcionamiento, en condiciones óptimas, de un organismo vivo [Brogliato et al., 2007, Seely and Macklem, 2012]; de ahí que este trabajo, referido, en particular, a la señal PPG, se limite al estudio de los sistemas dinámicos disipativos.

En el contexto de los sistemas dinámicos disipativos las propiedades de recurrencia y ergodicidad facilitan el análisis de los procesos físicos a partir de una sola sucesión temporal, que no es otra cosa que una sucesión o secuencia de mediciones de un observable, del todo habitual, o de varios observables de un sistema físico, realizadas a intervalos regulares de tiempo. ${ }^{2} \mathrm{El}$ principio básico que subyace al fenómeno de la recurrencia, planteado por Henri Poincaré en 1890 para estudiar el movimiento planetario, es que la trayectoria típica de un sistema en el que la dinámica se desarrolla en un volumen finito, véase un atractor, vuelve infinitamente a menudo a cualquier vecindad de su punto inicial. En lo que respecta a la ergodicidad, se satisface que, para la descripción estadística de un sistema dinámico, los promedios temporales de la evolución de una trayectoria típica del sistema coinciden con los promedios espaciales de las evoluciones de un ensamble de trayectorias.

En un sistema dinámico se dispone de un espacio de estados, en el que los puntos representan estados del sistema, y de una transformación u operador de evolución que se aplica reiteradamente a cada punto del espacio. Si el espacio de estados es un espacio de medida (noción de «volumen» pero no necesariamente en un espacio tridimiensional) y todo el espacio presenta una medida finita, se dice que la transformación preserva la medida si al aplicar la transformación a todos los puntos de un subconjunto del espacio, el subconjunto imagen tiene la misma medida que el

\footnotetext{
${ }^{2}$ Un observable es todo aquello que puede medirse; luego un estado del sistema dinámico queda definido completamente por el valor de todos los observables.
} 
subconjunto inicial. Esta consecuencia permite relacionar la recurrencia y la ergodicidad, si bien la recurrencia es más débil, menos restrictiva, que la ergodicidad, pues aquella no requiere que el sistema satisfaga la propiedad mezclante (mixing) y, por consiguiente, el espacio puede particionarse de forma que, para cada elemento de la partición, todo punto dentro del mismo tiende, con la transformación, a permanecer en él (recurrencia débil, o, con otras palabras, más local). En cambio, la ergodicidad impide la existencia de una partición dinámica, imponiéndose una recurrencia más generalizada en el espacio. Básicamente, la propiedad mezclante determina cierto grado de uniformidad dinámica, de modo que la fracción de tiempo que una trayectoria invierte en un subonjunto cualquiera $Y$ del espacio coincide con la fracción de la medida del espacio total ocupado por $Y$, esto es, la propiedad ergódica asegura que los promedios estadísticos de un ensamble de sucesiones temporales no cambian con el tiempo y, además, todos los estadísticos definidos, en el límite asintótico, para cada sucesión temporal son invariantes con independencia de la sucesión temporal, dentro del ensamble, sobre la que se evalúen. En este sentido resulta factible definir una medida natural (física), llamada en ocasiones medida invariante, densidad invariante o función de distribución de probabilidad continua, que describe con qué frecuencia son visitadas diferentes regiones del espacio por la trayectoria, dando pie a todo un repertorio de definiciones de dimensión que, en esencia, posibilitan determinar cuántas variables son necesarias para modelizar la dinámica de un sistema. Para más información sobre los conceptos revisados someramente en líneas precedentes se recomienda consultar [Eckmann and Ruelle, 1985, Farmer et al., 1983, Pawelzik and Schuster, 1987, Bai-lin, 1989, Hilborn, 2001, Kantz and Schreiber, 2003, Barreira and Pesin, 2013, Halmos, 2006, Ruelle, 1989].

\subsubsection{Sucesiones temporales y observables}

Un sistema dinámico queda caracterizado por el par $(\Omega, T)$, donde $\Omega$ identifica el espacio de estados que describe todos los posibles estados del sistema, y $T$ la dinámica que especifica cómo evoluciona temporalmente un punto $\omega \in \Omega$. La complejidad de un sistema dinámico se caracteriza por la impredecibilidad de su dinámica, esto es, cuanto más complejo es el sistema menos predecible es su comportamiento.

A fin de introducir una medida en el espacio de estados $\Omega$ de un sistema dinámico $(\Omega, T)$, se define una $\sigma$-álgebra $\mathcal{A}(\Omega)$, una familia de subconjuntos de $\Omega$ que satisfacen las siguientes condiciones:

(i) $\emptyset \in \mathcal{A}(\Omega)$,

(ii) si $A \in \mathcal{A}(\Omega)$, entonces $\Omega \backslash A \in \mathcal{A}(\Omega)$, y

(iii) si $A_{1}, A_{2}, \ldots, A_{n} \in \mathcal{A}(\Omega)$, entonces $\bigcup_{n=1}^{\infty} A_{n} \in \mathcal{A}(\Omega)$.

En el contexto de los sistemas dinámicos se asume que $\mu$ es una medida de probabilidad finita, es decir, $\mu(\Omega)=1$. La tripleta $(\Omega, \mathbb{B}(\Omega), \mu)$, siendo $\mathbb{B}(\Omega)$ una $\sigma$-álgebra de Borel, constituye un espacio de medida o, en el caso particular de que la medida $\mu$ sea una medida de probabilidad, un espacio de probabilidad.

Se dice que un mapa $T$ es una transformación que preserva la medida, en inglés designada $\mu$-preserving, si $\mu\left(T^{-1} B\right)=\mu(B)$, para todo $B \in \mathbb{B}(\Omega)$. Que la medida 
$\mu$ sea invariante, esto es, preservada por la función de transformación $T$, garantiza que la distribución de los estados del sistema no se modifica con la dinámica $T$. En la teoría caótica se suelen abordar sistemas dinámicos que preservan la medida $(\Omega, \mathbb{B}(\Omega), \mu, T)$, lo que significa que $\Omega$ es un espacio de estados, $\mathbb{B}(\Omega)$ es una $\sigma$-álgebra de Borel asociada a $\Omega, T: \Omega \hookrightarrow \Omega$ una transformación que preserva la medida, y $\mu: \mathbb{B}(\Omega) \rightarrow[0,1]$ una medida de probabilidad. La recurrencia de Poincaré es una propiedad esencial de todo sistema dinámico que preserva la medida, a saber, para cualquier subconjunto medible $B \in \mathbb{B}(\Omega)$, casi todo $\omega \in B$ regresa infinitamente a menudo a $B$ [Barreira, 2006, Barreira, 2008].

Dado un sistema dinámico $(\Omega, T)$, la dinámica $T$ construye, para cada estado inicial $\omega \in \Omega$, una órbita $\omega, T^{\circ 1} \omega, T^{\circ 2} \omega, \ldots, T^{\circ \infty} \omega$, donde $T^{\circ i}, i \in \mathbb{N}_{>0}$, identifica la $i$-ésima iteración de $\omega$. A nivel experimental se suele disponer únicamente de una sucesión temporal escalar, desconociéndose bien el espacio de estados del sistema dinámico o bien las ecuaciones que gobiernan su dinámica temporal. La relación que se establece entre el sistema dinámico y la sucesión temporal observada se designa como observable $X: \Omega \rightarrow \mathbb{R}$, una función de medición que asigna un valor real o escalar $\mathbb{R}$ a cada posible estado $\omega \in \Omega$. Desde una perspectiva matemática, un observable es un mapa medible de Borel. Dado un observable $X$ del sistema dinámico, se establece la sucesión temporal

$$
\left\{x_{i}\right\}_{i \in \mathbb{N}_{0}}=\left(X\left(T^{\circ i} \omega\right)\right)_{i \in \mathbb{N}_{0}},
$$

a partir de la órbita $\left(T^{\circ i} \omega\right)_{i \in \mathbb{N}_{0}}$. En ciertas ocasiones resulta más conveniente reunir más de un observable, p. ej., $N$ observables escalares que conforman el vector observable $\mathbf{X}=\left(X_{1}, X_{2}, \ldots, X_{N}\right)$, con objeto de recabar más información del sistema dinámico; son las denominadas sucesiones temporales multivariable, frente a las sucesiones temporales univariable, en las que solamente se dispone de un observable del sistema dinámico.

\subsubsection{Dinámica de un sistema a partir de una sucesión temporal}

En principio sería necesario disponer de todos los estados posibles y de las ecuaciones que rigen su evolución temporal para caracterizar completamente el sistema dinámico bajo estudio. No obstante, los teoremas de Birkhoff y de Takens posibilitan un análisis suplementario con una menor especificación del sistema dinámico. Concretamente, el teorema de Birkhoff permite extraer propiedades del sistema dinámico a partir de una única órbita para casi todo $\omega \in \Omega$, y el teorema de Takens posibilita la reconstrucción de la dinámica originaria $T$ a partir de una sucesión temporal observada.

\subsubsection{Teorema ergódico de Birkhoff}

Sea $T$ un mapa ergódico que permite la recuperación de aquellas propiedades relacionadas con un sistema dinámico que preserva la medida a partir de la órbita de un simple estado inicial $\omega$, para casi todo $\omega \in \Omega$.

Definición 2.1 Se dice que un mapa $T$ es ergódico si para todo $B \in \mathbb{B}(\Omega)$, con $T^{-1} B=B$, se cumple que $\mu(B)=0$ o $\mu(B)=1$. 
Un sistema dinámico $(\Omega, \mathbb{B}(\Omega), \mu, T)$ con una función de transformación $T$ ergódica recibe el nombre de sistema dinámico ergódico. En líneas generales un sistema dinámico ergódico no puede descomponerse en subsistemas que evolucionan de forma independiente, es decir, las órbitas de casi todos los estados iniciales $\omega \in \Omega$ evidencian aproximadamente las mismas propiedades de la dinámica $T$.

El teorema de Birkhoff establece uno de los resultados centrales de la teoría ergódica.

Teorema 2.2 (Teorema ergódico de Birkhoff [Choe, 2006, Barreira, 2012]). Dados un sistema dinámico que preserva la medida $(\Omega, \mathbb{B}(\Omega), \mu, T)$ y una función integrable $f$, existe una función $f^{*}$ tal que $\int_{\Omega}\left|f^{*}\right| \mathrm{d} \mu(\omega)<\infty, f^{*}(T \omega)=f^{*}(\omega)$, para todo $\omega \in \Omega$. Además, para casi todo $\omega \in \Omega$

$$
\lim _{n \rightarrow \infty} \frac{1}{n} \sum_{i=0}^{n-1} f\left(T^{\circ i} \omega\right)=f^{*}(\omega) .
$$

Por otro lado, si $T$ es ergódica, entonces $f^{*}$ es constante y para casi todo $\omega \in \Omega$ se sigue que

$$
\lim _{n \rightarrow \infty} \frac{1}{n} \sum_{i=0}^{n-1} f\left(T^{\circ i} \omega\right)=\int_{\Omega} f \mathrm{~d} \mu(\omega) .
$$

En general, de acuerdo con el teorema ergódico de Birkhoff, la ergodicidad proporciona, a partir de una única órbita y para casi todo estado inicial $\omega \in \Omega$, toda la información de la distribución de la medida $\mu$. Otra propiedad trascendental de los sistemas dinámicos, más fuerte que la ergodicidad, es su carácter mezclante ( $m i$ xing) [Halmos, 2006].

Definición 2.3 Se dice que $T$ es mezclante, o fuertemente mezclante, si para todo $A, B \in \mathbb{B}(\Omega)$,

$$
\lim _{n \rightarrow \infty} \mu\left(T^{\circ-n} A \cap B\right)=\mu(A) \mu(B) .
$$

Comúnmente, la cualidad mezclante de un sistema dinámico implica que las iteraciones $T^{\circ n} \omega$ se vuelven, con el tiempo, casi independientes del estado $\omega$. En cierto sentido, para todo $A, B \in \mathbb{B}(\Omega)$, las iteraciones $T^{\circ n} A$ extienden, conforme a la propiedad de separación, las diferentes órbitas de $\omega \in A$ por todo el atractor, de forma que $\mu\left(T^{\circ-n} A \triangle B\right)=0$.

\subsubsection{Teorema de reconstrucción de Floris Takens}

El teorema de Takens está íntimamente ligado a la reconstrucción del espacio de estados a partir de una sucesión temporal univariable. La idea básica consiste en sustituir la sucesión temporal originaria $\left\{x_{t}\right\}_{t \in \mathbb{N}_{0}}$ por una sucesión de vectores $\left(x_{t}, x_{t+1}, \ldots, x_{t+m-1}\right)_{t \in \mathbb{N}_{0}}$, de forma que estos preserven las propiedades del sistema dinámico subyacente. Aquella dimensión vectorial que contiene toda la dinámica 
del sistema subyacente recibe el nombre de dimensión de encaje (embedding dimension). Por el teorema de Takens, si $m$ es suficientemente grande resulta factible recuperar las propiedades de la dinámica natural a partir del comportamiento de los vectores reconstruidos [Borovkova, 1998].

Sea $(\Omega, T)$ un sistema dinámico con un espacio de estados $\Omega$ de dimensión finita y órbitas positivas acotadas $\left\{T^{\circ n} \omega\right\}_{n \in \mathbb{N}_{0}}$, para todo $\omega \in \Omega$. Una función de medición relaciona el sistema dinámico con la sucesión temporal $X: \Omega \rightarrow \mathbb{R}$. Normalmente, $X(\omega)$ mapea la medición de un estado $\omega \in \Omega$ a un número real $\mathbb{R}$. Si $\left(\omega_{0}, T^{\circ 1} \omega_{0}, T^{\circ 2} \omega_{0}, \ldots\right)$ describe una órbita del sistema dinámico para un estado inicial $\omega_{0}$, entonces la correspondiente sucesión temporal de la órbita se obtiene cuando se aplica $X$ a cada punto de la órbita, esto es, $\left(X\left(\omega_{0}\right), X\left(T^{\circ 1} \omega_{0}\right), X\left(T^{\circ 2} \omega_{0}\right), \ldots\right)$.

Sean $X, T \in C^{\infty}$ continuamente diferenciables, y $\operatorname{Rec}_{m}: \Omega \rightarrow \mathbb{R}^{m}$ el mapa de reconstrucción vectorial que atiende a

$$
\operatorname{Rec}_{m}=\left(X\left(\omega_{0}\right), X\left(T^{\circ 1} \omega_{0}\right), \ldots, X\left(T^{\circ m-1} \omega_{0}\right)\right) \in \mathbb{R}^{m}
$$

Teorema 2.4 (Teorema de reconstrucción de Takens [Takens, 1981]). En el producto cartesiano $T \times X$ existe un subconjunto $U$ abierto y denso, tal que si $(T, X) \in U$ el mapa de reconstrucción $\operatorname{Rec}_{m}$ es un encaje siempre que $m>2 \cdot \operatorname{dim}(\Omega)$.

El teorema 2.4 conlleva que la condición $(T, X) \in U$ es válida para mapas $T$ y observables $X$ genéricos, si bien el procedimiento de reconstrucción de un espacio de estados acarrea diversos problemas [Sauer et al., 1991]. Las condiciones impuestas por el teorema pretenden excluir casos excepcionales para los que la reconstrucción falla, como el escenario en que el observable $X$ sea una constante, o que el mapa $T$ sea la función identidad. En el primer caso mapear todos los puntos de una órbita a una constante destruye toda la información que contiene, desaprobando cualquier intento de reconstrucción; en el segundo caso, a partir del segundo punto de la órbita no se dispone de información adicional, por lo que la reconstrucción vuelve a manifestar su inoperancia.

En el proceso de encaje la transformación de $\Omega$ a $\mathbb{R}^{m}$, por medio del mapa de reconstrucción $\operatorname{Rec}_{m}$, conforma un encaje si el mapeo de $\Omega$ a $\mathbb{R}^{m}$ es continuamente diferenciable, al igual que su inversa. Esto significa que $\Omega$ y su imagen bajo el mapa de reconstrucción $\operatorname{Rec}_{m}$ se asocian mediante un difeomorfismo. Además, en presencia de un atractor $A \subset \Omega$, el mapa de reconstrucción transforma $A$ en su imagen $\mathbb{R}^{m}$ de forma que se preserve la estructura diferencial del atractor. De igual manera, la medida invariante del atractor reconstruido corresponde a la medida invariante de la transformación originaria. Por otra parte, dado que un difeomorfismo, restringido a un subconjunto acotado, introduce una «distorsión acotada» en las distancias entre puntos, la dimensión de correlación del atractor inicial y del atractor reconstruido es idéntica [Takens, 1981].

Para reconstruir un atractor a partir de una sucesión temporal escalar $\left\{s_{n}\right\}_{n \in \mathbb{N}_{0}}$, se determinan los vectores de reconstrucción conforme a

$$
\mathbf{x}_{n}=\operatorname{Rec}_{m}\left(T^{\circ n} \omega_{0}\right)=\left(s_{n}, s_{n+1}, \ldots, s_{n+m-1}\right),
$$


para $m \geqslant 2 \cdot \operatorname{dim}(\Omega)+1$. De esta forma, la trayectoria $\left\{T^{\circ n} \omega_{0}\right\}_{n \in \mathbb{N}_{0}}$ descrita en $\Omega$ es equivalente, debido a la acción del difeomorfismo, a la trayectoria descrita por los vectores de reconstrucción $\left\{\mathbf{x}_{n}\right\}_{n \in \mathbb{N}_{0}}$ en $\mathbb{R}^{m}$. En este sentido los vectores de reconstrucción se acumulan en una vecindad de $\mathbb{R}^{m}$, llamada subconjunto límite, que es difeomórfico al subconjunto hacia el que convergen la órbitas descritas por la acción de $T$ en $\Omega$. Así, la medida invariante de este subconjunto límite, al igual que la dimensión de correlación, o, inclusive, algunas otras peculiaridades inmanentes a la sucesión $\left\{\mathbf{x}_{n}\right\}_{n \in \mathbb{N}_{0}}$, caracterizan propiedades intrínsecas de la dinámica del sistema subyacente, pues no se ven condicionadas por la correspondiente función de medición u observable $X$.

Si se desconoce que la sucesión temporal ha sido generada por un sistema dinámico determinista, debido principalmente a una aportación significativa de ruido, la validez de los resultados de una reconstrucción suscita serias dudas. Aun así, un factor crítico del proceso de reconstrucción es la elección del valor de $m$, dado que es difícil seleccionar un valor de $m$ suficientemente grande cuando la dimensión de $\Omega$ se ignora, lo que impide aplicar directamente el criterio del teorema $2.4, m>2 \cdot \operatorname{dim}(\Omega)$. Si bien se han planteado diversos métodos para especular un valor apropiado de $m$ [Casdagli, 1992], todavía no se ha probado, salvo en el caso de los sistemas de axioma A [Ruelle, 1976, Ruelle, 1989], qué criterios determinan la adquisición de un proceso estocástico estacionario, ${ }^{3}$ respecto a alguna medida de probabilidad ergódica, a partir de una sucesión de vectores de reconstrucción. En cualquier caso, a efectos prácticos, suele presuponerse que la sucesión $\left\{\mathbf{x}_{n}\right\}_{n \in \mathbb{N}_{0}}$ ha sido producida por un proceso estocástico estacionario.

\footnotetext{
${ }^{3}$ En un proceso estacionario las propiedades estadísticas que lo caracterizan se mantienen constantes en el tiempo.
} 


\section{Referencias}

[Akin, 2010] Akin, E. (2010). The General Topology of Dynamical Systems (Graduate Studies in Mathematics). American Mathematical Society. (página 31).

[Almeida, 1990] Almeida, A. M. O. D. (1990). Hamiltonian Systems: Chaos and Quantization. Cambridge University Press. (página 35).

[Bai-lin, 1989] Bai-lin, H. (1989). Elementary Symbolic Dynamics and Chaos in Dissipative Systems. World Scientific. (página 36).

[Baker and Gollub, 1996] Baker, G. L. and Gollub, J. P. (1996). Chaotic Dynamics: An Introduction. Cambridge University Press. (página 33).

[Barnsley and Barnsley, 2006] Barnsley, M. and Barnsley, M. (2006). SuperFractals. Cambridge University Press. (página 33).

[Barreira, 2006] Barreira, L. (2006). Poincaré recurrence: old and new. In XIVth International Congress on Mathematical Physics. World Scientific. (página 37).

[Barreira, 2008] Barreira, L. (2008). Dimension and Recurrence in Hyperbolic Dynamics. Birkhäuser Verlag AG. (página 37).

[Barreira, 2012] Barreira, L. (2012). Ergodic Theory, Hyperbolic Dynamics and Dimension Theory. Springer Berlin Heidelberg. (páginas 31, 38).

[Barreira and Pesin, 2013] Barreira, L. and Pesin, Y. (2013). Introduction to Smooth Ergodic Theory (Graduate Studies in Mathematics). American Meteorological Society. (páginas 31,36 ).

[Borovkova, 1998] Borovkova, S. A. (1998). Estimation and prediction for nonlinear time series. PhD thesis, University of Groningen. (página 39).

[Bredon, 2013] Bredon, G. E. (2013). Topology and Geometry. Springer New York. (página 31).

[Brogliato et al., 2007] Brogliato, B., Maschke, B., Lozano, R., and Egeland, O. (2007). Dissipative systems. In Dissipative Systems Analysis and Control, pages 177-256. Springer London. (página 35).

[Casdagli, 1992] Casdagli, M. (1992). Chaos and deterministic versus stochastic nonlinear modelling. Journal of the Royal Statistical Society. Series B (Methodological), 54(2):303-328. (página 40). 
[Choe, 2006] Choe, G. H. (2006). Computational Ergodic Theory. Springer Berlin Heidelberg. (página 38).

[Devaney, 2018] Devaney, R. (2018). An Introduction To Chaotic Dynamical Systems. CRC Press. (página 32).

[Eckmann and Ruelle, 1985] Eckmann, J. P. and Ruelle, D. (1985). Ergodic theory of chaos and strange attractors. Reviews of Modern Physics, 57(3):617-656. (páginas $36,47,105,106,165,213)$.

[Falconer, 2013] Falconer, K. (2013). Fractal Geometry. John Wiley \& Sons. (página 33).

[Farmer et al., 1983] Farmer, J. D., Ott, E., and Yorke, J. A. (1983). The dimension of chaotic attractors. In The Theory of Chaotic Attractors, pages 142-169. Springer New York. (páginas 36, 114).

[Feigenbaum, 1983] Feigenbaum, M. J. (1983). Universal behavior in nonlinear systems. Physica D: Nonlinear Phenomena, 7(1-3):16-39. (página 33).

[Halmos, 2006] Halmos, P. R. (2006). Lectures on Ergodic Theory (AMS Chelsea Publishing). American Mathematical Society. (páginas 36, 38).

[Hilborn, 2001] Hilborn, R. C. (2001). Chaos and Nonlinear Dynamics: An Introduction for Scientists and Engineers. Oxford University Press. (página 36).

[Hirsch, 2012] Hirsch, M. W. (2012). Differential Topology. Springer New York. (página 31).

[Kantz and Schreiber, 2003] Kantz, H. and Schreiber, T. (2003). Nonlinear Time Series Analysis. Cambridge University Press. (páginas 36, 52, 55, 59, 65).

[Lee, 2010] Lee, J. M. (2010). Introduction to Topological Manifolds. Springer New York. (página 31).

[Lee, 2013] Lee, J. M. (2013). Introduction to Smooth Manifolds. Springer New York. (página 31).

[Lorenz, 1963] Lorenz, E. N. (1963). Deterministic nonperiodic flow. Journal of the Atmospheric Sciences, 20(2):130-141. (página 32).

[Mandelbrot, 1983] Mandelbrot, B. B. (1983). The Fractal Geometry of Nature. W. H. Freeman. (páginas 33, 71, 112).

[May, 1976] May, R. M. (1976). Simple mathematical models with very complicated dynamics. Nature, 261(5560):459-467. (página 32).

[Moon, 1992] Moon, F. C. (1992). Chaotic and Fractal Dynamics: Introduction for Applied Scientists and Engineers. Wiley. (página 86). 
[Pawelzik and Schuster, 1987] Pawelzik, K. and Schuster, H. G. (1987). Generalized dimensions and entropies from a measured time series. Physical Review A, 35(1):481-484. (página 36).

[Peitgen et al., 2013] Peitgen, H.-O., Jürgens, H., and Saupe, D. (2013). Chaos and Fractals. Springer New York. (página 33).

[Poincaré, 2013] Poincaré, H. (2013). Science and Method. Dover Publications. (página 29).

[Ruelle, 1976] Ruelle, D. (1976). A measure associated with axiom-a attractors. American Journal of Mathematics, 98(3):619-654. (página 40).

[Ruelle, 1989] Ruelle, D. (1989). Chaotic Evolution and Strange Attractors. Cambridge University Press. (páginas 31, 36, 40).

[Ruelle and Takens, 1971] Ruelle, D. and Takens, F. (1971). On the nature of turbulence. Comm. Math. Phys., 20(3):167-192. (páginas 32, 95).

[Sauer et al., 1991] Sauer, T., Yorke, J. A., and Casdagli, M. (1991). Embedology. Journal of Statistical Physics, 65(3-4):579-616. (páginas 39, 48, 51, 54, 75).

[Seely and Macklem, 2012] Seely, A. J. E. and Macklem, P. (2012). Fractal variability: An emergent property of complex dissipative systems. Chaos: An Interdisciplinary Journal of Nonlinear Science, 22(1):013108. (página 35).

[Seimenis, 2013] Seimenis, J. (2013). Hamiltonian Mechanics: Integrability and Chaotic Behavior. Volumen 331 de Nato Science Series B. Springer US. (página 31).

[Smale et al., 2003] Smale, S., Hirsch, M. W., and Devaney, R. L. (2003). Differential Equations, Dynamical Systems, and an Introduction to Chaos (Pure and Applied Mathematics). Academic Press. (página 31).

[Sprott, 2003] Sprott, J. C. (2003). Chaos and Time-Series Analysis. Oxford University Press. (páginas 34, 86, 96).

[Strogatz, 1994] Strogatz, S. H. (1994). Nonlinear Dynamics And Chaos. Studies in nonlinearity. Perseus Books Publishing, LLC. (páginas 32, 34, 91).

[Takens, 1981] Takens, F. (1981). Detecting strange attractors in turbulence. In Lecture Notes in Mathematics, pages 366-381. Springer Berlin Heidelberg. (páginas $39,39,47,47,48,51,54,54,75)$.

[Winfree, 1980] Winfree, A. T. (1980). The geometry of biological time (Biomathematics ; v. 8). Springer Verlag. (página 33). 



\section{Capítulo 3}

\section{Reconstrucción del espacio de estados. Método de los retardos}

La reconstrucción del espacio de estados consiste en reproducir una sucesión temporal $\{x(k \Delta t)\}_{k=1}^{N}$, compuesta por $N$ escalares, en un espacio euclidiano $\mathbb{R}^{m}$, donde $m$ identifica la dimensión de encaje o de reconstrucción y $\Delta t$ el tiempo de muestreo de los datos. ${ }^{1}$ A partir de la sucesión temporal escalar se construyen vectores $\mathbf{x}_{t} \in \mathbb{R}^{m}$ de la forma

$$
\mathbf{x}_{t}=[x(t), x(t+\tau), \ldots, x(t+(m-1) \tau)]^{T},
$$

donde los parámetros $\tau$ y $m$, el retardo (lag) y la dimensión de encaje (embedding dimension), respectivamente, han de fijarse en el proceso de reconstrucción.

Con esta práctica se espera que los puntos en el espacio $\mathbb{R}^{m}$ conformen un atractor que preserve las propiedades topológicas del atractor original, a priori desconocido. La principal técnica de reconstrucción se basa en el método de los retardos y suele calcular los parámetros $\tau$ y $m$ de forma empírica y, por tanto, no necesariamente se proporcionan estimaciones adecuadas, aun admitiéndose de por sí un alto grado de incertidumbre cuando se opera con datos reales. En este sentido se han planteado procedimientos que pretenden mejorar las estimaciones de $\tau$ y $m$, como es el caso de la longitud de la ventana temporal, $\tau_{w}=(m-1) \tau$, con $\tau=n \Delta t$, que abarca cada vector de estado y que, en cierta medida, está relacionada con la cantidad de información transferida desde la sucesión temporal al espacio de reconstrucción. Una vez evaluado el valor de $\tau_{w}$ más idóneo, mediante la dimensión de correlación puede verificarse la calidad de la reconstrucción, dado que es probable que la idoneidad de $\tau_{w}$ para una estimación acertada de la dimensión pueda extrapolarse a otras invariantes, como la entropía, los exponentes de Lyapunov, e incluso a análisis predictivos [Kugiumtzis, 1996].

Un sistema dinámico puede describirse como una variedad diferenciable $M d$ dimensional y un operador de evolución $f$, de forma que $f^{t}$ permite pasar del estado inicial $\mathbf{s}\left(t_{0}\right)$ al estado $\mathbf{s}\left(t_{0}+t\right)$, con $\mathbf{s}(t) \in M \equiv \mathbb{R}^{d}$. La variable tiempo $t$ puede ser continua o discreta [Casdagli et al., 1991]. Luego,

\footnotetext{
${ }^{1}$ Un encaje (embedding) comprende una transformación de coordenadas biyectiva continuamente diferenciable, con una inversa también continuamente diferenciable (difeomorfismo).
} 


$$
\mathbf{s}\left(t+t_{0}\right)=f^{t}\left(\mathbf{s}\left(t_{0}\right)\right)
$$

donde $\mathbf{s}\left(t_{0}\right)$ representa el estado del sistema en el instante de tiempo $t_{0}$. En ausencia de ruido puede establecerse una relación directa entre una sucesión temporal $D$ dimensional y el sistema dinámico,

$$
x(t)=h(\mathbf{s}(t)),
$$

donde $h: M \rightarrow \mathbb{R}^{D}$ identifica la función de medición. Normalmente, $D<d$, y, a menudo, $D=1$. En otras palabras, a nivel experimental no se suele disponer de las $d$ variables que realmente determinan la evolución temporal del sistema físico bajo estudio. Únicamente se miden algunas variables (observables), habitualmente una sola variable $D=1$, a intervalos regulares de tiempo $\Delta t$, registrándose la sucesión de valores adquiridos como $x\left(t_{0}\right), x\left(t_{1}\right), \ldots$, con $x\left(t_{i}\right) \in \mathbb{R}$ y $t_{i}=t_{0}+i \Delta t$. Esta sucesión de valores adquiridos, en el caso de una sola variable, es lo que ya se denominó anteriormente sucesión temporal escalar. La función de medición $h$ comporta una proyección del espacio de estados $\mathbb{R}^{d}$ en el eje real $\mathbb{R}$, esto es,

$$
h \equiv \pi: \mathbb{R}^{d} \rightarrow \mathbb{R} .
$$

En resumidas cuentas una sucesión temporal escalar es unidimensional $(D=1)$, y, por consiguiente, proporciona una descripción incompleta o parcial del sistema físico, a saber, del sistema dinámico y de su evolución temporal. En este sentido se requiere algún mecanismo que posibilite inferir muchas propiedades del sistema dinámico a partir de la única información disponible, la información contenida en la sucesión temporal adquirida. En cierta medida lo que se pretende es reconstruir el espacio de estados del sistema dinámico a partir de la sucesión temporal de que se dispone. La figura 3.1 ilustra el planteamiento del problema.

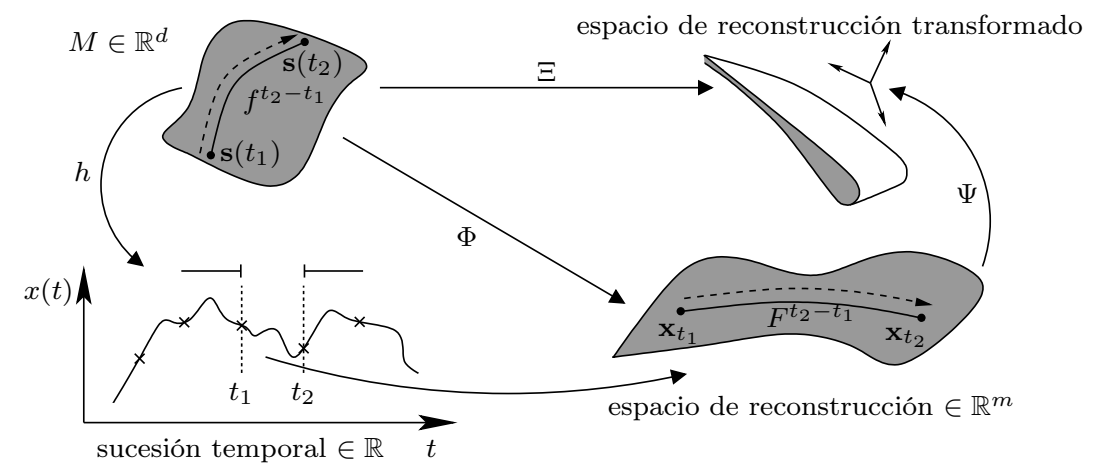

Figura 3.1: Planteamiento del problema de reconstrucción del espacio de estados.

El análisis no lineal de sucesiones temporales, desde la perspectiva de la teoría de los sistemas dinámicos, reclama, como un paso previo e ineludible, la reconstrucción del espacio de estados. Típicamente, $f$ y $h$ se desconocen, por lo que será virtualmente imposible recrear los estados originales. Por tanto, debería construirse un espacio de estados en cierta manera equivalente al espacio de estados originario. Este espacio 
de estados reconstruidos recibe el nombre de espacio de fases [Tsonis and Elsner, 1988]. El espacio de fases admite un análisis cualitativo (diagrama de fases) y cuantitativo, una caracterización estadística del sistema dinámico.

\subsection{El porqué de la reconstrucción de estados}

La reconstrucción del espacio de estados no es una idea reciente; ya en 1927 el estadístico escocés George Udny Yule había planteado este procedimiento para analizar la periodicidad de las manchas solares [Yule, 1927]. En la teoría de los sistemas dinámicos la reconstrucción de estados fue introducida independientemente por Norman Packard et al. [Packard et al., 1980] y Floris Takens [Takens, 1981], con las contribuciones del matemático francés David Ruelle. En [Packard et al., 1980] se concibe un esquema vectorial que posibilitaba encajar la sucesión temporal en un espacio $m$-dimensional, con $m>d$. La representación vectorial $\mathbf{x}_{t}$ de la sucesión temporal escalar, atendiendo a la ecuación (3.1), conforma una aproximación más integral del estado del sistema, en el instante $t$, que $x(t)$. El mapeo $\Phi$ entre el espacio de estados $\mathbb{R}^{d}$ y el espacio de fases $\mathbb{R}^{m}$ obedece a

$$
\Phi \equiv \pi^{(m)}: \mathbb{R}^{d} \rightarrow \mathbb{R}^{m} .
$$

Floris Takens [Takens, 1981] y Ricardo Mañé [Mañé, 1981] han demostrado que este procedimiento reconstruye en casi todos los casos el espacio de estados original siempre que $m>2 d_{F}+1$, siendo $d_{F}$ la dimensión fractal del atractor, es decir, el mapeo $\Phi$, restringido a un posible atractor $A$ del sistema dinámico, comprende una biyección continuamente diferenciable, o sea, una correspondencia uno a uno entre el atractor real y el atractor reconstruido. Si $m>d_{F}$, la dimensión del atractor reconstruido coincide con la dimensión del atractor original [Eckmann and Ruelle, 1985].

En principio se puede optar por cualquier valor de $m$ y de $\tau$, siempre que se disponga de un número infinito de datos de precisión infinita. En realidad este escenario es una quimera y la selección óptima de los parámetros $m$ y $\tau$ es crucial para una reconstrucción fidedigna del espacio de estados. Si el producto $(m-1) \tau$ es demasiado grande, las componentes $x(t)$ y $x(t+(m-1) \tau)$ del vector reconstruido $\mathbf{x}_{t}$ posiblemente no estén correlacionadas, lo que se traduce en una sobreestimación de la dimensión. De igual forma, si el producto $(m-1) \tau$ es demasiado pequeño, las componentes $x(t)$ y $x(t+(m-1) \tau)$ del vector reconstruido $\mathbf{x}_{t}$ probablemente sean casi idénticas, máxime si el intervalo entre muestras es muy reducido, lo que conduce a que el atractor se disponga geométricamente sobre una línea diagonal o bisectriz. Además, si el valor de $\tau$ es muy pequeño, aun con un valor de $m$ grande, significa que $x(t) \approx x(t+\tau)$, esto es, componentes consecutivas del vector de reconstrucción son redundantes.

Con estos antecedentes, ni el valor de $\tau$ debiera ser menor que un cierto tiempo de decorrelación característico, ni el producto $(m-1) \tau$ debiera ser mucho mayor que este tiempo de decorrelación. Una estrategia para resolver el tiempo de decorrelación deriva del primer paso por cero de la función de autocorrelación de los datos, si bien esta táctica únicamente contempla las correlaciones lineales entre los datos, 
de ahí que suela recurrirse al primer mínimo de la información mutua, ya que esta última atiende las correlaciones de un modo más genérico, tanto lineales como no lineales [Fraser and Swinney, 1986]. En el caso de la dimensión de encaje $m$, su valor puede obtenerse mediante una regla topológica, por la que, una vez alcanzada la dimensión óptima, las relaciones de vecindad son preservadas con independencia de que se incremente su valor [Liebert et al., 1991].

El mecanismo de reconstrucción se ha convertido en una práctica muy extendida, puesto que permite analizar el comportamiento autoorganizado de sistemas muy complejos sin necesidad de conocer el verdadero estado del sistema en un instante de tiempo determinado, esto es, con una sola variable es factible recuperar la dinámica presente en el sistema físico; dado que la evolución de una variable está influenciada por cualquier otra variable con la que esté interactuando, sus valores deben estar contenidos hasta cierto punto en la historia de la otra variable. De algún modo su marca debe de estar ahí, según ciertas reflexiones de J. Doyne Farmer en [Gleick, 2012].

\subsubsection{Inconvenientes en un escenario real: proceso de reconstruc- ción}

En un escenario real la aportación más importante de la reconstrucción del espacio de estados a la teoría de los sistemas dinámicos es su capacidad para preservar invariantes geométricas, como la dimensión de un atractor o los exponentes de Lyapunov de una trayectoria. El pasado y el futuro de cualquier sucesión temporal contienen información de aquellas variables de estado no observadas, pero latentes, que definen el estado presente del sistema dinámico. Esta información pasada y futura puede registrarse en un vector cuyas componentes son muestras desplazadas en el tiempo, a intervalos regulares, del mismo observable, esto es,

$$
\mathbf{x}_{t}=\left[x\left(t-\tau m_{\mathrm{p}}\right), \ldots, x(t), \ldots, x\left(t+\tau m_{\mathrm{f}}\right)\right]^{T},
$$

donde $m_{\mathrm{p}}$ identifica el número de muestras tomadas del pasado, y $m_{\mathrm{f}}$ el número de muestras tomadas del futuro. La dimensión del vector $\mathbf{x}_{t}$ asciende a $1+m_{\mathrm{p}}+m_{\mathrm{f}}$. Si $m_{\mathrm{f}}=0$, se dice que la reconstrucción es predictiva. La separación $\tau$ entre coordenadas consecutivas se denomina tiempo de retardo (lag time).

A diferencia de Norman Packard et al. [Packard et al., 1980], quienes evaluaron numéricamente las invariantes del proceso de reconstrucción de estados, Floris Takens [Takens, 1981], y, posteriormente, Tim Sauer et al. [Sauer et al., 1991] estudiaron matemáticamente las características del mapa de reconstrucción $\Phi$, que mapea estados del sistema dinámico $d$-dimensional a vectores reconstruidos $m$-dimensionales, conforme a

$$
\Phi(\mathbf{s}(t))=\left[h\left(f^{-\tau m_{\mathrm{p}}}(\mathbf{s}(t))\right), \ldots, h(\mathbf{s}(t)), \ldots, h\left(f^{\tau m_{\mathrm{f}}}(\mathbf{s}(t))\right)\right]^{T} .
$$

Floris Takens demostró que, en general, el mapa $\Phi$ ejerce como un encaje cuando $m \geqslant 2 d+1$. Si $\Phi$ es un encaje, resulta factible inducir un operador de evolución continuamente diferenciable $F$ en el espacio de vectores reconstruidos, esto es, 


$$
F^{t}\left(\mathbf{x}_{t}\right)=\Phi \circ f^{t} \circ \Phi^{-1}\left(\mathbf{x}_{t}\right) .
$$

Dado que $F$ es equivalente al operador dinámico $f$, pueden emplearse los estados reconstruidos para inferir $F$, y, a partir de su derivación, es posible extraer propiedades de la dinámica original, como su dimensión, su entropía, su potencial predictivo, etc. La idoneidad del proceso de reconstrucción actúa oportunamente en un contexto abstracto, con unas condiciones de entorno muy controladas. En un escenario real, en el que los datos son escasos y contienen ruido, el valor de $\tau$ se convierte en un factor crítico, pues la presencia de ruido perturba la localización exacta de los estados del sistema dinámico y la precisión con la que se especifica un estado reconstruido contribuye aún más a la desubicación de los estados primitivos. Por tanto, la correcta reconstrucción de los estados se ve ensombrecida por los siguientes factores:

1. Ruido observacional. También denominado ruido de medición. Los instrumentos de medida introducen ruido en las mediciones adquiridas. Típicamente este ruido es independiente e idénticamente distribuido. Si el tiempo entre muestras es muy pequeño, el ruido puede estar correlacionado, de ahí que haya que garantizar que $\tau$ sea mayor que un cierto tiempo de correlación. Con cada medición de la sucesión temporal, lo que realmente se registra es

$$
x(t)=\tilde{x}(t)+\xi(t),
$$

donde $\tilde{x}(t)$ representa la verdadera medida, a saber, $x(t)=h(\mathbf{s}(t))$, y $\xi(t)$ denota la contribución del ruido en el instante de tiempo $t$ en el que se efectúa la medición.

2. Ruido dinámico. El entorno en que se desenvuelve el sistema dinámico perturba de alguna manera todo estado $\mathbf{s}(t)$. Los sistemas físicos no suelen operar de forma aislada, de ahí que la influencia externa provoque que la evolución del estado $\mathbf{s}(t)$ no sea determinista. En este sentido el operador de evolución $f$ es considerado estocástico.

3. Error de estimación. Normalmente se desconocen $f$ y $h$, por lo que ha de estimarse la dinámica del sistema a partir del espacio de vectores reconstruidos; en este sentido la aproximación es imperfecta si se dispone de un número limitado de datos, como es el caso en la mayoría de las situaciones experimentales.

En definitiva el ruido siempre está presente. Si se proyecta un estado de un espacio $d$-dimensional en un espacio $D$-dimensional, con $D<d$, se pierde información. Es posible recuperar parte de esa información perdida de mediciones pasadas y futuras. No obstante, si la incertidumbre del vector reconstruido es mayor que la de las mediciones individuales, se ha amplificado el ruido, lo que convierte al sistema reconstruido en menos determinista aún. Por tanto, la reconstrucción del espacio de estados se basa en la transferencia de información que se cursa desde variables no observadas a aquellos observables medidos, esto es, el hecho de no capturar más información del acoplamiento entre variables del sistema dinámico hace que el sistema reconstruido parezca menos determinista de lo que en realidad es. Dicho de otra 
manera, en el sistema reconstruido se está amplificando el ruido. La amplificación del ruido se supedita a varias causas:

1. La función de medición. La observación de una variable puede suministrar más información que la medida de otra variable del sistema dinámico; todo depende del grado de acoplamiento entre las variables que conforman el sistema dinámico bajo estudio.

2. El método de reconstrucción. Una óptima reconstrucción del espacio de estados amplifica menos el ruido que cualquier otra reconstrucción del espacio de estados más defectuosa. En este sentido una elección acertada de los parámetros $\tau$ y $m$ decanta la balanza hacia una buena reconstrucción o, por el contrario, hacia una reconstrucción incompleta.

3. El propio sistema dinámico. La amplificación del ruido se subordina al flujo de información entre los diferentes grados de libertad que rigen la dinámica del sistema físico; este flujo de información puede determinarse por medio de la dimensión y de los exponentes de Lyapunov.

La amplificación del ruido y la estimación del error inciden negativamente en la predicción de futuros estados del sistema dinámico, como se aprecia en la figura 3.2. Ambos fenómenos derivan del proceso de reconstrucción y causan errores de predicción, si bien la estimación del error tiende a cero cuando se dispone de un número ilimitado de datos. Por consiguiente, en presencia de muchos datos el error de predicción se debe únicamente al ruido. Aun con un modelo perfecto, es decir, con una aproximación muy precisa, todavía persiste un error de predicción debido al ruido, estableciéndose un límite a la capacidad predictiva del sistema reconstruido. Este límite constituye una frontera entre el determinismo subyacente al sistema físico y el comportamiento característico de un proceso aleatorio.
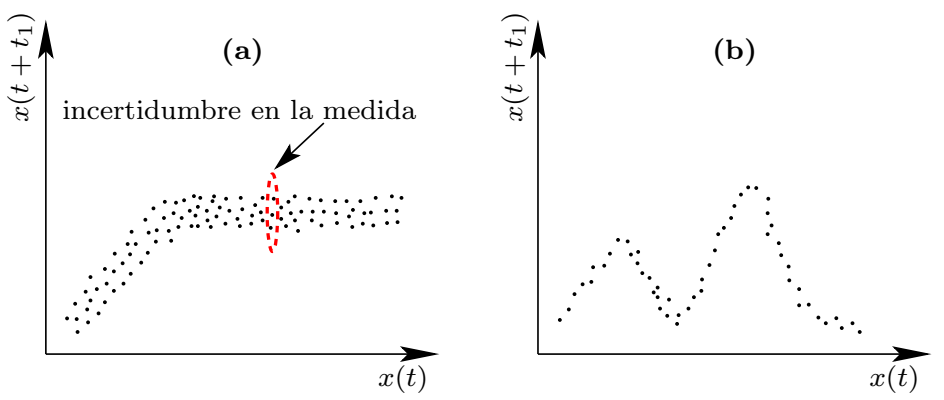

Figura 3.2: La amplificación del ruido y la estimación del error en dos escenarios ilustrativos [eje de abscisas, estado del sistema en el instante de tiempo $t$; eje de ordenadas, estado en el instante $t+t_{1}$ ]. En (a) el grosor de la distribución de puntos es sintomático de una amplificación de ruido significativa; en (b) la dinámica funcional sinuosa de los puntos es más compleja, lo que, en presencia de pocos puntos, podría llevar a errores de predicción notorios.

En algunos casos el proceso de reconstrucción no solo contempla la posibilidad de sacar a la luz la información contenida en el acoplamiento dinámico entre las 
variables de estado del sistema físico, a partir de muestras desplazadas en el tiempo de un único observable, sino también mecanismos que posibilitan la transformación de coordenadas, como, por ejemplo, la diferenciación o la descomposición en valores singulares, para reducir la dimensión del espacio de reconstrucción. Así, los nuevos estados reconstruidos, tras la transformación $\Psi$, quedan como

$$
\mathbf{y}_{t}=\Psi\left(\mathbf{x}_{t}\right) .
$$

Recapitulando, el proceso de reconstrucción puede descomponerse en dos partes diferenciadas, una primera parte determina el sistema de coordenadas de reconstrucción, a partir de los datos en bruto de la sucesión temporal, y, por ende, la información rescatada de los datos; la segunda parte determina cómo operar con la información recuperada de los datos, resaltando que la transformación del sistema de coordenadas no reduce la amplificación del ruido, a lo sumo permanece igual. De esta forma, el mapa

$$
\Xi:=\Psi \circ \Phi
$$

mapea el estado original $\mathbf{s}$ a un vector reconstruido, y transformado, $\mathbf{y}$. No en pocas ocasiones, la transformación $\Psi$ se simplifica al máximo, esto es, la transformación se resume en una simple función identidad id, de forma que

$$
\Psi\left(\mathbf{x}_{t}\right)=\operatorname{id}\left(\mathbf{x}_{t}\right)=\mathbf{x}_{t}=\mathbf{y}_{t} .
$$

Con la función identidad se asegura que el ruido es idéntico en ambos espacios, el espacio de reconstrucción y el espacio transformado.

En principio todas las reconstrucciones del espacio de estados que son un encaje serían equivalentes entre sí. Sin embargo, cómo se escogen las coordenadas de los vectores de reconstrucción influye en la habilidad del sistema reconstruido para efectuar predicciones. Una reconstrucción imperfecta amplifica el ruido e incrementa el error de estimación. Normalmente la descripción óptima de un espacio de reconstrucción obliga a buscar una solución intermedia entre ventanas temporales grandes y pequeñas. Una ventana temporal grande reduce el ruido observacional, en detrimento del error de estimación; en cambio, con una ventana temporal pequeña el efecto dominante es el ruido observacional. En sistemas caóticos el ruido se amplifica conforme se hace más grande el mayor exponente de Lyapunov o la dimensión del atractor. Además, cualquier proyección del sistema en espacios de dimensión más baja dispara el efecto del ruido observacional, lo que impide reconstruir los estados fielmente y, por tanto, el horizonte de predicción se reduce al mínimo, incluso por debajo del tiempo de Lyapunov. En estas condiciones un sistema caótico y un sistema aleatorio son indistinguibles.

Los teoremas matemáticos que examinan analíticamente los mapas de reconstrucción, y caracterizan su comportamiento, responden a pruebas de existencia [Pecora et al., 2009], es decir, corroboran la existencia de encajes que posibilitan una correspondencia unívoca entre los estados originarios y los estados reconstruidos [Takens, 1981, Sauer et al., 1991], pero no plantean métodos funcionales que permitan obtener los valores óptimos de $\tau$ y $m$ a partir de una sucesión temporal escalar. Más bien son criterios heurísticos, sobre la base de ciertos estadísticos, los que prevalecen en 
el proceso de selección de los parámetros $\tau$ y $m$. Por lo general, la determinación de los parámetros se lleva a cabo de forma independiente, es decir, primero se evalúa la mejor opción para el parámetro $\tau$ y después, separadamente, se estima el valor adecuado de $m$. En cualquier caso se han ideado múltiples procedimientos para intentar inferir los párametros de reconstrucción, si bien en todos ellos los criterios que establecen qué valores de $\tau$ y $m$ son los más apropiados no son muy rigurosos, salvo en el contexto de soluciones específicas $a d h o c$, y se someten al juicio y a la valoración del experimentador, en virtud del conocimiento, en calidad de especialista en la materia, de que dispone sobre el sistema físico que subyace a la sucesión temporal de interés. Obviamente, los valores de $\tau$ y $m$ obtenidos están sujetos a muchos condicionantes, que, en muchos casos, ignoran las múltiples escalas en que se desarrolla la dinámica de cualquier sistema complejo, aparte de otras deficiencias computacionales debidas a ciertos inconvenientes inherentes a las habituales sucesiones temporales disponibles, su longitud limitada y el ruido sempiterno [Pecora et al., 2009, Abarbanel et al., 1993, Kantz and Schreiber, 2003].

\subsubsection{Métodos de reconstrucción}

Las diferentes metodologías se basan en nociones extraídas de diversas disciplinas matemáticas, como la topología, la teoría de los sistemas dinámicos, la teoría de la información y la teoría de la aproximación en métodos numéricos. Con estas herramientas, aparte de la interpretación de los resultados, sujetos en muchos casos a la experiencia del estudioso en la materia de la que se extraen los datos, ha de ser factible identificar la naturaleza del comportamiento que exhiben los datos, a fin de plantear diversos modelos con los que predecir conductas futuras, e, incluso, yendo un paso más allá, controlar las pautas dinámicas del sistema, dirigiéndolo hacia configuraciones «seguras».

En un entorno experimental, como se introdujo en líneas precedentes, se suele disponer de una sucesión temporal escalar $\left\{x\left(t_{i}\right)\right\}_{i=0}^{N-1}$, con $t_{i}=t_{0}+i \Delta t, N$ observaciones (números reales) de un experimento, que inicia en un instante de tiempo $t_{0}$, adquiridas a intervalos regulares de tiempo $\Delta t$, siendo $\Delta t$ el tiempo de muestreo en sistemas continuos, o el intervalo de tiempo discreto, en el caso de sistemas de tiempo discreto. Del sistema dinámico se desconoce la dimensión del espacio de estados, el operador de evolución $f$ entre estados, así como la función de medición $h$; únicamente se cuenta con las mediciones u observaciones y el tiempo $\Delta t$ entre ellas. Dado que se ignora la dinámica del sistema físico, no es posible a priori reconstruir fielmente el atractor original del que se desprenden las observaciones adquiridas. Por tanto, ha de definirse un espacio de reconstrucción que permita, a partir de los datos disponibles, recrear un atractor que preserve las características invariantes del desconocido atractor original. La dimensión del espacio reconstruido $m$ difiere normalmente de la dimensión del espacio en que se desenvuelve el atractor original, $\left[d_{F}\right]+1$, donde $[\cdot]$ identifica la parte entera de la dimensión fractal del atractor. El método más sencillo del que pueden derivarse vectores de estado responde al método de los retardos (delay method). Un vector de estado en un espacio de reconstrucción de dimensión $m$ consta de $m$ observaciones separadas $\tau$ unidades de tiempo entre sí, esto es, 


$$
\mathbf{x}_{t}=[x(t), x(t+\tau), \ldots, x(t+(m-1) \tau)]^{T},
$$

donde $\tau$ es un múltiplo entero de $\Delta t$ e identifica el retardo temporal entre coordenadas consecutivas. El nuevo espacio de reconstrucción, llamado espacio de fases [Tsonis and Elsner, 1988], estará compuesto por una colección

$$
\left\{\mathbf{x}_{k}\right\}_{k=1}^{N-(m-1) \tau}
$$

de vectores de estado reconstruidos, concretamente por $N-(m-1) \tau$ vectores. En suma el proceso de reconstrucción de vectores de estado consiste en determinar la dimensión de reconstrucción $m$ y el retardo $\tau$ entre las coordenadas consecutivas de cada vector.

Las diferentes propuestas de reconstrucción del espacio de estados, que describen cómo obtener las componentes de los vectores de estado, no proporcionan resultados similares. De entre los planteamientos más extendidos, en el ámbito de los sistemas dinámicos caóticos, aparte del método de los retardos, sobresalen el análisis de componentes principales [Broomhead and King, 1986, Fraser, 1989b], y el método de las derivadas, investigado numéricamente por Norman Packard et al. [Packard et al., 1980]. En este último escenario las sucesivas coordenadas de los vectores de estado se corresponden con derivadas de orden superior, esto es,

$$
\mathbf{x}_{t}=\left[x(t), \hat{x}^{\prime}(t), \ldots, \hat{x}^{(m-1)}(t)\right]^{T},
$$

donde $\hat{x}^{(i)}(t)$ denota la aproximación numérica a la $i$-ésima derivada de $x(t)$. Siempre que $m$ es suficientemente grande, el método de las derivadas posibilita la concreción de un encaje válido. Además, el hecho de que se hayan desarrollado múltiples algoritmos para computar numéricamente las correspondientes derivadas, suministra toda una familia de métodos en función del algoritmo utilizado. Las coordenadas de los vectores de estado de los tres métodos comentados, el anális de componentes principales, el método de las derivadas y el método de los retardos, se relacionan entre sí mediante transformaciones lineales. Sin embargo, las coordenadas de los vectores de estado, según el método de los retardos, se relacionan con las variables de estado del espacio de estados original por medio de una transformación no lineal, lo que acrecienta su grado de verosimilitud [Fraser, 1989a], respaldando su amplio uso en el análisis no lineal de sucesiones temporales. De hecho, el método de los retardos asegura que la relación señal a ruido de cada componente del vector de estado sea idéntica. Por el contrario, obliga a seleccionar un valor de retardo $\tau$ entre las componentes del vector. Si $\tau$ adopta un valor muy pequeño, las trayectorias del espacio de reconstrucción se disponen a lo largo de la línea identidad. Este fenómeno recibe el nombre de redundancia. Si $\tau$ adopta un valor muy grande, las coordenadas de los vectores de estado se desconectan dinámicamente entre sí, se pierde la relación de causalidad, provocando que un objeto geométrico aparentemente simple se distorsione en extremo. Este fenómeno se denomina irrelevancia [Casdagli et al., 1991]. 


\subsection{Método de los retardos}

El método de los retardos se basa en el teorema de Takens [Takens, 1981]. ${ }^{2} \mathrm{El}$ teorema de Takens garantiza la existencia de una dimensión de encaje $m$ que preserva las características invariantes del sistema físico subyacente, siempre que se disponga de un número ilimitado de datos libres de ruido. Floris Takens demostró que, bajo estas condiciones ideales, es suficiente con que $m \geqslant 2\left[d_{F}\right]+1$. En el teorema no se menciona el efecto que $\tau$ y $\Delta t$ pudieran tener en la calidad de la reconstrucción, dado que en un entorno ideal $\tau$ y $\Delta t$ pueden adoptar cualquier valor arbitrario. El teorema en cierta medida garantiza la ausencia de autointersecciones en un atractor embebido en un espacio $m$-dimensional. En presencia de datos reales $\tau$ y $\Delta t$ influyen decisivamente en la calidad de la reconstrucción. La condición $m \geqslant 2\left[d_{F}\right]+1$ puede ser suficiente en configuraciones muy dispares, pero en ocasiones es posible conseguir una reconstrucción efectiva con $m \geqslant\left[d_{F}\right]+1$. A modo de guía [Sauer and Yorke, 1993, Ding et al., 1993], conforme a un sólido formalismo matemático, Floris Takens [Takens, 1981] demostró que el encaje es válido siempre que $m=2 d_{\text {top }}+1$, donde $d_{\text {top }}$ representa la dimensión topológica (entera), si el atractor es una variedad. Ricardo Mañé [Mañé, 1981] demostró que el encaje es válido siempre que $m>2 d_{H}+1$, donde $d_{H}$ representa la dimensión de Hausdorff (no entera), si el atractor no es una variedad. Finalmente, Tim Sauer et al. [Sauer et al., 1991] demostraron que el encaje es válido siempre que $m>2 d_{C}$, donde $d_{C}$ representa la dimensión de capacidad (no entera).

En realidad, con un número finito de datos con ruido, las estimaciones de las diferentes invariantes del sistema dinámico, como la dimensión del atractor, los exponentes de Lyapunov, o su entropía, se supeditan fuertemente a los valores que adoptan $\tau$ y $m$, de ahí que todo análisis no lineal de una sucesión temporal que se precie ha de descansar en una selección apropiada de los parámetros $\tau$ y $m$, para asegurar así una reconstrucción óptima del espacio de estados.

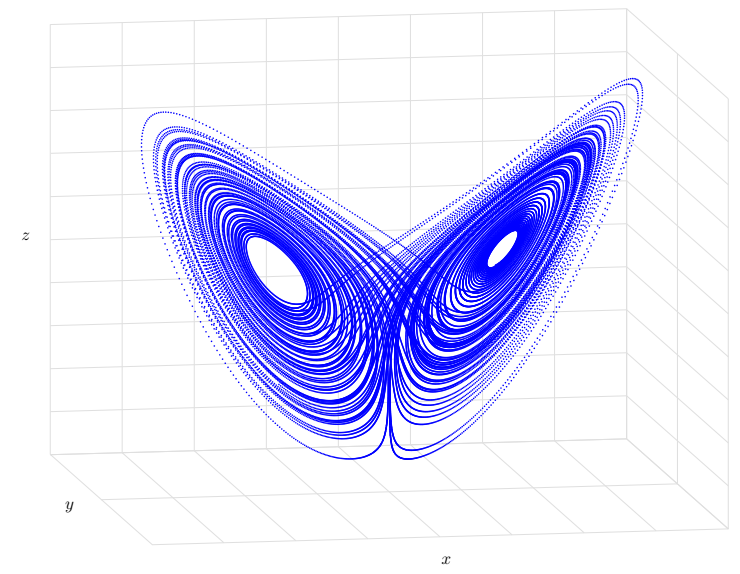

Figura 3.3: Espacio de estados del atractor de Lorenz.

\footnotetext{
${ }^{2} \mathrm{~A}$ veces se hace referencia indistintamente al teorema de Whitney, dado que el matemático norteamericano Hassler Whitney fue el primero en demostrar que una variedad $n$-dimensional continuamente diferenciable puede ser embebida en $\mathbb{R}^{2 n+1}$ [Whitney, 1936].
} 
Para facilitar la comprensión y el alcance de los conceptos de este capítulo se incorporan algunas ilustraciones explicativas de los resultados que se obtienen cuando se aplican los métodos de selección de los parámetros $\tau$ y $m$ a la variable dinámica $x$ de un sistema físico muy habitual en el análisis no lineal, el sistema de Lorenz, cuyo espacio de estados, la afamada mariposa del atractor de Lorenz, aparece reflejado en la figura 3.3.

\subsubsection{Selección del parámetro $\tau$}

La selección del parámetro $\tau$ no es trivial, pues ni siquiera se contempla su definición en los teoremas del método de los retardos y su efecto es crítico en el análisis posterior de cualquier sistema dinámico reconstruido. Valores pequeños de $\tau$ causan que las componentes de los vectores de estado $\mathbf{x}_{t}$ sean similares (redundantes), lo que explica por qué el atractor reconstruido se extienda prácticamente a lo largo de una diagonal en cualquier diagrama de fases, aun en presencia de mucho ruido. En este sentido, si los datos disponibles contienen ruido, ha de comprobarse que en la ventana temporal que acapara cada vector de estado, $(m-1) \tau$ observaciones, o $(m-1) \tau \Delta t$, en unidades de tiempo, la variación de la señal sea superior a la amplitud del ruido; en caso contrario, la información del vector de estado carece de significación dinámica. Por tanto, el valor mínimo de $\tau$ está condicionado por el ruido, esto es, el valor mínimo de $\tau$ coincide con el tiempo que invierte la función de autocorrelación normalizada en descender hasta

$$
1-\frac{\sigma_{\text {ruido }}^{2}}{\sigma_{\text {señal }}^{2}}
$$

siendo $\sigma_{\text {ruido }}^{2}$ la amplitud del ruido, y $\sigma_{\text {señal }}^{2}$ la varianza de los datos presentes en la sucesión temporal [Kantz and Schreiber, 2003]. De alguna manera, el propósito de $\tau$ es afianzar la mayor independencia temporal posible entre las componentes de cada vector de estado $\mathbf{x}_{t}$, sin destruir el determinismo subyacente en la evolución temporal de los estados del sistema físico, es decir, sin perder la información, contenida en los datos, que rige la conexión que se establece en la progresión temporal de los estados dinámicos. Si esta conexión se rompiera, las relaciones de causalidad entre los estados desaparecerían y los diferentes estados no estarían gobernados por una ley de evolución temporal determinista, de ahí que tampoco sea juicioso optar por valores grandes de $\tau$, con objeto de no deshacer el determinismo implícito en el espacio de estados.

\subsubsection{Método de llenado del espacio}

Un primer procedimiento, de carácter empírico (método científico de ensayo y error) y geométrico, estriba en representar visualmente, en un diagrama de fases, los vectores de estado con diferentes valores de $\tau$ para una dimensión de encaje $m$ suficientemente grande, asegurándose que el atractor pueda desplegarse en toda su extensión, como se aprecia en la figura 3.4. Es el denominado método de llenado del espacio (space-filling method) [Fraedrich, 1986]. Carece de toda fundamentación rigurosa y la interpretación de los resultados está sujeta a criterios muy subjetivos. 
Para valores pequeños de $\tau$ los vectores de estado se estructuran a lo largo de una línea identidad algo distorsionada o ruidosa, pues las componentes de los vectores de estado son aproximadamente iguales (ver figura 3.4a). A medida que aumenta el valor de $\tau$ los vectores de estado van organizándose en torno a una nube de puntos en expansión, en la que el determinismo solo resulta visible a pequeñas escalas (ver figuras 3.4b y 3.4c). La expansión de esta nube de puntos, el llenado del espacio, alcanzará un límite máximo, a partir del cual un incremento del valor de $\tau$ no aportaría mayor capacidad expansiva, más bien destruiría la conectividad inherente a todo proceso determinista (ver figura 3.4d), lo que se traduce en una mayor complejidad aparente del objeto geométrico representado en el diagrama de fases. Se han definido dos estadísticos que determinan el valor de $\tau$ a partir de consideraciones geométricas, el factor de llenado del espacio de reconstrucción [Buzug et al., 1990] y la desviación de la diagonal [Rosenstein et al., 1994], aparte de otros métodos geométricos que persiguen maximizar la expansión del atractor respecto a la diagonal [Buzug and Pfister, 1992, Kember and Fowler, 1993].

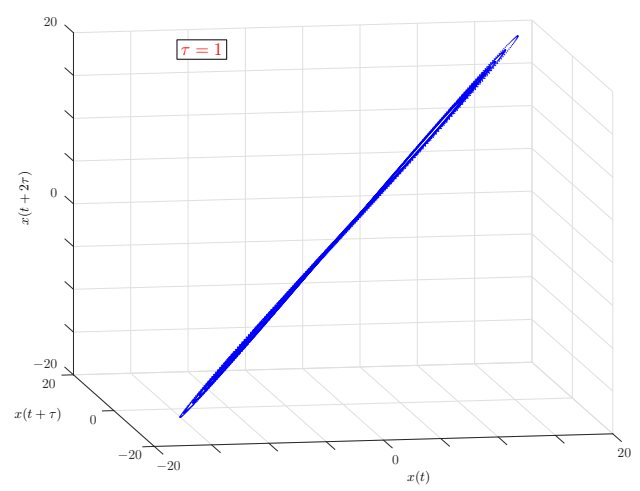

(a) $\operatorname{Con} \tau=1$.

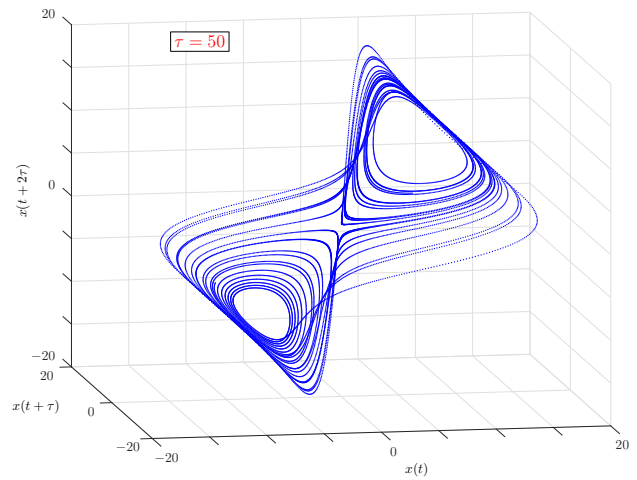

(c) Con $\tau=50$.

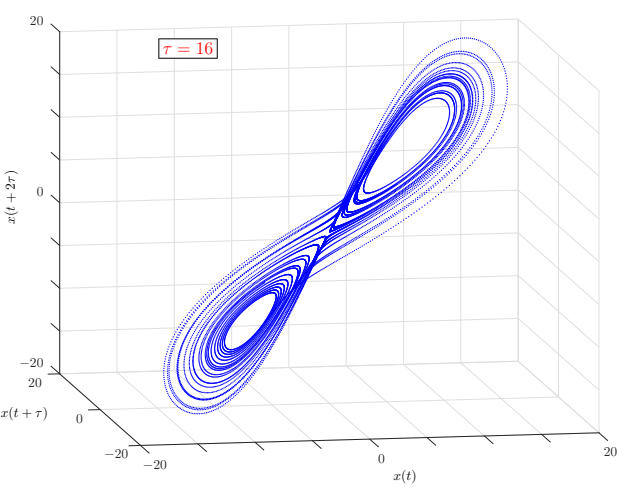

(b) Con $\tau=16$.

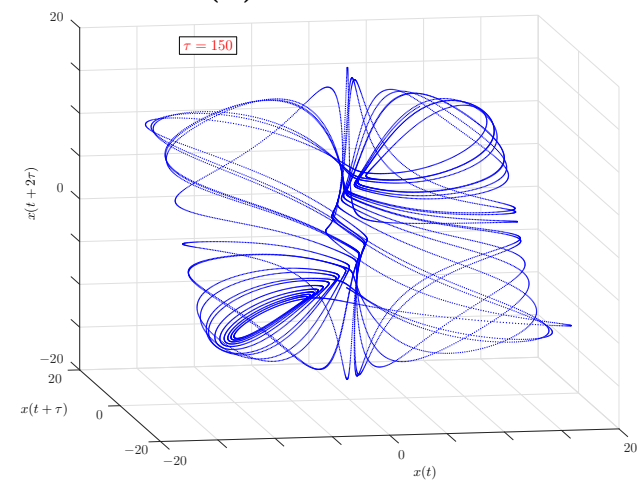

(d) $\operatorname{Con} \tau=150$.

Figura 3.4: Reconstrucción del espacio de estados a partir de la variable dinámica $x$ del atractor de Lorenz para diferentes valores de $\tau$. Puede observarse cómo a medida que aumenta $\tau$ el atractor va desplegándose en toda su magnitud hasta un punto en que se desvanece toda causalidad dinámica. 


\subsubsection{Función de autocorrelación}

Una alternativa muy extendida en la selección del parámetro $\tau$ se inclina por la función de autocorrelación; el primer paso por cero de la función de autocorrelación se convierte en el valor de $\tau$ que determina la mínima correlación lineal entre las componentes de cada vector de estado $\mathbf{x}_{t}$. Sea $\{x(t)\}_{t=0}^{N-1}$ una sucesión temporal compuesta por $N$ observaciones. La función de autocorrelación $R(\tau)$ para un determinado $\tau$ obedece a

$$
R(\tau)=\frac{\sum_{t=0}^{N-1-\tau}[x(t)-\langle x(t)\rangle][x(t+\tau)-\langle x(t)\rangle]}{\sum_{t=0}^{N-1}[x(t)-\langle x(t)\rangle]^{2}},
$$

donde $\langle x(t)\rangle$ identifica la media de los datos presentes en la sucesión temporal. Además del primer paso por cero de la función de autocorrelación (ver figura 3.5), no es infrecuente encontrarse, en función de la naturaleza de los datos y de criterios más bien subjetivos, otras preferencias para el valor de $\tau$, como el tiempo de autocorrelación en el caso de atractores climáticos [Tsonis and Elsner, 1988], a saber, el tiempo que transcurre hasta que la función de autocorrelación desciende a un valor de $1 / e \approx 0,37$, o el tiempo para el que la función de autocorrelación alcanza su primer mínimo.

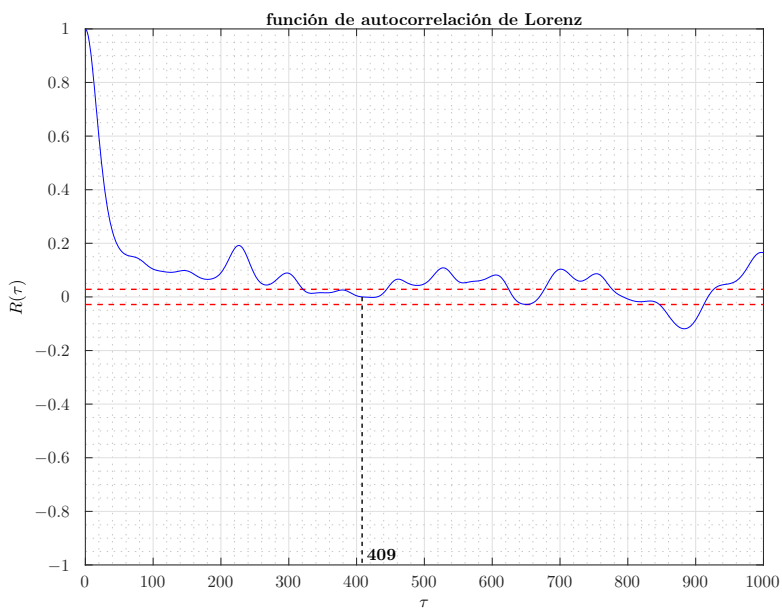

Figura 3.5: Función de autocorrelación del atractor de Lorenz para la variable dinámica $x$, en la que se indica el primer paso por cero, cuando $\tau=409$.

\subsubsection{Información mutua}

En cualquier caso la función de autocorrelación solo atiende a relaciones lineales entre las componentes de un vector de estado, cuando en realidad la mayoría de los sistemas físicos, como los sistemas fisiológicos, son no lineales, por lo que la función de autocorrelación podría arrojar resultados poco convincentes [Abarbanel et al., 1993]. En este sentido la información mutua constituye una mejor opción, dado que la información mutua evalúa el grado de correlación entre las componentes de un vector de estado sobre una base probabilística (no lineal), lo que permite examinar 
en qué medida dos componentes $x(t)$ y $x(t+\tau)$ están relacionadas en un contexto más general, no tan restringido al usual ámbito lineal [Fraser and Swinney, 1986]. Normalmente se sugiere el primer mínimo de la información mutua $I(\tau)$ como el valor óptimo de $\tau$ (ver figura 3.6), si bien en muchos casos, dependiendo de la resolución temporal $\Delta t$, ni siquiera puede identificarse claramente un mínimo, como sucede en los mapas logístico y de Hénon [Abarbanel et al., 1993]. La información mutua $I(\tau)$ se define como

$$
I(\tau)=\sum_{N_{r}} P[x(t), x(t+\tau)] \log _{2} \frac{P[x(t), x(t+\tau)]}{P[x(t)] P[x(t+\tau)]}
$$

donde $N_{r}$ identifica las rutas presentes en el espacio de estados. La información mutua es monótonamente decreciente con $\tau$. Conforme aumenta $\tau$, disminuye la correlación entre las componentes del vector de estado hasta un punto en el que la información mutua no sigue decreciendo o bien se hace cero, máxima decorrelación. Si se extiende el concepto de la información mutua a más componentes, esto es, si se examina la redundancia [Fraser, 1989a], el método se vuelve más sofisticado, pero requiere muchísimos más datos, no siempre disponibles, para una estimación coherente de su valor.

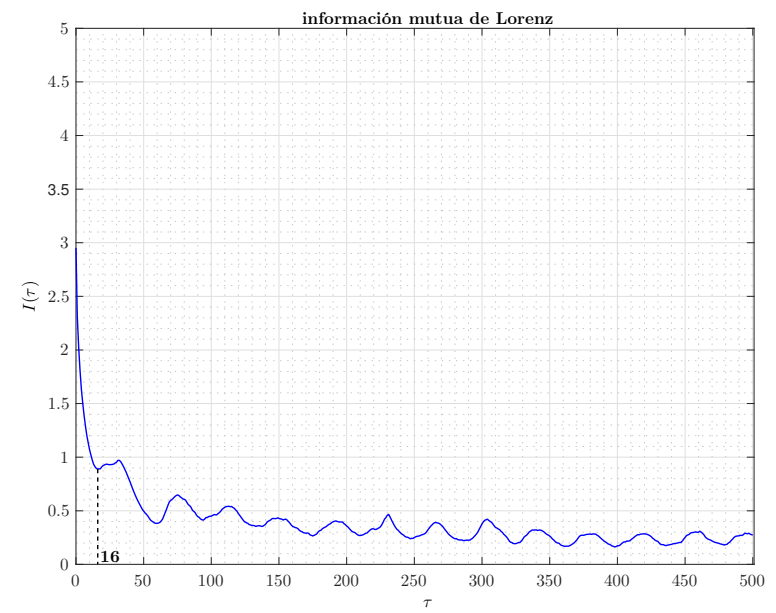

Figura 3.6: Información mutua del atractor de Lorenz para la variable dinámica $x$, en la que se indica el primer mínimo, cuando $\tau=16$.

En conexión con la información mutua, otro procedimiento para estimar el valor de $\tau$ recurre a la integral de correlación, ${ }^{3}$ dado que de por sí la propia integral de correlación es una medida de la información mutua. La integral de correlación se computa muy fácilmente, y puede adoptarse su primer mínimo como un valor adecuado de $\tau$ [Liebert and Schuster, 1989]. En el rango espacial explorado por los datos disponibles, se crea un histograma de resolución $\varepsilon$, a fin de establecer la distribución de probabilidad de los datos. Si $p_{i}$ denota la probabilidad de que una observación $x(t)$ elegida al azar esté contenida en el $i$-ésimo intervalo, de tamaño

\footnotetext{
${ }^{3}$ En adelante, dado que las sucesiones temporales son finitas, se operará con la suma de correlación, un estimador numérico de la integral de correlación.
} 
$\varepsilon$, del histograma, y $p_{i, j}(\tau)$ la probabilidad de que $x(t)$ esté contenida en el $i$-ésimo intervalo y $x(t+\tau)$ en el $j$-ésimo intervalo, la información mutua puede reescribirse como

$$
I_{\varepsilon}(\tau)=\sum_{i, j} p_{i, j}(\tau) \log _{2} p_{i, j}(\tau)-2 \sum_{i} p_{i} \log _{2} p_{i}
$$

De la ecuación (3.19) lo provechoso no es tanto el valor concreto de $I_{\varepsilon}(\tau)$ como sí su dependencia con $\tau$. El primer mínimo de $I_{\varepsilon}(\tau)$ indica el valor de $\tau$ que reduce al mínimo la redundancia entre $x(t)$ y $x(t+\tau)$. Si el primer mínimo de la información mutua difiere significativamente del primer paso por cero de la función de autocorrelación, debe ajustarse el valor de $\tau$ sobre una base no lineal dentro del rango que definen ambos extremos. Una forma de hacerlo conduce directamente a la suma de correlación, pues se ha demostrado su paralelismo con la información mutua en términos de dependencia de $\tau$ [Prichard and Theiler, 1995]. De esta manera, se computa la suma de correlación $C_{q=2}(m, \varepsilon, \tau)$, para $m=2$ y un valor de $\varepsilon$ fijo, correspondiente a la resolución del histograma, en función del parámetro $\tau$, o, lo que es lo mismo, se calcula $C_{2}(2, \varepsilon, \tau)$. Así, puede establecerse que

$$
I_{\varepsilon}(\tau)=\log _{2} C_{2}(2, \varepsilon, \tau)-2 \log _{2} C_{2}(1, \varepsilon)
$$

El rango de valores de $\tau$, según la ecuación (3.20), en el que se revela un escalamiento máximo de $C_{2}(2, \varepsilon, \tau)$ permite afinar qué valores de $\tau$ son más acertados en una potencial reconstrucción del espacio de estados.

A tenor de las diferentes alternativas para seleccionar el valor de $\tau$, no es posible decantarse por un método óptimo, pues en el proceso de selección intervienen múltiples factores colaterales [Grassberger et al., 1991] y en todos ellos es notable la ausencia de rigor analítico en la definición de un «valor óptimo de $\tau$ ». Aparte de la mayor o menor carga computacional que puedan demandar las diferentes técnicas algorítmicas, la determinación del valor óptimo de $\tau$ se supedita al esfuerzo que se está dispuesto a invertir con objeto de obtener el menor valor de $\tau$ que satisfaga los requerimientos perseguidos, según el propósito del análisis, pues a veces un valor de $\tau$ propicio para una buena reconstrucción del espacio de estados proporciona rendimientos muy pobres en un análisis predictivo. En un escenario ideal, con un número ilimitado de datos de precisión infinita y libres de ruido, es posible estimar un valor óptimo de $\tau$ con el que pueda reconstruirse fielmente el atractor original. En un experimento real esta pretensión es una utopía, y lo más oportuno reclama contrastar los resultados recabados de diferentes técnicas por medio de algún análisis discriminante. En todo caso siempre es conveniente verificar visualmente si el valor finalmente escogido de $\tau$ se antoja razonable, una vez probadas conjuntamente diversas técnicas. Eso sí, los resultados no debieran ser muy dispares, en orden de magnitud, pues esta circunstancia significaría que no se está operando con un auténtico atractor, es decir, la variación o sensibilidad que pudiera experimentar $\tau$ a las diferentes técnicas infringiría la cualidad invariante de las propiedades del atractor ante transformaciones continuamente diferenciables [Kantz and Schreiber, 2003]. 


\subsubsection{Selección del parámetro $m$. Dimensión de reconstrucción}

Una vez determinado el valor más adecuado de $\tau$, a fin de ultimar el proceso de reconstrucción del espacio de estados, resta precisar el valor mínimo de $m$ que posibilita una caracterización congruente del sistema dinámico bajo estudio. Al igual que sucede con la selección del parámetro $\tau$ no existe un único método que permita evaluar fielmente el valor de $m$ para todos los casos. En principio, conforme al teorema de Takens, el valor de $m$ que asegura una correcta reconstrucción del espacio de estados, en presencia de infinitos datos libres de ruido y de precisión infinita, obedece a $m \geqslant 2\left[d_{F}\right]+1$, siendo $d_{F}$ la dimensión fractal del atractor en que se desenvuelve la dinámica del sistema físico. Con este valor de $m$ puede establecerse con total garantía una correspondencia uno a uno entre los vectores de estado reconstruidos y los estados del sistema dinámico original.

Sin embargo, en la práctica suele disponerse únicamente de una sucesión temporal escalar, y, por consiguiente, se desconoce de antemano la dimensión fractal del sistema físico observado. Desde un punto de vista pragmático es conveniente adoptar el valor mínimo de $m$ que ofrezca ciertas garantías en términos de significación dinámica de la reconstrucción, dado que se pretende explotar el determinismo de los datos con la menor carga computacional, esto es, si se admite $m$ como la dimensión de reconstrucción óptima, para todo valor de $m^{\prime}>m$ la reconstrucción es igualmente válida, si bien valores muy grandes de $m^{\prime}$ degradan el rendimiento algorítmico de un análisis no lineal, como el cálculo de las dimensiones, las entropías, los exponentes de Lyapunov, o, incluso, un análisis predictivo, puesto que en cualquier vector de estado la diferencia temporal entre su primera componente y la última sería tan grande que la escasa relación causal entre ellas introduciría errores de estimación en los propios algoritmos.

\subsubsection{Análisis de componentes principales}

Una primera aproximación a la estimación del parámetro $m$ deriva del análisis de componentes principales (PCA, de sus siglas en inglés Principal Component Analysis), una aplicación del álgebra lineal. El método permite extraer información relevante de datos aparentemente complejos, reduciendo a priori el número de dimensiones necesarias para caracterizar la dinámica presente en los datos, esto es, permite identificar la base que mejor representa una colección de datos con ruido, detectando aquellas dimensiones redundantes que registran la misma información dinámica [Broomhead and King, 1986]. Esta base, en cierta medida, filtra el ruido y facilita la recuperación de la dinámica oculta en los datos. En suma esta composición lineal tiene por objetivo encontrar el más pequeño subespacio (hiperplano) que aproximadamente alberga el atractor.

El método asume una dimensión de reconstrucción inicial $m$ muy superior a la que supuestamente sugiere el teorema de Takens; en el caso del atractor de Lorenz, adoptado como ejemplo ilustrativo, se asume una dimensión de reconstrucción inicial de $m=9$, como puede comprobarse en la figura 3.7, si bien con una dimensión de reconstrucción más baja se obtienen resultados similares. En este sentido la intuición del experimentador juega un papel importante a la hora de decidirse por un valor inicial mayor o menor de $m$. A partir de la sucesión temporal escalar $\{x(k)\}_{k=0}^{N-1}$ ad- 
quirida, y con el valor de $\tau$ propuesto, se construyen $N-(m-1) \tau$ vectores de estado, en los que cada componente del vector simboliza una presunta variable dinámica del sistema físico. La matriz de covarianza examina la redundancia presente en las diferentes variables dinámicas que conforman el sistema dinámico. De la misma manera que la varianza mide el grado de dispersión de los valores que puede adoptar una simple variable, la covarianza mide el grado de variación conjunta de dos variables cualesquiera. Así, se define una matriz $\mathbf{X}$ de orden $m \times n$,

$$
\begin{aligned}
\mathbf{X} & =\left(\begin{array}{lcccc}
\mathbf{x}_{1} & \mathbf{x}_{2} & \cdots & \mathbf{x}_{N-(m-1) \tau}
\end{array}\right) \\
& =\left(\begin{array}{cccc}
x(1) & x(2) & \cdots & x(N-(m-1) \tau) \\
x(1+\tau) & x(2+\tau) & \cdots & x(N-(m-1) \tau+\tau) \\
\vdots & \vdots & \vdots & \vdots \\
x(1+(m-1) \tau) & x(2+(m-1) \tau) & \cdots & x(N)
\end{array}\right),
\end{aligned}
$$

donde los diferentes $\mathbf{x}_{i}$ corresponden a los vectores de estado reconstruidos, $m$ representa el número de supuestas variables dinámicas, y $n$ el número de observaciones o mediciones adquiridas de cada variable. Cada columna de $\mathbf{X}$ encarna un ensayo del experimento, en el que se capturan $m$ observaciones de $m$ diferentes variables dinámicas. Supuestamente la independencia de las diferentes variables viene impuesta por el criterio de selección de $\tau$. La matriz de covarianza $\mathbf{S}_{\mathbf{X}}$ obedece a

$$
\mathbf{S}_{\mathbf{X}}=\operatorname{cov}\left(\mathbf{X}, \mathbf{X}^{T}\right)=\frac{1}{N-(m-1) \tau} \mathbf{X X}^{T} .
$$

La entrada $S_{i j}$, para $i \neq j$, identifica la covarianza entre la $i$-ésima componente y la $j$-ésima componente de cada vector de estado. Un valor grande de una entrada $S_{i j}$, para $i \neq j$, se interpreta como una correlación significativa entre ambas componentes, a saber, la redundancia en ambas componentes es manifiesta. Con solo una de las dos componentes se dispondría de idéntica información dinámica. En caso de que $i=j$, la entrada $S_{i j}$ determina la varianza de la $i$-ésima componente de cada vector de estado. Por tanto, $\mathbf{S}_{\mathbf{X}}$ cuantifica la correlación existente entre todos los posibles pares de componentes de cada vector de estado.

En la práctica el análisis de componentes principales se computa por medio de la descomposición en valores singulares (SVD, de sus siglas en inglés Singular Value Descomposition), básicamente porque la implementación de esta descomposición es muy común en infinidad de paquetes software. Atendiendo a la descomposición en valores singulares, cualquier matriz $\mathbf{X}$ puede descomponerse en tres matrices, esto es,

$$
\mathbf{X}=\mathbf{U} \boldsymbol{\Sigma} \mathbf{V}^{T},
$$

donde $\mathbf{U}$ identifica una matriz ortogonal que caracteriza una rotación, $\boldsymbol{\Sigma}$ una matriz diagonal que señala una dilación, cuyos elementos se denominan valores singulares, y $\mathbf{V}^{T}$ identifica otra matriz ortogonal que caracteriza una nueva rotación. A fin de enlazar el análisis de componentes principales y la descomposión en valores singulares, se define, a partir de la matriz de vectores de estado $\mathbf{X}$, una matriz $\mathbf{Y}$ como 


$$
\mathbf{Y}=\frac{1}{\sqrt{N-(m-1) \tau}} \mathbf{X}^{T},
$$

donde cada columna de $\mathbf{Y}$ ha de presentar una media nula. De esta forma,

$$
\begin{aligned}
\mathbf{Y}^{T} \mathbf{Y} & =\left(\frac{1}{\sqrt{N-(m-1) \tau}} \mathbf{X}^{T}\right)^{T}\left(\frac{1}{\sqrt{N-(m-1) \tau}} \mathbf{X}^{T}\right) \\
& =\frac{1}{N-(m-1) \tau} \mathbf{X}^{T T} \mathbf{X}^{T} \\
& =\frac{1}{N-(m-1) \tau} \mathbf{X X}^{T} \\
& =\mathbf{S}_{\mathbf{X}} .
\end{aligned}
$$

De la ecuación (3.25) se deduce que $\mathbf{Y}^{T} \mathbf{Y}=\mathbf{S}_{\mathbf{X}}$, luego las componentes principales de $\mathbf{X}$ se corresponden con los autovectores de $\mathbf{S}_{\mathbf{X}}$ y los elementos de la diagonal de $\boldsymbol{\Sigma}$ con las varianzas de los correspondientes autovectores. Si se computa la descomposición en valores singulares de $\mathbf{Y}$, las columnas de la matriz $\mathbf{V}$ (ver ecuación (3.23)) identifican los autovectores de $\mathbf{Y}^{T} \mathbf{Y}=\mathbf{S}_{\mathbf{X}}$.

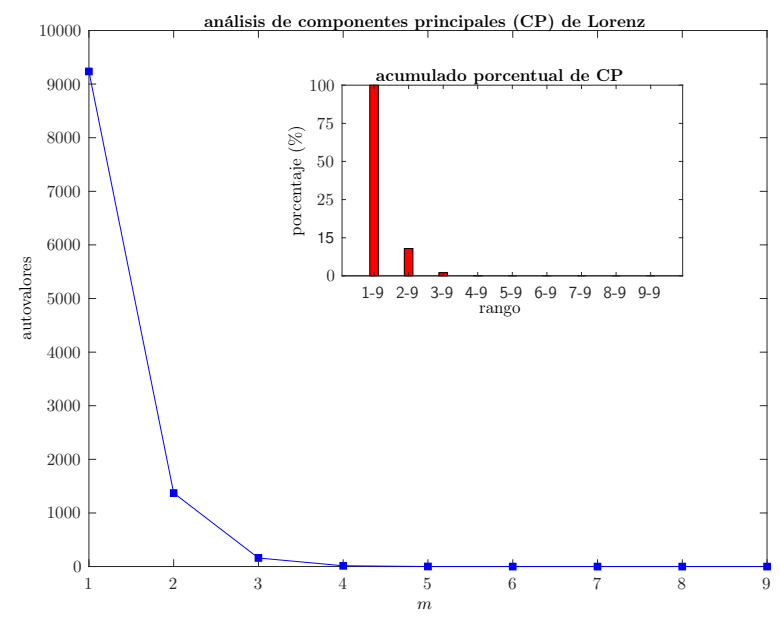

Figura 3.7: Análisis de componentes principales del atractor de Lorenz para la variable dinámica $x$; en el recuadro interior se aprecia claramente cómo con solo tres direcciones puede caracterizarse el comportamiento dinámico del atractor.

Por consiguiente, los autovectores de $\mathbf{V}$ asociados a los autovalores más grandes se convierten en las direcciones del espacio de reconstrucción que muestran mayores variaciones (varianzas) en los datos (ver figura 3.7), prescindiéndose de aquellas otras direcciones en las que las pequeñas variaciones quizás provengan de fluctuaciones propias del ruido acoplado a los datos. El hecho de desechar aquellas direcciones en principio menos importantes, aparte de reducir el efecto del ruido, especialmente el ruido blanco, facilita la aparición de una dinámica más simplificada a partir de un espacio de dimensión muy alta, de ahí que este método posibilite la reducción 
de dimensiones. En cierto modo el método del análisis de componentes principales maximiza la distancia cuadrática media entre los puntos que conforman el nuevo espacio de reconstrucción. Incluso con muy pocos datos disponibles resulta todavía factible obtener la dimensión de reconstrucción $m$ sobre una base local, en vez de global, a partir de la aproximación de la dimensión del espacio tangente en la vecindad de un único punto del espacio de vectores de estado, conforme al procedimiento de descomposición en valores singulares [Broomhead et al., 1987].

La principal desventaja de este método radica en el carácter subjetivo de la determinación del valor de $m$, pues con datos reales, o incluso con una desacertada elección inicial de $\tau$ y $m$, las diferencias entre las varianzas de las diferentes dimensiones no son siempre tan evidentes y se ha de optar por definir un umbral un tanto arbitrario que clasifique las dimensiones como principales o secundarias. Además, no existen garantías que la reconstrucción sea siempre óptima, pues el método de reconstrucción descansa sobre un análisis no paramétrico, ${ }^{4}$ y a veces es incapaz de distinguir entre un señal caótica y el propio ruido cuando presentan un espectro de potencia similar [Fraser, 1989b].

\subsubsection{Método de los falsos vecinos más cercanos}

Otro método alternativo para estimar el valor de $m$ evalúa los cambios que experimenta la estructura geométrica de un atractor a medida que se incrementa la dimensión de reconstrucción. En cualquier disposición geométrica los vectores de estado pueden ser vecinos legítimos, debido a la propia dinámica del sistema, ${ }^{5}$ o falsos vecinos, esto es, para una dimensión determinada, aparentan mantener una relación de vecindad, a nivel espacial, cuando en realidad, en su auténtica dimensión, son vecinos remotos. Si el valor de $m$ es demasiado pequeño, se evidencian bastantes autointersecciones, esto es, se muestran muchos falsos vecinos. Si el valor de $m$ es mucho mayor, las autointersecciones desaparecen porque las relaciones de falsa vecindad, propia de los falsos vecinos, son desenmascaradas. Por tanto, el objetivo de este método consiste en identificar el menor valor de $m$ que cancela la aparición de falsos vecinos (ver figura 3.8). Es el método de los falsos vecinos más cercanos (false nearest neighbours) después de que sus creadores sentaran las bases de su implementación algorítmica [Liebert et al., 1991, Kennel et al., 1992, Kennel and Abarbanel, 2002].

La idea que subyace al método es buscar puntos en la sucesión temporal que son vecinos en un espacio de reconstrucción determinado, pero que no deberían serlo pues su evolución temporal futura es muy dispar. Si, por ejemplo, se presupone que la dimensión de reconstrucción verdadera es $m$, y se establece una dimensión de reconstrucción $m^{\prime}<m$, lo que se está introduciendo es una proyección de $m$ en un nuevo sistema de coordenadas $m^{\prime}$. Debido a que con la proyección algunas coordenadas de $m$ se descartan directamente, pudiera suceder que aquellos vectores de

\footnotetext{
${ }^{4} \mathrm{Si}$ se desea incorporar el conocimiento previo que pudiera atesorarse sobre la dinámica del sistema ha de recurrirse a algún algoritmo paramétrico, que transforme los datos de acuerdo con ese conocimiento; es el denominado kernel PCA.

${ }^{5}$ Desde una perspectiva dinámica se asume que los estados evolucionan conforme a una trayectoria continuamente diferenciable, es decir, dado que estados vecinos se rigen por una casi idéntica evolución temporal, transcurrido un tiempo infinitesimal las trayectorias que manan de estados vecinos siguen conservando la relación de vecindad.
} 
estado que en el espacio $m$-dimensional difieren significativamente en las componentes que se eliminan con la proyección figuren como vecinos espaciales en el espacio $m^{\prime}$-dimensional, o sea, figuren como falsos vecinos.

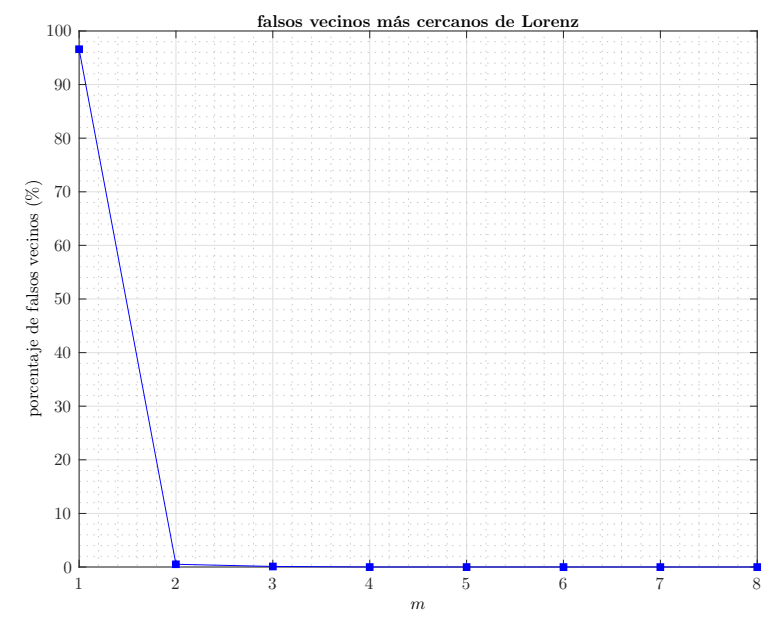

Figura 3.8: Falsos vecinos más cercanos del atractor de Lorenz para la variable dinámica $x$. A partir de $m=3$ el número de falsos vecinos más cercanos se reduce a cero.

Con todo lo antedicho, y a partir de una sucesión temporal $\{x(k)\}_{k=0}^{N-1}$ de $N$ datos escalares, se construyen, de acuerdo con un valor de $\tau$ prefijado, supuestamente óptimo, los vectores de estado $\mathbf{x}_{n}^{(m)}=\{x(n), x(n+\tau), \ldots, x(n+(m-1) \tau)\}$, respecto a un espacio $m$-dimensional. Para cada vector de estado $\mathbf{x}_{n}^{(m)}$ se computa su vecino más cercano $\mathbf{x}_{k(n)}^{(m)}$, donde $k(n)$ identifica el índice en la sucesión temporal de vectores de estado que atiende a

$$
k(n):=\left\{k \in\{0, \ldots, N-1-(m-1) \tau\}: k \neq n \wedge\left\|\mathbf{x}_{n}^{(m)}-\mathbf{x}_{k}^{(m)}\right\|=\text { mínimo }\right\},
$$

donde $\|\cdot\|$ simboliza la norma máxima, puesto que computacionalmente es más rápida que la norma euclidiana y presenta mejores prestaciones en términos de convergencia asintótica [Grassberger et al., 1991].

Si la desviación típica de la sucesión temporal asciende a $\sigma$, el estadístico que reúne numéricamente este escenario es la ratio $R_{\mathrm{fv}}$, esto es,

$$
R_{\mathrm{fv}}(r)=\frac{\sum_{n=0}^{N-1-(m-1) \tau} \Theta\left(\frac{\left\|\mathbf{x}_{n}^{(m+1)}-\mathbf{x}_{k(n)}^{(m+1)}\right\|}{\left\|\mathbf{x}_{n}^{(m)}-\mathbf{x}_{k(n)}^{(m)}\right\|}-r\right) \Theta\left(\frac{\sigma}{r}-\left\|\mathbf{x}_{n}^{(m)}-\mathbf{x}_{k(n)}^{(m)}\right\|\right)}{\sum_{n=0}^{N-1-(m-1) \tau} \Theta\left(\frac{\sigma}{r}-\left\|\mathbf{x}_{n}^{(m)}-\mathbf{x}_{k(n)}^{(m)}\right\|\right)},
$$

donde $\Theta$ denota la función escalón de Heaviside, es decir, $\Theta(x)=1$, si $x \geqslant 0$, y $\Theta(x)=$ 0 , si $x<0$, y $r$ denota el umbral que determina cuándo considerar dos vectores de estado falsos vecinos. Si la ratio entre las distancias de los vecinos más cercanos en el espacio $(m+1)$-dimensional y las distancias de los vecinos más cercanos en el 
espacio $m$-dimensional es mayor que $r$, los vectores de estado se etiquetan como falsos vecinos. El primer término del numerador de la ecuación (3.27) contabiliza el número de falsos vecinos cuando la distancia entre los vecinos más cercanos se incrementa por un factor superior a $r$ al aumentar la dimensión del espacio en una unidad. El segundo término de la ecuación descarta todos aquellos pares de vecinos más cercanos cuyas distancias son inicialmente superiores a $\sigma / r$, dado que por definición no pueden considerarse falsos vecinos vectores de estado separados espacialmente más allá de $\sigma$. Por consiguiente, son invalidados y no intervienen tanto en el numerador como en el término de normalización.

El ruido observacional afecta adversamente al método, originando falsos vecinos espurios en toda dimensión de reconstrucción $m$. En este sentido, si se pretende establecer un discriminante estadístico entre un sistema caótico y el ruido mismo, se recomienda someter los datos a un test de datos sustitutos (surrogate data), según se bosqueja en la página 70, porque incluso la elección de $\tau$ modifica el comportamiento de $R_{\mathrm{fv}}(r)$. Obviamente, con más datos disponibles en la sucesión temporal escalar la recurrencia en el atractor es más manifiesta, lo que se traduce en que las distancias entre los vecinos más cercanos son aún más pequeñas y mejora la significación estadística del algoritmo. No obstante, al incrementarse la dimensión del espacio de reconstrucción, las nuevas componentes de los vectores de estado conservan la relación señal a ruido inicial y, de alguna manera, no se ve alterada la presencia de falsos vecinos espurios. La discretización de los datos también impacta negativamente en el algoritmo, introduciendo fluctuaciones en las ratios para cualquier valor de $r$ [Kantz and Schreiber, 2003]. En definitiva el ruido puede sobreestimar la dimensión de reconstrucción obtenida [Kennel et al., 1992], y, por tanto, han de interpretarse los resultados recabados con cierta reserva. Una variante geométrica relacionada con las distancias entre pares de vecinos considera la dirección tangencial de la trayectoria trazada por los vectores de estado [Kaplan and Glass, 1992].

\subsubsection{La suma de correlación. Dimensión de correlación}

Finalmente, un tercer método muy extendido en la determinación de la dimensión de reconstrucción $m$ recurre a la suma de correlación. Diferentes estrategias de identificación y predicción incorporan la especificación de la dimensión de reconstrucción como parte de su formulación, si bien estos procedimientos van más allá del alcance de esta memoria.

Originariamente, el algoritmo de correlación tenía por objetivo cuantificar la naturaleza autosemejante de fractales de baja dimensión camuflados en sucesiones temporales [Kantz and Schreiber, 1995]. Los atractores caóticos son siempre fractales, esto es, conjuntos caracterizados por una dimensión no entera. A fin de generalizar la dimensión entera, propia de las variedades continuamente diferenciables, a conjuntos fractales se recurre a su carácter autosemejante, es decir, a la invariancia que experimentan ciertas magnitudes estadísticas con la escala [Grassberger, 1985]. Las particularidades de la invariancia (autosemejanza) determinan en cierto sentido la complejidad intrínseca de un atractor extraño y quedan resumidas en las dimensiones de Rényi de orden $q$. Si se cubre el atractor con una partición $\mathcal{P}_{\varepsilon}$ de diámetro $\varepsilon$, siendo $p_{i}$ la probabilidad de que una trayectoria del sistema dinámico frecuente el 
$i$-ésimo elemento de la partición, ${ }^{6}$ la dimensión de orden $q$ del atractor corresponde a

$$
D_{q}=\lim _{\varepsilon \rightarrow 0} \frac{1}{q-1} \frac{\ln \sum_{i \in \mathcal{P}_{\varepsilon}} p_{i}^{q}}{\ln \varepsilon}
$$

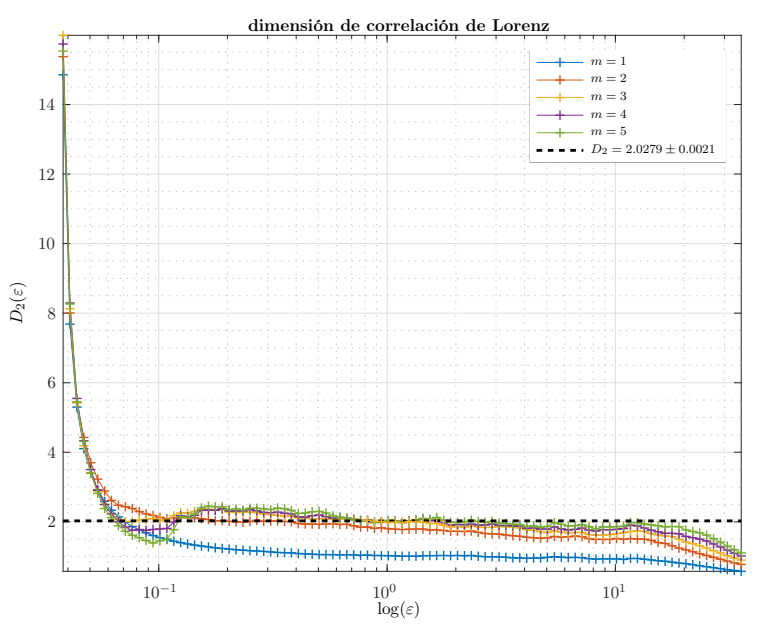

Figura 3.9: Dimensión de correlación del atractor de Lorenz para la variable dinámica $x$. A partir de $m=3$ la dimensión de correlación se confina a un valor constante en un rango intermedio y reducido de escalas $\varepsilon$.

Para el caso concreto de $q=2$, con arreglo a la ecuación (3.28), se obtiene la dimensión de correlación. El algoritmo más extendido en el cómputo de la dimensión de correlación, fundamentalmente por su facilidad de implementación y por su extrapolación inmediata a $q \neq 2$, se recoge en [Grassberger and Procaccia, 1983]. El núcleo del algoritmo reside en el cómputo de la suma de correlación $C_{2}(m, N, \varepsilon)$, una aproximación numérica de la integral de correlación, como ya se adelantó en 3.2.1.3,

$$
C_{2}(m, N, \varepsilon)=\frac{2}{(N-m)(N-m-1)} \sum_{i=m+1}^{N} \sum_{j=i+1}^{N} \Theta\left(\varepsilon-\left|\mathbf{x}_{i}-\mathbf{x}_{j}\right|\right),
$$

donde $m$ identifica la dimensión de reconstrucción, y $\Theta$ la función escalón de Heaviside, a saber, $\Theta(x)=1$, si $x \geqslant 0$, y $\Theta(x)=0$, si $x<0$. La suma de correlación $C_{2}(m, N, \varepsilon)$, conforme a la ecuación (3.29), especifica la proporción de pares de puntos del atractor localizados a una distancia menor que $\varepsilon$. Por tanto, la dimensión de correlación $D_{2}$ registra el escalamiento que experimenta $C_{2}(m, N, \varepsilon)$ con la escala $\varepsilon$, es decir, $C_{2}(m, N, \varepsilon) \propto \varepsilon^{D_{2}}$, y su valor numérico asciende a

$$
D_{2}=\lim _{\substack{N \rightarrow \infty \\ \varepsilon \rightarrow 0}} \frac{\partial \ln C_{2}(m, N, \varepsilon)}{\partial \ln \varepsilon} .
$$

\footnotetext{
${ }^{6}$ Una partición $\mathcal{P}_{\varepsilon}$ de diámetro $\varepsilon$ está compuesta por una colección contable de subconjuntos disjuntos de diámetro $\leqslant \varepsilon$. La probabilidad $p_{i}=n_{i} / N$, donde $n_{i}$ representa el número de puntos contenidos en el $i$-ésimo elemento de la partición, y $N$ el número total de puntos de la sucesión temporal.
} 
En el límite asintótico impuesto por definición en la ecuación (3.30) la dimensión $D_{2}$ alcanza un valor aproximadamente constante para un rango intermedio de escalas $\varepsilon$, como refleja la figura 3.9, siempre que la dimensión de reconstrucción $m$ sea lo suficientemente grande. En la práctica el número de puntos $N$ es finito, por lo que el escalamiento de $D_{2}$ no es visible a pequeñas escalas, debido a errores estadísticos, aun descartando, en el cómputo de $C_{2}(m, N, \varepsilon)$, aquellos pares de puntos en los que $i=j$ [Grassberger, 1988]. A pequeñas escalas se dispone de un número insuficiente de pares de puntos como para dotar de significación estadística al valor de $C_{2}(m, N, \varepsilon)$ estimado. A nivel algorítmico, el tiempo de cómputo es del orden de $O\left(N^{2}\right)$, si bien se han ideado diferentes estrategias de búsqueda de pares de puntos cercanos que, además de organizar los datos de forma eficiente, evitan tener que examinar toda la secuencia de datos sin incurrir en errores sistemáticos o estadísticos, lo que ha permitido reducir la carga computacional [Schreiber, 1995].

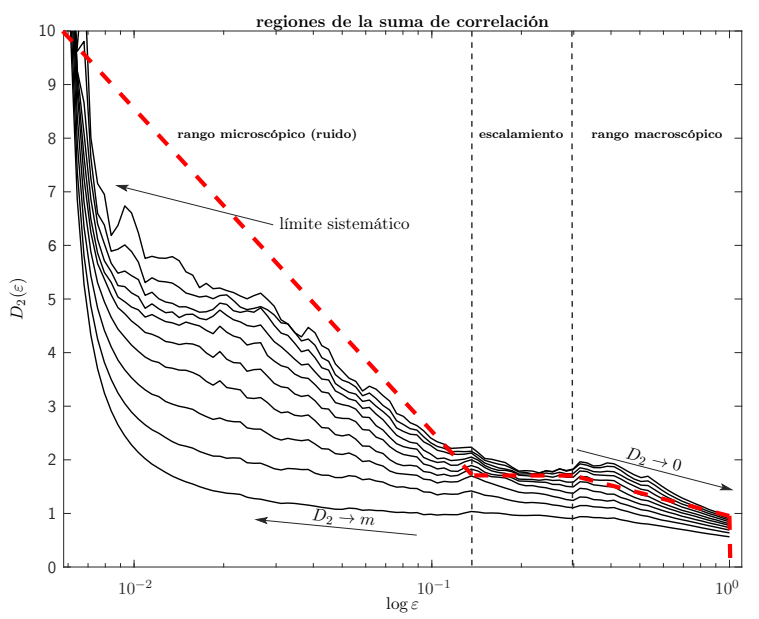

Figura 3.10: Regiones características de la suma de correlación.

En una representación gráfica típica de la suma de correlación, $\ln C_{2}(m, N, \varepsilon)$ vs. $\ln \varepsilon$, de una sucesión temporal adquirida de un sistema físico real, pueden observarse diferentes regiones (ver figura 3.10):

1. Rango de escalas macroscópicas. Por encima de un valor de $\varepsilon$, según datos experimentales, $\varepsilon \geqslant(1 / 5) L$, siendo $L$ la extensión del atractor, el atractor se reduce a un número finito de puntos que ocupan un espacio muy delimitado. La estructura macroscópica prevalece en detrimento de un posible escalamiento que revele la naturaleza fractal del atractor. La dimensión de correlación tiende a cero, $D_{2} \rightarrow 0$.

2. Rango de escalamiento. En un rango intermedio de escalas es posible distinguir el verdadero escalamiento que experimenta la suma de correlación con la escala para una dimensión de reconstrucción $m_{\text {mín }}>D_{2},{ }^{7}$ es decir, a par-

\footnotetext{
${ }^{7} \mathrm{El}$ algoritmo de correlación no requiere una estimación a priori de la dimensión de reconstrucción, de ahí que sobre la misma plantilla gráfica se tracen diferentes curvas para diferentes valores de $m$, en un intervalo limitado $2 \leqslant m \leqslant 15$, con objeto de impedir fluctuaciones estadísticas o sistemáticas.
} 


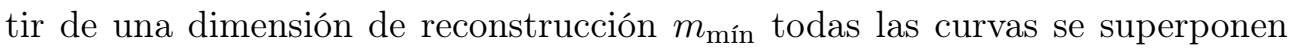
aproximadamente, los valores de la suma de correlación se circunscriben a una recta cuya pendiente alude a la dimensión de correlación.

3. Ruido. En presencia de datos contaminados con ruido, para un valor de $\varepsilon$ del orden de la amplitud del ruido, el factor dominante es la componente estocástica que introduce el ruido, lo que se traduce en que los puntos se distribuyen aleatoriamente, tendiendo a ocupar todo el espacio disponible, esto es, el espacio que, para cada curva, se asocia a la dimensión de reconstrucción $m$. El exponente de escalamiento, la dimensión de correlación, en este caso, aumenta hasta alcanzar la dimensión de reconstrucción $m$.

4. Límite sistemático. En escalas muy pequeñas, muy por debajo del rango atribuido al ruido, e incluso por debajo de la distancia media entre puntos, $N^{-1 / m}$, la carencia de pares de puntos cercanos deteriora drásticamente la estimación de la suma de correlación, lo que deriva en fluctuaciones en las curvas que desdibujan cualquier posible estructura.

A tenor de estas indicaciones, el régimen macroscópico, el ruido y el límite sistemático merman el rango de escalamiento del que poder extraer la dimensión de correlación, y, por consiguiente, merecen especial examen, con objeto de intentar reducir su efecto y así poder interpretar correctamente los resultados recabados.

A escalas $\varepsilon$ muy pequeñas, se requiere un número mínimo de pares de puntos cercanos [Smith, 1988, Eckmann and Ruelle, 1992], del orden de 100, para que la suma de correlación proporcione un valor satisfactorio y no se vea interrumpido en exceso el rango de escalamiento. Por tanto, la longitud mínima $N_{\text {mín }}$ de la sucesión temporal, a fin de que la suma de correlación pueda resolver eficazmente una dimensión de correlación $D_{2}$, o sea, que la suma de correlación pueda procurar una región de escalamiento sobre al menos una octava de escalas, obedece a

$$
N_{\text {mín }} \approx 10^{\left(D_{2}+2\right) / 2},
$$

de lo que se deduce que, para una sucesión temporal de $N$ puntos, la dimensión de correlación máxima $D_{\text {máx }}$ que podría entregar, con ciertas garantías, la suma de correlación sería de

$$
D_{\text {máx }} \approx 2 \log _{10} N-2 .
$$

En general, en el límite del rango macroscópico (cota superior del rango de escalamiento) los errores estadísticos crecen conforme aumenta la dimensión del atractor, manteniéndose fijo el número de puntos $N$. En el límite inferior, el rango de escalamiento está delimitado por el ruido, con independencia del número de puntos $N$ presentes. En este sentido puede resultar muy beneficioso introducir algún mecanismo de reducción de ruido, con objeto de extender el rango de escalamiento, si bien los mecanismos de reducción de ruido más efectivos suelen requerir muchos datos.

Con datos contaminados de ruido, el rango de escalamiento no es siquiera visible, o cuando menos indefinido, cuando este se sitúa en el radio de influencia del ruido, es decir, del orden de un múltiplo entero - el factor multiplicativo depende del tipo de 
ruido - de la amplitud del ruido. Dado que ya de por sí la estructura macroscópica del atractor condiciona por arriba el rango de escalamiento, el margen disponible para una correcta interpretación no es muy amplio y, en muchas aplicaciones, solo admite un $2 \%$ de nivel de ruido [Schreiber and Kantz, 1995].

En el contexto de las señales biológicas han de adoptarse ciertas precauciones antes de proceder con el cómputo y posterior análisis de la dimensión de correlación, debido primordialmente a la naturaleza no estacionaria de la mayoría de los fenómenos biológicos y a la presencia de estocasticidad que enmascara la presumible componente determinista del sistema fisiológico, caracterizada por un atractor de baja dimensión.

Una sucesión temporal comprende una colección finita de puntos, de ahí que en principio, por definición, su dimensión sea cero. No obstante, desde un punto de vista estadístico, puede considerarse la sucesión temporal como una muestra de una población infinita, el atractor bajo estudio. Por consiguiente, y para un rango intermedio de escalas, el algoritmo de correlación ha de estimar la sucesión temporal como una muestra estadística, ignorando en cierto sentido la finitud de los datos. Además, el algoritmo ha de aspirar a conseguir una rango de escalamiento lo más extenso posible. En este sentido ligeras modificaciones en el algoritmo logran el objetivo. Por un lado, ha de eliminarse la correlación dinámica (temporal) a la hora de evaluar la relación de cercanía entre puntos, consecuencia del sobremuestreo (oversampling) del atractor, ${ }^{8}$ que, en el mundo real, deriva de sistemas que evolucionan continuamente en el tiempo. Si se incluye la correlación dinámica, los puntos cercanos de un punto dado se reducirán a sus vecinos inmediatos sucesores o predecesores, y, por consiguiente, el algoritmo de correlación resuelve erróneamente que la dinámica del sistema se desarrolla en un espacio unidimensional. A medida que se incrementa la dimensión de reconstrucción el rango de escalamiento para el que se manifiesta una dimensión unitaria se ensancha. A fin de evitar esta confusión sistemática, el algoritmo debe descartar aquellos puntos relacionados dinámicamente, esto es, aquellos

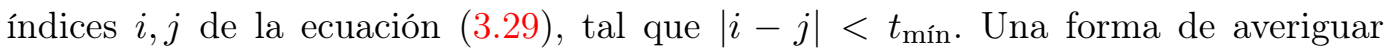
qué valor de $t_{\text {mín }}$ excluye la correlación dinámica del algoritmo recurre a la separación espacio-temporal [Provenzale et al., 1992], que, visualmente, evalúa para qué intervalo temporal la distancia de separación entre los puntos del atractor obedece únicamente al espacio físico que hay entre ellos y no a la evolución dinámica, es decir, permite diferenciar las correlaciones temporales de las correlaciones geométricas.

Muchas veces la muestra adquirida no es representativa del atractor subyacente, esto es, no es una muestra aleatoria del atractor y todas las observaciones están dinámicamente correladas. La muestra es no estacionaria, no es fiel reflejo de la medida invariante del atractor (distribución independiente del tiempo), y posiblemente reproduzca solo una parte del atractor, por lo que puede darse el caso de que las ob-

${ }^{8} \mathrm{El}$ tiempo entre observaciones ha de ser lo suficientemente pequeño como para capturar la dinámica a pequeñas escalas, a saber, los cambios repentinos (sutilezas, irregularidades, rugosidades) que experimenta el sistema, propios de atractores extraños. Sin embargo, con tantas muestras en tan corto intervalo de tiempo, se manifiesta una correlación temporal significativa, esto es, la función de autocorrelación decae lentamente, lo que significa que puntos consecutivos en el tiempo lo son también en el espacio. El caso extremo corresponde a que un punto es vecino de sí mismo, $i=j$ en la ecuación (3.29). 
servaciones aparezcan confinadas en una región del espacio de baja dimensión, aun pudiendo proceder de un atractor $\infty$-dimensional, como el propio ruido o un movimiento browniano. De este modo, el algoritmo de correlación estaría proporcionando falsamente una dimensión finita, cuando en realidad el sistema es de dimensión infinita. Una medida de la ausencia de estacionariedad se evidencia en la separación espacio-temporal, donde no se apreciaría un aplanamiento de las curvas conforme aumenta la separación temporal entre pares de puntos; otro mecanismo que delata la falta de estacionariedad en los datos es el espectro de potencia, en el que una concentración de potencia en las bajas frecuencias revela la imposibilidad de obtener un exponente de escalamiento finito, puesto que prácticamente todos los datos están correlacionados temporalmente, existe una tendencia en los datos que varía lentamente. Una forma de eliminar esta tendencia global, que impide consolidar una clara región de escalamiento, y, por tanto, conferir cierta estacionariedad a los datos, recurre a mecanismos de eliminación de la tendencia (detrending), como operar con la primera derivada de la sucesión temporal, en lugar de con los datos originales. Sin embargo, con estas transformaciones empeora la relación señal a ruido, lo que, a su vez, redunda negativamente en la apreciación de un régimen de escalamiento a pequeñas escalas. En cualquier caso se busca una región de escalamiento en la que las curvas de la suma de correlación se saturen, o, dicho de otro modo, una región de escalamiento independiente de la dimensión de reconstrucción. Obviamente, además de eliminar falsos espurios, debidos, entre otros factores, a la correlación dinámica, resulta indispensable en el cómputo de la suma de correlación escoger previamente un valor de $\tau$ adecuado, que asegure en cierto modo la independencia entre las componentes de cada vector de estado. En este aspecto se recomienda hacer pruebas con diferentes valores de $\tau$, pues, como se ha comentado previamente, el valor de $\tau$ no es único para todos los algoritmos y, en consecuencia, cada estadístico de interés reclama su propio valor de $\tau$ más efectivo.

En señales biológicas es difícil discernir una región de escalamiento patente, dado que estas señales no proceden de un sistema aislado, sino más bien de un conjunto de subsistemas interrelacionados (perturbación del entorno circundante). No obstante, con la integral de correlación todavía puede especularse una no linealidad, si acaso determinista, que no deriva simplemente de la contribución del ruido. Un método para descartar que un proceso aleatorio ha sido el mecanismo generador de los datos descansa en la noción de los datos sustitutos (surrogate data) [Theiler et al., 1992]. Conforme a un contraste de hipótesis, se especifica una hipótesis nula por la que los datos corresponden a ruido blanco o coloreado. Para ello se construye un conjunto hipotético de sucesiones temporales de test con propiedades lineales similares a las de los datos que configuran la sucesión temporal inicial. Esto significa que el espectro de potencia coincide en todos los casos. En este sentido se calcula la transformada de Fourier de los datos originales, se procede con un proceso de aleatorización de las fases de los diferentes coeficientes de Fourier, y mediante la transformada inversa se dispone de una sucesión temporal de la misma longitud que los datos originales, con idéntico espectro de potencia, pero generados, de otro modo, por un proceso aleatorio. Se computa posteriormente la suma de correlación para cada una de las sucesiones temporales de prueba y, por medio del test estadístico, se dirime cómo de probable es que los datos originales hayan sido generados por un 
proceso aleatorio. En caso de que se manifieste una diferencia significativa entre los datos originales y los datos de test, se puede afirmar, con bastante confiabilidad, que los datos podrían esconder algo más que un simple modelo lineal.

En no pocas ocasiones, aun admitiéndose cierta estacionariedad en los datos, las señales biológicas están contaminadas por ruido de gran amplitud, destruyendo su posible estructura fractal a pequeñas escalas, incluso si el atractor es de baja dimensión. No obstante, la geometría global del atractor todavía puede advertirse a gran escala, de forma que, ante un test estadístico basado en datos sustitutos, puede convertirse en una prueba irrefutable de determinismo en los datos adquiridos, a pesar de que con estos datos no pueda extraerse la verdadera dimensión del atractor.

En líneas generales las diversas fuentes de error, en el cómputo de la dimensión de correlación, inducen una estimación a la baja de su valor real, a excepción del ruido, que, aparte de destruir la estructura fractal del atractor, tiende a aumentar falsamente la dimensión de correlación suministrada por el algoritmo. En cualquier caso la estimación de la dimensión de correlación supedita su legitimidad a la estacionariedad de los datos. La ausencia de estacionariedad invalida todo valor de dimensión de correlación aportado por el algoritmo. Las presumibles fluctuaciones que puedan experimentar las curvas de la suma de correlación en la región de escalamiento, para diferentes dimensiones de reconstrucción $m$, se deben tanto a desviaciones sistemáticas como a desviaciones estadísticas, de ahí que en vez de ajustar una línea recta a la región de escalamiento, con objeto de promediar el valor de la dimensión de correlación, sea más conveniente aplicar un estimador de máxima verosimilitud [Theiler, 1988]. En este sentido la lacunaridad del objeto geométrico definido por los vectores de estado, para cada dimensión de reconstrucción $m$, puede introducir fluctuaciones en la suma de correlación que, según su amplitud, podrían desdibujar una región de escalamiento aparente. La lacunaridad, término acuñado por Benoît Mandelbrot [Mandelbrot, 1983], es una medida, dependiente de la escala, de la heterogeneidad o textura de un objeto, sea o no fractal [Plotnick et al., 1996]; la distribución espacial de los puntos viola la invariancia traslacional del objeto geométrico, esto es, a grandes escalas se muestran homogéneos, mientras que a pequeñas escalas revelan su hetereogeneidad, o viceversa.

Dada la actualidad científica del caos determinista, no es infrecuente encontrar caos en todas las señales capturadas del mundo real. En el caso de las señales biológicas, la aperiodicidad y el determinismo pueden resolverse fácilmente mediante técnicas convencionales o, inclusive, por medio del algoritmo de correlación. Sin embargo, la presencia de un atractor de baja dimensión no resulta tan evidente en la dimensión de correlación. El ruido y la longitud limitada de las sucesiones temporales disponibles enmascaran la región de escalamiento, o, mejor dicho, el determinismo aparente. Un atractor de baja dimensión es condición suficiente para que se desarrolle el caos determinista, pero no es realmente necesaria; sistemas con realimentación, como suele ocurrir en el caso de señales biológicas, obedecen a ecuaciones diferenciales con retardo, que, por definición, atienden a infinitos grados de libertad y, ciertamente, no se muestran proclives a una reconstrucción del espacio de estados. El algoritmo de reconstrucción proporciona resultados satisfactorios siempre que los datos sean estacionarios, algo inusual en señales biológicas; en caso contrario los resultados obtenidos únicamente pueden llevar a presentimientos poco concluyentes 
sobre el proceso físico responsable de la dinámica presente, y sería menester recurrir a otras herramientas de análisis, con objeto de caracterizar, con nueva información complementaria, el verdadero comportamiento que exhibe el atractor bajo estudio.

\subsection{Otros métodos de estimación de $\tau$ y $m$}

La reconstrucción del espacio de estados consiste en reproducir una sucesión temporal escalar $\{x(k \Delta t)\}_{k=1}^{N}$ en un espacio euclidiano $\mathbb{R}^{m}$, donde $m$ identifica la dimensión de reconstrucción y $\Delta t$ el tiempo de muestreo de los datos. Con este procedimiento se espera que los puntos en el espacio $\mathbb{R}^{m}$ conformen un atractor que preserve las propiedades topológicas del atractor original, a priori desconocido. Las principales técnicas de reconstrucción se basan en el método de los retardos y suelen calcular los parámetros $\tau$ y $m$ de forma empírica y, por tanto, no necesariamente proporcionan estimaciones adecuadas, aun admitiendo de por sí un alto de grado de incertidumbre cuando se opera con datos reales. En este sentido se han planteado procedimientos que pretenden mejorar las estimaciones de $\tau$ y $m$, como es el caso de la longitud de la ventana temporal, $\tau_{w}=(m-1) \tau$, con $\tau=n \Delta t$, que abarca cada vector de estado y que, en cierta medida, está relacionada con la cantidad de información transferida desde la sucesión temporal al espacio de reconstrucción. Una vez evaluado el valor de $\tau_{w}$ más idóneo, mediante la dimensión de correlación puede verificarse la calidad de la reconstrucción, dado que es probable que la idoneidad de $\tau_{w}$ para una estimación acertada de la dimensión pueda extrapolarse a otras invariantes, como la entropía, los exponentes de Lyapunov, e incluso a análisis predictivos [Kugiumtzis, 1996].

Una aproximación geométrica del procedimiento de reconstrucción estriba en considerar la reconstrucción como una proyección ortogonal de un espacio de estados $d$-dimensional en un subespacio $m$-dimensional, esto es, se define un mapa lineal

$$
\pi^{(m)}: \mathbb{R}^{d} \rightarrow \mathbb{R}^{m}
$$

que transforma cada vector $\mathbf{x}_{k}^{d}$ en un vector de estado $\mathbf{x}_{k}^{m}$. Las $d$ coordenadas de $\mathbf{x}_{k}^{d}$ incluyen todas las muestras de la ventana temporal $\tau_{w}=d-1=(m-1) n$. Las $m$ componentes del subespacio proyectado corresponden a $m$ muestras del espacio $d$ dimensional tomadas a intervalos de $n$ muestras, de ahí la importancia del parámetro global $\tau_{w}$. Dependiendo del valor de $n$ puede o no cubrirse la ventana temporal $\tau_{w}$, lo que incide directamente en la calidad de la reconstucción; por ejemplo, con un valor de $n$ pequeño, véase $n=1$, las muestras difieren muy poco, pues solo se consideran las $n$ primeras muestras de la ventana temporal. En este escenario la reconstrucción es muy pobre, ya que los vectores de estado se extienden a lo largo de la bisectriz en $\mathbb{R}^{m}$.

El parámetro $\tau$ determina el grado de correlación entre las componentes de cada vector de estado. Si el valor de $\tau$ es muy pequeño, las componentes están fuertemente correlacionadas y el atractor se dispone a lo largo de la diagonal. A medida que se incrementa $\tau$ la correlación entre las componentes va reduciéndose, el atractor se expande, apartándose de la diagonal, hasta un límite en el que el atractor se repliega y vuelve a aparecer la correlación entre las componentes. En teoría la reconstrucción 
del atractor conlleva una equivalencia topológica entre el atractor reconstruido y el atractor original. Una forma de evaluar esta correspondencia implica comprobar si los procesos de estiramiento y plegado son similares en ambos atractores, bien verificando que la distancia entre los puntos del atractor es proporcionalmente similar en los atractores reconstruido y original, o bien confrontando que puntos cercanos en el atractor original mantienen su proximidad en el atractor reconstruido. No obstante, evidencias empíricas demuestran que la expansión per se de los vectores de estado de la diagonal no garantiza una reconstrucción del espacio de estados que guarde una relación de equivalencia con el espacio de estados original. Además, no siempre las funciones de autocorrelación y de la información mutua son monótonamente decrecientes, sino que exhiben fluctuaciones, debido a comportamientos pseudoperiódicos presentes en muchos sistemas, que se traducen en correlaciones entre componentes de los vectores de estado distanciadas entre sí, esto es, si, por ejemplo, el primer mínimo de la información mutua asegura un mínimo de correlación entre componentes consecutivas de cada vector de estado, las fluctuaciones en la información mutua no aseguran que no exista correlación entre componentes no contiguas.

El parámetro $m$ resuelve el mínimo valor de la dimensión de reconstrucción que evita la presencia de autointersecciones en el atractor reconstruido. Las autointersecciones impiden que la dinámica del sistema reconstruido se desarrolle en una variedad continuamente diferenciable. Aparte de la dimensión de correlación, el método de los falsos vecinos más cercanos se ha convertido en un procedimiento muy habitual para comprobar la ausencia de autointersecciones, dado que una vez alcanzada la dimensión de reconstrucción $m$ que asegura la inexistencia de autointersecciones, todos los puntos vecinos en $\mathbb{R}^{m}$ deberían ser igualmente vecinos en $\mathbb{R}^{m+1}$. No siempre se cumple que las relaciones de vecindad se mantengan cuando se incrementa la dimensión de reconstrucción, debido a la interdependencia entre $m$ y $\tau$. El valor de $m$ que suministra el método de los falsos vecinos más cercanos depende del valor de $\tau$ escogido, y, por consiguiente, los resultados obtenidos son un tanto arbitrarios. Por otro lado, el método de los falsos vecinos más cercanos es muy sensible al ruido, máxime con valores grandes de $\tau$, lo que lleva a sobreestimar el valor de $m$ que realmente procura una reconstrucción del espacio de estados [Aleksić, 1991]. En el caso del análisis de componentes principales, no siempre se revela claramente un espacio de reconstrucción conforme a las varianzas de cada una de las direcciones. No en pocas ocasiones el atractor adopta una configuración muy parecida al ruido e intenta ocupar todas las direcciones del espacio de reconstrucción, de ahí que se recurra a un análisis local del espacio tangente, inclusive más robusto frente al ruido, a fin de apreciar más abiertamente la dimensión de reconstrucción [Passamante et al., 1989], si bien se supedita a la dimensión del hiperespacio a partir del cual se efectúan las proyecciones, es decir, su rendimiento está condicionado por el valor de $\tau_{w}$.

Con estos antecedentes, en los que es manifiesta una cierta arbitrariedad en la elección por separado de los valores de $\tau$ y $m$ que mejor reconstruyen el espacio de estados, la longitud de la ventana temporal $\tau_{w}$ puede disipar la incertidumbre. Una primera tentativa al valor de $\tau_{w}$ vincula su valor a la memoria del sistema, es decir, al número mínimo de observaciones necesarias para consolidar una predicción futura con cierta fiabilidad. Desde una perspectiva geométrica, una alternativa práctica se asienta en el período orbital medio $T_{o}$, esto es, el tiempo medio entre dos pasos 
consecutivos por la misma vecindad, que, en muchos atractores, sugiere el tiempo que transcurre entre dos intersecciones sucesivas de la sección de Poincaré (véase 5.3.2 para una explicación algo más extensa). A partir de una sucesión temporal escalar, una solución más factible, y con resultados similares, considera el tiempo entre picos $T_{p}$, las oscilaciones de la sucesión temporal, ${ }^{9}$ como una primera estimación de $\tau_{w}$. En general $T_{p}<T_{o}$, por lo que $T_{p}$ comporta una cota inferior de $\tau_{w}$, o sea, $\tau_{w}>T_{p}$. En ocasiones se opera directamente en el dominio de la frecuencia [Broomhead and King, 1986, Grassberger et al., 1991], en lugar de en el dominio del tiempo, si bien en algunos sistemas caóticos no es posible apreciar frecuencias dominantes y, por consiguiente, no pueden detectarse picos suficientemente definidos. En otros casos se ha delimitado el valor de $\tau_{w}$ a partir de marcas características de la función de autocorrelación y de la información mutua. Por ejemplo, en [Albano et al., 1988] se propone $\tau_{c} \leqslant \tau_{w} \leqslant 4 \tau_{c}$, donde $\tau_{c}$ identifica el instante de tiempo en el que la función de autocorrelación alcanza el valor 1/e; en [Gibson et al., 1992] se plantea una cota superior para $\tau_{w}$, a saber,

$$
\tau_{w} \leqslant \sqrt{\frac{3\langle x(t)\rangle}{\langle\dot{x}(t)\rangle}},
$$

donde $\langle x(t)\rangle$ denota la media de la sucesión temporal y $\langle\dot{x}(t)\rangle$ la media de la primera derivada de la sucesión temporal.

En caso de pretender evaluar el valor de $\tau_{w}$ conforme a la dimensión de correlación $D_{2}$, se suele proceder de forma heurística, mediante un mecanismo de ensayo y error en el que, para diferentes valores de $\tau$ y $m$, se efectúan comprobaciones del grado de estabilidad que experimentan los resultados en un rango de posibles valores de $\tau_{w}$. Un atributo clave es que, en sistemas caóticos, la estimación de la dimensión de correlación no sufre cambio alguno por variar $\tau$ o $m$ siempre que $\tau_{w}$ permanezca constante. Una vez determinado el valor de la longitud de la ventana de tiempo $\tau_{w}$, se dispone de la dimensión $d$ del hiperespacio, y la elección de la proyección no entraña dificultad, siempre que la proyección satisfaga los requerimientos básicos, es decir, que $m>D_{2}$ y que

$$
n \approx \frac{d-1}{m-1} \equiv \frac{\tau_{w}}{m-1},
$$

de manera que las distancias entre puntos en $\mathbb{R}^{d}$ y $\mathbb{R}^{m}$ muestren un comportamiento estadístico similar. En sucesiones temporales de corta longitud o con datos ruidosos, la estimación de la dimensión de correlación es más sensible al valor de $\tau_{w}$. El ruido y un número insuficiente de datos enmascaran la región de escalamiento, lo que incrementa la incertidumbre en la estimación de la dimensión de correlación y reduce el rango de opciones válidas de $\tau_{w}$ en el que es posible encontrar una región de escalamiento efectiva de la dimensión de correlación cuando se representa $\log C_{2}(r)$ vs. $\log r$. A medida que incrementa el ruido presente en los datos la región de escalamiento se hace más aparente conforme $\tau_{w}$ se aproxima a $T_{p}$ desde arriba. Con

\footnotetext{
${ }^{9}$ Normalmente se filtra la sucesión temporal con objeto de resaltar los picos representativos en los patrones de oscilación y eliminar los ocasionales picos espurios debidos al ruido. A los filtros de suavizado (smoothing) de orden $k$ se les atribuyen buenos rendimientos. Un filtro de suavizado captura patrones generales ocultos en los datos.
} 
pocos datos disponibles se subestima la dimensión de correlación debido al escaso número de vecinos a pequeña escala, lo que otorga apenas significación estadística al algoritmo. Con valores de $\tau_{w}$ muy superiores a $T_{p}$ es probable que se sobreestime la dimensión de correlación [Kugiumtzis, 1996]. Con este escenario es recomendable emprender diversos ensayos, a fin de refinar el valor mínimo de $m$ que mejor se adapte a los datos disponibles.

Los teoremas matemáticos que sientan las bases de la reconstrucción de un atractor demuestran la existencia de valores de $\tau$ y $m$ que posibilitan una correspondencia uno a uno entre los estados del sistema dinámico de interés y los vectores de estado, derivados de la reconstrucción mediante el método de los retardos, si bien no especifican cómo obtener los valores adecuados, de ahí que se hayan planteado en los últimos años múltiples estrategias, la mayoría de las veces heurísticas, con objeto de inferir los parámetros característicos de la reconstrucción del espacio de estados.

En [Pecora et al., 2007] se plantea un estadístico basado en las relaciones funcionales que se establecen entre las componentes de cada vector de estado reconstruido, en estrecha correspondencia con los fundamentos matemáticos que respaldan la teoría de la reconstrucción del espacio de estados. Este estadístico resuelve simultáneamente qué valores de $\tau$ y $m$ permiten la reconstrucción del espacio de estados a partir de una sucesión temporal multivariable, a la par que facilita la verificación de la calidad de la reconstrucción y la determinación de la ventana temporal de cada vector de estado, conforme a las múltiples escalas de tiempo presentes en la sucesión temporal.

A diferencia de otras alternativas metodológicas a la estimación de los parámetros de la reconstrucción del espacio de estados, en las que se requieren umbrales seleccionados de un modo un tanto arbitrario, con este estadístico únicamente se precisa un nivel de confianza en la reconstrucción del atractor, supeditado a la aplicación que promueve el análisis de los datos.

Si $\mathbf{x}_{t}=[x(t), x(t+\tau), \ldots, x(t+(m-1) \tau)]^{T}$ comprende un vector de estado reconstruido, Floris Takens [Takens, 1981] y Tim Sauer et al. [Sauer et al., 1991] reivindican como requerimiento esencial en toda reconstrucción del espacio de estados que $x(t+\tau)$ ha de ser funcionalmente independiente de $x(t)$. Para extender este requisito a una sucesión temporal multivariable, se define esta como $\left\{x_{i}(t)\right\}_{t=1}^{N}$, con $i=1, \ldots, M$, compuesta por $N$ observaciones, adquiridas a intervalos regulares, de $M$ variables. Así, se construye inicialmente un vector de estado $m^{\prime}$-dimensional, en el que cada componente del vector se escoge aleatoriamente entre las $M$ posibles variables, esto es,

$$
\mathbf{x}_{t}=\left[x_{i_{1}}\left(t+\tau_{1}\right), x_{i_{2}}\left(t+\tau_{2}\right), \ldots, x_{i_{m^{\prime}}}\left(t+\tau_{m^{\prime}}\right)\right]^{T}, \quad \text { para } i_{k}=1, \ldots, M
$$

donde, además, los retardos $\tau_{j}$ entre las componentes del vector de estado no tienen por qué coincidir. La resolución sobre añadir o no una nueva componente al vector de estado radica en la independencia funcional entre la nueva componente candidata y las componentes previas, es decir, ha de comprobarse si existe alguna función $f_{\text {test }}: \mathbb{R}^{m^{\prime}} \rightarrow \mathbb{R}$, tal que 


$$
x_{i_{m^{\prime}+1}}\left(t+\tau_{m^{\prime}+1}\right) \underset{i ?}{=} f_{\text {test }}\left(x_{i_{1}}\left(t+\tau_{1}\right), x_{i_{2}}\left(t+\tau_{2}\right), \ldots, x_{i_{m^{\prime}}}\left(t+\tau_{m^{\prime}}\right)\right) .
$$

El proceso seguirá incorporando nuevas componentes al vector de estado hasta que se agoten las opciones, en cuyo caso se da por concluido el procedimiento, proporcionándose conjuntamente los valores de los parámetros $\tau$ y $m$ que mejor reconstruyen el atractor subyacente.

El estadístico que se aplica para verificar la relación funcional entre componentes del vector de estado es la continuidad de $f_{\text {test }}$, esto es, puntos cercanos en el dominio de $f_{\text {test }}$ son mapeados a puntos cercanos en el rango de $f_{\text {test }}$. Por tanto, conforme a la ecuación (3.37), se elige un punto de referencia $x_{i_{m^{\prime}+1}}\left(t_{\text {ref }}+\tau\right) \in \mathbb{R}$, con un valor de $\tau$ fijo, y se define una bola de radio $\varepsilon$ alrededor del punto. Igualmente, se define una bola de radio $\delta$ alrededor del punto de referencia $\mathbf{x}_{t_{\mathrm{ref}}}=\left[x_{i_{1}}\left(t_{\mathrm{ref}}+\tau_{1}\right), x_{i_{2}}\left(t_{\mathrm{ref}}+\right.\right.$ $\left.\left.\tau_{2}\right), \ldots, x_{i_{m^{\prime}}}\left(t_{\mathrm{ref}}+\tau_{m^{\prime}}\right)\right]^{T} \in \mathbb{R}^{m^{\prime}}$, asociado al punto $x_{i_{m^{\prime}+1}}\left(t_{\mathrm{ref}}+\tau\right)$. Suponiendo que la bola de radio $\delta$ contuviera $u$ puntos y $v$ puntos fueran mapeados por la función $f_{\text {test }}$ a la bola de radio $\varepsilon$, se recurre al contraste de hipótesis (hipótesis nula), por el que se enuncia que los $v$ puntos fueron mapeados aleatoriamente, con probabilidad $p$, a la bola de radio $\varepsilon$, para corroborar o no la continuidad de la función $f_{\text {test }}$. Normalmente $p=1 / 2$, dado que es más difícil de rechazar y exhibe robustez frente al ruido aditivo [Pecora et al., 1995]. De la misma manera, ha de seleccionarse un nivel de confianza $\alpha$ para el que se rechaza la hipótesis nula. Si la probabilidad de que $v$ o más puntos, de los $u$ puntos presentes en la bola de radio $\delta$ (distribución binomial), son mapeados a la bola de radio $\varepsilon$ es menor que $\alpha$, se rechaza la hipótesis nula, o, lo que es lo mismo, se constata la continuidad de la función $f_{\text {test }}$. El proceso se repite reduciendo $\varepsilon$ y variando $\delta$ hasta que no pueda rechazarse la hipótesis nula. El valor más pequeño de $\varepsilon$ alcanzado se convierte en el estadístico de continuidad, $\varepsilon_{\text {cont }}$, si bien suele emplearse un valor promedio $\left\langle\varepsilon_{\text {cont }}\right\rangle$, de acuerdo con los valores de $\varepsilon_{\text {cont }}$ recabados de los diferentes puntos de referencia que se utilicen en el test. Cuanto más grande es $\left\langle\varepsilon_{\text {cont }}\right\rangle$ mayor es la independencia funcional entre coordenadas. Si se logra reducir $\left\langle\varepsilon_{\text {cont }}\right\rangle$ a medida que se incorporan nuevas componentes, con apropiados $\tau_{j}$, al vector de estado, se estará acomodando óptimamente la reconstrucción del espacio de estados. Una forma de precisar el valor idóneo de $\tau$ apunta al máximo local de $\left\langle\varepsilon_{\text {cont }}\right\rangle$ vs. $\tau$ que asegura la independencia funcional entre las componentes del vector de estado. Si $\left\langle\varepsilon_{\text {cont }}\right\rangle$ permanece a un valor pequeño con un valor de $\tau$ grande, ya no es necesario incluir nuevas componentes al vector de estado, y, por consiguiente, $m=m^{\prime}$.

Los datos disponibles determinan la escala $\varepsilon_{\text {cont }}$ más pequeña para la que es posible establecer una relación funcional. Si la sucesión temporal es demasiado corta para soportar una reconstrucción, el valor de $\varepsilon_{\text {cont }}$ será grande y se distribuirá uniformemente con independencia del valor de $\tau$ escogido. En cualquier caso este método, basado en un estadístico probabilístico, queda completamente caracterizado por la hipótesis nula y el nivel de confianza, pues determinan el número de puntos, a nivel local, necesarios para rechazar la hipótesis nula y admitir un mapeo continuo a una escala $\varepsilon_{\text {cont. }}$ El nivel de confianza que suele emplearse corresponde al típico de un análisis estadístico rutinario, o sea, $\alpha=0,05$ (95\%) o $\alpha=0,01$ (99\%), si bien cada 
aplicación delimita el valor de $\alpha$ que se adapta más fielmente a la reconstrucción del atractor, lo que puede llevar en ocasiones a la teoría de la decisión [Jaynes, 2003], o al análisis de riesgos, a la hora de evaluar los pros y los contras de las diferentes decisiones a las que habría de enfrentarse el experimentador.

En sucesiones temporales que reproducen un sistema caótico, con un número finito de datos de resolución igualmente finita, la pérdida de relación funcional entre las componentes de un vector de estado no solo se debe a la independencia estadística que pueda procurar $\left\langle\varepsilon_{\text {cont }}\right\rangle$, sino también al submuestreo al que se expone el sistema dinámico cuando se emplean valores de $\tau$ grandes. Con valores grandes de $\tau$ es probable que dentro de la ventana temporal que abarca un vector de estado el sistema dinámico experimente tanto un proceso de expansión como un proceso de plegado, característico de los sistemas caóticos. De esta forma, algunas de las componentes del vector de estado sugieren un comportamiento aleatorio, pues por efecto del plegado la localización de los estados se asemeja a una distribución aleatoria, lo que se traduce en una sobreestimación de la dimensión de reconstrucción $m$ [Olbrich and Kantz, 1997]. Con objeto de detectar cuándo las componentes de un vector de estado irrumpen en un régimen de submuestreo, se define un estadístico $\Gamma$, que evalúa si las separaciones entre las componentes de un vector de estado $\mathbf{x}_{t_{0}} \in \mathbb{R}^{m^{\prime}}$ y las componentes de su vecino más próximo en $\mathbb{R}^{m^{\prime}+1}$ son similares a las que muestran puntos distribuidos aleatoriamente en el atractor. Por tanto, se establece una hipótesis nula por la que al menos una de las componentes del vector de estado se distribuye aleatoriamente en el atractor. Así, se determina la distribución de probabilidad de la separación existente entre puntos escogidos aleatoriamente de las $M$ sucesiones temporales. De cada una de las sucesiones temporales se calcula la distribución de probabilidad $\rho_{i}(x)$ de los valores presentes, para $i=1, \ldots, M$, conforme a una clasificación de los mismos por rango o clase (binning), procediéndose acto seguido a calcular la distribución de las separaciones $\sigma_{i}(\xi)$ entre puntos escogidos al azar, esto es,

$$
\sigma_{i}(\xi)=\int \rho_{i}(x) \rho_{i}(\xi-x) \mathrm{d} x .
$$

La probabilidad de que la separación entre los valores de la $j$-ésima componente, escogida al azar de la $i$-ésima sucesión temporal, de un vector de estado $\mathbf{x}_{t_{0}} \mathrm{y}$ de su vecino más cercano, sea menor o igual que $\xi_{j}$ asciende a

$$
\Gamma_{j}=\int_{0}^{\xi_{j}} \sigma_{i_{j}}(\zeta) \mathrm{d} \zeta,
$$

siendo el estadístico $\Gamma=\operatorname{máx}\left(\Gamma_{j}\right)$. Al igual que con el estadístico $\varepsilon_{\text {cont }}$, el valor de $\Gamma$ se promedia entre los diferentes puntos de referencia que conforman el test. El nivel de confianza que suele usarse es $\alpha=0,05$ (95\%), de forma que si $\Gamma \leqslant 0,05$, se rechaza la hipótesis nula, admitiéndose que los retardos $\tau_{j}$ no son demasiado grandes como para que las distancias que separan las componentes del vector de estado puedan atribuirse a una distribución aleatoria. Un indicador de que ha de detenerse el proceso de añadir nuevas componentes al vector de estado se adquiere del fuerte incremento que experimenta la representanción de $\Gamma$ vs. $\tau$.

En suma el estadístico $\left\langle\varepsilon_{\text {cont }}\right\rangle$, una generalización del concepto de correlación lineal, conforme se anexionan nuevas componentes al vector de estado, reduce su 
valor a un mínimo que se mantiene prácticamente a un nivel constante con $\tau$ cuando se alcanza el valor óptimo de $m$. Normalmente se emplea un valor de $\tau_{1}=0$ para la primera componente del vector de estado. Los valores de los diferentes $\tau_{j}$ suelen corresponderse con los máximos globales del estadístico $\left\langle\varepsilon_{\text {cont }}\right\rangle$, si bien en muchos casos podría optarse por máximos locales, en función del trazado sinuoso descrito por $\left\langle\varepsilon_{\text {cont }}\right\rangle$. En sistemas caóticos el estadístico $\left\langle\varepsilon_{\text {cont }}\right\rangle$ incrementa su valor con $\tau$ debido al submuestreo en que incurre el sistema dinámico, por lo que ha de requerirse la asistencia del estadístico $\Gamma$ para recuperar el valor de $\tau$ a partir del cual aquel dispara su valor. El método no implica escalas de tiempo arbitrarias, como sucede con otros métodos heurísticos, sino criterios probabilísticos con los que cotejar la calidad de la reconstrucción del espacio de estados. 


\section{Referencias}

[Abarbanel et al., 1993] Abarbanel, H. D. I., Brown, R., Sidorowich, J. J., and Tsimring, L. S. (1993). The analysis of observed chaotic data in physical systems. Reviews of Modern Physics, 65(4):1331-1392. (páginas 52, 57, 58, 103, 165).

[Albano et al., 1988] Albano, A. M., Muench, J., Schwartz, C., Mees, A. I., and Rapp, P. E. (1988). Singular-value decomposition and the grassberger-procaccia algorithm. Physical Review A, 38(6):3017-3026. (página 74).

[Aleksić, 1991] Aleksić, Z. (1991). Estimating the embedding dimension. Physica D: Nonlinear Phenomena, 52(2-3):362-368. (página 73).

[Broomhead and King, 1986] Broomhead, D. and King, G. P. (1986). Extracting qualitative dynamics from experimental data. Physica D: Nonlinear Phenomena, 20(2-3):217-236. (páginas 53, 60, 74).

[Broomhead et al., 1987] Broomhead, D. S., Jones, R., and King, G. P. (1987). Topological dimension and local coordinates from time series data. Journal of Physics A: Mathematical and General, 20(9):563-569. (página 63).

[Buzug and Pfister, 1992] Buzug, T. and Pfister, G. (1992). Comparison of algorithms calculating optimal embedding parameters for delay time coordinates. Physica D: Nonlinear Phenomena, 58(1-4):127-137. (página 56).

[Buzug et al., 1990] Buzug, T., Reimers, T., and Pfister, G. (1990). Optimal reconstruction of strange attractors from purely geometrical arguments. EPL (Europhysics Letters), 13(7):605-610. (página 56).

[Casdagli et al., 1991] Casdagli, M., Eubank, S., Farmer, J. D., and Gibson, J. (1991). State space reconstruction in the presence of noise. Physica D: Nonlinear Phenomena, 51(1-3):52-98. (páginas 45, 53).

[Ding et al., 1993] Ding, M., Grebogi, C., Ott, E., Sauer, T., and Yorke, J. A. (1993). Plateau onset for correlation dimension: When does it occur? Physical Review Letters, 70(25):3872-3875. (página 54).

[Eckmann and Ruelle, 1985] Eckmann, J.-P. and Ruelle, D. (1985). Ergodic theory of chaos and strange attractors. Reviews of Modern Physics, 57(3):617-656. (páginas $36,47,105,106,165,213)$. 
[Eckmann and Ruelle, 1992] Eckmann, J.-P. and Ruelle, D. (1992). Fundamental limitations for estimating dimensions and lyapunov exponents in dynamical systems. Physica D: Nonlinear Phenomena, 56(2-3):185-187. (páginas 68, 105).

[Fraedrich, 1986] Fraedrich, K. (1986). Estimating the dimensions of weather and climate attractors. Journal of the Atmospheric Sciences, 43(5):419-432. (página $55)$.

[Fraser, 1989a] Fraser, A. M. (1989a). Information and entropy in strange attractors. IEEE Transactions on Information Theory, 35(2):245-262. (páginas 53, 58).

[Fraser, 1989b] Fraser, A. M. (1989b). Reconstructing attractors from scalar time series: A comparison of singular system and redundancy criteria. Physica D: Nonlinear Phenomena, 34(3):391-404. (páginas 53, 63).

[Fraser and Swinney, 1986] Fraser, A. M. and Swinney, H. L. (1986). Independent coordinates for strange attractors from mutual information. Physical Review A, 33(2):1134-1140. (páginas 48, 58).

[Gibson et al., 1992] Gibson, J. F., Farmer, J. D., Casdagli, M., and Eubank, S. (1992). An analytic approach to practical state space reconstruction. Physica D: Nonlinear Phenomena, 57(1-2):1-30. (página 74).

[Gleick, 2012] Gleick, J. (2012). Caos: La creación de una ciencia (Spanish Edition). Grupo Planeta Spain. (página 48).

[Grassberger, 1985] Grassberger, P. (1985). Generalizations of the hausdorff dimension of fractal measures. Physics Letters A, 107(3):101-105. (páginas 65, 123, 132).

[Grassberger, 1988] Grassberger, P. (1988). Finite sample corrections to entropy and dimension estimates. Physics Letters A, 128(6-7):369-373. (páginas 67, 122, 213).

[Grassberger and Procaccia, 1983] Grassberger, P. and Procaccia, I. (1983). Measuring the strangeness of strange attractors. Physica D: Nonlinear Phenomena, 9(1-2):189-208. (páginas 66, 116, 121, 213, 213).

[Grassberger et al., 1991] Grassberger, P., Schreiber, T., and Schaffrath, C. (1991). Nonlinear time sequence analysis. International Journal of Bifurcation and Chaos, 01(03):521-547. (páginas 59, 64, 74, 213).

[Jaynes, 2003] Jaynes, E. T. (2003). Probability Theory: The Logic of Science. Cambridge University Press. (página 77).

[Kantz and Schreiber, 1995] Kantz, H. and Schreiber, T. (1995). Dimension estimates and physiological data. Chaos: An Interdisciplinary Journal of Nonlinear Science, 5(1):143-154. (páginas 65, 164).

[Kantz and Schreiber, 2003] Kantz, H. and Schreiber, T. (2003). Nonlinear Time Series Analysis. Cambridge University Press. (páginas 36, 52, 55, 59, 65). 
[Kaplan and Glass, 1992] Kaplan, D. T. and Glass, L. (1992). Direct test for determinism in a time series. Physical Review Letters, 68(4):427-430. (página 65).

[Kember and Fowler, 1993] Kember, G. and Fowler, A. C. (1993). A correlation function for choosing time delays in phase portrait reconstructions. Physics Letters A, 179(2):72-80. (página 56).

[Kennel and Abarbanel, 2002] Kennel, M. B. and Abarbanel, H. D. I. (2002). False neighbors and false strands: A reliable minimum embedding dimension algorithm. Physical Review E, 66(2). (página 63).

[Kennel et al., 1992] Kennel, M. B., Brown, R., and Abarbanel, H. D. I. (1992). Determining embedding dimension for phase-space reconstruction using a geometrical construction. Physical Review A, 45(6):3403-3411. (páginas 63, 65).

[Kugiumtzis, 1996] Kugiumtzis, D. (1996). State space reconstruction parameters in the analysis of chaotic time series - the role of the time window length. Physica D: Nonlinear Phenomena, 95(1):13-28. (páginas 45, 72, 75).

[Liebert et al., 1991] Liebert, W., Pawelzik, K., and Schuster, H. G. (1991). Optimal embeddings of chaotic attractors from topological considerations. EPL (Europhysics Letters), 14(6):521. (páginas 48, 63).

[Liebert and Schuster, 1989] Liebert, W. and Schuster, H. G. (1989). Proper choice of the time delay for the analysis of chaotic time series. Physics Letters A, 142(23):107-111. (página 58).

[Mandelbrot, 1983] Mandelbrot, B. B. (1983). The Fractal Geometry of Nature. W. H. Freeman. (páginas 33, 71, 112).

[Mañé, 1981] Mañé, R. (1981). On the dimension of the compact invariant sets of certain non-linear maps. In Lecture Notes in Mathematics, pages 230-242. Springer Berlin Heidelberg. (páginas 47, 54).

[Olbrich and Kantz, 1997] Olbrich, E. and Kantz, H. (1997). Inferring chaotic dynamics from time-series: On which length scale determinism becomes visible. Physics Letters A, 232(1-2):63-69. (página 77).

[Packard et al., 1980] Packard, N. H., Crutchfield, J. P., Farmer, J. D., and Shaw, R. S. (1980). Geometry from a time series. Physical Review Letters, 45(9):712-716. (páginas 47, 47, 48, 53).

[Passamante et al., 1989] Passamante, A., Hediger, T., and Gollub, M. (1989). Fractal dimension and local intrinsic dimension. Physical Review A, 39(7):3640-3645. (página 73).

[Pecora et al., 1995] Pecora, L. M., Carroll, T. L., and Heagy, J. F. (1995). Statistics for mathematical properties of maps between time series embeddings. Physical Review E, 52(4):3420-3439. (página 76). 
[Pecora et al., 2007] Pecora, L. M., Moniz, L., Nichols, J., and Carroll, T. L. (2007). A unified approach to attractor reconstruction. Chaos: An Interdisciplinary Journal of Nonlinear Science, 17(1):013110. (página 75).

[Pecora et al., 2009] Pecora, L. M., Moniz, L., Nichols, J., and Carroll, T. L. (2009). A unified approach to attractor reconstruction. In Dana, S. K., Roy, P. K., and Kurths, J., editors, Complex Dynamics in Physiological Systems: From Heart to Brain, pages 3-19, Dordrecht. Springer Netherlands. (páginas 51, 52).

[Plotnick et al., 1996] Plotnick, R. E., Gardner, R. H., Hargrove, W. W., Prestegaard, K., and Perlmutter, M. (1996). Lacunarity analysis: A general technique for the analysis of spatial patterns. Physical Review E, 53(5):5461-5468. (página $71)$.

[Prichard and Theiler, 1995] Prichard, D. and Theiler, J. (1995). Generalized redundancies for time series analysis. Physica D: Nonlinear Phenomena, 84(3-4):476493. (página 59).

[Provenzale et al., 1992] Provenzale, A., Smith, L. A., Vio, R., and Murante, G. (1992). Distinguishing between low-dimensional dynamics and randomness in measured time series. Physica D: Nonlinear Phenomena, 58(1-4):31-49. (páginas 69, 170).

[Rosenstein et al., 1994] Rosenstein, M. T., Collins, J. J., and Luca, C. J. D. (1994). Reconstruction expansion as a geometry-based framework for choosing proper delay times. Physica D: Nonlinear Phenomena, 73(1-2):82-98. (página 56).

[Sauer and Yorke, 1993] Sauer, T. and Yorke, J. A. (1993). How many delay coordinates do you need? International Journal of Bifurcation and Chaos, 03(03):737744. (página 54).

[Sauer et al., 1991] Sauer, T., Yorke, J. A., and Casdagli, M. (1991). Embedology. Journal of Statistical Physics, 65(3-4):579-616. (páginas 39, 48, 51, 54, 75).

[Schreiber, 1995] Schreiber, T. (1995). Efficient neighbor searching in nonlinear time series analysis. International Journal of Bifurcation and Chaos, 5(2):349-358. (página 67).

[Schreiber and Kantz, 1995] Schreiber, T. and Kantz, H. (1995). Noise in chaotic data: Diagnosis and treatment. Chaos: An Interdisciplinary Journal of Nonlinear Science, 5(1):133-142. (página 69).

[Smith, 1988] Smith, L. A. (1988). Intrinsic limits on dimension calculations. Physics Letters A, 133(6):283-288. (página 68).

[Takens, 1981] Takens, F. (1981). Detecting strange attractors in turbulence. In Lecture Notes in Mathematics, pages 366-381. Springer Berlin Heidelberg. (páginas $39,39,47,47,48,51,54,54,75)$.

[Theiler, 1988] Theiler, J. (1988). Lacunarity in a best estimator of fractal dimension. Physics Letters A, 133(4-5):195-200. (página 71). 
[Theiler et al., 1992] Theiler, J., Eubank, S., Longtin, A., Galdrikian, B., and Farmer, J. D. (1992). Testing for nonlinearity in time series: the method of surrogate data. Physica D: Nonlinear Phenomena, 58(1-4):77-94. (páginas 70, 172).

[Tsonis and Elsner, 1988] Tsonis, A. A. and Elsner, J. B. (1988). The weather attractor over very short timescales. Nature, 333(6173):545-547. (páginas 47, 53, $57)$.

[Whitney, 1936] Whitney, H. (1936). Differentiable manifolds. The Annals of Mathematics, 37(3):645. (página 54).

[Yule, 1927] Yule, G. U. (1927). On a method of investigating periodicities in disturbed series, with special reference to wolfer's sunspot numbers. Philosophical Transactions of the Royal Society of London. Series A, Containing Papers of a Mathematical or Physical Character, 226:267-298. (página 47). 



\section{Capítulo 4}

\section{Comportamiento de un sistema dinámico disipativo, recurrente $\mathrm{y}$ ergódico}

La teoría matemática de los sistemas dinámicos constituye un marco de trabajo destinado a concebir y analizar las ecuaciones que modelizan el comportamiento de un proceso físico. Con el tiempo se evidenció que ecuaciones muy simples originaban soluciones muy complejas, atendiendo a la dependencia sensible a las condiciones iniciales, los atractores extraños, con estructura fractal, cuencas de atracción irregulares o secuencias de bifurcación intrincadas. Este bagaje instrumental ha permitido abordar el problema inverso, o sea, a partir del comportamiento observado inferir la causa que lo provoca. Los avances tecnológicos de las últimas décadas han hecho posible que hoy día resulte relativamente fácil disponer de datos experimentales obtenidos de la observación de fenómenos naturales. Los datos adquiridos pueden almacenarse en un ordenador para su posterior análisis. Estos datos capturados, que no son más que mediciones efectuadas a intervalos regulares de tiempo, reciben el nombre de sucesión temporal, y, en cierto sentido, revelan la dinámica generada por el sistema o proceso subyacente. De un examen cuidadoso de los datos se puede extraer información muy valiosa, por ejemplo, si el sistema que generó los datos es determinista y caótico, o, por el contrario, no determinista y aleatorio. En definitiva el análisis de sucesiones temporales desplaza la fundamentación teórica de los sistemas dinámicos a un terreno experimental, menos consolidado que la teoría y con una proyección sin límites.

Cada vez son más las nuevas técnicas específicas de análisis que se plantean, a fin de caracterizar y desentrañar los diferentes comportamientos de los sistemas observados. La disponibilidad de potentes ordenadores ha acercado estas técnicas a muchos científicos e investigadores. No obstante, el análisis de sucesiones temporales es más un arte que una ciencia, y se apela a la prudencia cuando han de interpretarse los resultados que suministran los diferentes tests a los que los datos son sometidos. Es muy fácil caer en la trampa. La literatura está repleta de falsas suposiciones; a veces, uno tiende a ver lo que quiere ver, vencido por las modas imperantes - como lo fue el boom que experimentó el caos, por el que todos los sistemas eran caóticos-, 
y lejos de alcanzarse una lógica rigurosa en la argumentación, se llega a conclusiones erróneas sin significación real.

Tabla 4.1: Comportamientos dinámicos observados.

\begin{tabular}{|c|c|c|}
\hline comportamiento & categoría & ejemplos \\
\hline \multirow{2}{*}{$\begin{array}{l}\text { periódico } \\
\text { cuasiperiódico }\end{array}$} & regular predecible & planetas, relojes, mareas \\
\hline & regular impredecible & lanzamiento de una moneda \\
\hline \multirow[t]{5}{*}{ caótico } & caos transitorio & máquina de pinball \\
\hline & caos intermitente & mapa logístico con $a=3,8284$ \\
\hline & caos de banda estrecha & atractor de Rössler \\
\hline & $\begin{array}{l}\text { caos de baja dimensión } \\
\text { (banda ancha) }\end{array}$ & atractor de Lorenz \\
\hline & $\begin{array}{l}\text { caos de alta dimensión (ban- } \\
\text { da ancha) }\end{array}$ & redes neuronales \\
\hline \multirow[t]{3}{*}{ aleatorio } & ruido coloreado (correlado) & paseo aleatorio \\
\hline & pseudoaleatoriedad & $\begin{array}{l}\text { números «aleatorios» generados } \\
\text { por ordenador }\end{array}$ \\
\hline & ruido aleatorio & radioactividad, estática de radio \\
\hline \multicolumn{2}{|c|}{ combinación de las categorías anteriores } & $\begin{array}{l}\text { la mayoría de los datos del mun- } \\
\text { do real }\end{array}$ \\
\hline
\end{tabular}

Afortunadamente, no todos los sistemas se comportan de manera similar. Ya en 1992 se confeccionó una lista que constaba de siete posibles comportamientos detectados en sistemas no lineales deterministas [Moon, 1992]. A este catálogo se le sumaron otros comportamientos adicionales [Sprott, 2003], como refleja la tabla 4.1. Entre los sistemas más simples figuran los sistemas regulares, como el péndulo, o, incluso, los procesos cuasiperiódicos, como las mareas o las horas a las que se pone el Sol. En el polo opuesto se sitúa la auténtica aleatoriedad, a la que solo es posible acceder a nivel atómico, de acuerdo con las leyes de la mecánica cuántica. Entre ambos extremos se dispone de toda una gama de procesos que, según se acercan a la verdadera aleatoriedad, acentúan su complejidad dinámica en términos de impredecibilidad. En cualquier caso, en el mundo real los procesos suelen exhibir una mezcla de diferentes comportamientos.

Dada la extensión de este capítulo, por su relevancia conceptual, procede perfilar las líneas maestras que apuntalan la teoría de los sistemas dinámicos, si bien algunos 
conceptos clave ya se introdujeron en la sección 2.3. Inicialmente se han planteado las posibles dinámicas hasta la fecha presentes en un sistema físico, como se ilustra en la tabla 4.1, para posteriormente pasar a encarar la transición (teoría de bifurcaciones) entre los comportamientos dinámicos, amén de cómo medir la complejidad de estos comportamientos dinámicos, en términos de entropía, dimensión fractal y exponentes de Lyapunov, antes de concluir con el estudio de dos técnicas de medida de la complejidad, el test $0-1$ y el algoritmo Lempel-Ziv, que permiten discernir las posibles dinámicas que concurren en muchas de las señales del mundo real.

\subsection{Bifurcaciones y rutas al caos}

La tabla 4.1 resume las principales dinámicas descubiertas hasta ahora, si bien este repertorio no ha de ser definitivo. Investigaciones futuras podrían revelar nuevas conductas en los sistemas dinámicos. La naturaleza siempre depara nuevas sorpresas, y es una obligación permanecer atentos para no dejar pasar la oportunidad.

En cualquier sistema en que dos o más frecuencias se acoplan no linealmente puede acontecer toda una variedad de efectos dinámicos, como el enganche en frecuencia (sincronización), la cuasiperiodicidad, la intermitencia, el doblamiento de período o generación de subarmónicos, y el caos [Glazier and Libchaber, 1988, Romera García, 1997]. Los mapas de la circunferencia constituyen un valioso modelo matemático abstracto para explicar no solo el enganche en frecuencia sino también la transición de la cuasiperiodicidad al caos [Arnold, 1965, Jensen et al., 1984], a diferencia de otros modelos también conocidos, que, como en el caso del oscilador de van der Pol [Mettin et al., 1993], reclaman, en el análisis, una engorrosa integración numérica de una ecuación diferencial.

\subsubsection{Bifurcaciones}

Una bifurcación comporta un cambio cualitativo en el comportamiento dinámico de un sistema físico (geometría del atractor) cuando alguno, o varios, de los parámetros de control atraviesa un valor crítico. Los puntos del espacio paramétrico en los que el sistema es estructuralmente inestable se denominan puntos de bifurcación o, simplemente, bifurcaciones. Las bifurcaciones comprenden un signo incontestable de determinismo si el sistema responde coherentemente a variaciones del parámetro de control alrededor de un valor crítico, sin olvidar que muchos sistemas exhiben histéresis (ausencia de reversibilidad) y, por tanto, las bifurcaciones se producen en valores diferentes de los parámetros dependiendo de la dirección en que son modificados los mismos. Desde una perspectiva operativa, resulta trascendental conocer los puntos de bifurcación, con objeto de evaluar sus posibles efectos en la estructura dinámica del sistema físico bajo estudio.

Si bien la teoría de la bifurcación se remonta a trabajos de Poincaré [Poincaré, 1885], puede considerarse una disciplina reciente en la que la nomenclatura aún no ha sido establecida completamente, dado que se cree no haberse descubierto todas las posibles bifurcaciones. En cualquier caso se evidencian bifurcaciones tanto en flujos como en mapas, para diferentes dimensiones de los sistemas físicos; eso sí, normalmente se abordan bifurcaciones de codimensión 1, en las que únicamente se 
cambia un solo parámetro de control, manteniendo el resto constantes, dado que son las que se visualizan con más facilidad en entornos experimentales. Además, es habitual abordar analíticamente las bifurcaciones locales, esto es, aquellas en las que los puntos de equilibrio (puntos fijos y órbitas periódicas en un entorno alrededor de los puntos fijos) se crean, se destruyen o cambian su estabilidad, frente a las bifurcaciones globales, que comprometen a toda la órbita entera. En bifurcaciones continuas, también llamadas supercríticas, un autovalor se convierte en estable (parte real negativa) o inestable (parte real positiva); en bifurcaciones discontinuas, denominadas catastróficas o subcríticas, ${ }^{1}$ los autovalores aparecen o desaparecen.

Para el estudio de las bifurcaciones se suelen acometer inicialmente las bifurcaciones locales en flujos, luego en mapas, para finalmente concluir con las bifurcaciones globales [Crawford, 1991]. Adicionalmente, por motivos de claridad, solo se consideran las formas normales [Wiggins, 1996], es decir, se analizan los modelos polinómicos más simples que exhiben un tipo de bifurcación particular. Estos modelos son prototípicos o representativos de la evolución del comportamiento dinámico en las inmediaciones de los puntos de bifurcación y posibilitan un análisis pormenorizado del cambio sustancial de la dinámica del sistema, dado el carácter universal de las bifurcaciones, a saber, todos los sistemas son equivalentes en la vecindad de un punto de bifurcación. En todo caso esta breve exploración se limita a revisar conceptualmente aquellas bifurcaciones más comunes, a fin de clarificar el análisis posterior de las rutas al caos. En este sentido los diagramas de bifurcación son de gran utilidad, pues muestran en una única representación gráfica las posiciones de los puntos fijos en función del parámetro de control; si los puntos fijos son estables, la línea es continua, si son inestables, la línea es discontinua.

\subsubsection{Bifurcación tangente (saddle-node bifurcation)}

La bifurcación tangente es aquella en la que dos puntos fijos, uno estable y otro inestable, aparecen o desaparecen (ver figuras 4.1a-4.1c). En este último caso, cuando el parámetro es variado, los dos puntos fijos se aproximan hasta colisionar, aniquilándose mutuamente.

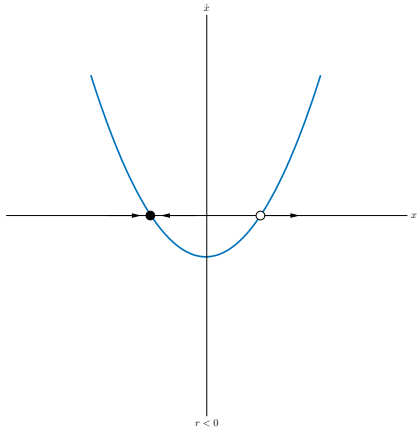

(a) Para $r<0$.

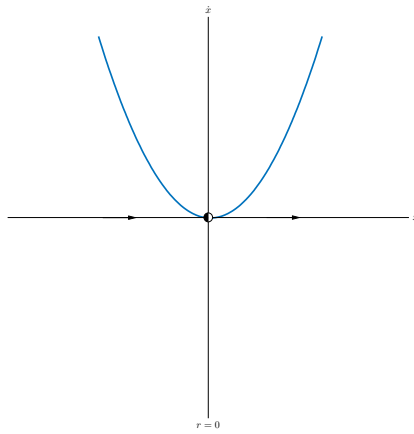

(b) Para $r=0$.

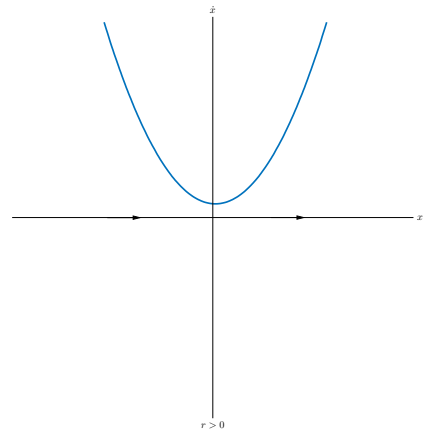

(c) Para $r>0$.

Figura 4.1: Bifurcación tangente de la ecuación $\dot{x}=r+x^{2}$.

\footnotetext{
${ }^{1}$ Una bifurcación discontinua que carece de histéresis recibe el nombre de bifurcación explosiva [Smale, 1967].
} 


\subsubsection{Bifurcación transcrítica (transcritical bifurcation)}

La bifurcación transcrítica es aquella en la que dos puntos fijos intercambian su estabilidad (ver figuras 4.2a-4.2c). A diferencia de la bifurcación tangente, los dos puntos fijos no desaparecen tras la bifurcación, simplemente conmutan su estabilidad.

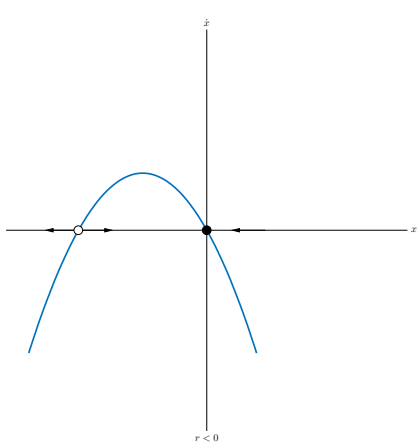

(a) Para $r<0$.

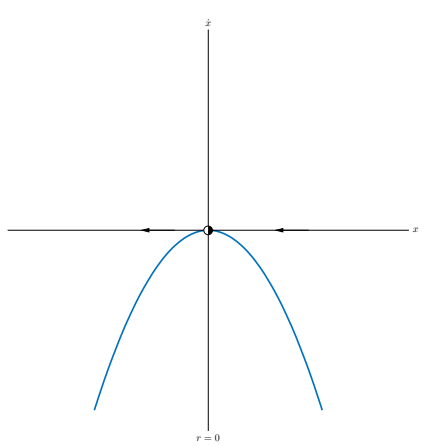

(b) Para $r=0$.

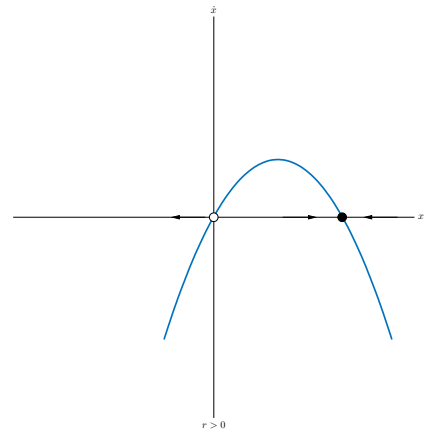

(c) Para $r>0$.

Figura 4.2: Bifurcación transcrítica de la ecuación $\dot{x}=r-x^{2}$.

La bifurcación transcrítica es imperfecta puesto que es estructuralmente inestable, esto es, cualquier ligera perturbación puede hacer desaparecer la bifurcación o convertirla en una bifurcación tangente.

\subsubsection{Bifurcación horca (pitchfork bifurcation)}

La bifurcación horca o tridente es aquella en la que un punto fijo se desdobla en dos. Se origina una bifurcación horca cuando un punto fijo estable se vuelve inestable al mismo tiempo que aparecen dos nuevos puntos fijos estables (ver figuras 4.3a-4.3c). Este tipo de bifurcación se manifiesta típicamente en sistemas físicos que guardan cierta antisimetría espacial, es decir, $f(x)=-f(-x)$.

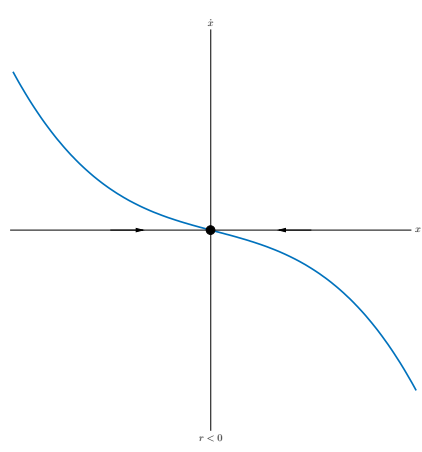

(a) Para $r<0$.

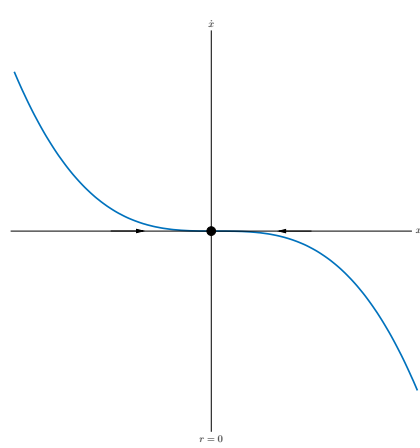

(b) Para $r=0$.

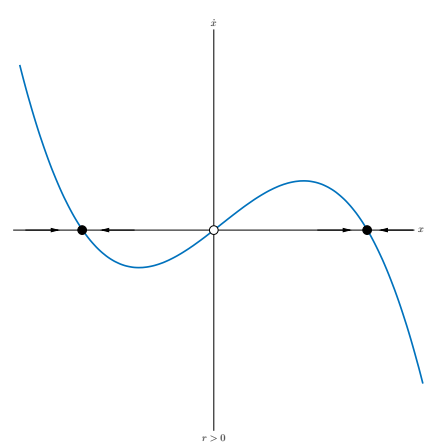

(c) Para $r>0$.

Figura 4.3: Bifurcación horca de la ecuación $\dot{x}=r x-x^{3}$.

Existen dos tipos diferentes de bifurcación horca: la supercrítica y la subcrítica. En la variante supercrítica, para $r>0$ el punto fijo, inicialmente estable, se vuelve inestable y surgen dos nuevos puntos fijos estables (ver figura 4.3c); en la variante 
subcrítica (ver figuras 4.4a-4.4c), con $r<0$, el punto fijo inicialmente estable comparte dinámica con dos puntos fijos inestables, pero para $r>0$ solo está presente un punto fijo inestable, lo que en principio podría conducir al sistema a una dinámica explosiva, de modo que, en tiempo finito, este se alejaría hacia el infinito. Al igual que la bifurcación transcrítica, la bifurcación horca es imperfecta.

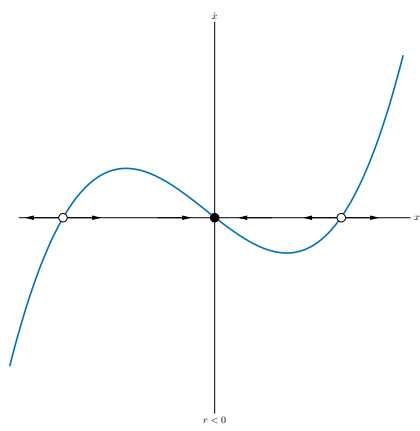

(a) Para $r<0$.

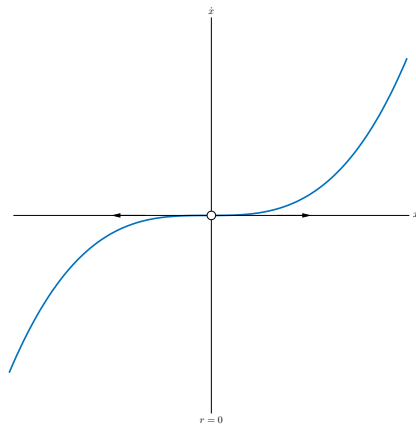

(b) Para $r=0$.

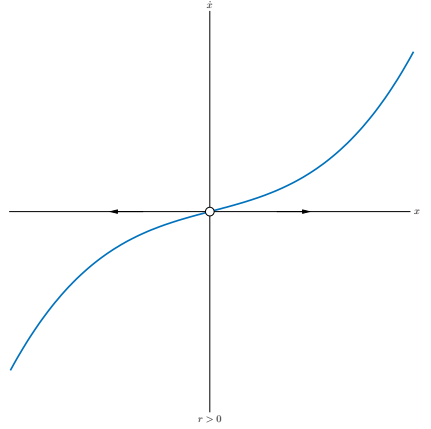

(c) Para $r>0$.

Figura 4.4: Bifurcación horca de la ecuación $\dot{x}=r x+x^{3}$.

\subsubsection{Bifurcación de Hopf (Hopf bifurcation)}

En la bifurcación de Hopf se produce un ciclo límite estable cuando un punto fijo estable se vuelve inestable, generándose una trayectoria que se desarrolla en una espiral ascendente hasta lograr estabilizarse en el ciclo límite, gracias a una no linealidad (ver figuras 4.5a-4.5c). En su variante subcrítica un punto fijo estable y un ciclo límite inestable se aniquilan mutuamente, lo que origina una espiral ascendente sin límite de crecimiento, dinámica explosiva, que progresa, en el peor de los escenarios, hasta el infinito. De algún modo, en una bifurcación subcrítica las trayectorias deben saltar (discontinuidad) a un atractor distante, ya sea un punto fijo, un ciclo límite, un atractor caótico, o, incluso, el infinito.

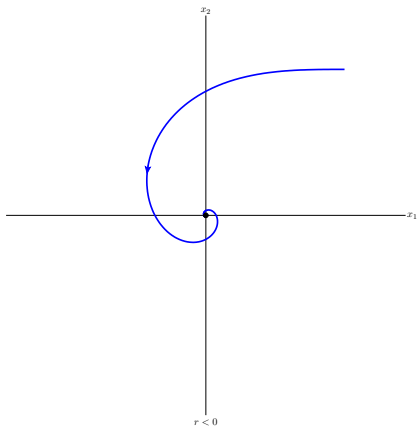

(a) Para $r<0$.

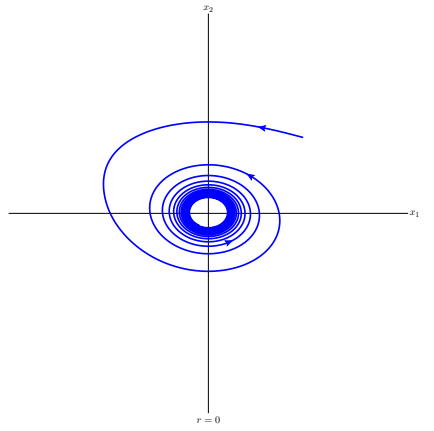

(b) Para $r=0$.

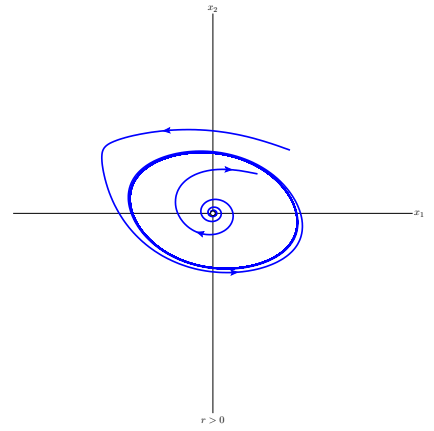

(c) Para $r>0$.

Figura 4.5: Bifurcación bidimensional de Andronov-Hopf, en su variante supercrítica.

En la práctica el ciclo límite, que emerge después de superarse el valor crítico de la 
bifurcación de Hopf, no es circular, sino elíptico, como se reconoce meridianamente en la figura 4.5c. Su tamaño aumenta conforme el valor del parámetro de control se aleja del valor crítico, a la par que se distorsiona su aspecto geométrico, pudiendo variar su frecuencia. Por otro lado, en una bifurcación subcrítica las oscilaciones no crecen sin límite, sino que, normalmente, se ven restringidas por un ciclo límite externo estable de gran amplitud. En este último contexto los sistemas exhiben un comportamiento histerético, o sea, la recuperación de dinámicas previas no se completa en el mismo valor del parámetro de control que disparó la bifurcación. Desde una perspectiva fisiológica, se han encontrado evidencias de bifurcaciones de Hopf subcríticas en la dinámica de células nerviosas [Strogatz, 1994].

En cualquier caso, analíticamente no es fácil distinguir si una bifurcación de Hopf es supercrítica o subcrítica. Experimentalmente, y no sin cierta cautela, si un ciclo límite pequeño que atrae trayectorias se manifiesta inmediatamente después de que el punto fijo se haya vuelto inestable, y su amplitud se reduce a prácticamente cero cuando se revierte el valor del parámetro de control, presumiblemente la bifurcación sea supercrítica. De otra manera, la bifurcación es subcrítica, en cuyo caso el ciclo límite podría estar alejado del punto fijo y el sistema presentaría histéresis.

\subsubsection{Rutas al caos}

La transición entre un estado de equilibrio estable y un comportamiento caótico, entendida en el argot técnico como ruta hacia el caos, se cursa de múltiples formas, a pesar de que, en ocasiones, por su naturaleza abrupta, no se manifieste claramente. En cualquier caso un aspecto interesante de la transición al caos, aparte de su carácter reversible dentro del dominio caótico, radica en la capacidad de identificar, por adelantado, patrones precaóticos, a saber, reconocer la ruta que sigue un sistema dinámico antes de establecerse en un régimen de comportamiento caótico, máxime cuando el mismo sistema dinámico puede adquirir un comportamiento caótico a través de diferentes rutas. Cuando la transición arranca en un estado periódico, las diferentes rutas al caos difieren en la forma en que el régimen periódico pierde estabilidad [Bergé et al., 1987].

Si bien es posible que no se hayan descubierto todas las rutas al caos, como una primera aproximación, tres de ellas, basadas en bifurcaciones locales de codimensión 1, dado que otras configuraciones, muchas veces híbridas, derivan de algún modo de estas tres [Grebogi et al., 1983, Swinney, 1983, Williams, 1997], han recibido especial atención: el doblamiento de período, la intermitencia y la cuasiperiodicidad, aparte de las crisis [Grebogi et al., 1982], que se evidencian poco después de iniciarse el régimen caótico. ${ }^{2}$ Aún no ha podido demostrarse qué tipo de transición es más común [Kadanoff, 1983] en sistemas de dimensión baja, a pesar de que el doblamiento de período sea una de las transiciones más estudiada, y corroborada en infinidad de sistemas físicos de muy diferentes disciplinas científicas. No obstante, en sistemas de

\footnotetext{
${ }^{2}$ Las crisis se atribuyen a colisiones entre un atractor caótico y un punto fijo inestable, o un ciclo límite, coexistente, lo que desencandena un cambio súbito en el atractor caótico. A medida que el sistema se aproxima a la discontinuidad, se manifiesta un caos transitorio, es decir, órbitas aparentemente caóticas decaen exponencialmente hacia órbitas periódicas con una tasa de decaimiento que sigue una ley de potencias en relación con la distancia (en el espacio paramétrico) a la discontinuidad.
} 
dimensión alta prevalece de manera aplastante la cuasiperiodicidad como ruta que lleva al sistema a una dinámica caótica [Doyon et al., 1993].

\subsubsection{Doblamiento de período}

El doblamiento de período conlleva una progresión sistemática, en cascada, hacia el caos. Se ha convertido en el tipo de transición al caos más investigado en un sinnúmero de áreas de conocimiento [Swinney, 1983]. Normalmente, en un rango del parámetro de control, pequeñas variaciones del mismo promueven el doblamiento del período en relación con el período precedente. Además, el doblamiento de período satisface varias leyes de escala universales, que hacen que el comportamiento del sistema, con independencia del proceso físico subyacente, sea idéntico, cualitativa y cuantitativamente [Feigenbaum, 1980].

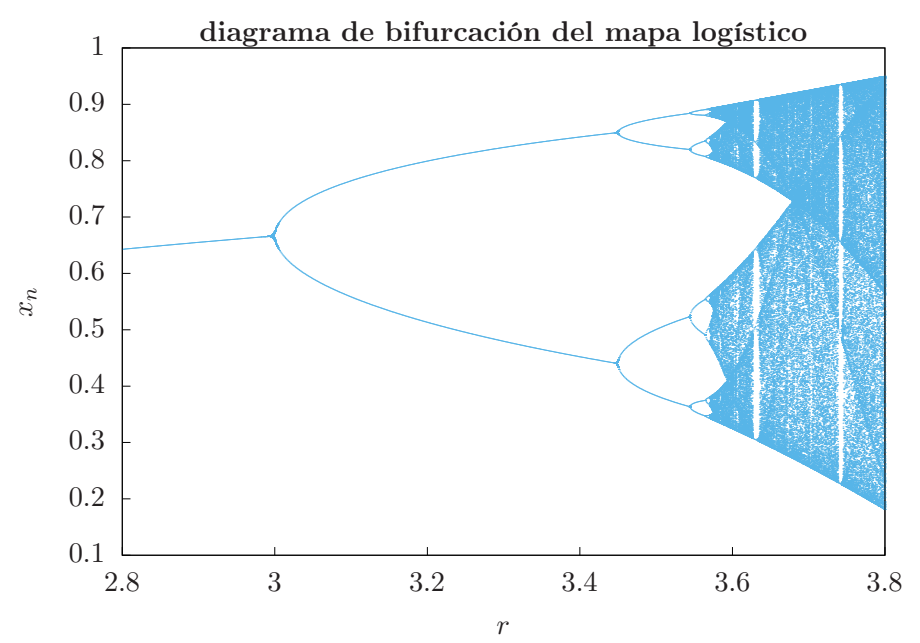

Figura 4.6: Diagrama de bifurcación del mapa logístico $x_{n+1}=r x_{n}\left(1-x_{n}\right)$ para $2,8 \leqslant r \leqslant 3,8$.

Una cascada de doblamiento de período incluye una infinidad de bifurcaciones horca, de forma que en cada una de ellas se dobla el período de la órbita. La cascada de bifurcaciones comenzaría con una bifurcación horca en la que el punto fijo inicialmente estable, después de la primera iteración del sistema, se vuelve inestable tras la segunda iteración, a la par que se crean dos nuevos puntos fijos estables. En una cuarta iteración los dos puntos fijos estables se vuelven inestables al tiempo que se desdoblan en cuatro puntos fijos estables. En general la progresión sistemática satisface que en la $p$-ésima iteración del sistema, donde el período asciende a $p=2^{i}$, para $i=0,1, \ldots$, puntos fijos estables, los $2^{i}$ puntos fijos estables se vuelven inestables y se duplican, obteniéndose $2^{i+1}$ nuevos puntos fijos estables, que conformarían una nueva órbita de período $2 p=2^{i+1}$. Un diagrama de bifurcación ilustrativo de este tipo de cascada de doblamiento de período aparece reflejado en la figura 4.6.

En 1978 el físico y matemático estadounidense Mitchell J. Feigenbaum, durante una estadía en el Laboratorio Nacional de Los Álamos, en el estado de Nuevo México (USA), demostró que en los mapas unimodales, aquellos que solo presentan o bien 
un máximo o bien un mínimo, que dependen de un solo parámetro de control $r$, los valores del parámetro $r_{2^{i}}$, que originan órbitas de períodos $2^{i}$, convergen a un valor $r_{\infty}$, conforme se van doblando escalonadamente los períodos, que descubre una órbita periódica de período infinito, una órbita aperiódica caracterizando el caos [Feigenbaum, 1978] . La constante de Feigenbaum $\delta$, una constante universal, relaciona los valores críticos de $r_{2^{i}}$,

$$
\delta=\lim _{i \rightarrow \infty} \frac{r_{2^{i}}-r_{2^{i-1}}}{r_{2^{i+1}}-r_{2^{i}}}=4,669201609103 \ldots,
$$

de manera que, en el límite asintótico, se corresponde con el inverso de la razón de la progresión geométrica decreciente formada por los términos

$$
r_{2^{i}}-r_{2^{i-1}}, r_{2^{i+1}}-r_{2^{i}}, r_{2^{i+2}}-r_{2^{i+1}}, r_{2^{i+3}}-r_{2^{i+2}}, \ldots
$$

Si se suman los términos de la progresión geométrica, de acuerdo con la ecuación (4.2), se obtiene una predicción de $r_{\infty}$ a partir de dos valores consecutivos del parámetro $r$, esto es,

$$
r_{\infty}-r_{2^{i-1}}=\frac{r_{2^{i}}-r_{2^{i-1}}}{1-1 / \delta}
$$

y, por consiguiente,

$$
r_{\infty}=\frac{\delta r_{2^{i}}-r_{2^{i-1}}}{\delta-1} .
$$

Además, según aumenta el período de la órbita, los rangos de valores de $r$ entre períodos consecutivos se hacen cada vez más pequeños, exigiéndose mayores precisiones para su resolución exacta. A este respecto, la constante de Feigenbaum posibilita computar la duración de un período, de acuerdo con la duración del período precedente. Así,

$$
\text { duración del período } j \times \frac{1}{\delta}=\text { duración del período }(j+1) \text {. }
$$

Para este tipo de transición al caos se han detectado otros números característicos o universales [Feigenbaum, 1978, Collet and Eckmann, 2009], de aplicación en diversos campos científicos, lo que demuestra cómo diferentes ecuaciones no lineales resuelven sus encrucijadas dinámicas de la misma forma.

\subsubsection{Intermitencia}

La intermitencia consiste en una dinámica regular ordenada (ciclo límite), en ausencia de doblamiento de período, interrumpido ocasionalmente, a intervalos irregulares, por ráfagas caóticas o ruido (ver figura 4.7). En sentido matemático, cuando el parámetro de control adopta valores pequeños, solo se manifiesta la dinámica regular. A medida que incrementa el valor del parámetro de control emergen, con carácter excepcional y de forma abrupta, ráfagas caóticas. Incrementos adicionales del parámetro de control vuelven más frecuentes, y de mayor duración, las ráfagas caóticas, hasta que, finalmente, el régimen dinámico es completamente caótico. La 
intermitencia se revela una vez se haya superado ligeramente el valor crítico de una bifurcación tangente, por la que dos puntos fijos, uno estable y otro inestable, se aniquilan mutuamente.

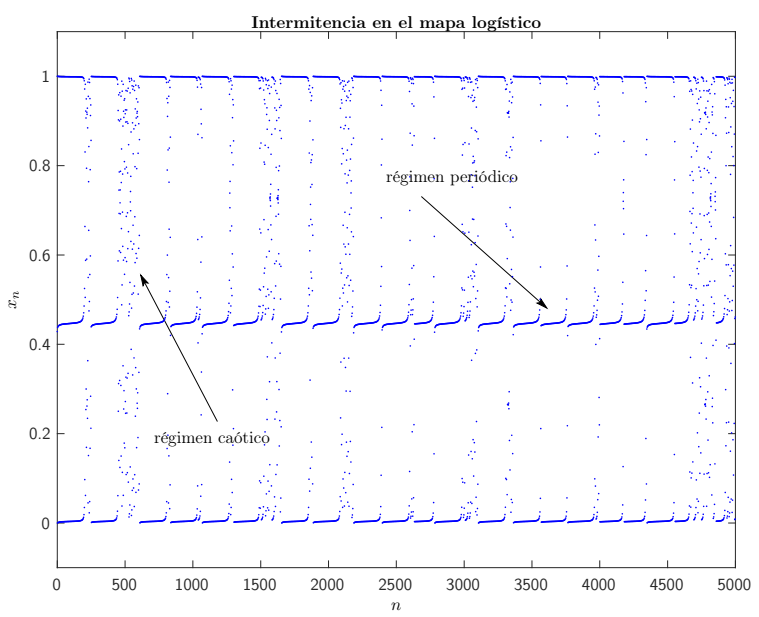

Figura 4.7: Intermitencia del mapa logístico $x_{n+1}=r x_{n}\left(1-x_{n}\right)$, cuando el parámetro de control $r=1+\sqrt{8}-3 \cdot 10^{-5}$.

Durante un tiempo la trayectoria del sistema se despliega en una zona muy delimitada del espacio de estados (ver figura 4.8), en la que las diferentes iteraciones del sistema son prácticamente similares y aparentan describir un comportamiento regular o ciclo límite.

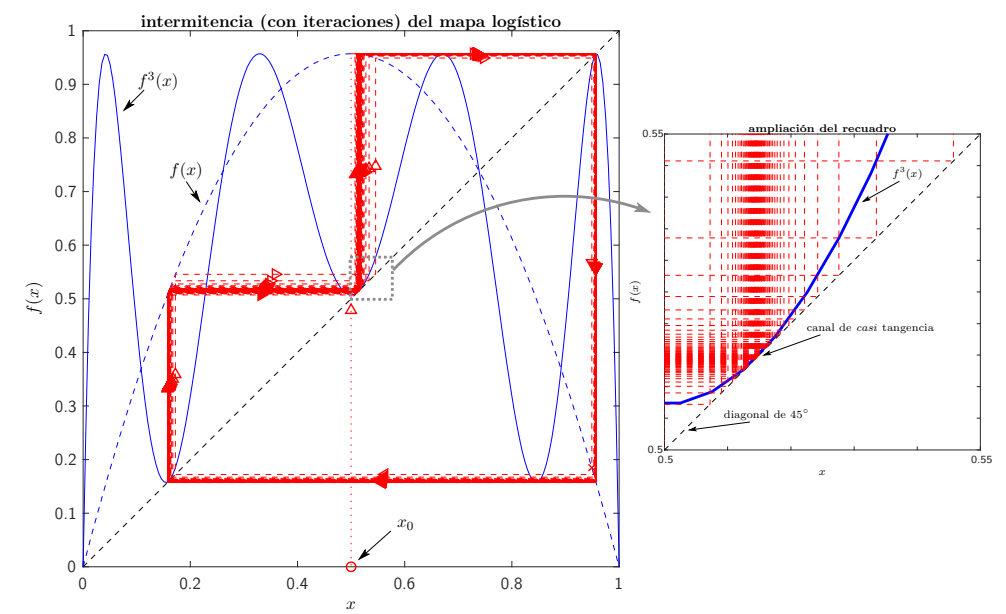

Figura 4.8: Intermitencia, con representación de las iteraciones, del mapa logístico $f(x)=r x(1-x)$, cuando el parámetro de control $r=1+\sqrt{8}-3 \cdot 10^{-5}$, y el valor inicial $x_{0}=0,5$.

Cada vez que la trayectoria logra escapar de esa región estrecha las diferentes iteraciones pueden diferir vastamente entre ellas, con valores muy dispares, pues la trayectoria puede moverse libremente, apenas con restricciones, por el espacio de estados. El sistema se encuentra en una fase caótica, cuya duración es impredecible, 
dado que no se conoce a priori cuándo una de las iteraciones del sistema queda nuevamente atrapada en la región, suscitándose de nuevo un comportamiento regular. Cuanto menor es la diferencia entre el valor del parámetro de control y el valor crítico más estrecha es la región espacial y, por consiguiente, durante más tiempo se mantendría la dinámica regular, puesto que se requieren muchas más iteraciones antes de que el sistema logre abandonar ese comportamiento. En este sentido las etapas regulares son más largas que las caóticas. Conforme aumenta el parámetro de control, la región estrecha se va ensanchando, reduciéndose las iteraciones necesarias para escapar del comportamiento regular, en beneficio de las etapas caóticas, cada vez de mayor duración, hasta que, finalmente, el sistema se comporta de forma caótica en su totalidad. En términos de universalidad se evidencia una ley de escalamiento (ley de potencias) que relaciona la duración media de las etapas regulares con la variación del parámetro de control respecto al valor crítico de la bifurcación tangente [Cvitanović, 1989].

\subsubsection{Transición de la cuasiperiodicidad al caos}

La cuasiperiodicidad es el comportamiento causado por dos o más movimientos periódicos simultáneos, cuyas frecuencias están desfasadas, en modo no proporcional (inconmensurable), entre sí. Dado que las frecuencias son independientes y se carece de un factor común que las relacione las trayectorias nunca se repiten, aunque, a primera vista, parezca que casi lo hacen, de ahí el prefijo cuasi-. Las trayectorias compuestas asociadas a las múltiples periodicidades suelen describir un hipertoroide o $n$-torus (el representativo donut) en el espacio de estados, cuya estructura geométrica es el resultado de $n$ ciclos límite interactuando [Crutchfield et al., 1986]. En el límite asintótico $t \rightarrow \infty$, las trayectorias compuestas cubrirían toda la superficie del toroide, si bien dos trayectorias cualesquiera siempre mantendrían la misma separación entre ellas con el tiempo [Tsonis, 1989].

Desde una perspectiva cronológica, antes de la consolidación de los atractores extraños, la teoría de la turbulencia se asentó inicialmente sobre las propuestas del físico y matemático ruso Lev Landáu (1908-1968), por las que un fluido consistía en infinidad de osciladores de frecuencias inconmensurables que eran excitados conforme se suministraba más energía al sistema. De esta forma, la dinámica del sistema evolucionaba desde un equilibrio estable, pasando por un ciclo límite, un toroide 2-dimensional, un toroide 3-dimensional, etc., hasta que finalmente el espectro de frecuencias era en esencia continuo [Landau and Lifshitz, 1987]. Paralelamente, y de forma independiente, el matemático alemán Eberhard Hopf (1902-1983) había desarrollado una teoría similar [Hopf, 1948]. Sin embargo, el matemático brasileño Maurício Peixoto demostró que un toroide es estable cuando su dimensión es menor que tres. Por otro lado, el matemático francés David Ruelle y el matemático holandés Floris Takens plantearon una ruta alternativa al caos donde un toroide 2-dimensional se convierte en un atractor extraño [Ruelle and Takens, 1971], que no es más que el toroide 2-dimensional al que se le prolongan pliegues, dobleces o arrugas. La ruta al caos sugerida por Lev Landáu no ha sido probada experimentalmente, a pesar de los ímprobos esfuerzos promovidos por los físicos americanos Jerry Gollub y Harry Swinney, de ahí que hoy día sea considerada errónea, a diferencia de la ruta al caos 
de David Ruelle y Floris Takens, que sí ha sido observada experimentalmente en los trabajos emprendidos por los físicos Anke Brandstater y Harry Swinney [Sprott, 2003].

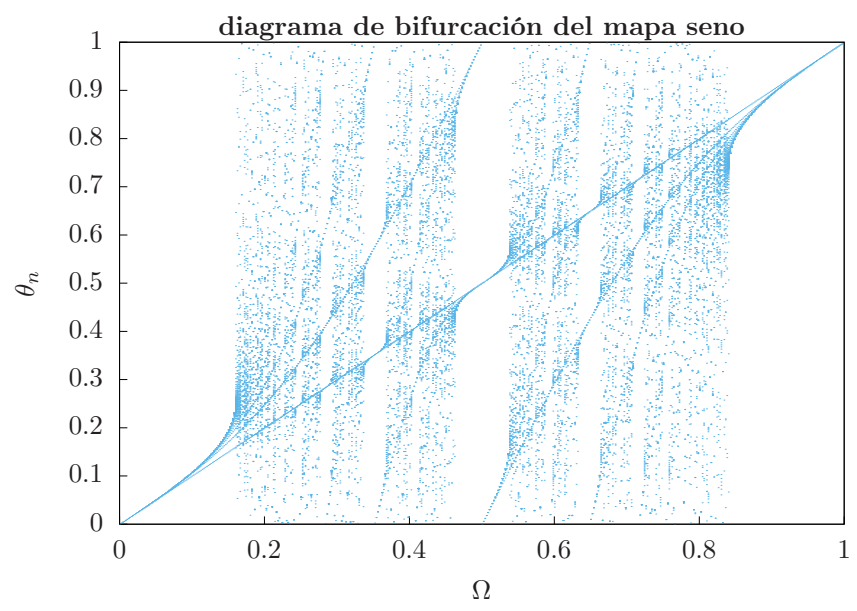

(a) Para $k=1$.

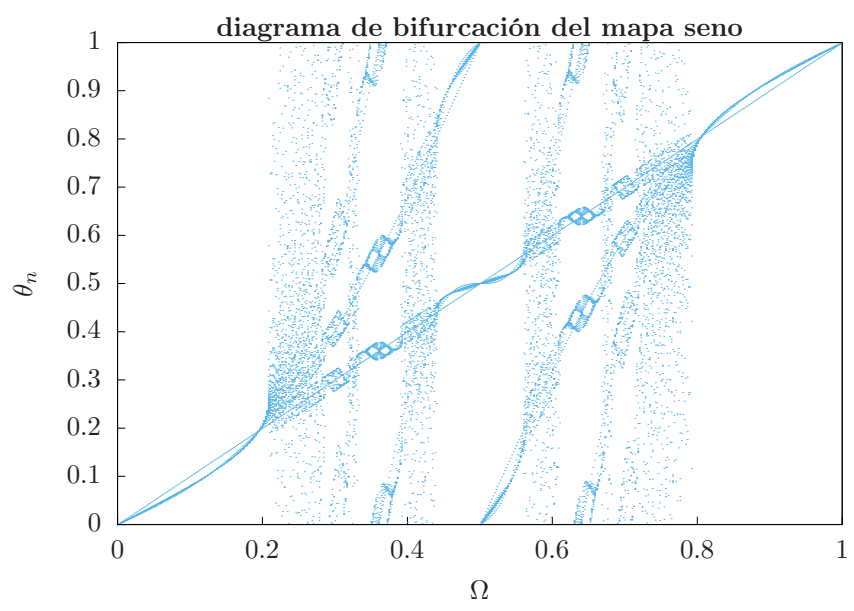

(b) Para $k=1,3$.

Figura 4.9: Ejemplo de transición desde la cuasiperiodicidad al caos en el caso del mapa seno $\theta_{n+1}=\theta_{n}+\Omega-(k / 2 \pi) \sin \left(2 \pi \theta_{n}\right)$ (mód 1$)$, para $k \geqslant 0$. En (a) se obervan claramente las órbitas periódicas (hilos de Arnold); en (b) los hilos de Arnold se desdoblan, multiplicando su periodicidad o volviéndose caóticos.

El desarrollo de la transición al caos, vía la cuasiperiodicidad, se caracteriza por tres hitos cruciales, según aumenta algún parámetro de control del sistema [Grebogi et al., 1983, Robinson, 1982, Schuster and Just, 2006]. Para un valor pequeño del parámetro de control, el sistema se encuentra en un estado estable, un punto fijo en el espacio de estados (frecuencia nula). A medida que se incrementa el valor del parámetro de control, en un momento dado, el primer hito, el sistema se comporta de forma periódica, un ciclo límite, a una frecuencia determinada. Conforme aumenta 
el valor del parámetro de control, el sistema atraviesa el segundo hito, adoptando un comportamiento cuasiperiódico, con dos frecuencias casi idénticas, pero inconmensurables. Si sigue incrementándose el valor del parámetro de control, llega un momento, el tercer hito, en el que pueden desencadenarse dos escenarios. Un primer escenario comprende una desintegración o colapso del toroide bidimensional (2-torus), esto es, el sistema muta su comportamiento, directamente de un régimen cuasiperiódico a un régimen caótico. En otro escenario alternativo, más complejo, antes de que el sistema se sumerja en el régimen caótico, se adhiere al régimen cuasiperiódico una tercera frecuencia. Una vez instalada esta frecuencia adicional, no se sabe con certeza cuándo se inicia el caos, si inmediatamente o si se atraviesan estadios adicionales previos, con más frecuencias e hitos, que, debido al ruido, pudieran quedar enmascarados [Kantz and Schreiber, 2004]. En resumidas cuentas, idealmente la transición al caos experimenta una progresión ordenada desde un estado constante, pasando por una fase periódica y otra cuasiperiódica, hasta alcanzar finalmente el régimen caótico, como ilustran las figuras 4.9a y 4.9b. Cada uno de los hitos atravesados constituye una bifurcación de Hopf. Además, la ruta hacia el caos, por medio de la cuasiperiodicidad, también adquiere ciertas propiedades universales, como sucede en el doblamiento de período con la constante de Feigenbaum. Históricamente, este tipo de transición se observó en dinámica de fluidos, si bien su detección ya se ha extendido a otros sistemas físicos, incluidos los sistemas fisiológicos, como las células cardíacas de los pollos [Schuster and Just, 2006].

\subsection{Grado de complejidad: caracterización de un sistema dinámico}

Las diferentes ramas de la ciencia se han visto salpicadas por la influencia del caos determinista. Toda la fundamentación conceptual e instrumental que conlleva ha impregnado la manera de analizar diferentes sistemas que hasta entonces eran considerados aleatorios. Como objetos matemáticos los sistemas caóticos muestran estructuras muy variopintas y sorprendentes [Alligood et al., 2012]. En el ámbito de las ciencias aplicadas el caos determinista ha estimulado nuevas interpretaciones al comportamiento irregular de ciertos sistemas con anomalías en sus dinámicas, que no obedecen a pautas estrictamente estocásticas.

El vínculo más directo entre la teoría del caos y los fenómenos del mundo real se atribuye sin fisuras al análisis de sucesiones temporales, adquiridas de sistemas reales, en términos de dinámica no lineal. Por un lado, la ciencia experimental y las técnicas de análisis han experimentado un fuerte impulso, motivado, quizás, por la fortuna de haber reunido, no sin esfuerzo y perseverancia, muchas peculiaridades de la dinámica no lineal a nivel de laboratorio, en un entorno muy controlado en el que poder efectuar ensayos que demuestren la presencia de características achacables a ciertos comportamientos observados. Por otro lado, las diferentes investigaciones de las últimas décadas se han empeñado en trasladar las ideas de la teoría del caos, fuertemente consolidada, a casos donde la dinámica no es claramente determinista, si bien los datos reflejan aparentemente cierta regularidad o estructura que, no en pocas circunstancias, puede incluso ser evaluada por métodos tradicionales (lineales). 
Las áreas receptoras de cierto influjo por parte de la teoría del caos han concebido modelos simples, bien a escala microscópica o bien a escala macroscópica o fenomenológica, capaces de desarrollar dinámicas extremadamente complejas. El principal inconveniente radica en confirmar si el modelo ideado corresponde a una señal realmente capturada de la naturaleza. En este sentido puede confirmarse una carencia notable de «buenos» modelos, de ahí que la única alternativa viable, muy común actualmente fuera del entorno del laboratorio, sea estudiar los sistemas a partir de las observaciones recogidas, por medios instrumentales, en una sucesión temporal escalar. La teoría de los sistemas dinámicos no lineales proporciona herramientas muy valiosas para cuantificar y, por ende, caracterizar las irregularidades o anomalías presentes en una sucesión temporal. La variedad de métodos es amplia; estos se extienden desde el cómputo de magnitudes invariantes, como los exponentes de Lyapunov, la entropía, o las dimensiones, con las que poder describir la configuración funcional y estructural del sistema, en caso de disponer de datos de alta calidad, hasta técnicas estadísticas sofisticadas que favorecen la clasificación y el diagnóstico incluso en situaciones en las que el determinismo apenas es perceptible.

La experimentación con señales de la naturaleza, al fin y al cabo, procesos dinámicos no lineales, exige una metodología coherente orientada a la aplicación de técnicas de preprocesado de la señal que eliminen, en la medida de lo posible, las deformaciones a que se ven abocadas las señales adquiridas; diferentes artefactos, acumulados en su recorrido a través del sistema de adquisición de datos, desvirtúan la dinámica real subyacente y son especialmente perjudiciales en áreas como la biología, la fisiología, y la economía, entre otras [Kantz and Schreiber, 2004]. Con las señales más «limpias» si cabe, es posible emprender un análisis exhaustivo de la dinámica subyacente, sin prescindir de una obligada prudencia como consecuencia de la limitada disponibilidad de datos, además de las restricciones computacionales de los algoritmos prescritos [Akay et al., 2000]. En cualquier caso es la naturaleza de los sistemas la que invita, en cierta forma, a un tipo de análisis particularmente beneficioso. Por ejemplo, en metereología se fomentan métodos predictivos a corto y medio plazo [Tsonis, 2012], o en investigación clínica, indicadores pertinentes para el diagnóstico de enfermedades graves, etc.

Diversas medidas de la complejidad permiten comparar diferentes sucesiones temporales y distinguir si su comportamiento es regular, caótico o aleatorio, lo que, en el caso de las señales biológicas, podría ayudar a diagnosticar enfermedades o disfunciones orgánicas. Entre las medidas de la complejidad sobresalen parámetros como la entropía, la dimensión fractal y los exponentes de Lyapunov [Unakafova and Keller, 2013]. Estos parámetros, relacionados entre sí, se evalúan sobre una trayectoria típica (observable medido) de un sistema dinámico presuntamente ergódico.

Las señales biológicas, capturadas normalmente mediante dispositivos hardware en entornos reales, contienen bastante ruido, imposibilitándose la obtención de resultados con sentido cuando los parámetros que miden la complejidad se determinan directamente sobre la base de sucesiones temporales sin preprocesar. Por tanto, un paso previo al análisis de sucesiones temporales requiere un preprocesamiento exhaustivo de los datos, a fin de eliminar el «ruido» presente en los mismos y que, no en pocas situaciones, arrojaría información errónea del sistema dinámico bajo estudio. De acuerdo con esto, cabe aclarar que las señales biológicas que se utilizan 
en esta investigación, una vez adquiridas de los diferentes sujetos, fueron previamente sometidas a un proceso de eliminación del ruido por parte de los responsables del experimento, si bien ciertos artefactos, en virtud de la naturaleza particular de este tipo de señales, fueron reducidos en la medida de lo posible para su correcta aplicabilidad en este trabajo.

\subsubsection{Exponentes de Lyapunov}

La característica más sorprendente del caos es la impredecibilidad del estado futuro de un sistema a pesar de su evolución determinista. El aforismo «causas similares desencadenan efectos similares» no se cumple en sistemas caóticos, excepto en intervalos de tiempo muy cortos. La impredecibilidad que exhiben los sistemas caóticos deriva de la inestabilidad inherente a las soluciones de las ecuaciones que gobiernan su evolución temporal, y que se refleja en lo que se denomina comúnmente sensibilidad a las condiciones iniciales. Por un lado, la impredecibilidad está estrechamente relacionada con la pérdida de información que los sistemas caóticos experimentan, y que queda perfectamente identificada por la entropía de Kolmogórov-Sinái; por otro lado, la impredecibilidad, desde una perspectiva geométrica, se supedita a que trayectorias inicialmente muy próximas se separan muy rápidamente en el tiempo, más concretamente, a un ritmo exponencial.

Los exponentes de Lyapunov cuantifican el grado de separación, en promedio, de trayectorias que emergen de estados iniciales muy próximos en el espacio de estados en que habita un sistema dinámico. Una divergencia exponencial de trayectorias inicialmente cercanas entre sí, junto con el plegado que experimentan, asegurándose así que las soluciones sean finitas, constituye el mecanismo genérico que sustancia el caos determinista. De hecho, la existencia de un exponente de Lyapunov positivo, ${ }^{3}$ para casi todas las condiciones iniciales de un sistema dinámico acotado, se ha convertido en el sello de identidad del caos determinista.

El origen de este indicador del caos se remonta a los trabajos realizados por la matemática rusa Sofya Kovalevskaya (1850-1891) en el siglo XIX. En 1889 Sofya Kovalevskaya definió la inestabilidad dinámica como la tasa promedio de crecimiento de pequeñas desviaciones [Percival, 1989]. El matemático ruso Aleksandr M. Liapunov (1857-1918) extendió la definición de inestabilidad dinámica [Liapounoff, 1948] y, posteriormente, Valery Oseledets estableció los fundamentos teóricos [Oseledets, 1968].

Sean $\vec{x}_{0}(t)$ una trayectoria de referencia que pasa a través de $\vec{x}_{0}(0)$ en el instante de tiempo $t=0$ y $\vec{x}_{1}(t)$ una trayectoria que pasa a través de $\vec{x}_{1}(0)$ en el instante de tiempo $t=0$. El máximo exponente de Lyapunov $\lambda\left(\vec{x}_{0}\right)$ se define respecto a la trayectoria de referencia $\vec{x}_{0}(t)$ como

$$
\lambda\left(\vec{x}_{0}\right)=\lim _{t \rightarrow \infty} \lim _{\|\Delta \vec{x}(0) \rightarrow 0\|} \frac{1}{t} \log \frac{\|\Delta \vec{x}(t)\|}{\|\Delta \vec{x}(0)\|},
$$

donde $\|\Delta \vec{x}(0)\|$ identifica la distancia euclidiana entre la trayectoria $\vec{x}_{1}(t)$ y la trayectoria de referencia $\vec{x}_{0}(t)$ en el instante $t=0$, y $\|\Delta \vec{x}(t)\|$ la distancia euclidiana

\footnotetext{
${ }^{3}$ Según la literatura consultada recibe otras denominaciones, como exponente de Lyapunov característico o simplemente exponente característico.
} 
entre la trayectoria $\vec{x}_{1}(t)$ y la trayectoria de referencia $\vec{x}_{0}(t)$ un tiempo $t$ más tarde.

Conforme a la ecuación (4.6), la trayectoria $\vec{x}_{1}(t)$ suele atender a una trayectoria infinitesimalmente próxima a $\vec{x}_{0}(t)$ en $t=0$. Si se reordenan los términos de la ecuación, la correspondencia entre la sensibilidad a las condiciones iniciales y un exponente de Lyapunov positivo es directa, esto es,

$$
\|\Delta \vec{x}(t)\| \sim\|\Delta \vec{x}(0)\| \exp (\lambda t) .
$$

Un sistema dinámico en $\mathbb{R}^{m}$ cuenta con $m$ exponentes de Lyapunov según la relación de orden

$$
\lambda_{1} \geqslant \lambda_{2} \geqslant \cdots \geqslant \lambda_{m}
$$

Con objeto de determinar el espectro de exponentes de Lyapunov se considera una esfera infinitesimal de $m$ dimensiones como subconjunto de condiciones iniciales asociadas a una trayectoria de referencia. A medida que la esfera evoluciona con el tiempo, esta se deforma hasta convertirse en un elipsoide. Si $p_{i}(t)$ denota la longitud del $i$-ésimo eje principal en un tiempo $t$,

$$
\lambda_{i}=\lim _{t \rightarrow \infty} \frac{1}{t} \log \left(\frac{p_{i}(t)}{p_{i}(0)}\right), \quad i=1,2, \ldots, m,
$$

define el espectro de exponentes de Lyapunov, ordenados de mayor a menor magnitud. A tenor de la ecuación (4.9), un elemento de volumen en el espacio de estados $\mathbb{R}^{m}$ evoluciona en el tiempo de acuerdo con

$$
V(t) \sim V(0) \exp \left(\sum_{i=1}^{m} \lambda_{i} t\right),
$$

donde $V(0)$ representa el volumen en $t=0$ y $V(t)$ el volumen un tiempo $t$ más tarde. La suma de los exponentes de Lyapunov coincide con la divergencia del campo vectorial $\overrightarrow{\mathbf{F}}$, a saber,

$$
\sum_{i=1}^{m} \lambda_{i}=\operatorname{div} \overrightarrow{\mathbf{F}}=\nabla \cdot \overrightarrow{\mathbf{F}} .
$$

Dado que los sistemas dinámicos disipativos, habituales en el mundo real, están acotados en el espacio de estados, la divergencia de las trayectorias, atribuida al caos, se mantiene de forma sistemática durante un tiempo limitado, el tiempo de predicción. Transcurrido ese tiempo, la evolución de las trayectorias abandona el régimen sistemático y se adentra en el terreno de lo incierto donde se hace muy difícil determinar con exactitud en qué región del espacio de estados se encuentra la trayectoria $\vec{x}_{1}(t)$, respecto a la trayectoria de referencia $\vec{x}_{0}(t)$, incluso conociéndose exactamente las ecuaciones que rigen el comportamiento temporal del sistema. Obviamente, una mayor precisión en las medidas de los estados del sistema extiende el horizonte temporal de predicción.

Si se evalúan los exponentes de Lyapunov en las diferentes regiones del atractor en que se desarrolla toda la dinámica de un sistema caótico, aquellos no coinciden, de ahí que reciban la denominación de exponentes locales de Lyapunov [Fujisaka, 
1983, Grassberger and Procaccia, 1984, Nese, 1989, Abarbanel et al., 1992], frente a los exponentes estándar de Lyapunov, que promedian los exponentes locales sobre el conjunto del atractor. Los exponentes de Lyapunov presentan un marcado carácter «local», adoptando diferentes valores dependiendo de la región del espacio de estados en que se estimen. La variabilidad de sus valores adquiere un significado estadístico. La distribución de las frecuencias ligada a esta variabilidad en función del espacio de estados se caracteriza por el factor de no uniformidad (NUF o non-uniformity factor), que identifica la desviación típica de la distribución de frecuencias. El factor NUF constituye un indicador global del nivel de dispersión de los exponentes locales de Lyapunov [Nicolis et al., 1983]. En cualquier caso es conveniente disponer de un único valor promedio que represente a todos los exponentes locales de Lyapunov, el primer y mayor exponente de Lyapunov $\lambda_{1}$. Por tanto, el exponente de Lyapunov $\lambda_{1}$ se considera una característica global e invariante, pues no depende del instante de tiempo en que comienza a monitorizarse su valor. Además, dado que un atractor no crece por igual en todas las dimensiones a medida que evoluciona en el tiempo, el exponente $\lambda_{1}$ identifica la dimensión en la que crece más rápidamente, y, por consiguiente, cuantifica su capacidad predictiva promedio. En este sentido trayectorias muy próximas simbolizan cambios o perturbaciones en las condiciones iniciales y $\lambda_{1}$ una medida global de la sensibilidad del sistema a estos ligeros cambios o perturbaciones. Un sistema dinámico regular no es especialmente sensible a ligeros cambios en las condiciones iniciales, pues trayectorias muy próximas tienden a converger; sin embargo, un sistema dinámico operando en régimen caótico se muestra muy sensible a ligeros cambios en las condiciones iniciales, dado que trayectorias cercanas se separan muy rápidamente.

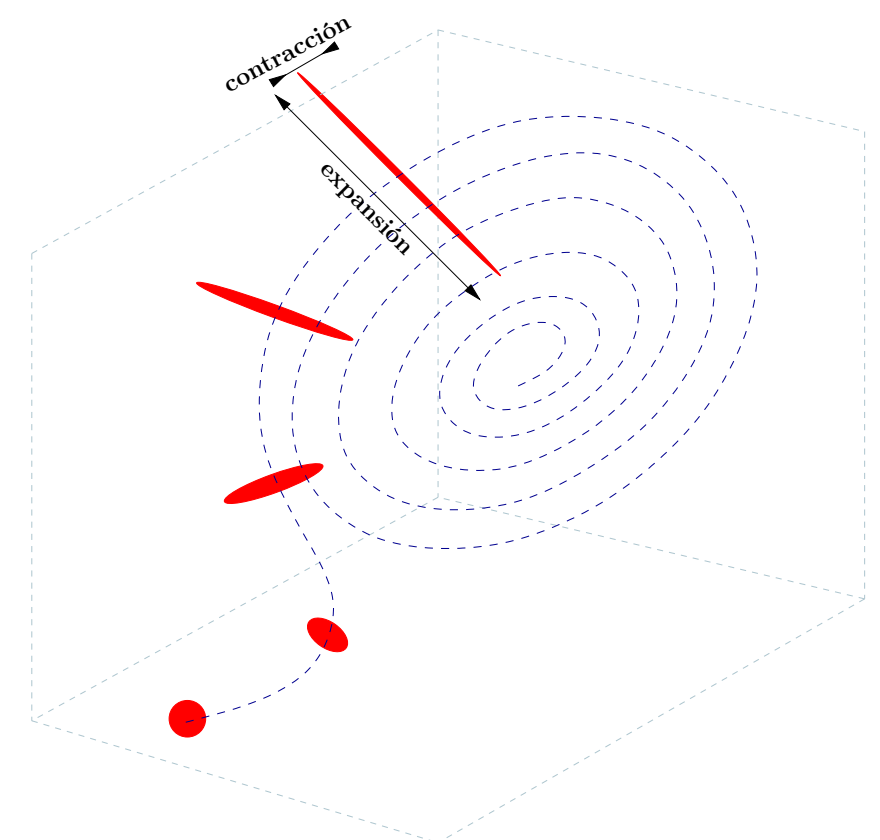

Figura 4.10: Fenómenos de expansión y contracción de una unidad de volumen infinitesimal $\bullet$ describiendo una trayectoria en un espacio de estados. 
El signo y el valor del exponente de Lyapunov determinan la fiabilidad de las predicciones. Un exponente de Lyapunov positivo y de gran magnitud revela una alta sensibilidad a las condiciones iniciales y, en consecuencia, una pobre capacidad predictiva. Valores negativos del exponente de Lyapunov sugieren una convergencia relativamente rápida de las trayectorias próximas y, por tanto, una fiabilidad elevada en términos de predicción.

La deformación que experimenta una unidad de volumen infinitesimal con el tiempo viene caracterizada por unos ejes principales, también llamados vectores de perturbación, como así ilustra la figura 4.10. Estos ejes principales, si bien ortogonales, no tienen por qué coincidir con el sistema de coordenadas de referencia del espacio de estados. Las longitudes y las orientaciones de los ejes principales se modifican de acuerdo con la naturaleza no lineal del sistema dinámico. El eje principal que soporta mayor cambio se denomina primer eje principal y especifica la dirección en la que el flujo dinámico es más inestable. El número de exponentes de Lyapunov coincide con el número de ejes principales o, de otro modo, con el número de direcciones que experimentan alguna modificación conforme evoluciona temporalmente el sistema dinámico. El caos puede acontecer en todo sistema dinámico de cualquier número de dimensiones. Así, puede haber cualquier número de exponentes de Lyapunov positivos, tantos como dimensiones del espacio de estados. Además de exponentes de Lyapunov positivos y negativos también es posible un exponente de Lyapunov cero. Un exponente de Lyapunov cero comprende un eje principal cuya longitud permanece constante, o incrementa con el tiempo a un ritmo menor que el exponencial; es la dirección marginalmente estable. Se ha demostrado matemáticamente que, salvo para el caso de puntos fijos, todo sistema dinámico presenta, al menos, un exponente de Lyapunov cero [Parker et al., 1989]. Por lo tanto, las trayectorias de cualquier sistema dinámico se comportan de tres formas posibles:

1. Asintóticamente inestables. Las trayectorias vecinas divergen exponencialmente con el tiempo. El sistema dinámico se rige por un exponente de Lyapunov positivo.

2. Marginalmente estables. Las trayectorias vecinas evolucionan casi paralelamente. El sistema dinámico se rige por un exponente de Lyapunov cero.

3. Asintóticamente estables. Las trayectorias vecinas convergen exponencialmente con el tiempo. El sistema dinámico se rige por un exponente de Lyapunov negativo.

Un mecanismo para describir cualitativamente un sistema dinámico simplemente lista los signos de los exponentes de Lyapunov. Por ejemplo, un sistema dinámico $(+, 0,-)$ conforma un espacio de estados tridimensional en el que trayectorias vecinas, en media, divergen exponencialmente a lo largo del primer eje principal, son marginalmente estables a lo largo del segundo eje principal, y convergen a lo largo del tercer eje principal. En el caso de sistemas caóticos disipativos cabe destacar que, al menos, un eje principal debe presentar un exponente de Lyapunov con valor cero, y la suma de los exponentes de Lyapunov ha de ser negativa, a fin de asegurar su comportamiento disipativo. Por consiguiente, en un sistema dinámico disipativo la 
suma de los exponentes de Lyapunov es negativa. Además, para trayectorias acotadas que no tienden a un punto fijo, al menos un exponente de Lyapunov ha de ser cero.

\subsubsection{Cálculo de los exponentes de Lyapunov a partir de una sucesión temporal}

Los diferentes métodos que posibilitan el cálculo del espectro de exponentes de Lyapunov se basan en la reconstrucción del espacio de estados a partir del único observable consignado en una típica sucesión temporal $\left\{x\left(t_{n}\right)\right\}$, muestreada en los instantes de tiempo $t_{n}=n \Delta t$, siendo $\Delta t$ el período de muestreo. El método de los retardos, o de las coordenadas retardadas (delay coordinates), proporciona una matriz de trayectorias $X$, de orden $N_{r} \times m$, en un espacio de estados reconstruidos,

$$
X=\left(\mathbf{x}_{1}, \mathbf{x}_{2}, \ldots, \mathbf{x}_{N_{r}}\right)^{T},
$$

donde cada fila de la matriz identifica, para cada instante de tiempo discreto $n$, un vector de estado reconstruido $\mathbf{x}_{n}=\left[x\left(t_{n}\right), x\left(t_{n+\tau}\right), \ldots, x\left(t_{n+(m-1) \tau}\right)\right]{ }^{4} \mathrm{~A}$ partir de una sucesión temporal $\left\{x\left(t_{n}\right)\right\}$, de longitud $N$, con una dimensión de reconstrucción $m$ y un retardo $\tau$, pueden reconstruirse $N_{r}=N-(m-1) \tau$ vectores de estado. La fiabilidad de la reconstrucción del espacio de estados se supedita a que la ventana temporal $(m-1) \tau$ de cada vector de estado reconstruido retenga las escalas de tiempo en que se desenvuelve la dinámica del sistema, como el primer cero o el primer mínimo de la función de autocorrelación, o el primer mínimo de la información mutua [Abarbanel et al., 1993, Kantz and Schreiber, 2004]. Dado que los exponentes de Lyapunov son invariantes respecto a transformaciones difeomórficas del sistema de coordenadas [Munkres, 2000], los exponentes de Lyapunov estimados en un espacio de estados reconstruidos son equivalentes a los que se obtendrían si se dispusiese del sistema de coordenadas natural.

Aun pudiéndose reconstruir el espacio de estados, no se dispone de un criterio veraz que corrobore la equivalencia perfecta con el espacio de estados original. En cualquier caso cada punto de la trayectoria es considerado como el centro de un volumen infinitesimal, caracterizado por sus ejes principales, a priori ortogonales. La evolución del sistema dinámico deforma el volumen infinitesimal y distorsiona o vulnera la ortogonalidad de los ejes principales. En ocasiones, como sucede en los sistemas caóticos, todos los ejes principales tienden a alinearse en la dirección de mayor inestabilidad, volviéndose indistinguibles los ejes principales; solo sería factible computar la tasa de convergencia o divergencia en la dirección dominante. La estimación de variaciones significativas resulta más completa sobre la base de ejes principales ortogonales, aparte de facilitarse el cómputo operacional, de ahí que la mayoría de los métodos opten por reemplazar los ejes principales regularmente, de forma que se restablezca su mutua ortogonalidad y ortonormalidad.

Los diferentes métodos que han de restituir la orientación ortogonal de los ejes principales se decantan por dos técnicas de restauración: la ortogonalización de

\footnotetext{
${ }^{4}$ Por conveniencia se usa el habitual sentido del tiempo, si bien, en términos algorítmicos, los resultados son equivalentes para una evolución temporal hacia atrás.
} 
Gram-Schmidt y la descomposición QR. El conjunto de ejes principales progresa por el atractor, fijados a la trayectoria de referencia, y deberá procederse de forma periódica a su «reortonormalización». Si el propósito es únicamente determinar si el sistema es o no caótico, con solo registrar la presencia de un exponente de Lyapunov positivo todo quedaría fácilmente confirmado. En caso de pretenderse obtener el espectro completo de exponentes de Lyapunov, no siempre las técnicas establecidas arrojan resultados favorables, puesto que es extremadamente difícil medir exponentes negativos. Las trayectorias vecinas pueden converger con el tiempo hacia direcciones normales a la dirección del flujo, donde el atractor es demasiado delgado y con escasa información. Es más, la convergencia puede manifestarse por debajo de la resolución de los datos (magnitud del ruido), por lo que resultaría muy complicado evaluar la tasa de contracción que experimentan las trayectorias vecinas. En estos casos probablemente se mediría un incorrecto exponente de Lyapunov de cero, dado que el ruido, en media, ni expande las trayectorias ni las contrae. Una posibilidad podría contemplar la evaluación de la tasa a la que trayectorias exteriores al atractor se aproximan a él, esto es, evaluar el transitorio antes de que el sistema se sumerja en el atractor; el inconveniente es que el transitorio suele ser de corta duración y en la mayoría de las sucesiones temporales ni siquiera ha sido capturado [Abarbanel et al., 1990].

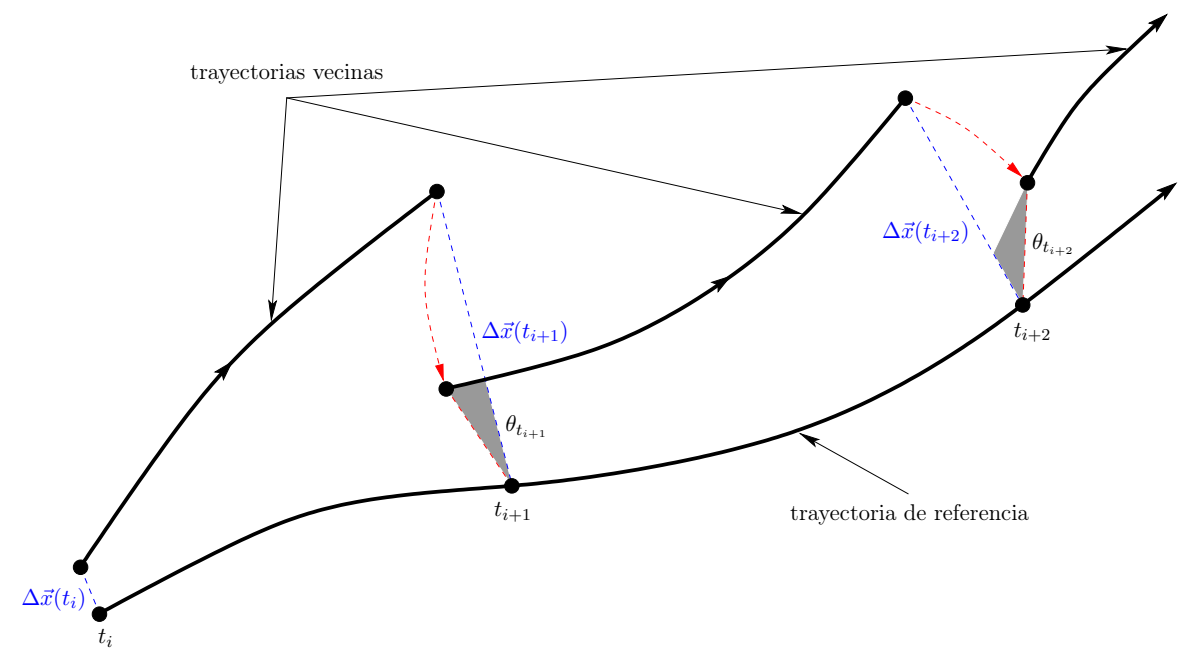

Figura 4.11: Constructo de la estimación del mayor exponente de Lyapunov a partir de la sucesión temporal. En cada instante de tiempo $t_{j}$, en que se efectúa el reemplazo de la trayectoria vecina, han de minimizarse el ángulo $\theta_{t_{j}}$ y la distancia al nuevo punto vecino inicial.

La mayoría de los métodos producen exponentes espurios que han de ser identificados y descartados [Bryant, 1992]. El número de exponentes adicionales que surgen es consecuencia del proceso de reconstrucción, ya que, por definición, la dimensión de reconstrucción es mayor que la dimensión real del sistema dinámico. Por tanto, el número de exponentes de Lyapunov, que coincide con el número de dimensiones del espacio de reconstrucción, es superior al número idóneo de dimensiones que caracterizan al sistema dinámico original; son los exponentes extras que deben desecharse. 
El método más popular toma el primer punto del espacio de reconstrucción como punto de referencia de la trayectoria de referencia [Wolf et al., 1985]. Para el punto de referencia considerado se determina un punto vecino cercano, que represente a la trayectoria vecina, de una sección temporal de los datos diferente. Durante un tiempo determinado, fijado de acuerdo con ciertos criterios prescritos de antemano, se evalúa la distancia de separación entre ambas trayectorias. El intervalo de tiempo que se emplea para medir la distancia de separación entre ambas trayectorias se extiende hasta que la distancia exceda un umbral $\varepsilon$ o hasta que otra trayectoria se aproxime. Una vez satisfecha alguna de las condiciones, se computa la pendiente local - distancia de separación final menos distancia de separación inicial, dividido por el intervalo de tiempo asociado-, procediéndose a descartar la trayectoria vecina actual. El último punto de la trayectoria de referencia, para el intervalo de tiempo considerado, se convierte en el nuevo punto de referencia, se determina un nuevo punto vecino cercano, que represente a la trayectoria vecina, y se repite el procedimiento comentado anteriormente. Así se procede hasta cubrir toda la sucesión de datos. Si se asume que la sucesión temporal abarca el atractor, se promedian los logaritmos de todas las pendientes, de acuerdo con la ecuación (4.13), a fin de obtener el mayor exponente de Lyapunov.

$$
\lambda_{\text {máx }}=\lim _{N \rightarrow \infty} \frac{1}{N} \sum_{i=1}^{N_{r}} \log \left|f^{\prime}\left(\mathbf{x}_{i}\right)\right|,
$$

donde $f^{\prime}\left(\mathbf{x}_{i}\right)$ denota la pendiente local en el estado $\mathbf{x}_{i}$.

Cuando una trayectoria vecina queda obsoleta y es reemplazada por una nueva trayectoria vecina, debe seleccionarse un nuevo punto vecino conforme a dos criterios: (1) que el punto vecino esté lo más cerca posible del punto de referencia; (2) que la orientación espacial del nuevo punto vecino conserve la orientación espacial del último punto de la trayectoria vecina abandonada (el ángulo $\theta_{t_{j}}$ de la figura 4.11 ha de ser lo más pequeño posible). Aunque el impacto de la orientación angular es menor en el cómputo de los exponentes de Lyapunov, sí ha de vigilarse una rápida divergencia de las trayectorias de referencia y vecina, pues presumiblemente una rápida divergencia está revelando trayectorias en diferentes regiones del atractor que evolucionan en diferentes direcciones debido al fenómeno del plegado (folding). El número de datos $N$ que se requieren para una acertada estimación de los exponentes de Lyapunov depende de varios factores, como el número de puntos que deben reemplazarse, el número de órbitas, alrededor del atractor, necesarias para evaluar adecuadamente el fenómeno del estiramiento (stretching), además de cómo de correcta es la reconstrucción del espacio de estados. En la práctica la cantidad de datos crece exponencialmente con la dimensión del atractor; en un caso extremo se precisa el cuadrado de la cantidad de datos necesaria para calcular la dimensión de correlación [Eckmann and Ruelle, 1992]. Sin embargo, no es tan crítico disponer de cientos de miles de datos sino cómo de recurrentes son las trayectorias en el espacio de estados.

Otro método alternativo, desarrollado independientemente en [Eckmann and Ruelle, 1985, Sano and Sawada, 1985], no considera únicamente la trayectoria vecina sino todos aquellos puntos vecinos del punto de referencia, no separados de este 
más de una distancia arbitraria $\varepsilon$. En cada paso de tiempo, suficientemente pequeño como para admitir una transformación lineal, el punto de referencia inicial, de acuerdo con las reglas que determinan la evolución dinámica del sistema, se convierte en un segundo punto de referencia. Dado que cada punto vecino presenta su particular distancia de separación, dentro del límite impuesto por $\varepsilon$, respecto al punto de referencia inicial, con cada paso de tiempo se hace un seguimiento de cuántos de los vecinos iniciales todavía se mantienen dentro de una distancia $\varepsilon$ del segundo punto de referencia, así como, para cada uno de los vecinos supervivientes, de las distancias concretas de cada uno de ellos; mediante un ajuste por regresión lineal se computa el factor por el que se multiplica la distancia de separación de los vecinos iniciales a fin de alcanzar la distancia de separación en relación con el segundo punto de referencia. Los exponentes de Lyapunov se computan, por medio de manipulaciones algebraicas, a partir de los factores multiplicativos que se obtienen a lo largo de la trayectoria de referencia. Para aplicar este método se precisa un valor de $\varepsilon$ que no sea muy pequeño, de forma que se disponga de un número suficiente de vecinos que haga más robusta la significación estadística de la prueba, reduciéndose el impacto del ruido, ni muy grande, lo que diluye la linealidad de la transformación entre puntos del espacio de estados. En la mayoría de las ocasiones se recurre a técnicas heurísticas para determinar el valor de $\varepsilon$, sin desatender que normalmente el número de vecinos de un punto de referencia cualquiera está condicionado por la cantidad de datos de la sucesión temporal, el ruido presente y la naturaleza fractal del atractor que describe la dinámica del sistema.

Los diversos métodos que se han venido proponiendo desde entonces comportan variaciones mejoradas de los modelos originales planteados [Eckmann and Ruelle, 1985, Sano and Sawada, 1985], puesto que no siempre resulta fácil descifrar fidedignamente los exponentes de Lyapunov de datos experimentales contaminados con ruido [Abarbanel et al., 1991]. En [Theiler et al., 1991], por ejemplo, se demuestra que pueden obtenerse exponentes de Lyapunov positivos de datos no caóticos. En general los diferentes métodos presentan problemas derivados de la calidad de los datos, de cómo ajustar, de forma precisa, un modelo que describa la dinámica local, y del tamaño $\varepsilon$ de la vecindad [Glass and Kaplan, 1993]. Además, los diferentes métodos no están exentos de generar exponentes de Lyapunov espurios, y, al igual que sucede con otras técnicas de análisis no lineal, introducen limitaciones en sistemas de dimensiones muy altas, debido a que las trayectorias raramente retornan a una localización previamente visitada, lo que dificulta igualmente el cálculo de las dimensiones del atractor.

\section{Métodos jacobianos}

Con objeto de operar con los métodos jacobianos en primer lugar ha de construirse un modelo que se ajuste a los datos, para, a partir de las ecuaciones del modelo, poder estimar las matrices jacobianas y, por extensión, los exponentes de Lyapunov. En el procedimiento estándar se conocen a priori las ecuaciones del sistema dinámico. En el ámbito de las sucesiones temporales las ecuaciones del sistema dinámico derivan de aproximaciones lineales, a nivel local, que modelizan la dinámica del sistema en el espacio de estados reconstruidos. Conforme al procedimiento estándar, 
las matrices jacobianas han de computarse a lo largo de la trayectoria trazada por los estados reconstruidos. En este sentido debe prestarse especial atención al hecho de que la trayectoria delineada por los estados reconstruidos refleja la dirección inestable del atractor, no la dirección transversal, caracterizada por la contracción que experimenta la dinámica, de ahí que en no pocas ocasiones resulte muy intrincado resolver los exponentes de Lyapunov negativos. Esta circunstancia se agrava por la presencia del ruido, que complica aún más el cómputo de los elementos de las matrices jacobianas, ya de por sí aproximaciones parciales.

Por otro lado, para que el atractor, y, por consiguiente, la dinámica del sistema, se despliegue completamente, la dimensión de reconstrucción $m$ debe ser mayor que la dimensión del espacio de estados original $d$. Por tanto, las matrices jacobianas suministrarían $m$ exponentes de Lyapunov, $m-d$ exponentes de Lyapunov más de los estrictamente necesarios, de ahí que se les denomine exponentes espurios o parásitos. En cualquier caso se debe proceder a su identificación y eliminación, dado que no contribuyen en nada a la caracterización de la dinámica del sistema.

Los exponentes de Lyapunov espurios pueden adoptar cualquier valor, dependiendo de factores como el esquema de aproximación que se emplee en la determinación de las matrices jacobianas, la curvatura local del atractor reconstruido, las perturbaciones (ruido) a las que se ve sometido el sistema, etc. Esto significa que los exponentes de Lyapunov pueden intercalarse entre los verdaderos exponentes, distorsionando significativamente el espectro de Lyapunov, lo que, entre otras cosas, puede conducir a conclusiones erróneas en el cálculo de la dimensión de Lyapunov (ver tabla 4.2), en razón de la conjetura de Kaplan-Yorke, detallada en la página 133. La identificación de los exponentes de Lyapunov espurios no es una tarea sencilla, salvo que se disponga de datos de muy alta calidad, en cuyo caso una técnica muy habitual comporta calcular los exponentes de Lyapunov tanto en el sentido natural del tiempo como en sentido inverso. Los exponentes de Lyapunov verdaderos experimentan un cambio de signo, dependiendo de la flecha del tiempo, a diferencia de los exponentes de Lyapunov espurios, que no ven alterados los signos de sus valores, puesto que estos exponentes espurios caracterizan direcciones en las que el atractor es muy fino [Parlitz, 1992]. En la literatura científica pueden encontrarse otras técnicas más sofisticadas para detectar y evitar los exponentes de Lyapunov espurios [Sauer et al., 1998, liu Yang et al., 2012].

Para evitar la presencia de los exponentes de Lyapunov espurios se despliega la dinámica global del sistema en un espacio de reconstrucción $m$-dimensional, a la vez que se aproxima la dinámica local (espacio tangente) por medio de un espacio $d$-dimensional, con $d \leqslant m$. Los índices de los estados vecinos de un posible estado de referencia se identifican en el espacio $m$-dimensional; con los índices fijados se reconocen los estados vecinos en el espacio $d$-dimensional, que posibilitan modelizar la dinámica local: el flujo y las matrices jacobianas [Brown et al., 1991, Bryant et al., 1990, Eckmann et al., 1986]. Las reconstrucciones local y global permiten cubrir escalas de tiempo relevantes, si bien los diferentes vectores de estado debieran abarcar una ventana temporal similar, esto es, $(m-1) \tau_{m} \approx(d-1) \tau_{d}$, siendo $\tau_{m}, \tau_{d}$ los retardos entre las componentes de los vectores de estado de los espacios $m$-dimensional y $d$ dimensional, respectivamente. Una alternativa contempla proyectar localmente, en un subespacio $d$-dimensional, el espacio de reconstrucción $m$-dimensional, mediante 
la descomposición en valores singulares de las matrices de las trayectorias locales [Stoop and Parisi, 1991].

\section{Métodos directos}

Las diferentes variantes del método directo, que posibilitan el cómputo del mayor exponente de Lyapunov, descansan en el hecho de que casi todo espacio tangente se reduce a un subespacio generado por el primer exponente de Lyapunov $\lambda_{1}$; este subespacio se ve sometido a una tasa de crecimiento asintótico conforme al valor de $\lambda_{1}$. En la práctica suele disponerse de una sucesión temporal de longitud finita, por lo que solo es posible reconstruir un número finito de estados, cuyas distancias mutuas presentan una cota inferior igualmente finita. Si un estado de referencia $\mathbf{x}_{n}$ tiene por vecino más cercano a $\mathbf{x}_{m(n)}$, los segmentos de trayectoria emergiendo de ambos estados en media divergen exponencialmente hasta que la distancia $\left\|\mathbf{x}_{m(n)+k}-\mathbf{x}_{n+k}\right\|$ exceda cierto umbral y cese la divergencia (oscilaciones) debido al tamaño o diámetro del atractor. En el método directo es crucial que se mantenga en todo momento la orientación hacia la dirección de expansión, dando por concluida la estimación de la divergencia antes de que, dependiendo del diámetro del atractor, la distancia entre los estados se sature.

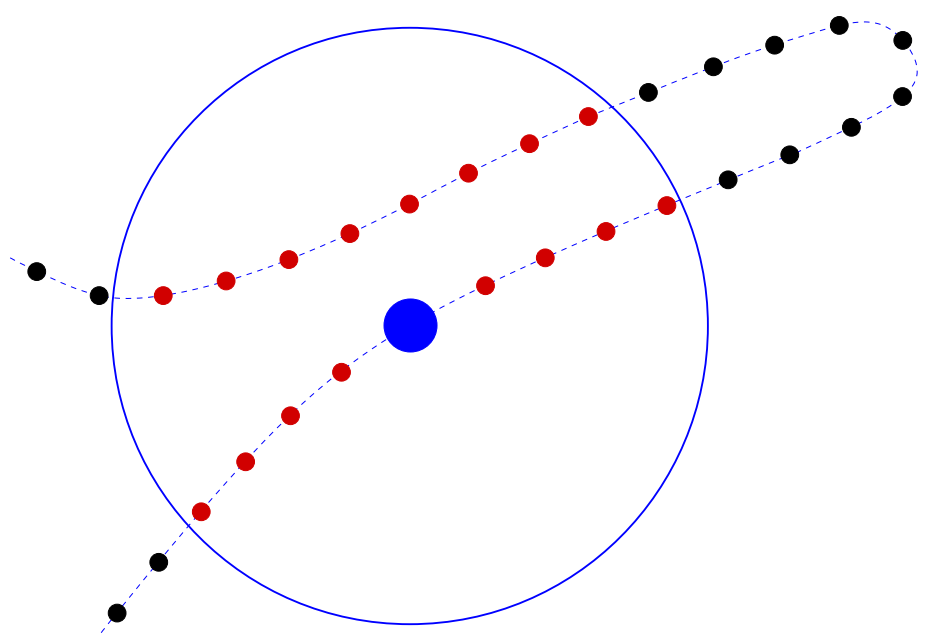

Figura 4.12: Los puntos dentro del círculo $\odot$, para una ventana temporal $w=4$, son considerados vecinos temporales del punto $\bullet$, que identifica un estado de referencia de la trayectoria de interés.

Las diversas implementaciones del método directo comparten algunos aspectos comunes [Gao and Zheng, 1993, Kantz, 1994, Rosenstein et al., 1993, Sato et al., 1987]. Sea $\mathbf{x}_{m(n)}$ el estado vecino más cercano de un estado de referencia $\mathbf{x}_{n}$, en relación con la norma euclidiana $L_{2} \cdot{ }^{5}$ Si se supone que ambos estados están separados temporalmente, esto es,

\footnotetext{
${ }^{5}$ Se admiten otras normas, como la norma máxima $L_{\infty}$ o la norma $L_{1}$. El uso de una norma concreta dependerá de la formulación estadística en que se emplee, aparte de otras consideraciones relacionadas con el tiempo de cómputo.
} 


$$
|m(n)-n|>w,
$$

siendo $w$ una escala de tiempo característica, p. ej., período medio, de la sucesión temporal. Esta separación o ventana temporal $w$, también denominada ventana de Theiler [Theiler, 1990], garantiza que los estados vecinos $\mathbf{x}_{m(n)}$ y $\mathbf{x}_{n}$ puedan considerarse condiciones iniciales de diferentes segmentos de una trayectoria, posibilitándose el cómputo del mayor exponente de Lyapunov, en términos de la tasa media de separación entre estados pertenecientes a diferentes segmentos de la trayectoria. En la figura 4.12 se ilustra un ejemplo de vecindad temporal, para una ventana $w=4$, frente a la vecindad espacial, que es la que realmente permite el cálculo de la divergencia entre trayectorias cercanas.

La separación entre estados, después de $k$ pasos de tiempo, es decir, después de un intervalo de tiempo $T=k \Delta t$, con $\Delta t$ identificando el período de muestreo, obedece a la distancia

$$
d(m(n), n, k)=\left\|\mathbf{x}_{m(n)+k}-\mathbf{x}_{n+k}\right\| .
$$

Normalmente se suele recurrir a la distancia euclidiana [Gao and Zheng, 1993, Rosenstein et al., 1993, Sato et al., 1987], o sea,

$$
d_{E}(m(n), n, k)=\left\|\mathbf{x}_{m(n)+k}-\mathbf{x}_{n+k}\right\|_{2},
$$

si bien [Kantz, 1994] apunta que es suficiente considerar la diferencia entre las últimas componentes de los estados, pues estas componentes, proyecciones de los estados reconstruidos, experimentan similar crecimiento exponencial, de acuerdo con el mayor exponente de Lyapunov, a saber,

$$
d_{U}(m(n), n, k)=\left|x\left(t_{m(n)+(m-1) \tau_{m}+k}\right)-x\left(t_{n+(m-1) \tau_{m}+k}\right)\right|,
$$

donde $\tau_{m}$ denota el retardo entre las componentes de los vectores de estado reconstruidos.

El mismo argumento esgrimido en el párrafo previo puede extenderse a la primera componente,

$$
d_{P}(m(n), n, k)=\left|x\left(t_{m(n)+k}\right)-x\left(t_{n+k}\right)\right| .
$$

Cuando la distancia $d(m(n), n, k)$ es muy pequeña, su evolución temporal puede aproximarse linealmente como

$$
d(m(n), n, k) \approx d(m(n), n, 0) e^{\hat{\lambda}_{1}(n) T}=d(m(n), n, 0) e^{\hat{\lambda}_{1}(n) k \Delta t},
$$

donde $d(m(n), n, 0)$ representa la separación inicial entre los estados vecinos $\mathbf{x}_{m(n)}$ y $\mathbf{x}_{n}$, y $\hat{\lambda}_{1}(n)$ la mayor tasa de expansión local que experimentan los segmentos de trayectoria que inician en $\mathbf{x}_{m(n)}$ y $\mathbf{x}_{n}$. Aplicando el logaritmo natural a ambos términos de la ecuación (4.19), 


$$
\begin{aligned}
\hat{\lambda}_{1}(n) & \approx \frac{1}{k \Delta t}[\ln d(m(n), n, k)-\ln d(m(n), n, 0)] \\
& =\frac{1}{k \Delta t} \ln \left(\frac{d(m(n), n, k)}{d(m(n), n, 0)}\right)
\end{aligned}
$$

Dado que la tasa de expansión no se distribuye de manera uniforme en el atractor, esta ha de promediarse a lo largo de la trayectoria disponible, seleccionándose, para cada estado de referencia $\mathbf{x}_{n}$, varios estados vecinos $\left\{\mathbf{x}_{m(n)}: m(n) \in \mathcal{U}_{n}\right\}$, donde $\mathcal{U}_{n}$ determina la vecindad de $\mathbf{x}_{n}$, que identifica un número fijo de vecinos, los $K$ vecinos más cercanos (masa fija) a $\mathbf{x}_{n}$ o el número de vecinos situados a una distancia de $\mathbf{x}_{n}$ inferior a un umbral prefijado $\varepsilon$ (tamaño fijo). Es muy habitual emplear una vecindad de masa fija, con $K=1$, esto es, sirviéndose únicamente del vecino más cercano [Gao and Zheng, 1993, Rosenstein et al., 1993, Sato et al., 1987], aunque una vecindad de tamaño fijo podría resultar más adecuada si se pretende no mezclar diferentes escalas [Kantz, 1994].

A fin de reducir la carga computacional del método directo, no se emplean todos los estados reconstruidos como estados de referencia, sino un subconjunto $\mathcal{R}$ compuesto por $N_{r}=|\mathcal{R}|$ estados, donde el símbolo | | denota la cardinalidad del subconjunto. Si $\mathcal{R}$ reúne aquellos estados de referencia con vecinos muy próximos $(d(m(n), n, 0) \rightarrow 0)$, los resultados obtenidos son muy satisfactorios. Así, con distancias logarítmicas promediadas,

$$
E(k)=\frac{1}{N_{r}} \sum_{n \in \mathcal{R}} \frac{1}{\left|\mathcal{U}_{n}\right|} \sum_{m \in \mathcal{U}_{n}} \ln d(m, n, k),
$$

donde $\left|\mathcal{U}_{n}\right|$ denota el número de vecinos del estado de referencia $\mathbf{x}_{n}$. Además,

$$
\begin{aligned}
S(k) & =E(k)-E(0) \\
& =\frac{1}{N_{r}} \sum_{n \in \mathcal{R}} \frac{1}{\left|\mathcal{U}_{n}\right|} \sum_{m \in \mathcal{U}_{n}} \ln \left(\frac{d(m, n, k)}{d(m, n, 0)}\right) .
\end{aligned}
$$

Las tasas de expansión local promediadas, conforme a la ecuación (4.20), ascienden a

$$
\begin{aligned}
\bar{\lambda}_{1} & =\frac{1}{N_{r}} \sum_{n \in \mathcal{R}} \hat{\lambda}_{1}(n) \approx \frac{1}{N_{r}} \frac{1}{k \Delta t} \sum_{n \in \mathcal{R}} \frac{1}{\left|\mathcal{U}_{n}\right|} \sum_{m \in \mathcal{U}_{n}} \ln \left(\frac{d(m, n, k)}{d(m, n, 0)}\right) \\
& =\frac{1}{k \Delta t} S(k)=\frac{1}{k \Delta t}[E(k)-E(0)] .
\end{aligned}
$$

De las ecuaciones (4.22) y (4.23) se deduce que

$$
S(k) \approx k \Delta t \bar{\lambda}_{1},
$$




$$
E(k) \approx k \Delta t \bar{\lambda}_{1}+E(0) .
$$

En las ecuaciones (4.21), (4.22), (4.23) y (4.25) puede reemplazarse $E(k)$ por $E_{E}(k), E_{P}(k)$ o $E_{U}(k)$, en función de la distancia $d_{E}, d_{P}$ o $d_{U}$, respectivamente, que se use en la ecuación (4.21).

Gráficamente, la estimación del mayor exponente de Lyapunov comprende la pendiente del segmento lineal que se obtiene representando $S(k)$ vs. $k \Delta t$, o bien $E(k)$ vs. $k \Delta t$ si en las ecuaciones (4.20) y (4.22) se suprime el factor de normalización que repara en la distancia de separación inicial $d(m, n, 0)$. Ambas representaciones son equivalentes, pues únicamente difieren en un factor constante de desplazamiento $E(0)$. Otra forma alternativa, sugerida en [Sato et al., 1987], considera

$$
\bar{\lambda}_{1} \approx \frac{E(k+l)-E(k)}{l \Delta t}=\frac{S(k+l)-S(k)}{l \Delta t},
$$

con objeto de identificar un aplanamiento en la gráfica que representa $E(k+l)-E(k)$ vs. $k$, para algún rango de valores de $k$, concretamente en aquel rango en que se delimita un segmento lineal de acuerdo con el procedimiento descrito unas líneas más arriba. Este procedimiento constituye una aproximación por diferencias finitas de la pendiente de $E(k)$ vs. $k \Delta t$, prestando especial atención al intervalo $l \Delta t$; los mejores resultados se obtienen cuando el intervalo $l \Delta t$ es suficientemente largo, si bien no debe rebasarse la región lineal, a priori desconocida, en la que las distancias crecen exponencialmente.

En [Wolf et al., 1985] se propone un método para estimar el mayor exponente de Lyapunov que evita la saturación de las distancias entre los estados de referencia $\mathbf{x}_{n}$ y los estados vecinos $\mathbf{x}_{m(n)}$, debido al plegado no lineal. El planteamiento del método precisa monitorizar, conforme avanza el tiempo, la distancia entre el estado de referencia $\mathbf{x}_{n}$ y el estado vecino $\mathbf{x}_{m(n)}$ hasta que se sobrepase un umbral prefijado; una vez alcanzado el umbral se reemplaza el estado vecino inicial $\mathbf{x}_{m(n)}$ por otro estado vecino $\mathbf{x}_{m^{\prime}(n)}$ más próximo y, en la medida de lo posible, con idéntica orientación, de forma que se preserve la dirección local, correspondiente al mayor exponente de Lyapunov. Con esta estrategia también es factible, no sin gran dificultad, computar el segundo mayor exponente de Lyapunov. El método se antoja robusto en el análisis de datos experimentales fisiológicos [Fell et al., 1993], si bien uno de los inconvenientes de este método estriba en que, cuando el algoritmo se enfrenta a sucesiones temporales estocásticas, puede suministrar exponentes de Lyapunov poco convincentes, en función del prefiltrado al que se someta la sucesión temporal [Dämmig and Mitschke, 1993]. Otra desventaja, similar a la que se aprecia en los métodos jacobianos, y que apela a la prudencia y cautela en la explicación del fenómeno observado, radica en que no se dispone de capacidad para verificar si en los resultados recabados por el algoritmo subyace realmente un crecimiento exponencial, es decir, aun careciéndose de una sucesión temporal en la que no es viable cuantificar una divergencia exponencial, el algoritmo proporciona un valor para el mayor exponente de Lyapunov, pudiendo llevar a una malinterpretación del comportamiento dinámico de la sucesión temporal bajo estudio. 


\subsubsection{Dimensiones}

No existe una única noción de dimensión, si bien en el espacio euclidiano tradicional el concepto de dimensión no admite duda alguna, un punto en el espacio se caracteriza por sus coordenadas (dirección vectorial), esto es, un subconjunto de un espacio euclidiano tiene asociado una dimensión topológica: los puntos aislados presentan dimensión cero; las curvas tienen dimensión uno; las superficies planas dimensión dos; y los sólidos dimensión tres [Hurewicz and Wallman, 1941, Engelking, 1978]. Si se abandona la geometría elemental aparecen subconjuntos que no se identifican claramente con estas categorías. En este sentido las matemáticas han definido una noción más general del término dimensión, de forma que, desde diversas perspectivas y para diferentes propósitos, se abarque la descripción de todos los posibles subconjuntos.

Desde un punto de vista geométrico un objeto se caracteriza por su volumen (capacidad), lo que, en el caso de objetos no regulares, ha derivado en el concepto de dimensión fractal; una dimensión fractal admite valores no enteros o fraccionarios para la dimensión del objeto [Mandelbrot, 1983]. En un territorio aún más extraño, como los sistemas caóticos y los atractores extraños, la dimensión prescinde de la geometría pura, y se adentra en el ámbito de la probabilidad (medición). El conocimiento del futuro se limita a la información contenida en la distribución de probabilidad de los puntos que conforman el atractor. Si se asigna una dimensión a la distribución de probabilidad, la dimensión de información proporciona una noción de dimensión de carácter probabilístico.

$\mathrm{Al}$ igual que un conjunto es fractal si su dimensión fractal excede a su dimensión topológica, una distribución de probabilidad se denomina medida fractal si su dimensión fractal excede a su dimensión de información [Farmer, 1982].

Dado un conjunto $C$, que identifica el atractor, se define una partición uniforme $\beta=\left\{B_{i}\right\}$ del espacio de estados que cubra $C$, de acuerdo con una escala de resolución (factor de escala) o graduación del instrumento de medida $\varepsilon$. Efectuando repetidas mediciones a intervalos de tiempo aleatorios, resulta factible trazar un histograma de las frecuencias de ocurrencia $P_{i}$ de cada $i$-ésimo elemento de la partición. El conjunto de probabilidades $\left\{P_{i}\right\}$ determina una distribución de probabilidad asintótica de grano grueso (coarse grained asymptotic probability distribution). En este sentido se define una medida

$$
\bar{\mu}(C)=\sum_{i} P_{i} .
$$

En el límite inferior de la escala de resolución $(\varepsilon \rightarrow 0)$ y para infinitas muestras, $P_{i}=\mu\left(B_{i}\right)$.

El comportamiento dinámico del caos se manifiesta en la dependencia sensible de su evolución temporal a las condiciones iniciales. El comportamiento temporal extraño de un sistema caótico tiene su homólogo geométrico en la estructura de su atractor. La inestabilidad consustancial a todo movimiento caótico lleva aparejada un crecimiento exponencial de distancias infinitesimales. Si se considera una región infinitesimal del atractor como un subconjunto de condiciones iniciales, con el transcurso del tiempo la función de evolución del sistema distribuirá las diferentes 
imágenes de las condiciones iniciales por todo el atractor. Esta propiedad, denominada mixing, es la responsable del decaimiento exponencial que experimenta la función de autocorrelación. Una consecuencia inmediata comporta que un atractor típico ha de ser continuo a lo largo de las direcciones inestables.

Por un lado, cualquier estructura a pequeña escala, debido a la función de estiramiento (stretching), crece hasta alcanzar un tamaño similar al del propio atractor, lo que contradiría la pretendida invariancia. Por otro lado, debido a la disipación, existen direcciones del espacio en las que el atractor se contrae (dirección estable). Para estas direcciones resulta factible aplicar similar razonamiento que en el caso de direcciones inestables, aun extrayéndose diferentes conclusiones. Sean dos condiciones iniciales muy próximas entre sí; si el vector diferencia es colineal a la dirección estable, esta diferencia se reduce con el tiempo hasta finalmente desaparecer. Por tanto, el atractor, en el límite asintótico, es 0-dimensional en las direcciones estables. Aun en el caso de que esto último pudiera suceder, no es infrecuente observar una estructura más complicada, dado que en la argumentación previa se ignora el proceso de plegado (folding), resultado de la no linealidad del sistema dinámico. De este modo, para disponer de un atractor finito y, al mismo tiempo, de una divergencia exponencial de las condiciones iniciales, este se pliega en el espacio y finalmente se mapea sobre sí mismo. Este proceso parcialmente «rellena» la dirección estable, obrando, junto con la disipación, la autosemejanza y la invariancia con la escala del subconjunto invariante. Si una parte del atractor extraño se amplía se obtiene una réplica similar del total. Por consiguiente, el atractor muestra una estructura autosemejante a cualquier escala en términos estadísticos.

Las diferentes definiciones de dimensión atienden al escalamiento que experimenta una magnitud física contenida en un elemento infinitesimal de volumen, véanse su volumen, su masa, la información, etc., con su tamaño, o al número de grados de libertad presentes en un sistema físico.

Una concepción geométrica de dimensión corresponde al escalamiento de una magnitud física, contenida en un elemento infinitesimal de volumen, con el tamaño del mismo, esto es,

$$
\text { magnitud } \sim \text { med }^{\text {dimensión }},
$$

donde la magnitud puede identificar volumen, masa, información, etc., y la medida geométrica med una distancia lineal, como la longitud del lado de un hipercubo. Por tanto, conforme a la ecuación (4.28), la dimensión se expresaría como

$$
\text { dimensión }=\lim _{\text {med } \rightarrow 0} \frac{\log \text { magnitud }}{\log \text { med }},
$$

con un límite infinitesimal en la medida geométrica para asegurar invariancia en la escala ante transformaciones de coordenadas infinitamente continuas. Además, el límite infinitesimal sugiere una estimación local de la dimensión, por lo que cualquier definición global de dimensión requiere un promediado.

La forma más obvia de cuantificar el tamaño de un conjunto $B$ pasa por especificar su radio o su diámetro, esto es,

$$
\operatorname{diám}(B)=\sup \{\|\mathbf{x}-\mathbf{y}\|: \mathbf{x}, \mathbf{y} \in B\},
$$


donde $\|\mathbf{x}-\mathbf{y}\|$ representa la distancia entre $\mathbf{x}$ e $\mathbf{y}$. Esta distancia depende de la norma utilizada en el espacio métrico. Si $\mathbf{x}, \mathbf{y} \in \mathbb{R}^{d}$, la norma vectorial $L_{p}$ asciende a

$$
\|\mathbf{x}-\mathbf{y}\|=\left(\sum_{i=1}^{d}\left|x_{i}-y_{i}\right|^{p}\right)^{1 / p}
$$

con $|\cdot|$ simbolizando el valor absoluto. La dimensión fractal es invariante frente a la norma escogida, si bien la norma $L_{\infty}$ es la más fácil de computar y la norma euclídea $L_{2}$ no se ve afectada por las rotaciones de los puntos.

Sea un sistema dinámico discreto o mapa (tiempo discreto, $n$ )

$$
\mathbf{x}_{n+1}=F\left(\mathbf{x}_{n}\right)
$$

o un sistema dinámico continuo o ecuación diferencial ordinaria (tiempo continuo, $t$ )

$$
\frac{\mathrm{d} \mathbf{x}(t)}{\mathrm{d} t}=f(\mathbf{x}(t))
$$

con $\mathbf{x} \in \mathbb{R}^{d}$ siendo un vector de estado. En general un atractor es algo que atrae condiciones iniciales, una vez finalizado el transitorio. Más precisamente, un atractor es un subconjunto compacto $A$, de forma que el subconjunto límite ${ }^{6}$ de una órbita es $A$. La cuenca de atracción de $A$ comprende la cerradura del subconjunto de condiciones iniciales que se aproximan a $A$.

La dimensión permite caracterizar las propiedades de un atractor; proporciona la cantidad de información necesaria para identificar, con cierto grado de precisión, la posición de un punto del atractor. De otra parte, caracteriza el número mínimo de variables esenciales para modelizar la dinámica del atractor.

En el caso de atractores simples, con una estructura regular, la dimensión admite valores enteros. En el caso de atractores caóticos (extraños), no catalogados como variedades, su naturaleza errática e irregular (fracturada) induce una dimensión no entera. En el reconocimiento de las propiedades de un atractor caótico debe considerarse no solo el atractor en sí mismo, sino también la distribución o densidad de puntos del atractor, para lo que se define la medida natural de un atractor, que procura la frecuencia relativa con la que una órbita visita diferentes regiones del atractor, esto es, la medida natural de una región es proporcional a la frecuencia con la que es visitada (nociones probabilísticas).

Las definiciones más significativas de dimensión engloban dos tipos [Farmer et al., 1983]: las que contemplan propiedades métricas (concepto de distancia), y las que dependen de propiedades métricas y probabilísticas (medida natural del atractor). Típicamente, las dimensiones métricas o dimensiones fractales, $d_{F}$, adquieren el mismo valor, al igual que las dimensiones probabilísticas o dimensiones de la medida natural, $d_{\mu}$, si bien las últimas presentan un valor menor $\left(d_{\mu}<d_{F}\right)$. La dimensión de la medida natural se obtiene a partir de las propiedades de estabilidad de una órbita del atractor (números de Lyapunov). La dimensión de Lyapunov pertenece a otra categoría aparte, dado que se evalúa en términos de las propiedades dinámicas del atractor.

\footnotetext{
${ }^{6}$ El subconjunto límite caracteriza el estado de un sistema dinámico cuando $t \rightarrow \infty$ o $n \rightarrow \infty$.
} 


\subsubsection{Clasificación de las dimensiones}

La clasificación de los diferentes tipos de dimensiones fractales permite identificar qué condiciones determinan cuándo las dimensiones fractales pueden o no coincidir. En los fractales estrictamente autosemejantes (monofractales) todas las dimensiones fractales son equiparables, dentro del nivel de exactitud impuesto por la precisión computacional. Un monofractal presenta idéntica complejidad en todo el espacio que ocupa. Por el contrario, en los multifractales, mezcla de monofractales, las diversas dimensiones fractales difieren, reflejando una mayor complejidad subyacente. En este sentido el espectro de dimensiones de Rényi posibilita extraer cada objeto fractal del conjunto multifractal, ${ }^{7}$ contribuyendo a una completa caracterización física del mismo [Hentschel and Procaccia, 1983]. El espectro de dimensiones de Rényi encierra un amplio abanico de dimensiones fractales, las morfológicas, las basadas en la entropía, o contenido de información, y las basadas en transformaciones, sin olvidar que implícitamente pueden catalogarse las dimensiones fractales conforme al algoritmo de cómputo, así como a su aplicabilidad a ciertos procesos físicos [Kinsner, 2005].

Las dimensiones morfológicas atienden a aspectos puramente geométricos, como la forma o estructura que adopta el objeto fractal. Este tipo de dimensiones se aplica a objetos cuya distribución de la medida, como la distribución probabilística, es homogénea (uniforme) o se desconoce a priori. Las dimensiones basadas en la entropía consideran una medida de probabilidad o una medida de correlación en el objeto fractal, en términos de la frecuencia con la que una región del fractal es visitada por una trayectoria (fractal temporal) o en términos de la distribución de la medida (fractal espacial). En un fractal temporal se examina la evolución temporal del sistema dinámico; en un fractal espacial la hetereogeneidad espacial de los estados que conforman la trayectoria. Finalmente, las dimensiones basadas en transformaciones analizan el objeto fractal desde diferentes dominios, como, por ejemplo, el dominio frecuencial, a fin de extraer propiedades del fractal encubiertas en su dominio natural, el dominio temporal. En cualquier caso la exactitud con la que se computa la dimensión fractal de un objeto, la saturación que experimenta su estimación en los límites fronterizos, así como problemas derivados de su representación en presencia de un número limitado de datos, entre otras cuestiones, no han de tratarse a la ligera. En la tabla 4.2 se extracta una taxonomía de las principales concepciones de dimensión, en el contexto de sucesiones temporales que caracterizan la dinámica de un presumible sistema físico complejo.

\footnotetext{
${ }^{7}$ El espectro multifractal o espectro de singularidad de Mandelbrot desempeña idéntico cometido [Mandelbrot, 1988].
} 
Tabla 4.2: Taxonomía de las dimensiones.

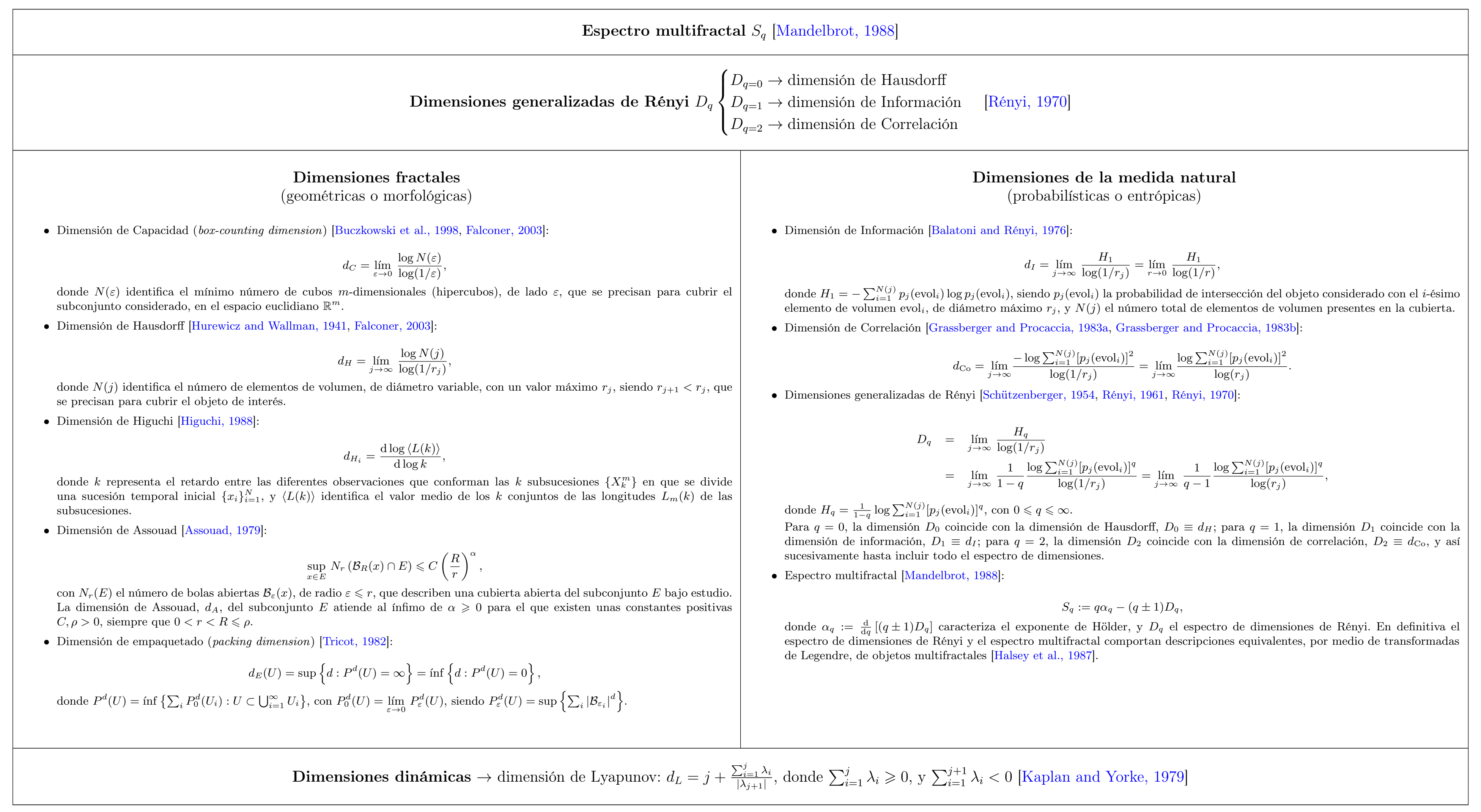




\subsubsection{Dimensión fractal en sucesiones temporales}

Los fractales son objetos rugosos que oponen resistencia a cualquier medida convencional, como la longitud, el área o el volumen. Pueden clasificarse como objetos sólidos, en el sentido de que pueden encontrarse en la naturaleza, véanse nubes o helechos, o como atractores extraños [Theiler, 1990]. Estos comprenden objetos conceptuales que existen en el espacio de estados de sistemas dinámicos caóticos. Los objetos fractales contienen estructuras anidadas, al igual que en las populares $m a-$ trioskas. La naturaleza autosemejante de las estructuras, su invariancia con la escala, es quizás el agente inductor que mejor caracteriza la belleza de la mayoría de los fractales.

Un conjunto es autosemejante en sentido estricto si puede expresarse como una unión de subconjuntos en los que cada uno de ellos es una copia reducida (geométricamente similar) del conjunto total. En la mayoría de los fractales el anidamiento de subconjuntos no es perfecto, y una versión amplificada de un subconjunto no reproduce exactamente el conjunto entero. En este caso los fractales son autosemejantes en sentido estadístico, esto es, guardan una apariencia cualitativa no geométricamente similar. Un fractal es un conjunto autoafín si puede descomponerse en subconjuntos que admiten un mapeo lineal al conjunto total. Si el mapeo lineal implica únicamente rotaciones, traslaciones y escalamiento uniforme (dilatación isotrópica), el fractal es considerado autosemejante. En caso de que los escalamientos no sean uniformes (dilataciones anisotrópicas), el fractal es considerado autoafín. En definitiva los fractales autosemejantes conforman un subconjunto de los fractales autoafines.

El caos identifica el comportamiento irregular, omnipresente en la naturaleza, de ecuaciones simples. Estas ecuaciones simples obedecen a las leyes newtonianas. Normalmente la complejidad de un sistema físico se asocia al número de grados de libertad que presenta el sistema. Los grados de libertad pueden ser nominales o efectivos; los nominales comportan los posibles grados de libertad mientras que los efectivos se refieren a los que realmente caracterizan la dinámica del sistema. Los grados de libertad insinúan un comportamiento colectivo, que, bajo el calificativo de autoorganización, ha recibido especial atención en los últimos años [Bak, 1996]. Esta cualidad de muchos sistemas físicos desafía en principio a las leyes de la termodinámica, puesto que los sistemas autoorganizados reducen aparentemente su propia entropía mediante transferencia disipativa hacia el medio circundante. La autoorganización surge en sistemas dinámicos disipativos cuando, una vez transcurrido el período transitorio, el número de grados de libertad, en relación con los nominales, se reduce drásticamente, esto es, el sistema es atraído hacia una región del espacio de estados de dimensión más baja. Esta dimensión identifica los grados de libertad efectivos de la dinámica actual del sistema. Un sistema nominalmente complejo ha modificado su condición, se ha autoorganizado, y se ha convertido en un sistema caótico de baja dimensión. La habilidad para discernir entre un comportamiento irregular de baja dimensión y una dinámica errática de alta dimensión (proceso estocástico) constituye la piedra angular que fomentó, desde sus comienzos, el estudio de los sistemas caóticos. 


\section{Atractores extraños}

Los atractores extraños revelan una dinámica no lineal de un sistema físico. En términos físicos un sistema dinámico es todo aquello que evoluciona con el tiempo. En términos matemáticos un sistema dinámico queda definido por su espacio de estados $\mathbb{R}^{d}$, que describe las $d$ variables que caracterizan cada posible estado del sistema, y por la dinámica física del sistema, el operador de evolución $\phi_{t}$, que determina cómo cambia cada estado del sistema con el tiempo $t$. Un elemento $\mathbf{x} \in \mathbb{R}^{d}$ especifica el estado actual del sistema, siendo $d$ el número de grados de libertad. El operador de evolución $\phi_{t}$ comprende una familia de funciones $\phi_{t}: \mathbb{R}^{d} \rightarrow \mathbb{R}^{d}$ que mapean el estado actual del sistema a un estado futuro, $t$ unidades de tiempo más tarde. El operador de evolución satisface dos reglas básicas:

1. $\phi_{0}(\mathbf{x})=\mathbf{x}$,

2. $\phi_{t_{2}+t_{1}}(\mathbf{x})=\phi_{t_{2}}\left(\phi_{t_{1}}(\mathbf{x})\right)$.

Además, para asegurar que la dinámica sea no lineal $\phi_{t}\left(a_{1} \mathbf{x}_{1}+a_{2} \mathbf{x}_{2}\right) \neq a_{1} \phi_{t}\left(\mathbf{x}_{1}\right)+$ $a_{2} \phi_{t}\left(\mathbf{x}_{2}\right)$. El operador de evolución $\phi_{t}$ puede identificar un mapa discreto o un sistema de ecuaciones diferenciales ordinarias. ${ }^{8}$ Los sistemas disipativos merecen especial consideración en la teoría de los sistemas dinámicos porque, a diferencia de los sistemas conservativos, incluyen a los atractores extraños entre sus diferentes opciones dinámicas. En un sistema disipativo todo elemento infinitesimal de volumen del espacio de estados tiende a cero cuando $t \rightarrow \infty$, esto es, si $B \subset \mathbb{R}^{d}$, con medida de Lebesgue (volumen) $\mu_{L}(B)$, entonces

$$
\lim _{t \rightarrow \infty} \mu_{L}\left(\phi_{t}(B)\right)=0
$$

con

$$
\mu_{L}\left(\phi_{t}(B)\right) \sim \mu_{L}(B) e^{-\lambda t},
$$

siendo $\lambda$ la tasa de disipación media (exponencial). Un atractor $A$ de un sistema dinámico comprende el subconjunto del espacio de estados al que tiende la evolución del sistema. Toda condición inicial $\mathbf{x}_{0}$ en la vecindad del atractor se aproxima $\phi_{t}\left(\mathbf{x}_{0}\right)$ arbitrariamente cerca al atractor $A$ cuando $t \rightarrow \infty$. Si el atractor $A$ es fractal se dice que es extraño.

\section{Medida natural invariante}

Dado que el elemento infinitesimal de volumen de la ecuación (4.32) se hace cero, cuando $t \rightarrow \infty$, se requiere otra medida $\mu$ que permita cuantificar cómo se desarrolla la dinámica del sistema dentro del atractor $A$. Para definir una medida $\mu$ en el conjunto $A$ es preciso asociar a cada posible subconjunto de $A$ una medida $\mu(U)$ que cuantifique de alguna manera cuánto del conjunto $A$ está contenido en el subconjunto $U$, con $U \subset A$. La medida refleja la densidad del subconjunto $U$,

\footnotetext{
${ }^{8}$ Las ecuaciones diferenciales parciales y las ecuaciones diferenciales con retardo presentan una dimensión $d=\infty$.
} 
caracterizando de algún modo su contribución a la masa de $A$. Se dice que la medida $\mu$ es invariante con la evolución temporal si

$$
\mu(U)=\mu\left(\phi_{-t}(U)\right)
$$

donde

$$
\phi_{-t}(U)=\left\{\mathbf{x} \in \mathbb{R}^{d}: \phi_{t}(\mathbf{x}) \in U\right\} .
$$

Desde una perspectiva física, la medida natural ha de contabilizar qué proporción de tiempo invierte una trayectoria típica en cada región del atractor, o el porcentaje de veces que se visita. Por tanto, una formulación de la medida natural atiende a

$$
\mu(U)=\lim _{T \rightarrow \infty} \frac{1}{T} \int_{0}^{T} \mathbf{1}_{U}\left(\phi_{t}\left(\mathbf{x}_{0}\right)\right) \mathrm{d} t,
$$

donde $\mathbf{1}_{U}$ identifica la función indicatriz o característica de $U$, y $\mathbf{x}_{0}$ una condición inicial representativa.

\section{Sensibilidad a las condiciones iniciales}

El sello distintivo del caos es su dependencia sensible de las condiciones iniciales. Esta sensibilidad a las condiciones iniciales es revelada por los exponentes de Lyapunov y por la entropía de Kolmogórov-Sinái (para más información sobre esta entropía remítase a 4.2.3.2). Los exponentes de Lyapunov evalúan la tasa exponencial de divergencia de trayectorias vecinas, mientras que la entropía de Kolmogórov-Sinái estima el flujo de información del sistema dinámico, esto es, el ritmo promedio al que el sistema crea información. Si se conoce el estado $\mathbf{x}(t)$ del sistema en el instante de tiempo $t$ con una exactitud (incertidumbre) $\varepsilon$, el estado futuro, transcurrido un intervalo de tiempo $\Delta t$, obedece a $\mathbf{x}(t+\Delta t)=\phi_{\Delta t}(\mathbf{x}(t))$, con una incertidumbre presumiblemente mayor que $\varepsilon$. En caso de pretender conocer el estado del sistema en el instante $t+\Delta t$ con una exactitud $\varepsilon$ igual a la establecida para el estado $\mathbf{x}(t)$, en el instante de tiempo $t$, se precisa disponer de nueva información; el sistema está de algún modo creando información. ${ }^{9}$

La predicciones a largo plazo de un sistema caótico son a priori imposibles, aun conociéndose el operador de evolución $\phi_{t}$, debido a que errores de medición de las condiciones iniciales se propagan muy rápidamente, a un ritmo exponencial. Sean

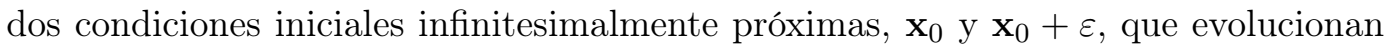
con el tiempo, de acuerdo con la expansión en series de Taylor,

$$
\phi_{t}\left(\mathbf{x}_{0}+\varepsilon\right)=\phi_{t}\left(\mathbf{x}_{0}\right)+\mathbf{J}_{\phi_{t}}\left(\mathbf{x}_{0}\right) \varepsilon+O\left(|\varepsilon|^{2}\right),
$$

donde $\mathbf{J}_{\phi_{t}}\left(\mathbf{x}_{0}\right)$ representa la matriz jacobiana, que permite la linealización de $\phi_{t}$ en torno a $\mathbf{x}_{0}$, es decir,

$$
\mathbf{J}_{\phi_{t}}\left(\mathbf{x}_{0}\right)=\frac{\partial \phi_{t}\left(\mathbf{x}_{0}\right)}{\partial \mathbf{x}_{0}} .
$$

\footnotetext{
${ }^{9}$ Igualmente se puede decir que el sistema ha perdido información, dado que la exactitud con la que se definió el estado $\mathbf{x}(t)$ en el instante $t$ se ha perdido.
} 
Los elementos de la matriz jacobiana $\mathbf{J}_{\phi_{t}}\left(\mathbf{x}_{0}\right)$ obedecen a

$$
\left(\mathbf{J}_{\phi_{t}}\left(\mathbf{x}_{0}\right)\right)_{i j}=\frac{\Delta x_{0}^{(i)}(t)}{\Delta x_{0}^{(j)}(0)},
$$

donde $x_{0}^{(i)}(t)$ identifica la $i$-ésima componente del vector de estado $\mathbf{x}_{0}$ en el instante de tiempo $t$. Por tanto, una distancia de separación inicial $\varepsilon$ entre las condiciones iniciales se ve modificada por $\mathbf{J}_{\phi_{t}} \varepsilon$. En el caso de sistemas disipativos el jacobiano determina la contracción que experimenta con el tiempo un elemento de volumen del espacio de estados, y los autovalores de la matriz jacobiana especifican la divergencia temporal de trayectorias vecinas. Los exponentes de Lyapunov cuantifican la tasa media de expansión de estos autovalores, a saber,

$$
\lambda_{i}=\lim _{t \rightarrow \infty} \frac{1}{t} \log \mid i \text {-ésimo autovalor de } \mathbf{J}_{\phi_{t}} \mid, \quad i=1, \ldots, d .
$$

Por convención, los exponentes de Lyapunov se ordenan en sentido descendente, $\lambda_{1} \geqslant \lambda_{2} \geqslant \cdots \geqslant \lambda_{d}$. El mayor exponente de Lyapunov es clave en la dinámica de un sistema físico; si este es positivo la divergencia predominará para el autovector (autodirección) asociado, asegurándose en principio un comportamiento caótico. En cualquier otro caso las trayectorias concurren a un punto fijo, un ciclo límite o un toroide. En sistemas disipativos la suma de los exponentes de Lyapunov es negativa, $\lambda_{1}+\lambda_{2}+\cdots+\lambda_{d}=-\Lambda$, siendo $\Lambda$ la tasa media de contracción que experimentaría todo elemento infinitesimal de volumen. La suma de los exponentes de Lyapunov positivos identifica la entropía de Kolmogórov-Sinái; en otras palabras, la suma de los exponentes de Lyapunov positivos coincide con la tasa media de creación (pérdida) de información.

\subsubsection{Algoritmos para computar la dimensión}

A partir de una sucesión temporal de longitud finita, que podría reproducir la trayectoria de un sistema dinámico, ha de recurrirse a métodos numéricos para computar la dimensión fractal del atractor subyacente, no de la trayectoria disponible. En una primera aproximación la dimensión de capacidad se aviene a una implementación algorítmica relativamente sencilla, si bien adolece de limitaciones prácticas, particularmente cuando la dimensión de reconstrucción es alta [Greenside et al., 1982].

El método más popularmente extendido para estimar la dimensión fractal es el algoritmo de correlación, fundamentado en un estadístico que responde a la distancia entre pares de puntos. Para el cómputo de la dimensión fractal, sobre la base del algoritmo de correlación, se puede optar por un criterio de tamaño fijo, en el que se computa el número de vecinos situados dentro de un radio de acción prefijado y constante (todas las celdas son del mismo tamaño), o por un criterio de masa fija, en que el número de vecinos por punto es fijo (las celdas son de diferente tamaño).

Se han ideado diversos métodos que observan las propiedades dinámicas del atractor, dado que muchas aplicaciones actuales pretenden predecir comportamientos futuros; en el contexto del cálculo de las dimensiones, su importancia radica en que el índice de acierto de un mecanismo de predicción está relacionado con el determinismo subyacente y con una presumible baja dimensión. Por otro lado, la conjetura de 
Kaplan-Yorke permite establecer relaciones entre las dimensiones y los exponentes de Lyapunov. Además, se introduce la noción de dimensión intrínseca, una dimensión entera que identifica la variedad de dimensión más baja capaz de contener los datos disponibles. La dimensión intrínseca comporta una cota superior para la dimensión fractal.

Normalmente, los algoritmos planteados facilitan el cómputo de la dimensión generalizada, lo que permite cuantificar la naturaleza multifractal de un atractor. No obstante, no en pocas ocasiones lo que se pretende no es tanto asignar un número concreto a una dimensión como conocer si el número de grados de libertad es grande o pequeño, esto es, el objetivo no es tanto proporcionar una estimación precisa de la dimensión como sí discernir entre ruido o caos a un bajo coste computacional, máxime cuando los datos experimentales suelen ser escasos y con mucho ruido imbricado.

Una posible clasificación de los algoritmos que permiten estimar la dimensión fractal de un atractor descansa sobre dos criterios: tamaño fijo o masa fija. Los algoritmos de tamaño fijo dividen el espacio en celdas de igual tamaño y los algoritmos de masa fija dividen el espacio en celdas de tamaño variable, de forma que cada celda contenga el mismo número de puntos.

\section{Algoritmos de tamaño fijo}

La función de masa puntual $\mu\left(\mathcal{B}_{r}(\mathbf{x})\right)$ caracteriza la densidad de puntos encerrados en una bola $\mathcal{B}_{r}(\mathbf{x})$, una bola de radio $r$ centrada en el punto x. Cómo escala esta densidad con el radio $r$ queda definido por la dimensión puntual $d_{\text {puntual }}$. Si el escalamiento coincide en todos los puntos $\mathbf{x}$ del atractor, el fractal es uniforme, $\mathbf{y}$, por consiguiente, las dimensiones generalizadas $D_{q}$ son constantes e independientes de $q$. Sin embargo, la dimensión puntual no suele ser igual para todos los puntos del atractor, de ahí que se adquiera una muestra de puntos del atractor, se compute su dimensión puntual, según la ecuación (4.41), y se aplique algún promedio que represente lo más fielmente la dimensión global del atractor.

$$
d_{\text {puntual }}(\mathbf{x})=\lim _{r \rightarrow 0} \frac{\log \mu\left(\mathcal{B}_{r}(\mathbf{x})\right)}{\log r} .
$$

La estrategia que mejores resultados arroja en términos de promedio puntual es la integral de correlación $C(r)$, introducida por Peter Grassberger e Itamar Procaccia [Grassberger and Procaccia, 1983b]. De esta forma,

$$
C(r)=\left\langle\mu\left(\mathcal{B}_{r}(\mathbf{x})\right)\right\rangle,
$$

donde $\mu\left(\mathcal{B}_{r}(\mathbf{x})\right)$, para una sucesión temporal finita $\left\{\mathbf{x}_{i}\right\}_{i=1}^{N}$ de $N$ vectores de estado reconstruidos, puede aproximarse como

$$
\begin{aligned}
\mu\left(\mathcal{B}_{r}(\mathbf{x})\right) & \approx \frac{\#\left\{\mathbf{x}_{i}: i \neq j \wedge\left\|\mathbf{x}_{i}-\mathbf{x}_{j}\right\| \leqslant r\right\}}{N-1} \\
& =\frac{1}{N-1} \sum_{\substack{i=1 \\
i \neq j}}^{N} \Theta\left(r-\left\|\mathbf{x}_{i}-\mathbf{x}_{j}\right\|\right)
\end{aligned}
$$


donde $\Theta$ identifica la función escalón de Heaviside, esto es, $\Theta(x)=0$, para $x<0$ y $\Theta(x)=1$ para $x \geqslant 0$. Un detalle importante, con objeto de no subestimar el cálculo, implica excluir $i=j$ [Grassberger, 1988] y todos los valores de $i$ tal que $|i-j|<W$, siendo $W$ la denominada ventana de Theiler [Theiler, 1986].

De la ecuación (4.42) se deriva la dimensión de correlación $d_{\mathrm{Co}}$, esto es,

$$
d_{\mathrm{Co}}=\lim _{r \rightarrow 0} \frac{\log C(r)}{\log r} .
$$

Si se reformula $C(r)$ para adaptar su cómputo a una sucesión finita de datos,

$$
\begin{aligned}
C(N, r) & =\frac{\# \text { de distancias menores que } r}{\# \text { de distancias totales }} \\
& =\sum_{i=1}^{N} \mu\left(\mathcal{B}_{r}\left(\mathbf{x}_{i}\right)\right) \\
& =\frac{1}{N(N-1)} \sum_{i \neq j} \Theta\left(r-\left\|\mathbf{x}_{i}-\mathbf{x}_{j}\right\|\right) .
\end{aligned}
$$

El rango dinámico de $C(N, r)$ opera entre un mínimo igual a 1 a un máximo igual a $2 / N^{2}$, muy superior al que exhiben los algoritmos para estimar la dimensión de capacidad. El valor mínimo de $r$ corresponde a la mínima distancia entre los puntos presentes en los datos disponibles.

Si se generaliza el promedio de la ecuación (4.42),

$$
C_{q}(r)=\sqrt[q-1]{\left\langle\left[\mu\left(\mathcal{B}_{r}(\mathbf{x})\right)\right]^{q-1}\right\rangle}
$$

donde $C_{q}(r) \sim r^{D_{q}}, \mathrm{y}$

$$
D_{q}=\lim _{r \rightarrow 0} \frac{\log C_{q}(r)}{\log r} .
$$

Para $q=2$ se obtiene un promedio aritmético, la dimensión de correlación; para $q=1$ el promedio posibilita la estimación de la dimensión de información (media geométrica),

$$
C_{1}(r)=\lim _{q \rightarrow 1} C_{q}(r)=e^{\left\langle\log \mu\left(\mathcal{B}_{r}(\mathbf{x})\right)\right\rangle},
$$

A partir de un número finito $N$ de vectores de estado reconstruidos, $C_{q}(r)$ puede aproximarse como

$$
C_{q}(N, r)=\left\{\frac{1}{N} \sum_{i=1}^{N}\left[\frac{1}{N-1} \sum_{\substack{j=1 \\ i \neq j}}^{N} \Theta\left(r-\left\|\mathbf{x}_{i}-\mathbf{x}_{j}\right\|\right)\right]^{q-1}\right\}^{1 /(q-1)} .
$$

Para $q=2$ la ecuación (4.49) se reduce a la ecuación (4.45). La integral de correlación generalizada $C_{q}(N, r)$ engendra problemas computacionales para $q \leqslant 1$. 
Una variante a la integral de correlación generalizada comporta evaluar el número de tuplas de $q$ elementos, ${ }^{10}$ en las que las distancias entre pares de puntos, en el seno de cada tupla, no superen el umbral $r$ [Grassberger, 1983], esto es,

$$
C_{q}(N, r)=\frac{1}{N^{q}} \#\left\{\left(\mathbf{x}_{i_{1}}, \mathbf{x}_{i_{2}}, \ldots, \mathbf{x}_{i_{q}}\right):\left\|\mathbf{x}_{i}-\mathbf{x}_{j}\right\| \leqslant r, \forall i, j \in\left\{i_{1}, i_{2}, \ldots, i_{q}\right\}\right\} .
$$

Dado que $C_{q}(r) \sim r^{(q-1) D_{q}}$, las dimensiones generalizadas, conforme a las tuplas de $q$ elementos, obedecen a

$$
D_{q}=\lim _{r \rightarrow 0} \lim _{N \rightarrow \infty} \frac{1}{q-1} \frac{\log C_{q}(N, r)}{\log r} .
$$

\section{Algoritmos de masa fija}

En los algoritmos de masa fija se considera la variación de los tamaños de las bolas a fin de que todas ellas contengan la misma masa. En este caso se computa $\left\langle r_{k}\right\rangle$, que denota la distancia media al $k$-ésimo punto vecino más cercano. Si $d\left(\mathbf{x}_{i}, k\right)$ identifica la distancia que separa a $\mathbf{x}_{i}$ de su $k$-ésimo punto vecino más cercano,

$$
\left\langle r_{k}\right\rangle=\frac{1}{N} \sum_{i=1}^{N} d\left(\mathbf{x}_{i}, k\right) .
$$

El escalamiento $\left\langle r_{k}\right\rangle \sim k^{1 / d}$ permite estimar la dimensión $d$. A veces se evalúan los momentos de la distancia media [Badii and Politi, 1985, Grassberger, 1985, van de Water and Schram, 1988], manteniéndose $k$ a un valor fijo; de esta forma, la dimensión deriva del escalamiento del momento medio de $r_{k}$ en función del número de vectores de estado reconstruidos $N$, esto es,

$$
\left\langle r_{k}^{\gamma}\right\rangle \sim\left(\frac{k}{N}\right)^{\gamma / d(\gamma)}
$$

La función de dimensión $d(\gamma)$ se relaciona con las dimensiones generalizadas por medio de

$$
\begin{aligned}
\gamma & =(q-1) D_{q} \\
d(\gamma) & =D_{q} .
\end{aligned}
$$

\subsubsection{Entropía}

El estudio de sistemas complejos, en los que presumiblemente interaccionan muchos constituyentes, como ocurre en un sistema fisiológico, reclama el uso de métodos estadísticos; es lo que se llama mecánica estadística o termodinámica. ${ }^{11}$ La termodinámica estudia la relación que existe entre la energía desordenada y la energía

\footnotetext{
${ }^{10} \mathrm{El}$ número de tuplas de $q$ elementos crece rápidamente con $N$.

${ }^{11}$ La mecánica estadística pretende derivar las leyes de la termodinámica, a partir de la mecánica clásica, por medio de la estadística.
} 
ordenada. En la jerga física o química la energía totalmente desordenada recibe la denominación de calor. La primera ley de la termodinámica afirma que la energía total, ordenada más desordenada, no cambia con el tiempo. La segunda ley de la termodinámica sostiene que la evolución espontánea de todo sistema aislado nunca puede conducir a un decrecimiento de su entropía (desorden). La entropía siempre aumenta a medida que el sistema evoluciona. Si el sistema finalmente alcanza un estado de equilibrio, y ya no evoluciona, su entropía se mantiene constante, si bien esto último solo se manifiesta en contextos ideales. Dado que la entropía es una propiedad del estado actual del sistema y nunca disminuye con el tiempo, la evolución del sistema es irreversible, a diferencia de lo que ocurre en la mecánica, en que todo sistema es reversible. Esta paradoja, a veces expresada como la paradoja de la flecha del tiempo, queda zanjada con la entrada en escena del caos.

El caos está íntimamente ligado a la segunda ley de la termodinámica. Sin la presencia del caos esta quedaría anulada. El físico ruso Nikolay Sergeyevich Krylov (1917-1947) fue el primero en comprender esta relación [Krylov and Migdal, 1979]. La termodinámica se ocupa de sistemas muy grandes, con muchos constituyentes. De lo contrario no podría establecerse una distinción entre energía ordenada y calor. Por tanto, el espacio de estados presenta un número enorme de dimensiones. Si se conociera con exactitud el estado del sistema, este podría representarse como un punto en el espacio de estados. Como es obvio, es muy difícil determinarlo con precisión; únicamente se tiene un conocimiento parcial del estado actual, representado por una distribución de probabilidad del elemento de volumen que encierra el estado, que se caracteriza por una cantidad, la entropía. La entropía comprende una medida de la ausencia de conocimiento sobre el estado actual del sistema, pues dentro del elemento de volumen el estado verdadero podría ser cualquiera de los millones de puntos que lo conforman; la presencia de millones de posibilidades es lo que provoca un gran desconcierto, es la medida del desorden.

Los estados evolucionan con el tiempo, al igual que su distribución de probabilidad asociada, pero el elemento de volumen, aun modificando su morfología con el paso del tiempo, no cambia su volumen, de acuerdo con el teorema de Liouville; luego la entropía permanece constante con el tiempo, lo que contradice la segunda ley de la termodinámica. El caos resuelve esta discordancia. El elemento de volumen no modifica su volumen, pero su forma, con el tiempo y conforme a las particularidades del caos, se estira en unas direcciones y se contrae en otras, además de plegarse sobre sí misma. Cada punto del volumen sigue su propia trayectoria y trayectorias que inicialmente estaban muy próximas rápidamente divergen entre sí. La morfología que adquiere el elemento de volumen cada vez es más compleja, la estructura se hace más fina; se está construyendo un auténtico fractal. Cada vez se hace más difícil seguir los cambios que experimenta el verdadero estado, o sea, medir el volumen de la estructura. En definitiva el volumen inicial, compacto, se ha convertido en un ovillo de lana, con miles de agujeros (fractal). Si se reemplazara esta maraña de trayectorias por un volumen, sin agujeros, que las encerrara, el volumen final sería mayor que el inicial, y, por tanto, se habría incrementado la entropía, satisfaciéndose la segunda ley de la termodinámica. En la práctica, a nivel computacional, es la resolución numérica (coarse-graining) finita la que determina la mínima escala a la que puede evaluarse la distribución, perdiéndose los detalles más allá de la misma (smoothing). Esta 
privación constituye la ausencia de conocimiento y, por consiguiente, incrementa el volumen efectivo de la distribución, o, lo que es lo mismo, la entropía [Baranger, 2001].

Como ha sido expresado, el concepto de entropía se vincula profundamente al estudio de la mecánica estadística y de la termodinámica. La entropía comprende una cantidad termodinámica que describe el desorden presente en un sistema. En términos generales la entropía caracteriza la cantidad de información contenida en una distribución probabilística, de ahí su estrecha relación con la teoría de la información, que, desde los años 40, con las brillantes aportaciones de Claude E. Shannon, Alfréd Rényi y Andréi Kolmogórov, no ha dejado de dar fructíferos resultados en diferentes campos científicos [Desurvire, 2009].

La teoría de la información confiere una especial óptica al análisis de sucesiones temporales. Un sistema dinámico actúa como una fuente de información, una sucesión de números o símbolos, la sucesión temporal, interpretados como un mensaje transmitido. Si estos símbolos conforman una distribución de probabilidad, y las transiciones entre los símbolos están bien definidas en modo probabilístico, se suscitan cuestiones interesantes, como cuánto puede aprenderse del estado en que se encuentra el sistema cuando se efectúa una medición del sistema, o de cuánta información sobre futuras observaciones o mediciones se dispone atendiendo únicamente al pasado conocido [Farmer, 1982]. En este sentido la teoría de la información proporciona herramientas para, cuantitativamente, responder a las cuestiones planteadas, si bien su implementación logarítmica, en el ámbito de las sucesiones temporales, así como los detalles relacionados con la modelización de sistemas dinámicos, motivaron el desarrollo de dos disciplinas: la teoría de la información algorítmica [Chaitin, 2004] y la teoría de la información estadística [Kullback, 2012]. Por ejemplo, si el estado en que se encuentra un sistema dinámico se corresponde con un punto fijo estable, una simple medición u observación es suficiente para conocer el futuro del sistema; en el caso de un sistema periódico la observación de un simple período es suficiente para conocer la completa evolución futura del sistema. Si el sistema es aleatorio resulta imposible predecir el valor de la próxima medición, aun disponiéndose de infinitas observaciones pasadas. En este último escenario el valor medio de las pasadas observaciones se convierte en el mejor predictor del futuro inmediato.

\subsubsection{Entropías de una distribución estática}

Las entropías de Rényi de orden $q$ caracterizan la cantidad de información necesaria para especificar el valor de un observable [Rényi, 1961], con cierta precisión, cuando se entiende que la densidad de probabilidad de que un observable presente un valor $\mathbf{x}$ asciende a $\mathrm{d} \mu / \mathrm{d} \mathbf{x}$. Sea $\mathcal{P}_{\varepsilon}$ una cubierta del subespacio en que habita $\mathbf{x}$, compuesta por elementos autosimilares disjuntos $\mathcal{P}_{i}$ de diámetro $\leqslant \varepsilon$, esto es, la cubierta $\mathcal{P}_{\varepsilon}$ conforma una partición del subespacio en que habita $\mathbf{x}$. Si $p_{i}=\int_{\mathcal{P}_{i}} \mathrm{~d} \mu(\mathbf{x})$ comprende la fracción de la medida natural contenida en el $i$-ésimo elemento de la partición, entonces

$$
H_{q}\left(\mathcal{P}_{\varepsilon}\right)=\frac{1}{1-q} \ln \sum_{i} p_{i}^{q}
$$


define la entropía de Rényi de orden $q$ para una partición $\mathcal{P}_{\varepsilon}$. Para el caso $q=1$, la ecuación (4.55), evaluada de acuerdo con la regla de l'Hôpital, obedece a

$$
H_{1}\left(\mathcal{P}_{\varepsilon}\right)=-\sum_{i} p_{i} \ln p_{i}
$$

donde $H_{1}\left(\mathcal{P}_{\varepsilon}\right)$ coincide con la entropía de Shannon [Shannon and Weaver, 1998]. A diferencia de otras entropías de Rényi, la entropía $H_{1}\left(\mathcal{P}_{\varepsilon}\right)$ es aditiva, esto es, la entropía conjunta de varios procesos equivale a la suma de las entropías de los procesos independientes.

A diferencia de las dimensiones generalizadas, definidas en [Rényi, 1970], en las que los elementos de la cubierta no son disjuntos, en las entropías de Rényi de orden $q$ los elementos son disjuntos. En cualquier caso las dimensiones son los exponentes de escalamiento de las entropías de Rényi, evaluadas para elementos de igual tamaño de la partición que cubre el subespacio en cuestión, en función de $\varepsilon$, cuando $\varepsilon \rightarrow 0$.

\subsubsection{Entropía de Kolmogórov-Sinái}

La definición dada en la ecuación (4.55) adquiere mayor relevancia cuando se aplica a probabilidades de transición (evolución del sistema). Sea $\mathcal{P}_{\varepsilon}$ una partición del rango dinámico de un observable escalar, caso más típico de una sucesión temporal. Por tanto, los elementos de la partición conforman intervalos $I_{j}$ del eje real. La probabilidad conjunta $p_{i_{1}, i_{2}, \ldots, i_{m}}$, esto es, la probabilidad de que en un instante cualquiera $k$ el valor del observable esté contenido en el intervalo $I_{i_{1}}$, un tiempo $k+1$ el valor del observable esté contenido en el intervalo $I_{i_{2}}$, y así sucesivamente. Se define la entropía de bloque, para bloques de tamaño $m$, como

$$
H_{q}\left(m, \mathcal{P}_{\varepsilon}\right)=\frac{1}{1-q} \ln \sum_{i_{1}, i_{2}, \ldots, i_{m}} p_{i_{1}, i_{2}, \ldots, i_{m}}^{q} .
$$

Las entropías de orden $q$ obedecen a

$$
\begin{aligned}
& h_{q}=\sup _{\mathcal{P}} \lim _{m \rightarrow \infty} \frac{1}{m} H_{q}\left(m, \mathcal{P}_{\varepsilon}\right), \text { o equivalentemente } \\
& h_{q}=\sup _{\mathcal{P}} \lim _{m \rightarrow \infty} h_{q}\left(m, \mathcal{P}_{\varepsilon}\right)
\end{aligned}
$$

donde

$$
\begin{aligned}
h_{q}\left(m, \mathcal{P}_{\varepsilon}\right) & :=H_{q}\left(m+1, \mathcal{P}_{\varepsilon}\right)-H_{q}\left(m, \mathcal{P}_{\varepsilon}\right), \\
h_{q}\left(0, \mathcal{P}_{\varepsilon}\right) & :=H_{q}\left(0, \mathcal{P}_{\varepsilon}\right)
\end{aligned}
$$

En la ecuación (4.58) el supremo sup indica que ha de adquirirse el valor máximo sobre todas las posibles particiones $\stackrel{\mathcal{P}}{\mathcal{P}}$, lo que implica que $\varepsilon \rightarrow 0$. Si bien resulta factible estimar las entropías de Rényi de orden $q$ con probabilidades conjuntas, en 
sentido estricto es $h_{1}$ lo que se denomina entropía de Kolmogórov-Sinái o entropía KS [Kolmogórov, 1958, Sinái, 1959]. ${ }^{12}$

En el límite $q \rightarrow 0$ se obtiene la entropía topológica $h_{0}$; de la misma manera que $D_{0}$ contabiliza el número de elementos de una partición no vacíos, la entropía topológica detalla el número de órbitas periódicas inestables embebidas en el atractor.

(a)

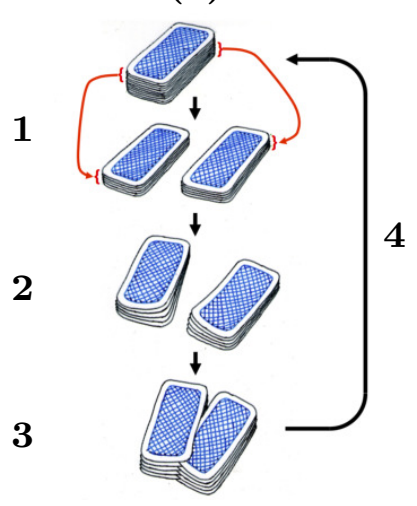

(b)

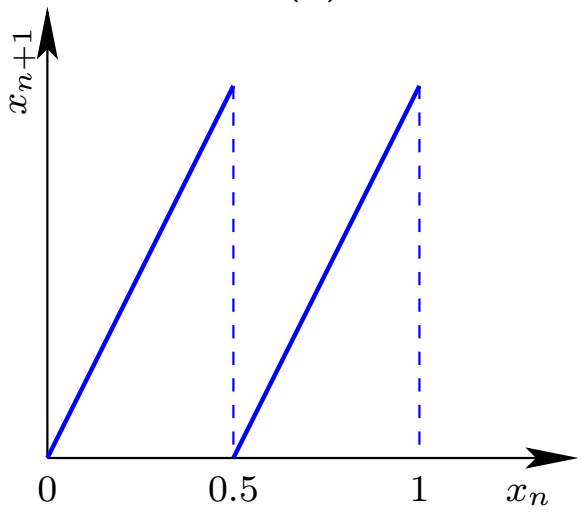

Figura 4.13: Mapa de desplazamiento de Bernoulli. En (a) se particulariza el procedimiento de estiramiento y plegado; en $\mathbf{1}$ se divide la baraja en dos montones exactamente iguales (el mapa es dos a uno); en $\mathbf{2}$ se curvan las puntas de las dos mitades hacia arriba hasta conseguir una altura que sea el doble que la altura original de cada montón (estiramiento); en $\mathbf{3}$ se entremezclan los dos montones arbitrariamente (plegado), del modo en que se baraja a la americana. Una vez que se dispone de un único montón, según $\mathbf{4}$, se repite el procedimiento tantas veces (iteraciones) como sean necesarias. En (b) se representa la característica onda de sierra de un mapa de desplazamiento de Bernoulli.

El mapa de desplazamiento de Bernoulli (Bernoulli shift), $x_{n+1} \equiv 2 x_{n}$ mód 1 , con $x_{n} \in[0,1)$, constituye un ejemplo paradigmático de lo que significa la entropía $\mathrm{KS}$, como ilustra la figura 4.13, en la que además se establece un símil entre la formulación entrópica del mapa de Bernoulli y la forma de proceder con una simple baraja de naipes [Dittrich, 2019]. Cuando se introduce un desplazamiento a un número real en el intervalo unidad $[0,1)$, su representación binaria se desplaza un bit a la izquierda, descartándose el bit más significativo (MSB). El bit menos significativo (LSB), con escasa información inicialmente, transcurridos unos pasos de tiempo, adopta un peso importante, mientras que el bit más significativo, que inicialmente proporcionaba mucha información, se ha perdido. Este flujo de información de las pequeñas a las grandes escalas es característico de los sistemas caóticos, y personifica otro aspecto de la dependencia sensible a las condiciones iniciales. Una representación gráfica del mapa de desplazamiento de Bernoulli semeja una onda de sierra (sawtooth wave). Debido al estiramiento por un factor de dos, el intervalo unidad se mapea a sí mismo dos veces, por lo que el mapa es dos a uno. Para que el atractor sea un subconjunto invariante, las tasas de estiramiento y plegado, en media, han de sumar cero; en este

\footnotetext{
${ }^{12}$ Andréi Kolmogórov y Yákov G. Sinái fueron los primeros en considerar las correlaciones temporales en el ámbito de la teoría de la información.
} 
sentido la entropía, a grandes rasgos, se refiere al logaritmo del número medio de pliegues. De hecho, la entropía topológica $h_{0}$ asume idéntico valor. En el caso de probabilidades de transición no uniformes se cumple que

$$
H_{q}(m) \geqslant H_{q^{\prime}}(m), \quad \text { si } q^{\prime}>q .
$$

Cuando se conoce el estado actual de un sistema con precisión finita, su representación binaria limitada, con un número finito de bits, imposibilita disponer de toda la información necesaria para especificar íntegramente el estado presente. Dado que la entropía, en el contexto del mapa de desplazamiento de Bernoulli, suministra la tasa a la que los bits son desplazados a la izquierda, es factible computar el tiempo $t_{p}$ requerido para que todos los bits que representan el estado actual hayan desaparecido en virtud del desplazamiento a izquierdas. Este tiempo se extrae de

$$
\varepsilon_{0} e^{h t_{p}}=1,
$$

donde $\varepsilon_{0}$ denota la resolución de las mediciones, cuyas magnitudes se escalan al intervalo unidad. Por tanto, en media, después de un tiempo

$$
t_{p}=-\frac{\ln \varepsilon_{0}}{h},
$$

toda capacidad predictiva se pierde completamente. Si se pretende extender linealmente el tiempo de predicción, debe incrementarse de forma exponencial la precisión de las mediciones. En general el tiempo de predicción experimenta fluctuaciones repentinas, fundamentalmente en aquellos episodios en que las amplitudes soportan cambios muy bruscos, introduciéndose incertidumbres puntuales que no modifican significativamente el tiempo promedio de predicción.

\subsubsection{Evolución temporal de la entropía}

La entropía KS puede extenderse a un tipo de entropía que depende de la escala $\varepsilon$, llamada entropía $\varepsilon$ por unidad de tiempo o entropía dinámica de grano grueso [Gaspard and Wang, 1993]. En la ecuación (4.57) la partición del espacio de estados $\mathcal{P}_{\varepsilon}$ depende explícitamente de $\varepsilon$, que representa el diámetro del elemento más grande de la partición. Por tanto, la entropía de bloque $H_{q}\left(m, \mathcal{P}_{\varepsilon}\right)$ contiene una longitud de escala, que desvela información adicional del sistema, a diferencia de la entropía KS, que se computa, conforme a la ecuación (4.58), en el límite $\varepsilon \rightarrow 0$.

La dificultad técnica de esta estrategia radica en el hecho de que no existe una partición única cuando se fija el valor de $\varepsilon$. Este contratiempo, derivado de la carencia de unicidad, se resuelve por medio de

$$
h_{q}(\varepsilon)=\inf _{\mathcal{P}_{\varepsilon}} \lim _{m \rightarrow \infty} H_{q}\left(m+1, \mathcal{P}_{\varepsilon}\right)-H_{q}\left(m, \mathcal{P}_{\varepsilon}\right),
$$

donde el ínfimo se adquiere sobre todas las particiones de diámetro $\varepsilon$, una vez fijado el valor de $\varepsilon$. Típicamente, en el límite $\varepsilon \rightarrow 0, h_{q}(\varepsilon) \rightarrow h_{q}$.

En el contexto de las sucesiones temporales un mejor cómputo de las entropías obliga a reemplazar la partición por una cubierta, dado que esta queda definida unívocamente para todo subconjunto de datos, y, por consiguiente, evita la estimación 
de cualquier ínfimo. Además, suele estimarse $h_{2}$ en vez de $h_{1}$, debido a su mejor comportamiento en presencia de sucesiones temporales de longitud finita. En cualquier caso el límite $m \rightarrow \infty$ no puede calcularse a partir de los datos, si bien el comportamiento de $H_{q}\left(m, \mathcal{P}_{\varepsilon}\right)$, para valores grandes y finitos de $m$, puede extrapolarse a $m \rightarrow \infty$.

Cabe señalar que relajar la condición $\varepsilon \rightarrow 0$ destruye la invariancia de $h_{q}(\varepsilon)$ bajo transformaciones de coordenadas infinitamente continuas. Por tanto, el valor de $h_{q}(\varepsilon)$ no es relevante; es más bien su dependencia de $\varepsilon$ y del tamaño del bloque $m$ lo que suministra una información valiosa.

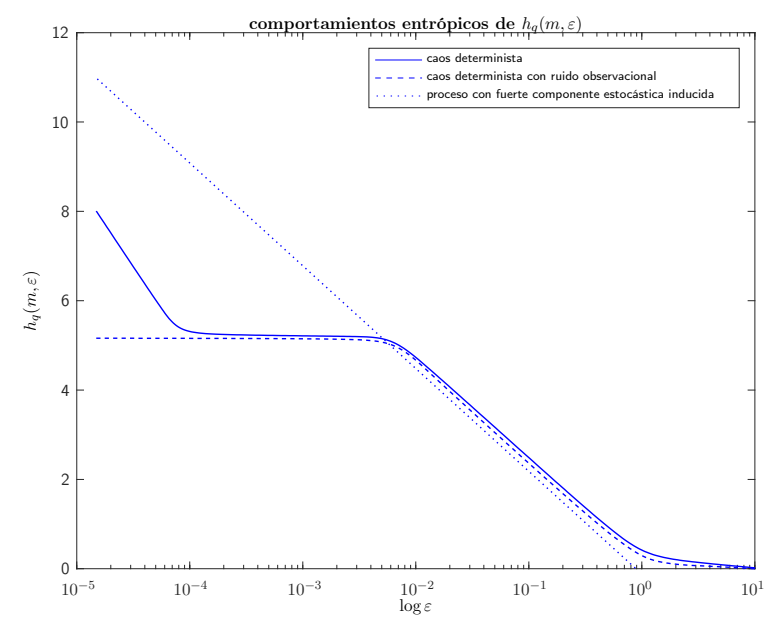

Figura 4.14: Comportamiento de $h_{q}(m, \varepsilon)$, con $q>0$, para tres diferentes procesos ilustrativos.

El comportamiento general de $h_{q}(m, \varepsilon)$, para diferentes escenarios, se ilustra en la figura 4.14. En el caso de valores grandes de $\varepsilon, h_{q}(m, \varepsilon)=0$. En caso de sistemas dinámicos deterministas, con un tamaño de bloque $m$ mayor que la dimensión del atractor, $h_{q}(m, \varepsilon) \rightarrow h_{q}(m)=$ constante, para valores pequeños de $\varepsilon$. Dependiendo del tipo de sistema y del tamaño del bloque $m, h_{q}(m)$ se convierte en una estimación aproximada de la entropía KS del sistema. Para un proceso estocástico, en el que las densidades de probabilidad son infinitamente continuas, o para un sistema determinista en el que el tamaño del bloque $m$ es menor que la dimensión del atractor, $h_{q}(m, \varepsilon)=-\ln \varepsilon+h_{q}^{(c)}(m)$, a pequeñas escalas $\varepsilon$. El factor $h_{q}^{(c)}(m)$ es una constante, denominada entropía condicional continua, y viene determinada por las entropías continuas de las correspondientes entropías de los bloques de longitud $m$, esto es,

$$
h_{q}^{(c)}(m)=H_{q}^{(c)}(m+1)-H_{q}^{(c)}(m)
$$

donde

$$
H_{q}^{(c)}(m)=\frac{1}{1-q} \ln \int\left(\rho^{(m)}(\mathbf{s})\right)^{q} \mathrm{~d} \mathbf{s},
$$

en que $\rho^{(m)}(\mathbf{s})$ identifica la densidad de probabilidad del proceso en un espacio de reconstrucción $m$-dimensional, y ds un elemento de volumen infinitesimal en el espacio 
de reconstrucción considerado. ${ }^{13}$

En un sistema determinista con ruido de medición u observacional la entropía dinámica diverge a pequeñas escalas. No obstante, en longitudes de escala intermedias $h_{q}(m, \varepsilon) \approx h_{q}$. Si se pretende caracterizar un sistema dinámico determinista en términos de su entropía, ha de reconocerse un aplanamiento de la entropía en longitudes de escala intermedias. La escasez de datos o la intensa amplitud del ruido de medición pueden desdibujar el aplanamiento de la función de entropía, a grandes escalas, complicándose la capacidad de distinción entre un proceso estocástico y un sistema determinista de alta dimensión y entropía elevada, como revela la figura 4.14.

La tasa de convergencia de la ecuación (4.63), cuando $m \rightarrow \infty$, refleja, en cierto sentido, la memoria del sistema, esto es, en qué medida las correlaciones lineales y no lineales del sistema se mantienen con el tiempo. En un sistema determinista la entropía tiende asintóticamente a un valor finito, es decir, $\operatorname{lím}_{\varepsilon \rightarrow 0} h_{q}(m, \varepsilon)=h_{q}(m)$, y $\lim _{m \rightarrow \infty} h_{q}(m)=h_{q}$, o sea, si no se consideran las correlaciones temporales la información del sistema se ve reducida. ${ }^{14}$

En definitiva la entropía dinámica se aplica de forma indistinta a sistemas estocásticos y a sistemas deterministas. Para bloques de tamaño $m$ suficientemente grande el comportamiento de la entropía, en función de $\varepsilon$, difiere significativamente en ambos tipos de sistemas. Si el valor de $\varepsilon$ es muy pequeño la dependencia de $m$ arroja algo de luz sobre la memoria del sistema, pudiendo llevar un análisis entrópico a discernir entre ruido y caos [Cencini et al., 2000]. En la práctica toda señal determinista contendrá varias componentes de ruido débiles, por lo que, junto con la insuficiencia de datos y la imposibilidad de alcanzar escalas muy pequeñas de $\varepsilon$, se menoscabaría la capacidad discriminante del análisis entrópico, si bien podrían extraerse propiedades interesantes del sistema bajo estudio.

\subsubsection{Entropías a partir de una sucesión temporal}

La entropía de una sucesión temporal proporciona información muy valiosa, pues la inversa de su valor numérico revela la escala temporal de predictibilidad del sistema. Además, la entropía de una sucesión temporal facilita información topológica acerca del proceso de plegado. Dado que tanto las entropías como los exponentes de Lyapunov se expresan en $1 / \mathrm{s}$, las magnitudes numéricas recabadas a partir de una sucesión temporal se indican en términos de $(\Delta t)^{-1}$, siendo $\Delta t$ el período de muestreo, si bien, a fin de poder comparar entropías de diferentes sistemas, es oportuno convertir las entropías a unidades de 1/s. En caso de extraer la entropía de un flujo a partir de una sección de Poincaré, resulta conveniente dividir la entropía obtenida por el intervalo temporal medio entre dos intersecciones consecutivas en la superficie de Poincaré, con objeto de contrastar el valor adquirido con la entropía del flujo de datos originario. En general los diferentes algoritmos que computan las entropías a

\footnotetext{
${ }^{13}$ En un proceso no correlado $\rho^{(m)}(\mathbf{s})=\prod_{k=1}^{m} \rho\left(s_{k}\right)$, de forma que las entropías continuas ascienden a $H_{q}^{(c)}(m)=m H_{q}^{(c)}(1)$ y $h_{q}^{(c)}(m)=H^{(c)}(1), \forall m$. Si los datos configuran una cadena de Markov de orden $l$, entonces $h_{q}^{(c)}(m)=h_{q}^{(c)}(l), \forall m \geqslant l$.

${ }^{14}$ Peter Grassberger sugiere $C_{\mathrm{emc}}=\sum_{m=1}^{\infty} h_{q}(m)-h_{q}$ como una medida de la complejidad efectiva del sistema (effective measure complexity) [Grassberger, 1986], si bien en sucesiones temporales de longitud finita esta medida no admite una estimación fiable.
} 
partir de una sucesión temporal operan con diferentes valores de $\tau \geqslant 1$, siendo $\tau$ el retardo temporal entre las diferentes componentes del vector de estado de un espacio de reconstrucción. En este sentido debiera dividirse la entropía extraída por el valor de $\tau$.

A diferencia de las dimensiones y de los exponentes de Lyapunov, no es fácil extraer los valores numéricos de las entropías de una sucesión temporal, debido fundamentalmente a que se requieren más datos para su cómputo, aparte de que no puede adquirirse el supremo de la ecuación (4.58) sobre todas las posibles particiones (no puede operarse con valores arbitrariamente pequeños de $\varepsilon$ ), ni sobre el límite $m \rightarrow \infty$ (estadísticos en un espacio de altas dimensiones).

Una implementación directa de la definición de la entropía KS se sirve del conteo de cajas (box-counting). Si los datos se escalan al intervalo unidad $[0,1)$, se cubre el espacio de fases por $1 / \varepsilon$ subintervalos de tamaño $\varepsilon$, determinándose las probabilidades conjuntas $p_{i_{1}, i_{2}, \ldots, i_{m}}$ por simplemente contar el número de segmentos $n_{i_{1}, i_{2}, \ldots, i_{m}}$ de la sucesión temporal contenidos en la secuencia de subintervalos $I_{i_{1}}, I_{i_{1}}, \ldots, I_{i_{m}}$. Este procedimiento equivale a evaluar un histograma $m$-dimensional. Aun con una asignación de memoria eficiente, en la que solo se reserva memoria para los subintervalos ocupados, ${ }^{15}$ el tamaño finito de los subintervalos introduce efectos de borde y un exiguo escalamiento. Por tanto, resulta beneficioso solapar los subintervalos, al igual que en la estimación de la dimensión, y distribuir, no uniformemente, los subintervalos de una manera más eficiente, a fin de alcanzar una mejor estimación de la medida natural (importance sampling).

En el caso de $q>1$, con subintervalos de tamaño fijo, a los que se asigna una probabilidad $p$, para dimensiones de reconstrucción $m$ en orden creciente, se obtiene

$$
C_{q}(m, \varepsilon) \propto \varepsilon^{(q-1) D_{q}} \varepsilon^{(1-q) H_{q}(m)},
$$

atendiendo al límite $\varepsilon \rightarrow 0$, dado que a priori se desconoce la partición generadora que conduce al supremo de $C_{q}(m, \varepsilon)$. En la práctica, debido a la longitud finita de la sucesión temporal y al ruido presente en los datos, el límite $\varepsilon \rightarrow 0$ es inalcanzable. En su lugar ha de reconocerse un rango de escalas en el que los resultados son independientes de $\varepsilon$. Esta independencia de la escala se manifiesta en forma de aplanamiento en la representación gráfica de los exponentes locales $D_{q}(m, \varepsilon)$. En caso contrario procede abandonar este análisis.

En el rango de escalas en que se aprecia un aplanamiento el factor $\varepsilon^{D_{q}}$ de la ecuación (4.66) es aproximadamente constante, posibilitándose la determinación del exponente $h_{q}$ por medio de la representación gráfica de

$$
h_{q}(m, \varepsilon)=H_{q}(m+1, \varepsilon)-H_{q}(m, \varepsilon)=\ln \frac{C_{q}(m, \varepsilon)}{C_{q}(m+1, \varepsilon)}
$$

vs. $m$, para un rango de escalas $\varepsilon$, y vs. $\varepsilon$, para diferentes valores de $m$.

Para valores de $m$ suficientemente grandes la ecuación (4.67) debiera converger a un valor constante $h_{q}$. Cómo de grande ha de ser $m$ está condicionado por las correlaciones no lineales del sistema. En procesos markovianos de orden $m, h_{q}\left(m^{\prime}\right)=h_{q}$,

\footnotetext{
${ }^{15}$ De hecho, las necesidades de memoria se reducen drásticamente; en general se ocupan $\varepsilon^{-D_{0}} e^{m h}$ subintervalos aproximadamente, de los $\varepsilon^{-m}$ totales.
} 
para $m^{\prime}>m$. La mayoría de los sistemas dinámicos no admiten su mapeo a procesos markovianos con memoria finita, lo que obliga a escoger valores de $m$ tan grandes como sea posible, a fin de establecer una fiel estimación de $h_{q}$. La dificultad estadística estriba en conseguir suficientes vecinos, dado que, de acuerdo con la ecuación (4.66), se pierden $e^{-h}$ vecinos cuando se pasa de $m$ a $m+1$.

$\mathrm{Al}$ igual que sucede con el cómputo de la dimensión, en el que la dimensión de correlación $D_{2}$ confiere una estimación muy robusta, la entropía $h_{2}$ se convierte en la opción más interesante a nivel computacional, dado que, para $q=2$, la suma de correlación se reduce a una media aritmética del número de vecinos, suministrando un resultado con sentido incluso cuando casi todos los puntos de referencia carecen de vecinos. Por consiguiente, el cómputo de $h_{2}$ posibilita operar con valores muy grandes de $m$.

El cálculo de la entropía KS, $h_{1}$, a partir de la integral de correlación generalizada, implícitamente exhibida en la ecuación (4.67), adolece de la eventual posibilidad de enfrentarse a vecindades vacías. En este sentido se recomienda hacer uso de una aproximación de masa fija, al igual que suele administrarse en el cómputo de $D_{1}$ a partir de los datos de una sucesión temporal [Badii and Politi, 1985, Grassberger, 1985]. De este modo, si se introduce la dependencia de $m$,

$$
\left\langle\varepsilon_{p}(m)^{(1-q) D_{q}}\right\rangle \propto\left(p e^{H_{q}(m)}\right)^{1-q}, \quad p \rightarrow 0 .
$$

En el límite $q \rightarrow 1$,

$$
\begin{aligned}
h_{1}(m, p) & =H_{1}(m+1, p)-H_{1}(m, p) \\
& =D_{1}\left(\left\langle\ln \varepsilon_{p}(m)\right\rangle-\left\langle\ln \varepsilon_{p}(m+1)\right\rangle\right) .
\end{aligned}
$$

Si $h_{1}$, conforme a la ecuación (4.69), se aproxima, en el régimen de escalamiento, a una magnitud finita para valores cada vez mayores de $m$, el límite asintótico constituye una estimación de la entropía KS, en adelante $h_{\mathrm{KS}}$.

\subsubsection{Relaciones entre los exponentes de Lyapunov, las dimensiones y las entropías}

Los exponentes de Lyapunov, las dimensiones y las entropías configuran diferentes perspectivas para describir las propiedades de la medida invariante de un sistema dinámico. Estas cantidades caracterizan distintos aspectos de la dinámica subyacente, de ahí que sea connatural establecer ciertas relaciones entre ellas.

\section{Identidad de Pesin}

Los exponentes de Lyapunov positivos determinan la divergencia exponencial de trayectorias muy próximas. Esta divergencia responde a la pérdida de información acerca de los posibles estados futuros del sistema. Los errores de medición introducen incertidumbre en la especificación del estado actual del sistema; una forma de simbolizar este error de medición consiste en rodear el valor observado o medido por una bola cuyo diámetro representa la amplitud del ruido. La evolución temporal 
deforma esta bola, transformándola en un elipsoide, cuyos semiejes son estirados y contraídos por factores $e^{\lambda_{i} t}$. Aunque el volumen total del elipsoide se reduce, debido a la disipación predominante, la incertidumbre sobre su posición futura crece con las longitudes de aquellos semiejes que se expanden. Si hubiere varios exponentes $\lambda_{i}$ positivos, cada una de las direcciones inestables asociadas contribuye al efecto de expansión. La identidad de Pesin asienta una primera relación entre la entropía de Kolmogórov-Sinái, o entropía KS, y los exponentes de Lyapunov positivos [Pesin, 1977], esto es,

$$
h_{\mathrm{KS}} \leqslant \sum_{i: \lambda_{i}>0} \lambda_{i}
$$

El sumatorio de los exponentes de Lyapunov positivos constituye una cota superior de la entropía KS. La identidad de Pesin es válida siempre que la medida natural sea continua a lo largo de las direcciones inestables [Ruelle, 1978]. En general la identidad de Pesin se cumple en el contexto de las medidas naturales de atractores, mientras que en subconjuntos inestables, como aquellos que repelen órbitas cercanas, la entropía es estrictamente menor que la suma de los exponentes de Lyapunov positivos. La identidad de Pesin admite una generalización cuando en su formulación se introduce el concepto de fractalidad [Ledrappier and Young, 1985], es decir,

$$
h_{\mathrm{KS}}=\sum_{i: \lambda_{i}>0} D_{i} \lambda_{i}=-\sum_{j: \lambda_{j}<0} D_{j} \lambda_{j}
$$

donde las dimensiones $D_{i}, D_{j}$ comprenden las dimensiones parciales, que definen las dimensiones de información de los subconjuntos formados por la intersección del atractor con las direcciones inestables, respecto a $\lambda_{i}$, y estables, referidas a $\lambda_{j}{ }^{16}$

Habitualmente, la identidad de Pesin se convierte en el procedimiento más eficaz para recabar una estimación veraz de la entropía KS de una sucesión temporal, dado que su cómputo directo se ve limitado por la insuficiencia de datos. No obstante, con objeto de atribuir cierta consistencia a los resultados obtenidos, se aconseja, al menos, calcular la entropía $h_{2}$. La entropía $h_{2}$ ha de ser una cota inferior de la entropía KS, y, por consiguiente, inferior a la suma de los exponentes de Lyapunov positivos. De la misma manera que en el caso de las dimensiones generalizadas $D_{q}$, una disparidad en los valores de $h_{q}$ puede interpretarse, no sin cierta reserva, debido tanto a errores sistemáticos como a errores estadísticos, como un signo de la presencia de multifractalidad en los datos.

\section{Conjetura de Kaplan-Yorke}

De carácter menos intuitivo que la relación entre la entropía y los exponentes de Lyapunov, la dimensión de información también se vincula a los exponentes de Lyapunov. Todo atractor es invariante bajo la evolución de su dinámica; esto significa que, cuando el atractor se considera un subvolumen $D_{1}$-dimensional, aquel ni se estira ni se contrae con el tiempo. Además, atrae puntos de su vecindad, dotándolo

\footnotetext{
${ }^{16}$ La segunda igualdad de la ecuación (4.71) solo se satisface en sistemas invertibles.
} 
de estabilidad ante pequeñas perturbaciones. Una vez conocido el espectro de exponentes de Lyapunov, puede inferirse el valor de $D_{1}$, de forma que un volumen típico, con esta dimensión, satisfaga las propiedades pertinentes.

Si un subconjunto finito de dimensión 1 experimenta únicamente un estiramiento a una tasa exponencial, marcada por el valor del mayor exponente de Lyapunov, esto es, se asume que $\lambda_{1}>0$, este subconjunto no es invariante. Si un área, de dimensión 2, experimenta además el influjo de un segundo exponente de Lyapunov negativo, con $\lambda_{2}<-\lambda_{1}$, aquel se estira en una dirección por un factor $e^{\lambda_{1} t}$, y se contrae en la otra dirección por un factor $e^{\lambda_{2} t}$, pero dado que $\left|\lambda_{2}\right|>\lambda_{1}$, el área se contrae en su conjunto por un factor $e^{-\left(\left|\lambda_{2}\right|-\lambda_{1}\right) t}$. En definitiva, un objeto fractal que ocupa las direcciones de contracción como si de un subconjunto de Cantor se tratara, presenta una dimensión parcial $D_{1}-1<1$. Para que este objeto sea invariante bajo la evolución temporal, $\lambda_{1}+\left(D_{1}-1\right) \lambda_{2}=0$, o, de otro modo, $D_{1}=1+\lambda_{1} /\left|\lambda_{2}\right|$. Estas observaciones sugieren que la parte entera de la dimensión del atractor corresponde al número de exponentes, en orden descendente, que pueden sumarse, de forma que la suma permanezca positiva. La parte fraccionaria se obtiene mediante una simple interpolación lineal.

En consecuencia, la dimensión de Kaplan-Yorke [Kaplan and Yorke, 1979], también bautizada como dimensión de Lyapunov $d_{L}$ (ver tabla 4.2), obedece a

$$
D_{\mathrm{KY}} \equiv d_{L}=k+\frac{\sum_{i=1}^{k} \lambda_{i}}{\left|\lambda_{k+1}\right|}
$$

donde $\sum_{i=1}^{k} \lambda_{i} \geqslant 0$, y $\sum_{i=1}^{k+1} \lambda_{i}<0$. La conjetura de Kaplan-Yorke establece que la dimensión de Lyapunov $D_{\mathrm{KY}}$ coincide con la dimensión de información $D_{1}$. Aunque esta conjetura ha sido corroborada únicamente en mapas bidimensionales [Ledrappier and Young, 1985], es razonable admitirla como una primera aproximación en cualquier sistema dinámico.

\subsection{Técnicas de medida de la complejidad}

En el ámbito del conocimiento científico aplicado a los sistemas dinámicos, de los que se dispone normalmente de uno o varios observables, adquiridos experimentalmente, una apreciación preliminar determinante, antes de proceder con las herramientas apropiadas de análisis de los datos, deriva de la capacidad para poder caracterizar la complejidad de un sistema dinámico, esto es, identificar la dinámica interna del proceso físico bajo estudio, de entre un amplio abanico de posibilidades, incluyendo los casos más sutiles, como discernir entre una dinámica caótica de dimensión baja y una evolución completamente estocástica, o procesos más extremos, como discriminar rotundamente entre una dinámica periódica y una dinámica caótica de baja dimensión. En este sentido sobresalen dos técnicas, una es el test $0-1$, principalmente por su fácil implementación con independencia de la información disponible del sistema físico subyacente, y el algoritmo de análisis de complejidad de Lempel-Ziv, especialmente útil en aplicaciones biomédicas como una medida para estimar la complejidad de señales en tiempo discreto. 


\subsubsection{Test $0-1$}

El test 0-1 permite distinguir una dinámica regular, periódica o cuasiperiódica, de una dinámica caótica, operando directamente sobre la sucesión temporal, sin recurrir a un preprocesamiento previo de los datos, como sucede con la reconstrucción del espacio de estados. Tanto los sistemas dinámicos continuos como los sistemas dinámicos discretos admiten el test 0-1. Típicamente, para computar los exponentes de Lyapunov de datos discretos, se requería la reconstrucción del espacio de estados. El test 0-1, desarrollado en [Gottwald and Melbourne, 2004, Gottwald and Melbourne, 2005, Gottwald and Melbourne, 2009a], se ejecuta directamente sobre la sucesión temporal, aun contaminada con ruido, ${ }^{17}$ sin preprocesamiento previo de los datos. El test $0-1$, con independencia de la dimensión presente en el sistema dinámico subyacente, consume pocos recursos computacionales.

\subsubsection{Descripción del test}

El test toma como entrada una sucesión temporal de datos escalares $s(n),{ }^{18}$ con $n=1,2, \ldots, N$, y genera, a partir de $s(n)$, un sistema bidimensional, a saber,

$$
\begin{aligned}
& p(n+1)=p(n)+s(n) \cos c n, \\
& q(n+1)=q(n)+s(n) \sin c n,
\end{aligned}
$$

donde $c \in(0,2 \pi)$ se fija a un valor constante. Se define el desplazamiento cuadrático medio (promedio temporal) $M(n)$ como

$$
M(n)=\lim _{N \rightarrow \infty} \frac{1}{N} \sum_{j=1}^{N-n}\left([p(j+n)-p(j)]^{2}+[q(j+n)-q(j)]^{2}\right), \quad n=1,2,3, \ldots
$$

Su tasa de crecimiento $K$ obedece a

$$
K=\lim _{n \rightarrow \infty} \frac{\log M(n)}{\log n} .
$$

Los valores de $K$ determinan el tipo de comportamiento de un sistema dinámico. Si $K=0$ el sistema dinámico se comporta de forma regular; si $K=1$ el sistema dinámico evoluciona de forma caótica. En el caso de sistemas dinámicos regulares las soluciones definidas por las ecuaciones del sistema (4.73) describen trayectorias acotadas; en el caso de sistemas dinámicos caóticos la evolución que experimentan las trayectorias propuestas en (4.73) se asemeja a la de un movimiento browniano 2-dimensional, esto es, su evolución es difusiva. El desplazamiento cuadrático medio $M(n)$ posibilita la identificación de la tasa de crecimiento de las trayectorias, ya sea

\footnotetext{
${ }^{17}$ Se considera habitualmente el ruido observacional (measurement noise), esto es, la diferencia entre el valor verdadero y el valor observado, debido a la imprecisión de la medición.

${ }^{18}$ La sucesión temporal $s(n)$ comprende un observable 1-dimensional del sistema dinámico subyacente. Un observable es todo aquello que puede medirse; luego un estado queda definido completamente por el valor de todos los observables.
} 
una tasa de crecimiento acotada (caso regular) o una tasa de crecimiento lineal (caso caótico). En cualquier caso el parámetro $K$ captura esta tasa de crecimiento.

\subsubsection{Descripción del algoritmo}

El test $0-1$ puede implementarse con pocas líneas de código. Sea una sucesión temporal $s(j)$, para $j=1,2, \ldots, N$, siendo $N$ el número de observaciones. Los pasos del algoritmo serían los siguientes:

1. Para $c \in(0, \pi)$, se resuelve el sistema (4.73), a fin de obtener

$$
\begin{aligned}
& p_{c}(n)=\sum_{j=1}^{n} s(j) \cos j c, \\
& q_{c}(n)=\sum_{j=1}^{n} s(j) \sin j c,
\end{aligned}
$$

con $n=1,2, \ldots, N$.

2. Con el fin de analizar el comportamiento difusivo, o no difusivo, de $p_{c}(n)$ y de $q_{c}(n)$, se computa $M_{c}(n)$, esto es,

$$
M_{c}(n)=\frac{1}{N} \sum_{j=1}^{N-n}\left(\left[p_{c}(j+n)-p_{c}(j)\right]^{2}+\left[q_{c}(j+n)-q_{c}(j)\right]^{2}\right), \quad n=1,2,3, \ldots
$$

Con objeto de asegurar que existe $M_{c}(n)$ cuando $N \rightarrow \infty$, se precisa que $n \ll N$. Por tanto, se calcula $M_{c}(n)$ únicamente para $n \leqslant N_{0}$, siendo $N_{0} \ll N$. En la práctica $N_{0}$ debería tomar un valor, a lo sumo, de $N / 10{ }^{19}$

En [Gottwald and Melbourne, 2009b] se propone una expresión similar a $M_{c}(n)$, en términos de comportamiento asintótico, con mejores propiedades de convergencia, esto es,

$$
D_{c}(n)=M_{c}(n)-V_{o s c}(c, n) .
$$

El término $V_{o s c}(c, n)$ obedece a

$$
V_{o s c}(c, n)=\langle s(n)\rangle^{2} \cdot \frac{1-\cos n c}{1-\cos c},
$$

siendo

$$
\langle s(n)\rangle=\lim _{N \rightarrow \infty} \frac{1}{N} \sum_{j=1}^{N} s(j) .
$$

\footnotetext{
${ }^{19}$ Una desventaja del test es que no se usan todos los datos disponibles en la sucesión temporal.
} 
3. La tasa de crecimiento asintótico $K_{c}$ de $M_{c}(n)$, o de su versión modificada $D_{c}(n)$, permite diferenciar un comportamiento regular de un comportamiento caótico. Para calcular $K_{c}$ se utilizan dos métodos: método de regresión y método de correlación.

- Método de regresión. Se emplea la regresión lineal sobre una representación logarítmica (log-log plot) de $M_{c}(n)$, o $D_{c}(n)$. En el caso de $M_{c}(n)$ la definición de $K_{c}$ atiende a la ecuación (4.75), a saber,

$$
K_{c}=\lim _{n \rightarrow \infty} \frac{\log M_{c}(n)}{\log n} .
$$

Numéricamente $K_{c}$ se determina ajustando una línea recta a la curva descrita por $\log M_{c}(n)$ frente a $\log n$, minimizando la desviación absoluta, en lugar del característico método de los mínimos cuadrados, dado que en este se atribuye un peso mayor a los valores atípicos (outliers). $D_{c}(n)$ exhibe menos varianza que $M_{c}(n)$, por lo que es natural aplicar el método de regresión a $D_{c}(n)$. No obstante, $D_{c}(n)$ podría adquirir valores negativos, debido a que se sustrae el término $V_{\text {osc }}(n)$ de $M_{c}(n)$, de ahí que se establece

$$
\tilde{D}_{c}(n)=D_{c}(n)+a \min _{1 \leqslant n \leqslant N_{0}}\left|D_{c}(n)\right|,
$$

donde $a>1$, por lo que ahora

$$
K_{c}=\lim _{n \rightarrow \infty} \frac{\log \tilde{D}_{c}(n)}{\log n} .
$$

De nuevo, $K_{c}$ puede determinarse numéricamente por regresión lineal, minimizándose la desviación absoluta, a partir de la gráfica de $\log \tilde{D}_{c}(n)$ frente a $\log n$.

- Método de correlación. Se construyen dos vectores, el vector $\xi=$ $\left(1,2, \ldots, N_{0}\right)$ y el vector $\Delta=\left(D_{c}(1), D_{c}(2), \ldots, D_{c}\left(N_{0}\right)\right)$, y se define el coeficiente de correlación entre ambos vectores, esto es,

$$
K_{c}=\operatorname{corr}(\xi, \Delta)=\frac{\operatorname{cov}(\xi, \Delta)}{\sqrt{\operatorname{var}(\xi) \operatorname{var}(\Delta)}} \in[-1,1] .
$$

El coeficiente $K_{c}$ evalúa el grado de correlación lineal entre $D_{c}(n)$ y un crecimiento lineal, caracterizado por $\xi$. El método de correlación ofrece mejores resultados que el método de regresión, según los análisis efectuados, para diferentes sistemas dinámicos, en [Gottwald and Melbourne, 2009b].

4. Se ejecutan los pasos 1 a 3 para varios valores de $c$; con 100 valores de $c$, seleccionados aleatoriamente, es suficiente. El resultado final de $K$ atiende a la 
mediana de los valores de $K_{c}$ computados, $K=$ mediana $\left(K_{c}\right) \cdot{ }^{20}$ Los valores de $c$ son seleccionados aleatoriamente en un intervalo $c \in(\pi / 5,4 \pi / 5)$, a fin de evitar resonancias. Las resonancias ocurren cuando en la dinámica del sistema se presenta una componente periódica, de frecuencia $\omega$, implicando un término, en la descomposición de Fourier de $s(n)$, proporcional a $\exp (-i \omega k)$. En este caso se manifiesta una resonancia en $c=\omega$, dando lugar a $p_{c}(n) \sim n$ y $q_{c}(n) \sim n$, y, por consiguiente, $M_{c}(n) \sim n^{2}$ y $D_{c}(n) \sim n^{2}$, lo que se traduce en $K_{c}=2$, para el método de regresión, y $K_{c}=1$ para el método de correlación. En el test se excluye $c=0$, dado que, con independencia del comportamiento dinámico subyacente, el sistema siempre entra en resonancia para $c=0$.

En cualquiera de los métodos utilizados un valor de $K \approx 0$ indica una dinámica regular, y un valor de $K \approx 1$ sugiere un comportamiento caótico. Normalmente, en todo método de detección de caos se reivindica una longitud de la sucesión temporal lo suficientemente larga como para que se manifieste el atractor en su conjunto, pues la veracidad de los tests depende del comportamiento asintótico del sistema subyacente, ya sea difusivo o no. Si la longitud de la sucesión temporal bajo estudio es corta, entra dentro de lo posible que el comportamiento asintótico, difusivo o no, carezca del efecto dominante que se persigue. En este caso, más que la convergencia de $K$ hacia 0 o 1 , se examina la tendencia de $K$ hacia 0 o 1 , respecto a la longitud de la sucesión temporal, con objeto de dictaminar la presencia o ausencia de caos.

La ventaja del test $0-1$ reside en su bajo coste computacional y en su facilidad de implementación, así como en su aplicabilidad general a cualquier sistema dinámico, siendo su naturaleza y su dimensión irrelevantes. Además, el test opera directamente sobre la sucesión temporal sin necesidad de recurrir a la reconstrucción del espacio de estados, y es especialmente hábil para detectar un comportamiento regular en presencia de ruido.

\subsubsection{Complejidad de Lempel-Ziv}

La complejidad de Lempel-Ziv [Lempel and Ziv, 1976] se fundamenta en la noción de complejidad de Andréi N. Kolmogórov [Kolmogorov, 1968], quien propuso como medida de complejidad la longitud del programa binario (núm. de bits) más corto capaz de generar la secuencia de datos considerada (complejidad algorítmica). Un algoritmo genérico para tal programa no ha sido aún suministrado. En este sentido Abraham Lempel y Jacob Ziv sugirieron un programa basado en dos operaciones, copiar e insertar. Además, ellos no calcularon la longitud del programa que genera una sucesión temporal de longitud $N$, sino más bien un número $c(n)$ que comporta una medida apropiada de la complejidad de Kolmogórov [Kaspar and Schuster, 1987]. De acuerdo con este planteamiento, la complejidad de Lempel-Ziv evalúa la complejidad de una secuencia finita de datos desde la perspectiva de una máquina de aprendizaje, a saber, dada una sucesión temporal $s_{j}$, para $j=1,2, \ldots, N$, siendo $N$ el número de observaciones, se examina la secuencia de $N$ dígitos $S=s_{1} s_{2} \cdots s_{N}$ de izquierda a derecha, añadiéndose una nueva palabra a la memoria cada vez que se descubre

\footnotetext{
${ }^{20}$ La mediana, en estadística, identifica el valor central de una muestra de observaciones o valores numéricos.
} 
una subcadena de dígitos consecutivos previamente no encontrada. El tamaño del vocabulario consignado y la tasa de ocurrencia de nuevas subcadenas o palabras (patrones) a medida que se escruta la secuencia $S$ sirven de base para establecer una métrica que revele la complejidad de $S$. En el contexto de las señales biológicas, la sucesión temporal $s_{j}$ es convertida a una sucesión binaria (alfabeto finito), conforme a unas reglas de transformación. Normalmente se suele recurrir a la mediana de los datos recabados como umbral entre lo que es un 0 y lo que es un 1 , esto es, si $T_{d}=\operatorname{mediana}\left(s_{j}\right)$, se construye una sucesión binaria $P=p_{1} p_{2} \cdots p_{N}$, conforme a

$$
p_{j}= \begin{cases}0, & \text { si } s_{j}<T_{d}, \\ 1, & \text { si } s_{j} \geqslant T_{d} .\end{cases}
$$

En cualquier caso el análisis de complejidad de Lempel-Ziv establece una partición de las mediciones u observaciones de acuerdo con una codificación simbólica de grano grueso [Aboy et al., 2006], de forma que a partir de la misma sea factible reconocer regularidades estructurales en la dinámica del sistema físico bajo estudio.

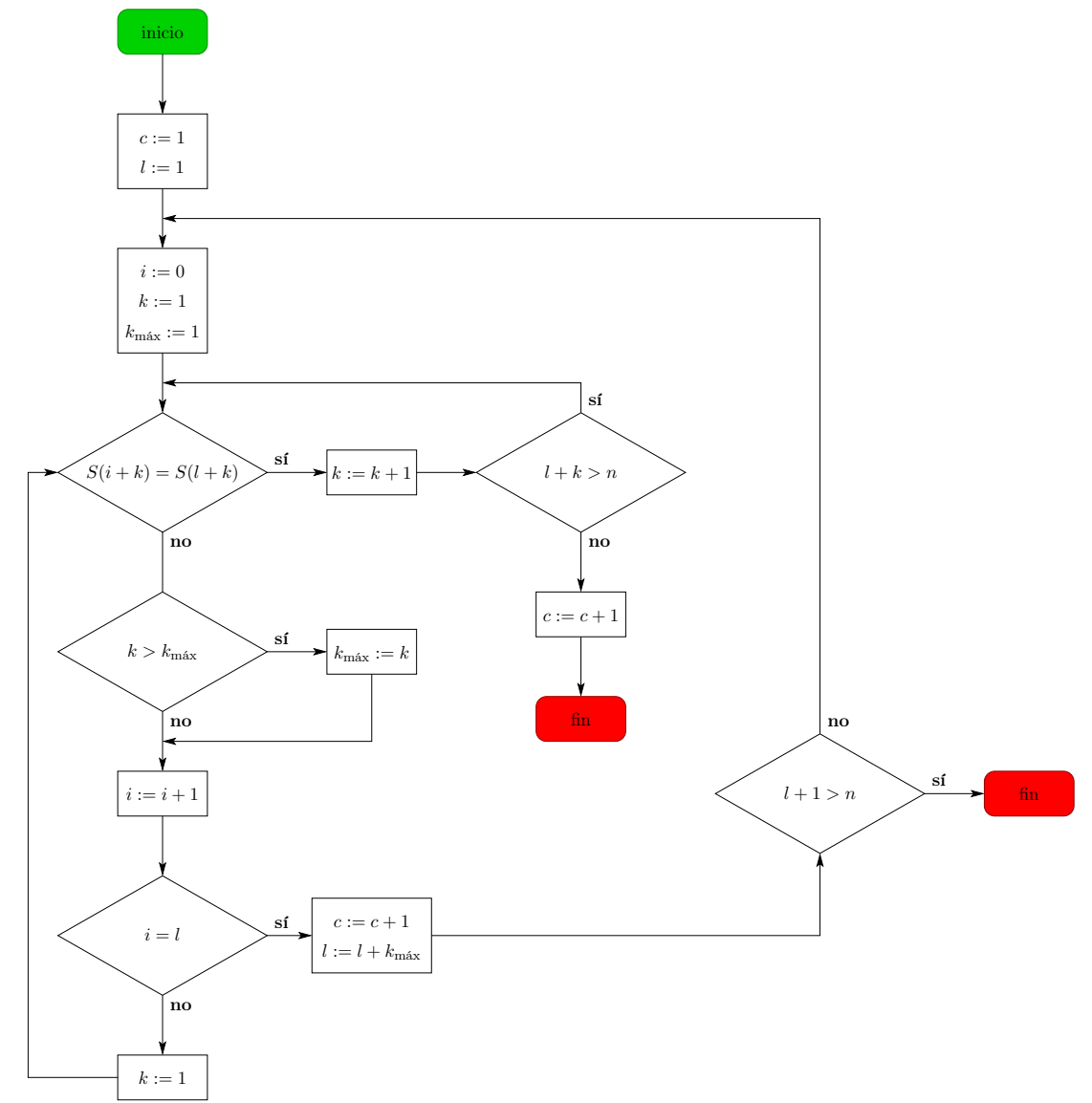

Figura 4.15: Diagrama de flujo del algoritmo que permite calcular $c(n)$ [Kaspar and Schuster, 1987]. 


\subsubsection{Descripción del algoritmo}

Dada una secuencia binaria $P$, de longitud $N$, ideada según algún criterio que posibilite, en función del sistema físico de interés, la detección de cambios estructurales en su dinámica [Aboy et al., 2006], se define un contador $c(n)$ que se incrementa en una unidad cada vez que se presenta una nueva subcadena de caracteres (ver diagrama de flujo en la figura 4.15).

1. Sean $S$ y $Q$ dos subcadenas de $P$ y $S Q$ el resultado de concatenar $S$ y $Q$. La subcadena $S Q \pi$ es el resultado de eliminar el último carácter de $S Q \cdot{ }^{21}$ Sea, además, $v(S Q \pi)$ el vocabulario que reúne todas las subcadenas diferentes de $S Q \pi$. En un principio $c(n)=1, S=p_{1}$ y $Q=p_{2}$; por tanto, $S Q \pi=p_{1}$.

2. En general $S=p_{1} p_{2} \cdots p_{r}, Q=p_{r+1}$ y $S Q \pi=p_{1} p_{2} \cdots p_{r}$. Si $Q$ pertenece a $v(S Q \pi)$, entonces $Q$ es una subcadena de $S Q \pi$ y no una nueva subcadena.

3. Si $Q$ pertenece a $v(S Q \pi)$ se anexa un nuevo carácter a $Q$, esto es, $Q$ pasa a ser $Q=p_{r+1} p_{r+2}$ y se examina de nuevo si este nuevo $Q$ pertenece a $v(S Q \pi)$.

4. Se repiten los pasos 2 y 3 hasta que $Q$ no pertenezca a $v(S Q \pi)$. De este modo, $Q=p_{r+1} p_{r+2} \cdots p_{r+i}$ no forma parte del vocabulario y, por consiguiente, no constituye una subcadena de $S Q \pi=p_{1} p_{2} \cdots p_{r+i-1}$. Así, $c(n)$ se incrementa en una unidad.

5. Acto seguido, una vez incorporada al vocabulario la nueva subcadena $Q$ se actualizan las variables de manera que ahora $S=p_{1} p_{2} \cdots p_{r+i}$ y $Q=p_{r+i+1}$, procediéndose nuevamente con los pasos 2, 3 y 4 .

El procedimiento anterior se repite hasta que $Q$ alcance al último carácter de $P$. Llegados a este punto el número de diferentes subcadenas presentes en $P$ queda registrado en $c(n)$, que representa la complejidad de la sucesión $P$.

A fin de obtener una medida de la complejidad normalizada e independiente de la longitud de $P$, el contador $c(n)$ ha de ser normalizado, esto es, si $N$ comprende la longitud de la sucesión $P$ y el número de símbolos del alfabeto $A$ asciende a $\alpha=|A|$, una cota superior de $c(n)$ obedece a

$$
c(n)<\frac{N}{\left(1-\varepsilon_{N}\right) \log _{\alpha}(N)},
$$

donde $\varepsilon_{N} \rightarrow 0$ cuando $N \rightarrow \infty .^{22}$ En este sentido

$$
\lim _{N \rightarrow \infty} c(n)=b(n)=\frac{N}{\log _{\alpha} N} .
$$

Para una secuencia binaria $\alpha=2$, y, por tanto,

$$
b(n)=\frac{N}{\log _{2} N} .
$$

\footnotetext{
${ }^{21} \mathrm{El}$ símbolo $\pi$ denota la operación de borrado del último carácter de la subcadena que precede al propio símbolo.

${ }^{22} \mathrm{El}$ factor $\varepsilon_{N}$ suele ser muy pequeño, puesto que $\varepsilon_{N}=2 \frac{1+\log (\alpha N)}{\log N}$ [Lempel and Ziv, 1976].
} 
De esta forma $c(n)$ puede normalizarse por medio de $b(n)$, es decir,

$$
C=\frac{c(n)}{b(n)},
$$

donde $C$ identifica la complejidad normalizada de Lempel-Ziv, que refleja la tasa de ocurrencia de patrones que van surgiendo en la secuencia binaria conforme progresa la dinámica de la sucesión temporal.

La complejidad de Lempel-Ziv permite caracterizar patrones espacio-temporales incluso en sistemas de muy alta dimensión, en los que el espectro de exponentes de Lyapunov, u otras medidas entrópicas, puede resultar impracticable desde un punto de vista numérico y computacional [Kaspar and Schuster, 1987]. Los patrones obtenidos no suministran información acerca de la dinámica del sistema en el espacio de estados, o, dicho con otras palabras, no revelan, como así abordan otras técnicas [Halsey et al., 1987], la estructura geométrica del posible atractor consubstancial al proceso físico. 



\section{Referencias}

[Abarbanel et al., 1990] Abarbanel, H. D. I., Brown, R., and Kadtke, J. B. (1990). Prediction in chaotic nonlinear systems: Methods for time series with broadband fourier spectra. Physical Review A, 41(4):1782-1807. (página 104).

[Abarbanel et al., 1991] Abarbanel, H. D. I., Brown, R., and Kennel, M. B. (1991). Lyapunov exponents in chaotic systems: their importance and their evaluation using observed data. International Journal of Modern Physics B, 5(9):1347-1375. (página 106).

[Abarbanel et al., 1992] Abarbanel, H. D. I., Brown, R., and Kennel, M. B. (1992). Local lyapunov exponents computed from observed data. Journal of Nonlinear Science, 2(3):343-365. (página 100).

[Abarbanel et al., 1993] Abarbanel, H. D. I., Brown, R., Sidorowich, J. J., and Tsimring, L. S. (1993). The analysis of observed chaotic data in physical systems. Reviews of Modern Physics, 65(4):1331-1392. (páginas 52, 57, 58, 103, 165).

[Aboy et al., 2006] Aboy, M., Hornero, R., Abasolo, D., and Alvarez, D. (2006). Interpretation of the lempel-ziv complexity measure in the context of biomedical signal analysis. IEEE Transactions on Biomedical Engineering, 53(11):2282-2288. (páginas 139, 140).

[Akay et al., 2000] Akay, M., in Medicine, I. E., and Society, B. (2000). Nonlinear Biomedical Signal Processing, Dynamic Analysis and Modeling. IEEE Press Series on Biomedical Engineering. Wiley. (página 98).

[Alligood et al., 2012] Alligood, K., Sauer, T., and Yorke, J. (2012). Chaos: An Introduction to Dynamical Systems. Textbooks in Mathematical Sciences. Springer Berlin Heidelberg. (página 97).

[Arnold, 1965] Arnold, V. I. (1965). Small denominators, i: mappings of the circumference onto itself. American Mathematical Society, 46:213-284. (página 87).

[Assouad, 1979] Assouad, P. (1979). Étude d'une dimension métrique liée à la possibilité de plongements dans $\mathbf{R}^{n}$. C. R. Acad. Sci. Paris Sér. A-B, 288(15):A731A734. (página 116).

[Badii and Politi, 1985] Badii, R. and Politi, A. (1985). Statistical description of chaotic attractors: The dimension function. Journal of Statistical Physics, 40(56):725-750. (páginas 123, 132). 
[Bak, 1996] Bak, P. (1996). How nature works: the science of self-organized criticality. Copernicus Series. Copernicus. (página 117).

[Balatoni and Rényi, 1976] Balatoni, J. and Rényi, A. (1976). On the notion of entropy. In English translation: Selected papers of Alfréd Rényi, volume 1, pages 558-584. Publ. Math. Inst. Hungarian Acad. Sci. (1956), Akademiat Kiado, Budapest. (página 116).

[Baranger, 2001] Baranger, M. (2001). Chaos, complexity, and entropy - a physics talk for non-physicists. Wesleyan University Physics Dept. Colloquium. (página $125)$.

[Bergé et al., 1987] Bergé, P., Pomeau, Y., and Vidal, C. (1987). Order within Chaos: towards a deterministic approach to turbulence. Wiley-VCH. (página 91).

[Brown et al., 1991] Brown, R., Bryant, P., and Abarbanel, H. D. I. (1991). Computing the lyapunov spectrum of a dynamical system from an observed time series. Physical Review A, 43(6):2787-2806. (página 107).

[Bryant et al., 1990] Bryant, P., Brown, R., and Abarbanel, H. D. I. (1990). Lyapunov exponents from observed time series. Physical Review Letters, 65(13):15231526. (página 107).

[Bryant, 1992] Bryant, P. H. (1992). Computation of lyapunov exponents from experimental data. In Proceedings of the 1st Experimental Chaos Conference, pages 11-23. World Scientific. (página 104).

[Buczkowski et al., 1998] Buczkowski, S., Kyriacos, S., Nekka, F., and Cartilier, L. (1998). The modified box-counting method: Analysis of some characteristic parameters. Pattern Recognition, 31(4):411-418. (página 116).

[Cencini et al., 2000] Cencini, M., Falcioni, M., Olbrich, E., Kantz, H., and Vulpiani, A. (2000). Chaos or noise: Difficulties of a distinction. Physical Review E, 62(1):427-437. (página 130).

[Chaitin, 2004] Chaitin, G. J. (2004). Algorithmic Information Theory. Cambridge Tracts in Theoretica. Cambridge University Press. (página 125).

[Collet and Eckmann, 2009] Collet, P. and Eckmann, J.-P. (2009). Iterated Maps on the Interval as Dynamical Systems. Birkhäuser Boston. (página 93).

[Crawford, 1991] Crawford, J. D. (1991). Introduction to bifurcation theory. Reviews of Modern Physics, 63(4):991-1037. (página 88).

[Crutchfield et al., 1986] Crutchfield, J. P., Farmer, J. D., Packard, N. H., and Shaw, R. S. (1986). Chaos. Scientific American, 254(12):46-57. (página 95).

[Cvitanović, 1989] Cvitanović, P. (1989). Universality in Chaos, 2nd edition. Taylor \& Francis. (página 95). 
[Dämmig and Mitschke, 1993] Dämmig, M. and Mitschke, F. (1993). Estimation of lyapunov exponents from time series: the stochastic case. Physics Letters A, 178(5-6):385-394. (página 111).

[Desurvire, 2009] Desurvire, E. (2009). Classical and Quantum Information Theory: An Introduction for the Telecom Scientist. Cambridge University Press. (página 125).

[Dittrich, 2019] Dittrich, T. (2019). Quantum chaos and quantum randomnessparadigms of entropy production on the smallest scales. Entropy. (página 127).

[Doyon et al., 1993] Doyon, B., Cessac, B., Quoy, M., and Samuelides, M. (1993). Control of the transition to chaos in neural networks with random connectivity. International Journal of Bifurcation and Chaos, 3(2):279-291. (página 92).

[Eckmann et al., 1986] Eckmann, J.-P., Kamphorst, S. O., Ruelle, D., and Ciliberto, S. (1986). Liapunov exponents from time series. Physical Review A, 34(6):49714979. (página 107).

[Eckmann and Ruelle, 1985] Eckmann, J.-P. and Ruelle, D. (1985). Ergodic theory of chaos and strange attractors. Reviews of Modern Physics, 57(3):617-656. (páginas $36,47,105,106,165,213)$.

[Eckmann and Ruelle, 1992] Eckmann, J.-P. and Ruelle, D. (1992). Fundamental limitations for estimating dimensions and lyapunov exponents in dynamical systems. Physica D: Nonlinear Phenomena, 56(2-3):185-187. (páginas 68, 105).

[Engelking, 1978] Engelking, R. (1978). Dimension theory. North-Holland Publishing Company. (página 112).

[Falconer, 2003] Falconer, K. (2003). Fractal Geometry: Mathematical Foundations and Applications. John Wiley \& Sons. (página 116, 116).

[Farmer, 1982] Farmer, J. D. (1982). Information dimension and the probabilistic structure of chaos. Zeitschrift für Naturforschung A, 37(11):1304-1326. (páginas $112,125)$.

[Farmer et al., 1983] Farmer, J. D., Ott, E., and Yorke, J. A. (1983). The dimension of chaotic attractors. Physica D: Nonlinear Phenomena, 7(1-3):153-180. (páginas $36,114)$.

[Feigenbaum, 1978] Feigenbaum, M. J. (1978). Quantitative universality for a class of nonlinear transformations. Journal of Statistical Physics, 19(1):25-52. (página 93, 93).

[Feigenbaum, 1980] Feigenbaum, M. J. (1980). Universal behavior in nonlinear systems. Los Alamos Science, 1:4-27. (página 92).

[Fell et al., 1993] Fell, J., Röschke, J., and Beckmann, P. (1993). Deterministic chaos and the first positive lyapunov exponent: a nonlinear analysis of the human electroencephalogram during sleep. Biological Cybernetics, 69(2):139-146. (página 111). 
[Fujisaka, 1983] Fujisaka, H. (1983). Statistical dynamics generated by fluctuations of local lyapunov exponents. Progress of Theoretical Physics, 70(5):1264-1275. (página 100).

[Gao and Zheng, 1993] Gao, J. and Zheng, Z. (1993). Local exponential divergence plot and optimal embedding of a chaotic time series. Physics Letters A, 181(2):153158. (páginas 108, 109, 110).

[Gaspard and Wang, 1993] Gaspard, P. and Wang, X.-J. (1993). Noise, chaos, and $(\varepsilon, \tau)$-entropy per unit time. Physics Reports, 235(6):291-343. (página 128).

[Glass and Kaplan, 1993] Glass, L. and Kaplan, D. (1993). Time series analysis of complex dynamics in physiology and medicine. Medical progress through technology, 19(3):115-28. (página 106).

[Glazier and Libchaber, 1988] Glazier, J. A. and Libchaber, A. (1988). Quasiperiodicity and dynamical systems: An experimentalist's view. IEEE Transactions on Circuits and Systems, 35(7):790-809. (página 87).

[Gottwald and Melbourne, 2004] Gottwald, G. A. and Melbourne, I. (2004). A new test for chaos in deterministic systems. Proceedings of the Royal Society of London. Series A: Mathematical, Physical and Engineering Sciences, 460(2042):603-611. (páginas 135, 220).

[Gottwald and Melbourne, 2005] Gottwald, G. A. and Melbourne, I. (2005). Testing for chaos in deterministic systems with noise. Physica D: Nonlinear Phenomena, 212(1-2):100-110. (páginas 135, 220).

[Gottwald and Melbourne, 2009a] Gottwald, G. A. and Melbourne, I. (2009a). On the implementation of the 0-1 test for chaos. SIAM Journal on Applied Dynamical Systems, 8(1):129-145. (páginas 135, 220).

[Gottwald and Melbourne, 2009b] Gottwald, G. A. and Melbourne, I. (2009b). On the validity of the 0-1 test for chaos. Nonlinearity, 22(6):1367-1382. (páginas 136, 137, 221).

[Grassberger, 1983] Grassberger, P. (1983). Generalized dimensions of strange attractors. Physics Letters A, 97(6):227-230. (página 123).

[Grassberger, 1985] Grassberger, P. (1985). Generalizations of the hausdorff dimension of fractal measures. Physics Letters A, 107(3):101-105. (páginas 65, 123, 132).

[Grassberger, 1986] Grassberger, P. (1986). Toward a quantitative theory of selfgenerated complexity. International Journal of Theoretical Physics, 25(9):907-938. (página 130).

[Grassberger, 1988] Grassberger, P. (1988). Finite sample corrections to entropy and dimension estimates. Physics Letters A, 128(6-7):369-373. (páginas 67, 122, 213). 
[Grassberger and Procaccia, 1983a] Grassberger, P. and Procaccia, I. (1983a). Characterization of strange attractors. Physical Review Letters, 50(5):346-349. (página 116).

[Grassberger and Procaccia, 1983b] Grassberger, P. and Procaccia, I. (1983b). Measuring the strangeness of strange attractors. Physica D: Nonlinear Phenomena, 9(1-2):189-208. (páginas 66, 116, 121, 213, 213).

[Grassberger and Procaccia, 1984] Grassberger, P. and Procaccia, I. (1984). Dimensions and entropies of strange attractors from a fluctuating dynamics approach. Physica D: Nonlinear Phenomena, 13(1-2):34-54. (página 100).

[Grebogi et al., 1982] Grebogi, C., Ott, E., and Yorke, J. A. (1982). Chaotic attractors in crisis. Physical Review Letters, 48(22):1507-1510. (página 91).

[Grebogi et al., 1983] Grebogi, C., Ott, E., and Yorke, J. A. (1983). Crises, sudden changes in chaotic attractors, and transient chaos. Physica D: Nonlinear Phenomena, 7(1-3):181-200. (páginas 91, 96).

[Greenside et al., 1982] Greenside, H. S., Wolf, A., Swift, J., and Pignataro, T. (1982). Impracticality of a box-counting algorithm for calculating the dimensionality of strange attractors. Physical Review A, 25(6):3453-3456. (página 120).

[Halsey et al., 1987] Halsey, T. C., Jensen, M. H., Kadanoff, L. P., Procaccia, I., and Shraiman, B. I. (1987). Fractal measures and their singularities: The characterization of strange sets. Nuclear Physics B - Proceedings Supplements, 2:501-511. (páginas 116, 141).

[Hentschel and Procaccia, 1983] Hentschel, H. G. E. and Procaccia, I. (1983). The infinite number of generalized dimensions of fractals and strange attractors. Physica D: Nonlinear Phenomena, 8(3):435-444. (página 115).

[Higuchi, 1988] Higuchi, T. (1988). Approach to an irregular time series on the basis of the fractal theory. Physica D: Nonlinear Phenomena, 31(2):277-283. (páginas $116,208)$.

[Hopf, 1948] Hopf, E. (1948). A mathematical example displaying features of turbulence. Communications on Pure and Applied Mathematics, 1(4):303-322. (página 95).

[Hurewicz and Wallman, 1941] Hurewicz, W. and Wallman, H. (1941). Dimension Theory. Princeton mathematical series. Princeton University Press. (páginas 112, 116).

[Jensen et al., 1984] Jensen, M. H., Bak, P., and Bohr, T. (1984). Transition to chaos by interaction of resonances in dissipative systems. i. circle maps. Physical Review A, 30(4):1960-1969. (página 87).

[Kadanoff, 1983] Kadanoff, L. P. (1983). Roads to chaos. Physics Today, 36(12):4653. (página 91). 
[Kantz, 1994] Kantz, H. (1994). A robust method to estimate the maximal lyapunov exponent of a time series. Physics Letters A, 185(1):77-87. (páginas 108, 109, 110, 170).

[Kantz and Schreiber, 2004] Kantz, H. and Schreiber, T. (2004). Nonlinear Time Series Analysis. Cambridge nonlinear science series. Cambridge University Press. (páginas 97, 98, 103).

[Kaplan and Yorke, 1979] Kaplan, J. L. and Yorke, J. A. (1979). Chaotic behavior of multidimensional difference equations. In Functional Differential Equations and Approximation of Fixed Points, pages 204-227. Springer Berlin Heidelberg. (páginas 116, 134).

[Kaspar and Schuster, 1987] Kaspar, F. and Schuster, H. G. (1987). Easily calculable measure for the complexity of spatiotemporal patterns. Physical Review A, 36(2):842-848. (páginas 138, 139, 141).

[Kinsner, 2005] Kinsner, W. (2005). A unified approach to fractal dimensions. In Fourth IEEE Conference on Cognitive Informatics, 2005. (ICCI 2005)., pages 5872. IEEE. (página 115).

[Kolmogórov, 1958] Kolmogórov, A. (1958). A new metric invariant of transient dynamical systems and automorphisms in lebesgue spaces. Dokl. Akad. Nauk SSSR, 119(5):861-864. (página 127).

[Kolmogorov, 1968] Kolmogorov, A. N. (1968). Three approaches to the quantitative definition of information*. International Journal of Computer Mathematics, 2(14):157-168. (páginas 138, 222).

[Krylov and Migdal, 1979] Krylov, N. S. and Migdal, J. S. (1979). Works on the Foundations of Statistical Physics. Princeton Series in Physics. Princeton University Press. (página 124).

[Kullback, 2012] Kullback, S. (2012). Information Theory and Statistics. Dover Books on Mathematics. Dover Publications. (página 125).

[Landau and Lifshitz, 1987] Landau, L. D. and Lifshitz, E. M. (1987). Fluid mechanics, 2nd edition. Pergamon Press. (página 95).

[Ledrappier and Young, 1985] Ledrappier, F. and Young, L.-S. (1985). The metric entropy of diffeomorphisms: Part i: Characterization of measures satisfying pesin's entropy formula. Annals of Mathematics, 122(3):509-539. (páginas 133, 134).

[Lempel and Ziv, 1976] Lempel, A. and Ziv, J. (1976). On the complexity of finite sequences. IEEE Transactions on Information Theory, 22(1):75-81. (páginas 138, $140,222)$.

[Liapounoff, 1948] Liapounoff, A. M. (1948). Problème général de la stabilité du mouvement. (AM-17), Volume 17. Princeton University Press. (página 99). 
[liu Yang et al., 2012] liu Yang, H., Radons, G., and Kantz, H. (2012). Covariant lyapunov vectors from reconstructed dynamics: The geometry behind true and spurious lyapunov exponents. Physical Review Letters, 109(24). (página 107).

[Mandelbrot, 1988] Mandelbrot, B. (1988). Random fluctuations and pattern growth. NATO ASI Series E157, page 279. cited By 1. (páginas 115, 116, 116).

[Mandelbrot, 1983] Mandelbrot, B. B. (1983). The Fractal Geometry of Nature. W. H. Freeman. (páginas 33, 71, 112).

[Mettin et al., 1993] Mettin, R., Parlitz, U., and Lauterborn, W. (1993). Bifurcation structure of the driven van der pol oscillator. International Journal of Bifurcation and Chaos, 3(6):1529-1555. (página 87).

[Moon, 1992] Moon, F. C. (1992). Chaotic and Fractal Dynamics: Introduction for Applied Scientists and Engineers. Wiley. (página 86).

[Munkres, 2000] Munkres, J. R. (2000). Topology, 2nd edition. Featured Titles for Topology Series. Prentice Hall, Incorporated. (página 103).

[Nese, 1989] Nese, J. M. (1989). Quantifying local predictability in phase space. Physica D: Nonlinear Phenomena, 35(1-2):237-250. (página 100).

[Nicolis et al., 1983] Nicolis, J. S., Meyer-Kress, G., and Haubs, G. (1983). Nonuniform chaotic dynamics with implications to information processing. Zeitschrift für Naturforschung A, 38(11):1157-1169. (página 101).

[Oseledets, 1968] Oseledets, V. I. (1968). A multiplicative ergodic theorem: Ljapunov characteristic numbers for dynamical systems. Transactions of the Moscow Mathematical Society, 19:197-231. (página 99).

[Parker et al., 1989] Parker, T. S., Chua, L., and O., L. (1989). Practical Numerical Algorithms for Chaotic Systems. Springer New York. (página 102).

[Parlitz, 1992] Parlitz, U. (1992). Identification of true ans spurious lyapunov exponents from time series. International Journal of Bifurcation and Chaos, 2(1):155165. (página 107).

[Percival, 1989] Percival, I. (1989). Chaos: a science for the real world. New Scientist, 124(1687):42-47. (página 99).

[Pesin, 1977] Pesin, Y. B. (1977). Characteristic lyapunov exponents and smooth ergodic theory. Russian Mathematical Surveys, 32(4):55-114. (páginas 133, 211).

[Poincaré, 1885] Poincaré, H. (1885). Sur l'équilibre d'une masse fluide animée d'un mouvement de rotation. Acta Mathematica, 7(0):259-380. (página 87).

[Rényi, 1961] Rényi, A. (1961). On measures of entropy and information. In Proceedings of the Fourth Berkeley Symposium on Mathematical Statistics and Probability, Volume 1: Contributions to the Theory of Statistics, pages 547-561, Berkeley, Calif. University of California Press. (páginas 116, 125). 
[Rényi, 1970] Rényi, A. (1970). Probability theory. North-Holland series in applied mathematics and mechanics. Elsevier. (páginas 116, 116, 126).

[Robinson, 1982] Robinson, A. L. (1982). Physicists try to find order in chaos. Science, 218(4572):554-556. (página 96).

[Romera García, 1997] Romera García, M. (1997). Técnica de los sistemas dinámicos discretos. Colección textos universitarios: Consejo Superior de Investigaciones Científicas. Consejo Superior de Investigaciones Científicas. CSIC Press. (página 87).

[Rosenstein et al., 1993] Rosenstein, M. T., Collins, J. J., and Luca, C. J. D. (1993). A practical method for calculating largest lyapunov exponents from small data sets. Physica D: Nonlinear Phenomena, 65(1-2):117-134. (páginas 108, 109, 110).

[Ruelle, 1978] Ruelle, D. (1978). Thermodynamic Formalism: The Mathematical Structures of Classical Equilibrium Statistical Mechanics. Encyclopedia of mathematics and its applications. Addison-Wesley Publishing Company, Advanced Book Program. (página 133).

[Ruelle and Takens, 1971] Ruelle, D. and Takens, F. (1971). On the nature of turbulence. Comm. Math. Phys., 20(3):167-192. (páginas 32, 95).

[Sano and Sawada, 1985] Sano, M. and Sawada, Y. (1985). Measurement of the lyapunov spectrum from a chaotic time series. Physical Review Letters, 55(10):10821085. (páginas 105, 106, 204).

[Sato et al., 1987] Sato, S., Sano, M., and Sawada, Y. (1987). Practical methods of measuring the generalized dimension and the largest lyapunov exponent in high dimensional chaotic systems. Progress of Theoretical Physics, 77(1):1-5. (páginas $108,109,110,111)$.

[Sauer et al., 1998] Sauer, T. D., Tempkin, J. A., and Yorke, J. A. (1998). Spurious lyapunov exponents in attractor reconstruction. Physical Review Letters, 81(20):4341-4344. (página 107).

[Schuster and Just, 2006] Schuster, H. G. and Just, W. (2006). Deterministic Chaos: An Introduction, 4th edition. John Wiley \& Sons. (páginas 96, 97).

[Schützenberger, 1954] Schützenberger, M.-P. (1954). Contributions aux applications statistiques de la théorie de l'information. PhD thesis, Institut de Statistique de l'Université de Paris. (página 116).

[Shannon and Weaver, 1998] Shannon, C. E. and Weaver, W. (1998). Mathematical Theory of Communication. University of Illinois Press. (página 126).

[Sinái, 1959] Sinái, Y. (1959). On the concept of entropy of a dynamical system. Dokl. Akad. Nauk SSSR, 124(4):768-771. (página 127).

[Smale, 1967] Smale, S. (1967). Differentiable dynamical systems. Bull. Amer. Math. Soc., 73(6):747-817. (página 88). 
[Sprott, 2003] Sprott, J. C. (2003). Chaos and Time-Series Analysis. Oxford University Press. (páginas 34, 86, 96).

[Stoop and Parisi, 1991] Stoop, R. and Parisi, J. (1991). Calculation of lyapunov exponents avoiding spurious elements. Physica D: Nonlinear Phenomena, 50(1):8994. (página 108).

[Strogatz, 1994] Strogatz, S. H. (1994). Nonlinear Dynamics And Chaos. Studies in nonlinearity. Perseus Books Publishing, LLC. (páginas 32, 34, 91).

[Swinney, 1983] Swinney, H. L. (1983). Observations of order and chaos in nonlinear systems. Physica D: Nonlinear Phenomena, 7(1-3):3-15. (páginas 91, 92).

[Theiler, 1986] Theiler, J. (1986). Spurious dimension from correlation algorithms applied to limited time-series data. Physical Review A, 34(3):2427-2432. (página 122).

[Theiler, 1990] Theiler, J. (1990). Estimating fractal dimension. Journal of the Optical Society of America A, 7(6):1055-1073. (páginas 109, 117).

[Theiler et al., 1991] Theiler, J., Galdrikian, B., Longtin, A., Eubank, S., and Farmer, J. D. (1991). Using surrogate data to detect nonlinearity in time series. In Nonlinear Modeling and Forecasting, pages 163-88. (página 106).

[Tricot, 1982] Tricot, C. (1982). Two definitions of fractional dimension. Mathematical Proceedings of the Cambridge Philosophical Society, 91(1):57-74. (página 116).

[Tsonis, 2012] Tsonis, A. (2012). Chaos: From Theory to Applications. Springer US. (página 98).

[Tsonis, 1989] Tsonis, A. A. (1989). Chaos and unpredictability of weather. Weather, 44(6):258-263. (página 95).

[Unakafova and Keller, 2013] Unakafova, V. and Keller, K. (2013). Efficiently measuring complexity on the basis of real-world data. Entropy, 15(10):4392-4415. (páginas 98, 217).

[van de Water and Schram, 1988] van de Water, W. and Schram, P. (1988). Generalized dimensions from near-neighbor information. Physical Review A, 37(8):31183125. (página 123).

[Wiggins, 1996] Wiggins, S. (1996). Introduction to Applied Nonlinear Dynamical Systems and Chaos (Texts in Applied Mathematics, Vol 2). Springer-Verlag. (página 88).

[Williams, 1997] Williams, G. P. (1997). Chaos Theory Tamed. Joseph Henry Press. (página 91).

[Wolf et al., 1985] Wolf, A., Swift, J. B., Swinney, H. L., and Vastano, J. A. (1985). Determining lyapunov exponents from a time series. Physica D: Nonlinear Phenomena, 16(3):285-317. (páginas 105, 111). 



\section{Capítulo 5}

\section{Señales biológicas}

\subsection{Introducción a las señales biológicas}

Durante mucho tiempo los diferentes campos del saber estuvieron muy compartimentados, sin apenas conexión entre ellos. Es más, ni siquiera físicos reconocidos, como Copernico o Galileo, fueron verdaderos físicos. En aquella época las diferentes disciplinas universitarias giraban en torno a la medicina, la jurisprudencia o la teología, lo que impedía delimitar más específicamente subáreas de marcado carácter experimental [Beuter et al., 2003]. En este sentido las primeras investigaciones fisiológicas, como hoy serían juzgadas, datan del siglo XVIII, cuando el italiano Luigi Galvani (1737-1798), inspirado por las historias novelescas que de Benjamin Franklin se difundían, intentó, sin éxito, explicar el fenómeno de la «electricidad animal», a partir de las contracciones musculares que detectó mientras diseccionaba ranas. Tuvo que ser finalmente Alessandro Volta (1745-1827), precursor de la actual batería, quien diera con la clave, como así quedó consignado en su tesis doctoral.

Siguiendo con el recorrido histórico, aparece la figura del físico alemán Herman von Helmholtz (1821-1894), quien, además de sus célebres tratados sobre física, afrontó estudios fisiológicos relacionados con la audición y la visión ocular. Posteriormente, el fisiólogo inglés Archibald V. Hill (1886-1977) contribuyó, con sus trabajos teóricos y ensayos prácticos en la Universidad de Cambridge, a una explicación mecánica y termodinámica de la contracción muscular. Unos años más tarde, en 1952 Bernard Katz y Paul Fatt, de la University College de Londres, publicaron un trabajo influyente sobre cómo se efectúa la transmisión sináptica. De lo que no fueron conscientes, en aquel entonces, era de que su estudio insinuaba cómo un proceso determinista generaba datos con comportamiento estocástico.

En 1888 el físico-químico alemán Walter H. Nernst (1864-1941) centró su atención en el transporte de cargas eléctricas en soluciones electrolíticas, si bien fue su compatriota Max K. Planck (1858-1947), padre, junto con el austríaco Erwin Schrödinger (1887-1961), de la mecánica cuántica, quien precisó la ecuación matemática que describía el flujo electrolítico. Estos estudios cayeron en el olvido hasta que en 1930 Kenneth C. Cole y David Goldman, de la Universidad de Columbia, se sirvieron de esta ecuación para explicar el transporte iónico a través de la membrana celular, con el que pudieron demostrar cómo aumentaba la conductancia de la membrana 
durante el potencial de acción. La segunda guerra mundial supuso un freno para las investigaciones, si bien, aun así, Kenneth C. Cole, contratado por el MIT, y sus colaboradores fueron capaces de construir un dispositivo electrónico que les permitió medir alteraciones de la membrana celular en presencia del potencial de acción. En ese tiempo el fisiólogo inglés Allan L. Hodgkin (1914-1998) estaba en EE. UU. y valoró la posibilidad de usar este aparato para examinar lo que sucedía en el axón del calamar gigante durante el potencial de acción, ardua empresa que comenzó, a su regreso a Inglaterra, en colaboración con el fisiólogo inglés Andrew F. Huxley (1917-2012), y que les llevó a recibir el premio Nobel de Fisiología y Medicina en 1963.

El modelo matemático de Hodgkin-Huxley, que describe la excitabilidad de la membrana del axioma del calamar gigante, está compuesto por una ecuación diferencial parcial no lineal acoplada a tres ecuaciones diferenciales ordinarias. En la década de los 60 el fisiólogo americano Richard D. FitzHugh (1922-2007), de la Universidad de Colorado, se aventuró, junto con la asistencia de Jin-Ichi Nagumo (1926-2000), físico-matemático de la Universidad de Tokio, a reducir matemáticamente el modelo de Hodgkin-Huxley. Finalmente, fue todo un logro, que atiende al nombre de modelo de FitzHugh-Nagumo, y que ha facilitado enormemente la comprensión de la complejidad que encierra el proceso de excitabilidad celular. Tanto es así que el físico alemán Erwin Neher (1944-) y el fisiólogo conciudadano Bert Sakmann (1942-) pudieron demostrar la existencia de los canales iónicos a nivel de membrana, lo que les convirtió en merecedores del premio Nobel de Fisiología y Medicina en 1991. Además, diversas variantes del modelo de Hodgkin-Huxley han arrojado resultados notables en el caso del tejido cardíaco, como los cosechados por los fisiólogos americanos J. Walter Woodbury y Wayne E. Crill.

Si todo lo anterior se refiere a la configuración eléctrica de una sola célula, otros investigadores enfocaron sus estudios al análisis del comportamiento colectivo de agrupaciones celulares. Así, los neurocientíficos americanos Warren S. McCulloch (1898-1969) y Walter H. Pitts (1923-1969) demostraron en 1923 cómo un conjunto de neuronas podía llevar a cabo operaciones lógicas. Este descubrimiento supuso el punto de partida de lo que hoy día conforman las redes neuronales. En 1946 el polifacético matemático americano Norbert Wiener (1894-1964), padre de la cibernética, y el cardiólogo mejicano Arturo Rosenblueth (1900-1970) examinaron a nivel matemático el comportamiento de células cardíacas excitables, entendidas estas como una colección compleja de sistemas de control.

En el estudio funcional del sistema nervioso resalta la figura del fisiólogo estadounidense Haldan K. Hartline (1903-1983), de la Universidad Johns Hopkins y director de tesis de Richard FitzHugh, quien, habiendo completado su formación académica en Alemania, tanto en matemáticas como en física, dedicó la mayor parte de su vida al estudio de la fisiología de la retina del cangrejo de herradura, por el que le concedieron el premio Nobel de Fisiología y Medicina en 1967.

A tenor de las escuetas líneas previas, resulta evidente el carácter multidisciplinario de las ciencias de la vida, en las que la mayoría de los progresos han venido de la mano de las matemáticas y de la física. De hecho, libros, como ¿Qué es la vida? [Schrödinger, 1984], del físico austríaco Erwin Schrödinger, y Apología de un matemático [Hardy et al., 1999], ensayo escrito por el matemático inglés Godfrey H. 
Hardy (1877-1947), han servido de inspiración a generaciones de científicos en los campos de la biología molecular y de la genética, respectivamente. En los últimos años muchos matemáticos están dirigiendo sus esfuerzos a resolver problemas del mundo de la biología, a diferencia de lo que sucedió en siglos precedentes, en los que todos los desarrollos atendían a cuestiones surgidas de la física. Lo que antes se concentraba en un mismo individuo, como es el caso de Richard FitzHugh, actualmente se extrapola a equipos multidisciplinarios, en los que la activa colaboración entre sus miembros, además de disponer de un buen repertorio de bases de datos de alta calidad, resultará clave en el éxito de sus investigaciones.

\subsubsection{Señales biológicas}

La vertiente ingenieril de la biomedicina comporta la medición, el procesamiento y el análisis de las señales, así como la visualización de los resultados obtenidos. Dado que las señales son variaciones de energía que transportan información, el análisis de señales biológicas tiene por objetivo transformarlas de forma que sea más fácil extraer la información contenida en ellas.

La formas de energía que suelen medirse habitualmente en el cuerpo humano se reducen a la energía eléctrica, la energía química, la energía mecánica y la energía térmica [Semmlow and Griffel, 2014]. La información que ha de transportarse en el interior del cuerpo humano se codifica principalmente como variaciones de las energías eléctrica, en términos de flujo de iones, ${ }^{1}$ o química, en términos de concentración de constituyentes químicos, sin desatender otras variaciones energéticas, como ilustra la tabla 5.1.

Tabla 5.1: Formas de energía biológicas y variables de medida.

\begin{tabular}{|lll|}
\hline \hline energía & \multicolumn{1}{c}{ variables } & \multicolumn{1}{c|}{ mediciones } \\
\hline química & concentración química & $\begin{array}{l}\text { concentraciones en sangre, } \mathrm{O}_{2}, \mathrm{CO}_{2}, \\
\mathrm{pH}, \text { hormonas, etc. } \\
\text { mecánica }\end{array}$ \\
& posición, torque, presión & $\begin{array}{l}\text { contracción muscular, presión car- } \\
\text { diovascular, sonidos cardíacos, etc. }\end{array}$ \\
eléctrica & $\begin{array}{l}\text { voltaje y corriente del flujo ió- } \\
\text { nico }\end{array}$ & EEG, ECG, EMG, PPG, etc. \\
térmica & temperatura & termografía clínica \\
\hline \hline
\end{tabular}

En cualquier caso las diferentes formas de energía biológicas deben convertirse a energía eléctrica, a fin de procesar la información contenida en un ordenador. En

\footnotetext{
${ }^{1}$ Los portadores de carga iónicos del cuerpo humano son, por antonomasia, el $\mathrm{Na}^{+}$, el $\mathrm{K}^{+}$y el $\mathrm{Cl}^{-}$.
} 
el contexto de las señales biológicas el dispositivo biotransductor, que convierte las diferentes formas de energía en energía eléctrica, es un elemento crítico en aquellos sistemas ideados para captar señales procedentes de sistemas fisiológicos.

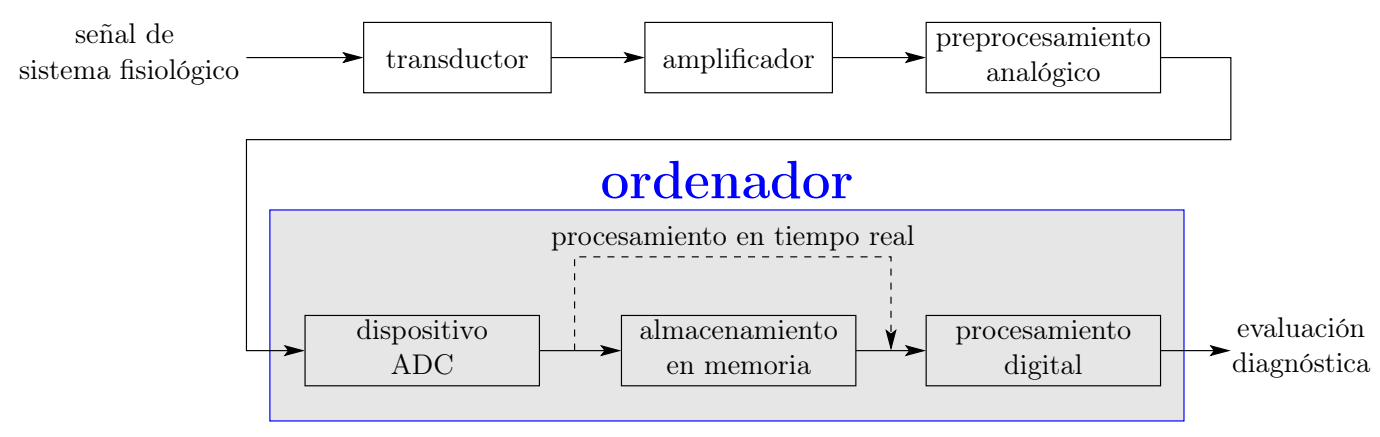

Figura 5.1: Esquema general de un sistema de adquisición de datos biomédicos.

Los sistemas de adquisición de datos fisiológicos, como refleja el esquema general de la figura 5.1, permiten captar señales biológicas y almacenarlas digitalmente en un ordenador para su posterior análisis con fines clínicos o de investigación. En primer lugar la información (fluctuación energética) contenida en los procesos fisiológicos bajo estudio ha de convertirse a una señal eléctrica, por medio del biotransductor. Dado que normalmente a la señal eléctrica se le acopla algún tipo de ruido, consecuencia de los mismos procesos de medición y de conversión, se requiere un prefiltrado de la misma, como un filtro paso bajo o un filtro paso banda, con objeto de eliminar el ruido aditivo fuera de banda, antes de proceder a la digitalización de la señal mediante un conversor analógico-digital (ADC). Una vez almacenada la señal en el ordenador, resulta factible aplicar técnicas digitales de procesamiento de señales, con diferentes grados de sofisticación en función del propósito perseguido, como evaluaciones diagnósticas o clasificaciones tumorales, si bien las tendencias actuales apuntan a la búsqueda de indicadores que, en tiempo real, determinen prematuramente, con un índice de acierto elevado, enfermedades que puedan poner en peligro la integridad física del individuo.

\subsection{Comportamientos dinámicos en fisiología}

Como ya se esquematizó en la tabla 4.1 se admite una categorización general en la clasificación de los sistemas dinámicos. Por un lado, figuran los sistemas aleatorios, y, por otro lado, los sistemas deterministas. Dejando a un lado la concepción determinista del universo, según la mecánica clásica de Newton, y defendida fervientemente por el matemático francés Pierre-Simon Laplace, la ingeniería suele considerar todos los sistemas como si así lo fueran, adoptando el ruido acoplado al sistema en calidad de proceso aleatorio. En un sistema determinista el curso futuro de las variables de estado es conocido, siempre que se disponga de un conocimiento preciso de sus valores, aparte de las leyes que pautan su evolución. En cambio en un sistema aleatorio es imposible predecir el curso futuro del sistema, sujeto completamente al azar, con independencia de los estados pasados, como sucede en la emisión radioactiva, un 
auténtico proceso aleatorio. No obstante, ningún sistema finito es verdaderamente aleatorio, como la generación por ordenador de números pseudoaleatorios, y con un número finito de datos aleatorios siempre es factible construir un modelo capaz de reproducir fielmente los datos adquiridos.

Un sistema lineal se caracteriza por dos propiedades: el escalamiento y la superposición. El escalamiento significa que si una señal de entrada $x(t)$ produce una señal de salida $y(t)$, al escalar la señal de entrada por un factor $a$, la señal de salida resulta escalada por idéntico factor, esto es,

$$
\text { si } x(t) \rightarrow y(t) \text {, entonces } a x(t) \rightarrow a y(t) .
$$

Atendiendo a la superposición, si una señal de entrada $x_{1}(t)$ produce una señal de salida $y_{1}(t)$, y una señal de entrada $x_{2}(t)$ produce una señal de salida $y_{2}(t)$, la suma de las señales de entrada produce una señal de salida que coincide con la suma de las señales de salida para sendas señales de entrada, o sea,

$$
\text { si } x_{1}(t) \rightarrow y_{1}(t) \wedge x_{2}(t) \rightarrow y_{2}(t) \text {, entonces } x_{1}(t)+x_{2}(t) \rightarrow y_{1}(t)+y_{2}(t) \text {. }
$$

Las propiedades de escalamiento y superposición simplifican los problemas sobremanera, propiciando un análisis muy sencillo de los sistemas, al menos desde una perspectiva teórica. Sin embargo, en el mundo real los sistemas no proceden de esa manera, y los sistemas fisiológicos son inherentemente no lineales. De todas formas, no es infrecuente que los sistemas físicos puedan operar en el régimen lineal para un determinado rango de valores de entrada, y respondan de forma no lineal, rebasados los niveles de saturación, fuera de ese rango de valores.

Una vez catalogados los diferentes tipos de sistemas más comunes, cabe subrayar que la mayoría de los sistemas fisiológicos no se enmarcan en un tipo particular, sino más bien abarcan una combinación de todos ellos, y lo que los hace especialmente interesantes es la variedad de comportamientos dinámicos que exhiben, más que su inspección taxonómica. En cualquier caso no está de más caracterizar su tipología, a fin de evaluar el dominio de aplicación de las técnicas de análisis.

Aunque el ruido está presente en todo proceso de medición de las variables de estado de un sistema físico, el balance entre la componente determinista de la evolución temporal de las variables de estado y la componente aleatoria acoplada no siempre se decanta a favor de la primera. No es fácil discernir si la aleatoriedad es consustancial a la propia dinámica del sistema o si, por el contrario, se asocia únicamente al proceso de medición o, inclusive, al ruido externo.

Un punto fijo comprende el escenario más simple en cuanto a comportamiento de un sistema determinista. Una vez alcanzado el estado de equilibrio, en el que las diferentes variables de estado retienen un valor concreto, el sistema permanece en ese estado indefinidamente, a menos que sea excitado o perturbado externamente, como sucede en el caso de un péndulo simple con fricción.

En un peldaño más arriba, en términos de complejidad, se sitúan los sistemas periódicos, en los que los sucesivos estados que puede atravesar el sistema se repiten cada cierto tiempo $T$, siendo $T$ el período de recurrencia de los estados. El prototipo más habitual al que se recurre es el movimiento armónico simple, característico de, 
por ejemplo, un péndulo no amortiguado que, en ausencia de fuerza externa, oscilaría eternamente a una frecuencia determinada. En el contexto de los sistemas no lineales los ciclos límite muestran una evolución periódica un tanto sutil; un ciclo límite describe una trayectoria periódica aislada, esto es, estados próximos a la trayectoria pueden verse atraídos por ella, en cuyo caso el ciclo límite es estable, o pueden ser repelidos por la trayectoria, lo que convierte al ciclo límite en inestable. En suma, existe alguna vecindad de la trayectoria periódica que no contiene otras órbitas periódicas. En un sistema lineal no pueden evidenciarse ciclos límite, dado que los estados próximos a una trayectoria periódica pertenecen a oscilaciones distintas y no son atraídos ni repelidos por ella, es decir, la trayectoria periódica no está aislada.

Una ligera, pero trascendente, modificación del concepto de periodicidad lo constituye la noción de cuasiperiodicidad. Un sistema cuasiperiódico integra procesos periódicos cuyos períodos se acoplan de forma irracional, esto es, la relación entre los diferentes períodos no puede expresarse como una razón de números enteros; con otras palabras, y sobre la base de dos señales, la cuasiperiodicidad es el resultado de emplear una portadora periódica, de período $T_{p}$, para modular en amplitud una señal moduladora, de período $T_{m}$, de forma que la relación entre el período de la señal moduladora (envolvente) y el período de la señal portadora es irracional (inconmensurable), $T_{p} / T_{m} \in \mathbb{I}$.

Por último, los sistemas deterministas que cubren una excepcional heterogeneidad dinámica, con multiplicidad de comportamientos que se extienden desde los más simples a los más complejos, se denominan sistemas dinámicos caóticos. Los sistemas dinámicos caóticos atienden a las siguientes particularidades:

- Apariencia errática o aleatoria, consecuencia del carácter intrínsecamente no lineal. $^{2}$

- Dependencia sensible a las condiciones iniciales.

- Naturaleza determinista de la evolución dinámica.

La aparente complejidad dinámica puede llevar a equívocos innecesarios. Tradicionalmente, todos estos sistemas fueron etiquetados como procesos estocásticos. El descubrimiento de que sistemas deterministas muy simples son capaces de producir evoluciones extremadamente complejas supuso un cambio de paradigma en la forma de analizar todos aquellos sistemas hipotéticamente aleatorios. El hecho de que sistemas supuestamente aleatorios escondan un determinismo «camuflado» en su evolución abre un abanico de posibilidades a la hora de comprender por qué, bajo ciertas condiciones iniciales, se comportan de una determinada manera, y no de otra, y qué implicaciones estructurales y funcionales entraña su control dinámico.

Desde los orígenes de la radioastronomía, cuando, a comienzos de 1930, el ingeniero estadounidense Karl G. Jansky evidenció cómo ondas de radio procedentes de galaxias lejanas afectaban a las comunicaciones telefónicas cursadas por medio de cables trasatlánticos, o cuando los astrónomos americanos Arno A. Penzias y Robert W. Wilson identificaron, en los años 60, lo que hoy día se conoce como radiación

\footnotetext{
${ }^{2}$ Aun cuando puedan manifestarse dinámicas lineales tan complicadas como las no lineales, en dimensión finita no existe el caos lineal en sentido estricto, no así en fenómenos de dimensión infinita.
} 
cósmica de fondo, el ruido ha adquirido un papel protagonista en el análisis no lineal de sistemas dinámicos. En vez de encasillar la variabilidad errática de una señal bajo la denominación de ruido aleatorio e intentar eliminarlo, como se procedía históricamente, es el propio ruido el que se somete a un concienzudo examen, a fin de desvelar algún tipo de patrón oculto en esa aparente incertidumbre dinámica, que respalde de algún modo la causa del comportamiento observado.

Muchos sistemas fisiológicos exhiben un comportamiento presuntamente aleatorio o ruidoso, al igual que los sistemas caóticos. No obstante, la dinámica de estos últimos se rige por reglas completamente deterministas, que explican, en cierta medida, el porqué de las pautas de conducta del sistema, de ahí que sea hasta cierto punto lógico que la teoría del caos, con su batería de herramientas computacionales de análisis, haya impregnado la metodología de muchas áreas científicas, como el de la investigación clínica. Con el tiempo la supuesta presencia del caos en muchos comportamientos dinámicos se ha convertido en una regla, en lugar de una excepción, si bien es cierto que soluciones caóticas, en sentido estrictamente analítico, no son fácilmente observables a nivel experimental. A las señales adquiridas del mundo real se acoplan diferentes fuentes de ruido que enmascaran la ya de por sí compleja dinámica subyacente, lo que dificulta, en función de las proporciones de los constituyentes, la labor de separación de la componente aleatoria (ruidosa) de la componente determinista bajo estudio.

Los sistemas fisiológicos no operan de forma aislada, sino más bien de manera integral y coordinada. La proporción óptima de los constituyentes, deterministas y aleatorios, de un sistema dinámico caótico puede tener un fuerte impacto en el rendimiento que se espera del sistema. El caos ofrece toda una gama de conductas dinámicas muy diversas, lo que facilita la flexibilidad y adaptabilidad de los diferentes subsistemas fisiológicos que componen el cuerpo humano. Su aparente impredecibilidad dinámica puede tener una connotación defensiva, en el sentido de que, ante una posible amenaza o desajuste funcional, se disponga de margen de maniobra para contrarrestar un mal funcionamiento orgánico, explorando otras oportunidades dinámicas que no pongan en riesgo, al menos temporalmente, la estabilidad estructural del sistema biológico.

Por otro lado, la noción de atractor, muy recurrente en la teoría del caos, puede tener implicaciones muy interesantes desde una perspectiva fisiológica. Un atractor traza un patrón geométrico, generado por la evolución temporal de una señal biológica adquirida, una vez se ha alcanzado un régimen permanente en su evolución. El paradigma de que la dinámica caótica típicamente se desarrolla en una región del espacio de estados muy delimitada, el atractor, favorece el análisis de ciertas condiciones dinámicas singulares, supeditadas a la influencia de menos variables de estado que las que conforman el espectro de potenciales variantes dinámicas. Con más variables de estado no siempre se obtiene una mayor aleatoriedad. Muchas veces lo que prima es la velocidad de conversión entre configuraciones dinámicas (atractores), por efecto de un cambio, natural o forzado, en las condiciones paramétricas. De hecho, en diferentes estudios se concluye que la variabilidad dinámica se reduce con la edad; la variabilidad dinámica dota al sistema fisiológico de versatilidad y, por consiguiente, de habilidad intrínseca para responder y adaptarse a factores medioambientales, como situaciones de estrés. 


\subsection{Análisis no lineal de datos fisiológicos}

La descripción matemática de la realidad física ha sido una constante en la historia de la ciencia, cuya justificación un tanto esotérica fue apuntada por uno de los físicos más destacados del siglo XX, el húngaro Eugene P. Wigner (1902-1995). En cualquier caso toda formulación matemática permite cuantificar, en cierta medida, el conocimiento de que se dispone sobre un fenómeno físico, esto es, sobre su configuración estructural o funcional.

Las hipótesis que fundamentan el constructo de un modelo físico se materializan en una caracterización matemática de naturaleza axiomática. De este modo, las suposiciones conceptuales de la evolución temporal de un sistema comienzan por categorizar las pautas de comportamiento en sentido amplio, es decir, estableciéndose, por ejemplo, una distinción entre sistemas aleatorios y sistemas deterministas, o entre sistemas lineales y sistemas no lineales. En muchos casos el reconocimiento de estas clases de comportamiento se supedita al uso de herramientas analíticas apropiadas, y de su correcta caracterización depende, en el ámbito de la investigación clínica, un diagnóstico preliminar de posibles enfermedades o patologías.

En principio el análisis no lineal de datos fisiológicos tiene por cometido comprender cómo un sistema biológico funciona, dado que, conociendo las reglas que gobiernan su conducta, es todo un desafío intelectual construir un modelo analítico representativo con el que predecir, además, estados futuros. En este sentido la metereología ocupa un lugar preferente en la historia del análisis no lineal de sistemas. Hoy día al análisis no lineal de sistemas se le añade el calificativo de moderno, dado que con la aparición de los ordenadores, y su gran capacidad de cómputo, muchos modelos analíticos han dado paso a modelos numéricos más potentes, capaces de suministrar soluciones aproximadas a ecuaciones irresolubles analíticamente en el pasado, como así demostró el matemático francés Henri Poincaré.

Si en el terreno de la fundamentación teórica de las matemáticas sobresalió Henri Poincaré, en el campo de la matemática computacional despuntó el matemático húngaro John von Neumann (1903-1957), quien, aparte de sus aportaciones en materia de energía nuclear, contribuyó al diseño del primer ordenador digital, ENIAC, ${ }^{3}$ en el transcurso de la segunda guerra mundial. John von Neumann se dio cuenta de que muchos problemas de la física matemática solo podían resolverse por medio de métodos de aproximación numérica. No obstante, la investigación numérica llevada a cabo por el metereólogo americano Edward N. Lorenz (1917-2008) fue la que posibilitó el descubrimiento, el afamado efecto mariposa, de lo que actualmente se conoce como la teoría del caos.

Una vez predicho el curso futuro del comportamiento de un sistema, se suscita, de forma natural, la posibilidad de alterar su rumbo, esto es, de controlarlo para llevarlo a un determinado estado, o alejarlo de estados no aconsejables. Las intenciones ligadas a la comprensión, predicción y control, pueden extrapolarse a las fases de diagnóstico y tratamiento de la práctica clínica. Comprender cómo funciona un subsistema fisiológico y cómo puede verse alterado su comportamiento en presencia

\footnotetext{
${ }^{3}$ Acrónimo de Electronic Numerical Integrator and Computer. Si bien es considerado el primer ordenador de propósito general, compite en honores con el Atanasoff Berry Computer (ABC), atribuido al ingeniero estadounidense John V. Atanasoff (1903-1995).
} 
de una patología atienden a un posible procedimiento de diagnóstico, siendo el tratamiento un mecanismo de control que intenta restaurar la configuración funcional del subsistema atendido [Shelhamer, 2007]. Los primeros indicios de la aplicación de técnicas no lineales al análisis evolutivo de una dolencia se encuentran en el ámbito de la epidemiología, mientras que es la cardiología el área que ha recibido más atención, tanto en relación con la evaluación diagnóstica como en términos de control funcional.

Las ciencias de la vida, en general, y la fisiología, en particular, no hubieran experimentado el progreso que hoy por hoy han alcanzado sin los avances que paralelamente se introducían en los primeros principios de la física. Los algoritmos computacionales derivados de un análisis no lineal de los datos tienen un carácter genérico, si bien pueden sugerir variaciones cuando se aplican en el ámbito específico de las señales biológicas, donde las generalizaciones no se admiten caprichosamente, dadas las complejas interdependencias presentes en los sistemas fisiológicos.

Un subconjunto de variables cuyos valores cambian con el tiempo define un sistema dinámico. Estas variables, en conjunto, caracterizan el estado del sistema en un instante de tiempo determinado, de ahí que reciban el nombre de variables de estado. Dado que los valores de estas variables de estado cambian con el tiempo, su variación describe una trayectoria en un espacio, el espacio de estados. En la concepción de un modelo dinámico un aspecto clave, y de gran trascendencia, es la selección de las variables que ha de incluir el modelo. De hecho, muchos modelos son perfeccionados conforme se incorporan nuevas variables al modelo originario. Además de las variables, la interacción entre las variables recopiladas encierra la esencia del modelo dinámico. Estas interacciones se describen en términos de ecuaciones diferenciales, en tiempo continuo, o, en términos de ecuaciones en diferencias, si el tiempo es discreto. Si se conocen las ecuaciones el problema está resuelto; se dispone de todo el conocimiento necesario para describir cómo se comporta el sistema y qué evolución se espera de él. El inconveniente al que se enfrenta la investigación fisiológica es que habitualmente se desconocen las ecuaciones que rigen el comportamiento del sistema fisiológico de interés, y de lo único de que se dispone es de ciertas variables biológicas susceptibles de medición.

Una cuestión muy relevante, y de difícil resolución, implica idear algún mecanismo que permita relacionar las mediciones con las variables de estado y, a partir de ellas, crear un modelo dinámico que caracterice su evolución temporal. En la misma línea la mayoría de los sistemas fisiológicos cuentan con infinidad de variables de estado acopladas entre sí, y no todas ellas guardan idéntica importancia. De esta manera, a fin de simplificar el análisis, se suele reducir el número de variables de estado consideradas, en función de su supuesta contribución a la dinámica general del sistema. Es más, a veces es necesario establecer como constantes algunas variables de estado, en función de lo que se esté analizando, de forma que, en la escala de tiempo convenida, solo intervengan ciertas variables en la dinámica subyacente.

En definitiva la definición de las variables de estado, y la medición de sus valores, resulta crucial en la modelización y el análisis de los sistemas dinámicos. Eso sí, en el contexto de las señales biológicas no siempre es posible identificar y medir todas las variables que intervienen en el sistema fisiológico bajo estudio. Por tanto, no en pocos casos se dispone de las observaciones de una sola variable de estado y ha de 
recurrirse a técnicas de reconstrucción del espacio de estados sobre la base de la única información disponible.

El análisis no lineal de datos fisiológicos no cuenta con una larga tradición, si bien se dispone de un sinfín de herramientas software, provenientes de otras disciplinas científicas que recibieron más atención en el pasado. Estas aplicaciones software permiten ejecutar de forma inmediata algoritmos matemáticos ideados para calcular propiedades, supuestamente inherentes a la dinámica subyacente, a partir de la sucesión temporal adquirida de una medición biológica. En este sentido lo importante no es tanto reducir la sucesión temporal a un simple número, que caracterice una cualidad estructural o funcional del sistema, como sí, conocida la fundamentación matemática de los algoritmos software, comprender, sobre la base de los resultados y de las conclusiones derivadas, el funcionamiento del sistema fisiológico de interés. Solo de esta manera podrá abordarse, con cierta solvencia, una de las fases más críticas y arduas, a la par que desatendida, del análisis de datos, la identificación del sistema dinámico que generó los datos disponibles.

La finalidad de la identificación de un sistema dinámico estriba en construir, a partir de la sucesión temporal de que se dispone, junto con magnitudes cuantitativas extraídas de los propios datos, un modelo matemático que describa fielmente el sistema dinámico que generó los datos [Nelles, 2013]. Los modelos matemáticos más sencillos, los modelos de regresión lineal, se circunscriben al ámbito de la dinámica lineal, en la que una vez conocidas las variables de entrada, las variables de salida y las variables de estado, se determinan los coeficientes de las ecuaciones diferenciales, o en diferencias, lineales que las relacionan. Por ejemplo, en un modelo de media móvil (modelo MA), la salida actual depende únicamente de entradas pasadas; en cambio en un modelo autorregresivo (modelo AR), la salida actual depende solamente de las salidas pasadas. Un modelo combinado (modelo ARMA) agrupa ambas predisposiciones. En cualquier caso la determinación del modelo es más un arte que una ciencia, pues el planteamiento está más sujeto a la intuición y a la experiencia del diseñador que a un procedimiento sistemático estándar, máxime cuando el modelo debe contemplar la presencia de ruido, lo que convierte al modelo en un modelo probabilístico. Desde una perspectiva ingenieril el modelo de ruido que suele utilizarse en modelos lineales es el ruido blanco gaussiano aditivo, representado por variables aleatorias independientes e idénticamente distribuidas, que reproducen la aportación de ruido blanco gaussiano a cada muestra de la sucesión temporal. Así, en el proceso estocástico conviene diferenciar la componente determinista de la componente aleatoria, esta última dependiente linealmente de los valores aleatorios precedentes [Ross, 2007, Gardiner, 2004].

La especificación de un modelo no lineal es una tarea mucho más peliaguda, dado que se abren infinitas posibilidades en el repertorio de funciones no lineales que pudieran modelizar un sistema no lineal, como logaritmos, funciones trigonométricas, polinomios, exponenciales, etc. Una primera fase del diseño del modelo obliga a precisar el modelo de sistema (funciones no lineales) que mejor reproduce el fenómeno físico, así como el orden del sistema (número de parámetros) [Palit and Popovic, 2006]. Esta etapa es crucial, y, sin embargo, no recibe la atención que merece, en parte debido a su excesiva dificultad, pues alude a uno de los aspectos esenciales de la ciencia, la descripción de lo que sucede. En una segunda fase, de naturaleza más 
sistemática, se ajustan los parámetros del modelo.

En el proceso de selección del modelo de sistema no lineal han ido apareciendo con el tiempo diferentes alternativas [Judd and Mees, 1995], desde los modelos clásicos, derivados de los primeros principios de la mecánica clásica, hasta los modelos fenomenológicos, concebidos a partir de las mediciones adquiridas del fenómeno físico. En el ámbito experimental normalmente se dispone de una sucesión temporal con mediciones u observaciones de algunas variables físicas del sistema, por lo que, a priori, se dispone de un conocimiento muy limitado de las leyes que gobiernan la evolución dinámica del sistema, esto es, con un conocimiento incompleto del sistema, supeditado solamente a un número finito de observaciones, la solución al cometido inverso de plantear un modelo general, capaz de suministrar las observaciones adquiridas, no es única. La ambigüiedad surgida puede resolverse parcialmente si se introducen restricciones al procedimiento de diseño, como optar por el modelo de sistema más simple que se ajuste de forma consistente a las observaciones, sin descuidar factores como su tratabilidad computacional.

Los modelos basados en las observaciones de una sucesión temporal presentan inconvenientes consustanciales a la propia concepción metodológica, puesto que los parámetros del modelo no suelen tener significación física, sino más bien estadística, es decir, el modelo propuesto genera datos con las mismas propiedades estadísticas que las observaciones. En cualquier caso si los parámetros del modelo escogido presentan algún tipo de correspondencia física o fenomenológica se dice que el modelo es paramétrico; en cambio si no puede establecerse ninguna equivalencia entre los parámetros y el fenómeno físico observado se habla de que el modelo es no paramétrico.

La ciencia pretende, sobre todo, una descripción exacta de lo que sucede, de forma que una explicación rigurosa de los sucesos se convierta en un modo de medir hechos, y, a su vez, que estas medidas de los hechos permitan predecir eventos futuros, lo que confiere cierto dominio o control sobre los fenómenos examinados. Para que un modelo matemático sea fiel reflejo de la realidad no solo debe «funcionar», interpretando este funcionamiento como su capacidad para generar datos con sentido, sino que debe abarcar, en principio, todas las posibilidades funcionales a las que debería responder en función de los estímulos externos a los que estaría sometido posiblemente el sistema. Esta cuestión está todavía abierta, dado que, a diferencia de un entorno controlado, como pueda ser un laboratorio, en el mundo real los sistemas físicos no se desenvuelven de forma aislada, sino que de una $u$ otra forma interaccionan entre ellos, por múltiples motivos, lo que dificulta sobremanera la confección de un modelo global, no solo en términos computacionales sino más bien en términos estrictamente descriptivos.

Con objeto de simplificar las labores de modelización se suele optar por una $\mathrm{u}$ otra clase de modelo en virtud del propósito de la construcción del mismo y, no en pocas circunstancias, del conocimiento que del sistema físico bajo estudio se tiene. En algunos casos el modelo de sistema se dedica a producir datos artificialmente, a fin de estimar invariantes geométricas o dinámicas compartidas con el sistema originario, como dimensiones, entropías, exponentes de Lyapunov, etc. En otros casos el modelo de sistema actúa como un modelo predictivo, en el que suele prevalecer su capacidad predictiva a corto plazo, explotándose de forma óptima aproximaciones lineales en un 
entorno local de la dinámica del sistema físico. En líneas generales no se dispone de una receta algorítmica que posibilite ultimar metódicamente un modelo de sistema que obedezca con rigor a las reglas de comportamiento del sistema físico de interés para todas las posibles configuraciones dinámicas.

La experiencia del diseñador juega un papel preponderante en el problema de identificación de un sistema, en concreto, en áreas tan complejas como la fisiología [Marmarelis, 2012], y, posiblemente, sea el conocimiento acumulado que del sistema físico atesore, lo que haga que se decante por una estructura particular de modelo de sistema. En este punto cabe resaltar que algunas técnicas de análisis no lineal, que evalúan el comportamiento dinámico del sistema, pueden sugerir preliminarmente opciones en cuanto a la clase de modelo más apropiada, como que el sistema sea lineal o no lineal, determinista o estocástico, estacionario o no estacionario, etc. Desde los modelos más clásicos, como las representaciones polinómicas de Volterra y Wiener [Volterra, 1959, Wiener, 1966, Rugh, 1981], hasta los más recientes, como los algoritmos genéticos [Palit and Popovic, 2006], que, basados en el poder de cómputo actual, exploran concurrentemente diferentes configuraciones que respalden el comportamiento global del sistema físico, han ido emergiendo nuevos métodos, cada vez más sofisticados, como las redes neuronales, que exploran la sutil estructura no lineal de muchos sistemas físicos, conforme a funciones de base que aproximen localmente su dinámica [Bishop, 2006].

Se admite comúnmente que los sistemas fisiológicos son sistemas no lineales, de ahí su supuesta complejidad. En consecuencia los datos fisiológicos podrían beneficiarse, en principio, de las técnicas desarrolladas en el marco de la teoría de los sistemas dinámicos. Sin embargo, la mayoría de los métodos de análisis no lineal de sucesiones temporales se fundamentan en conceptos derivados del caos determinista, debido a la riqueza dinámica que exhiben los sistemas caóticos; particularmente, las herramientas de análisis se han centrado en la evaluación del caos de bajas dimensiones. La contrariedad surge cuando se aplican estas técnicas de análisis a los sistemas fisiológicos, que, al igual que el cuerpo humano en su conjunto, no son deterministas.

La teoría de los sistemas dinámicos se ocupa del estudio de los sistemas cerrados, esto es, sistemas que no interactúan con el mundo circundante. En cambio los sistemas fisiológicos son sistemas abiertos y, por consiguiente, interaccionan con el mundo exterior, al que, en la jerga termodinámica, se denomina foco calórico (heat $b a t h)$. En el contexto de los sistemas fisiológicos el foco calórico simboliza la interacción compleja entre los diferentes subsistemas fisiológicos que conforman el cuerpo humano, y, dado que se desconoce el acoplamiento mutuo, adopta a priori un número infinito de grados de libertad [Kantz and Schreiber, 1995]. En el mejor de los casos la influencia exterior se considera simplemente ruido desacoplado que, en principio, no afecta a la dinámica intrínseca del sistema fisiológico bajo estudio, a pesar de que pueda introducir «inconvenientes estadísticos». En cualquier otro caso los cambios del entorno circundante, en teoría extensibles a cualquier escala temporal, introducen no estacionariedad en el registro de los datos durante el tiempo que dure el experimento. Además, el impacto de los cambios no tiene por qué restringirse a los parámetros del sistema, sino que incluso pueden concernir a los estados del sistema, es decir, estos cambios pueden resolverse como ruido dinámico.

A tenor de estas evidencias los datos fisiológicos han de estimarse como datos no 
lineales con un alto grado de estocasticidad, lo que los sitúa en un marco bastante alejado del popular caos determinista. A modo de código deontológico el sentido común reclama una extremada prudencia cuando se interpretan los resultados recabados de la aplicación de las diferentes técnicas de análisis no lineal a las señales biológicas. La cautela debe nutrirse de indicadores cualitativos en lugar de conclusiones numéricas precipitadas, dada la complejidad biológica que encierran las ciencias de la vida desde tiempos ignotos.

\subsubsection{Ley de evolución dinámica}

En el pasado un comportamiento irregular o errático en una sucesión temporal era considerado como ruido superpuesto a la dinámica real, y, como tal, era prioritario eliminarlo, a fin de resolver la supuesta evolución lineal del sistema físico bajo estudio. Hoy día, con la eclosión de la teoría del caos y la progresiva incorporación de nuevas herramientas de análisis no lineal, lo que tradicionalmente era calificado como ruido se ha convertido en una fuente de información dinámica sin precedentes, que contribuye, de otra manera, a desmitificar la prevalencia de los sistemas lineales, en beneficio de los cada vez más extendidos sistemas no lineales.

En principio un comportamiento irregular se circunscribe exclusivamente a una dinámica no lineal. La evolución temporal de un sistema lineal autónomo, con $d$ grados de libertad, $\mathbf{x}(t)=\left[x_{1}(t), x_{2}(t), \ldots, x_{d}(t)\right]$, obedece a

$$
\frac{\mathrm{d} \mathbf{x}(t)}{\mathrm{d} t}=\mathbf{E} \cdot \mathbf{x}(t),
$$

donde $\mathbf{E}$ representa una matriz de evolución de dimensión $d \times d$. Las diferentes soluciones de la ecuación (5.3) identifican las órbitas que puede trazar el sistema en un espacio $d$-dimensional. Aquellas direcciones que experimentan una contracción denotan direcciones estables y se caracterizan por los autovectores de $\mathbf{E}$ cuyos autovalores presentan una parte real negativa, mientras que aquellas direcciones que se extienden hasta el infinito indican direcciones inestables y se corresponden con los autovectores de $\mathbf{E}$ cuyos autovalores retienen una parte real positiva. En este último caso cualquier aproximación lineal a la dinámica del sistema físico es errónea, y, en consecuencia, ha de recurrirse a ecuaciones dinámicas no lineales [Abarbanel et al., 1993, Eckmann and Ruelle, 1985].

Si se conocen a priori las ecuaciones que rigen la evolución dinámica del sistema, la aplicación de las herramientas de análisis es directa. En la práctica el principal inconveniente radica en que únicamente se dispone de una sucesión temporal que recoge los valores que adoptan los diversos observables del sistema físico, típicamente un solo observable escalar, adquiridos mediante algún procedimiento de medición. En este sentido la información de partida es, ya de por sí, incompleta. Además, no es infrecuente que los datos medidos estén contaminados de ruido. Por consiguiente, el primer cometido al que ha de enfrentarse el experimentador es separar la información útil de toda posible interferencia, esto es, separar la señal de información del ruido, con independencia de que el sistema físico subyacente sea lineal o no lineal. Una primera tentativa estándar implica hacer uso de la transformada de Fourier para evaluar el contenido frecuencial de la señal temporal capturada. El espectro de 
potencia evidencia la contribución de todos los posibles períodos, desde el período de muestreo hasta el tiempo total que abarca la sucesión temporal. Si las bajas frecuencias detentan excesiva potencia, y no existe continuidad en el rango frecuencial alrededor de $f=0$, debe considerarse la sucesión temporal no estacionaria, dado que las componentes frecuenciales correspondientes presentan pocas oscilaciones durante el período de observación.

En sistemas lineales las señales generadas muestran frecuencias (modos de operación) claramente diferenciadas del ruido de fondo, con un espectro de potencia de banda ancha representativo. De este modo, es relativamente sencillo separar la señal útil del ruido acoplado, dada la discrepancia existente entre los contenidos espectrales de la fuente de señal útil y el espectro de las diferentes fuentes de ruido que puedan imbricarse. En sistemas no lineales, como los sistemas caóticos, el espectro de la señal útil exhibe características frecuenciales muy similares a las atribuidas al ruido, dificultándose la labor de extracción de las componentes frecuenciales útiles, de ahí que la transformada de Fourier no sea la mejor opción a este respecto. Las diferentes alternativas propuestas, agrupadas bajo la denominación de técnicas de reducción de ruido, descansan en una misma idea; se presupone que el atractor en que se desenvuelve la dinámica caótica es de baja dimensión, a diferencia del ruido, que, al ser de naturaleza aleatoria, intenta ocupar todo el espacio disponible y, por tanto, es de dimensión infinita.

Una vez eliminado el ruido de la señal, ha de reconstruirse el espacio de estados en que se desarrolla la dinámica presente en la sucesión temporal adquirida, siendo un factor crítico la estacionariedad de los datos [Schreiber, 1999]. En sentido estricto la estacionariedad implica que las probabilidades condicionales de evolución entre estados no dependen del tiempo. En el contexto de las sucesiones temporales, en las que solamente se cuenta con una realización del proceso físico de longitud limitada, se desconocen las probabilidades de transición entre estados y han de estimarse a partir de los datos mismos. Así, no es raro que las probabilidades calculadas se vean sometidas a fluctuaciones estadísticas que difieren entre realizaciones y que deterioran en cierto modo la estabilidad probabilística, máxime en presencia de intermitencia en el sistema bajo estudio. ${ }^{4}$ Con objeto de que las técnicas de análisis operen adecuadamente resulta a menudo imprescindible que los parámetros del sistema físico no cambien de manera significativa durante el tiempo que conlleva la medición de las observaciones. En ocasiones se opta por considerar ventanas de tiempo lo bastante cortas como para que los parámetros del sistema no dispongan de tiempo suficiente para cambiar y lo bastante largas (suficientemente muestreadas) para que la dinámica del sistema exhiba todas las escalas de tiempo implícitas. A tenor del espectro de potencia, la escala temporal característica coincide con el recíproco de la frecuencia más baja que contiene un cierto porcentaje de la potencia total de la señal.

En señales biológicas la estacionariedad es la excepción y no la regla, dado que sus parámetros dependen del tiempo, y es muy difícil confirmar la estacionariedad de una sucesión temporal de longitud finita. Es más, en ocasiones los cambios temporales de los parámetros (ausencia de estacionariedad) revelan información muy

\footnotetext{
${ }^{4}$ La intermitencia, remítase a la página 93, comprende un fenómeno muy habitual en la dinámica no lineal, por el que se suceden de forma alterna fases regulares y caóticas, cuya aparición y duración es impredecible [Pomeau and Manneville, 1980].
} 
interesante sobre la dinámica inherente a un proceso físico. Por tanto, el propósito del análisis inclinará la balanza de una posible solución de compromiso entre la resolución temporal (evaluación de múltiples escalas de tiempo) y la relevancia estadística del parámetro analizado. Por ejemplo, operar con la derivada de una sucesión temporal, en vez de con los datos en bruto, introduce estacionariedad en el sistema, en detrimento del impacto del ruido acoplado, que se verá incrementado, poniendo en riesgo el posible determinismo del sistema dinámico.

\subsubsection{Cómo evaluar la no linealidad}

Lo primero que debería emprenderse cuando uno se enfrenta a una sucesión temporal es examinar los datos desde diferentes perspectivas. La forma más natural comprende una inspección visual de los datos, una vez representados en función del tiempo, con objeto de descubrir, de un vistazo, posibles problemas de estacionariedad, escalas de tiempo, e incluso eventos atípicos. De este modo, pueden seleccionarse aquellos intervalos de la sucesión temporal que se antojan estacionarios. Acto seguido se proyectarían varios diagramas de fases bidimensionales, $x(t)$ vs. $x(t-\tau)$, para diversos valores de $\tau$, a fin de detectar posibles estructuras en los datos, indicio de presumible determinismo, o simetrías que pueden sugerir transformaciones en los datos que faciliten su análisis, como incorporar más datos a la sucesión temporal simplemente añadiendo sus valores simétricos.

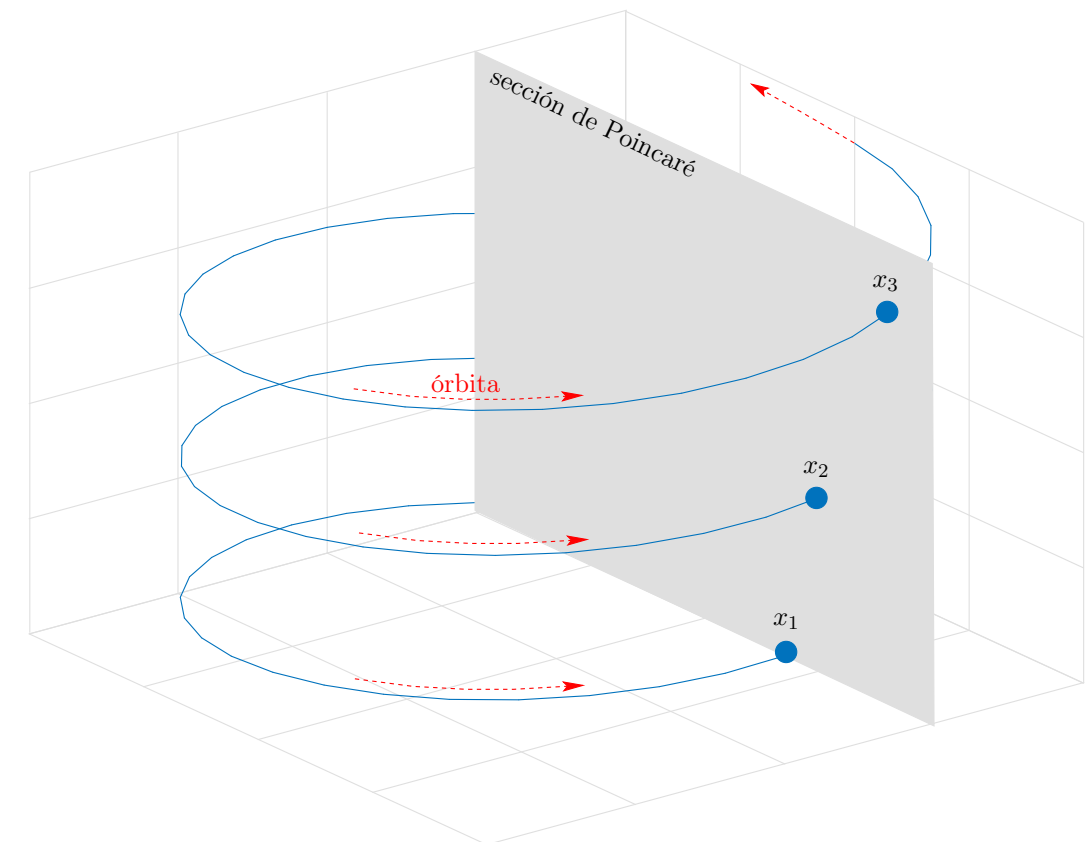

Figura 5.2: Esbozo de una sección de Poincaré.

Un mecanismo para transformar un flujo dinámico (tiempo continuo $t$ ), de dimensión $d$, en un mapa (tiempo discreto $n$ ), de dimensión $d-1$, consiste en orientar una hipersuperficie transversalmente a la dirección del flujo, llamada sección de Poincaré [Wiggins, 2003], y contabilizar las intersecciones del flujo con la hipersuperficie 
en la dirección especificada, como ilustra la figura 5.2. Los cruces son consignados como iteraciones del mapa, que recibe el nombre de mapa de Poincaré o mapa de retorno (return map), si bien el tiempo discreto $n$ no guarda proporción alguna con el tiempo continuo $t$, dado que el tiempo que transcurre entre dos sucesivas intersecciones es variable, ${ }^{5}$ dependiendo de las trayectorias descritas por el sistema dinámico en el espacio de reconstrucción y de la hipersuperficie seleccionada. Para que el mapa de Poincaré suministre una adecuada información dinámica, la hipersuperficie ha de orientarse oportunamente, de forma que registre convenientemente la recurrencia de las trayectorias, y puedan revelarse patrones geométricos que permitan caracterizar el tipo de atractor presente, si fuere el caso. En definitiva la sección de Poincaré proporciona información sobre la estructura interna del atractor, o, dicho de otro modo, sobre cómo el atractor desarrolla su dinámica localmente, ignorándose la configuración geométrica global. En cierto sentido la sección de Poincaré, si bien tiene restricciones operativas, no funcionales - solo admite gráficamente atractores de dimensión igual o inferior a tres-, constituye un modo alternativo de intentar descubrir visualmente la presencia de determinismo en los datos, conforme a las estructuras «definidas» que describen los puntos que interceptan la hipersuperficie [Stewart, 1989].

\subsubsection{Evaluación gráfica}

Un primer método para detectar no estacionariedad recurre a la estimación de momentos estadísticos en diferentes segmentos temporales de la sucesión temporal. Si la diferencia entre los momentos estadísticos es significativa se concluye que la sucesión temporal es no estacionaria [Witt et al., 1998]. En sistemas lineales normalmente se admite como prueba de estacionariedad el cumplimiento de homogeneidad estadística hasta los momentos de segundo orden, la media y la varianza (estacionariedad débil). En sistemas no lineales este método no es muy fiable y obliga a buscar indicadores no lineales de estacionariedad, como la distribución de probabilidad presente en el espacio de estados, siempre que se excluyan las correlaciones temporales entre vecinos [Isliker and Kurths, 1993]. Dicho de otro modo, la presencia de no estacionariedad en los datos introduce una tendencia a que puntos próximos en el espacio también lo sean en el tiempo. Una herramienta gráfica muy útil para examinar las distancias temporales y geométricas entre los diferentes estados que describen el fenómeno observado es el gráfico de recurrencia (recurrence plot) [Eckmann et al., 1987, Marwan et al., 2007], como se observa en la figura 5.3. Un par de instantes de tiempo $t_{1}, t_{2}$ conforman una recurrencia si los vectores de estado $\mathbf{x}_{t_{1}} \mathrm{y} \mathrm{x}_{t_{2}}$ están muy próximos, situados a una distancia igual o menor que un valor de resolución $\varepsilon$. La recurrencia, una suerte de repetición aproximada de un estado, queda establecida como

$$
r_{\varepsilon}\left(t_{1}, t_{2}\right)=\left(1-\delta_{t_{1} t_{2}}\right) K_{\varepsilon}\left(\left\|\mathbf{x}_{t_{1}}-\mathbf{x}_{t_{2}}\right\|\right),
$$

donde $\delta_{t_{1} t_{2}}$ representa una delta de Kronecker, y $K_{\varepsilon}$ un kernel que suele coincidir con la función escalón de Heaviside, a saber, $K_{\varepsilon}(r)=\Theta(\varepsilon-r)$. Dado que los vectores $\mathbf{x}_{t}$

\footnotetext{
${ }^{5}$ En caso de que el tiempo entre intersecciones coincida, como sucede en sistemas periódicos o cuasiperiódicos, se dice que el mapa es estroboscópico.
} 
son el resultado de un proceso de reconstrucción de un espacio de estados, el gráfico de recurrencia especifica una matriz simétrica $\mathbf{R}_{\varepsilon}$ de orden $N_{v}$, con $N_{v}=N-(m-1) \tau$, siendo $N$ el número de puntos de la sucesión temporal y $N_{v}$ el número de vectores reconstruidos, cada uno de ellos de dimensión $m$ y con una separación entre sus componentes de $\tau$ unidades de tiempo. La matriz simétrica $\mathbf{R}_{\varepsilon}$ recibe el nombre de matriz de recurrencia y el valor de cada una de sus entradas, según la ecuación (5.4), determina si los vectores son recurrentes, $R_{i j}=1$, o no son recurrentes $R_{i j}=0$. La distribución espacial de los puntos que configuran la matriz de recurrencia aporta información muy valiosa sobre la dinámica del sistema físico. Un motivo por el que no se manifiesta recurrencia y, por tanto, no se aprecian segmentos de línea de corta longitud dispuestos paralelamente a la diagonal principal, reside en la posibilidad de que la sucesión temporal adquirida se corresponda con un transitorio, y la condición inicial que generó la trayectoria actual no forme parte del atractor, donde habría de revelarse la recurrencia dinámica. Si el gráfico de recurrencia muestra muchos puntos aislados en los segmentos de línea paralelos a la diagonal principal, es indicativo de proximidades casuales, fruto de la presencia de ruido en los datos o de muchos falsos vecinos, cuando se opera con una dimensión de reconstrucción insuficiente. En líneas generales, si predominan los puntos aislados en el gráfico de recurrencia, lo más probable es que el sistema físico no sea determinista o, en su defecto, con el valor de $\varepsilon$ prefijado, el determinismo sea de carácter débil. La estacionariedad de una sucesión temporal precisa que los segmentos de línea se distribuyan de manera uniforme en el gráfico de recurrencia.

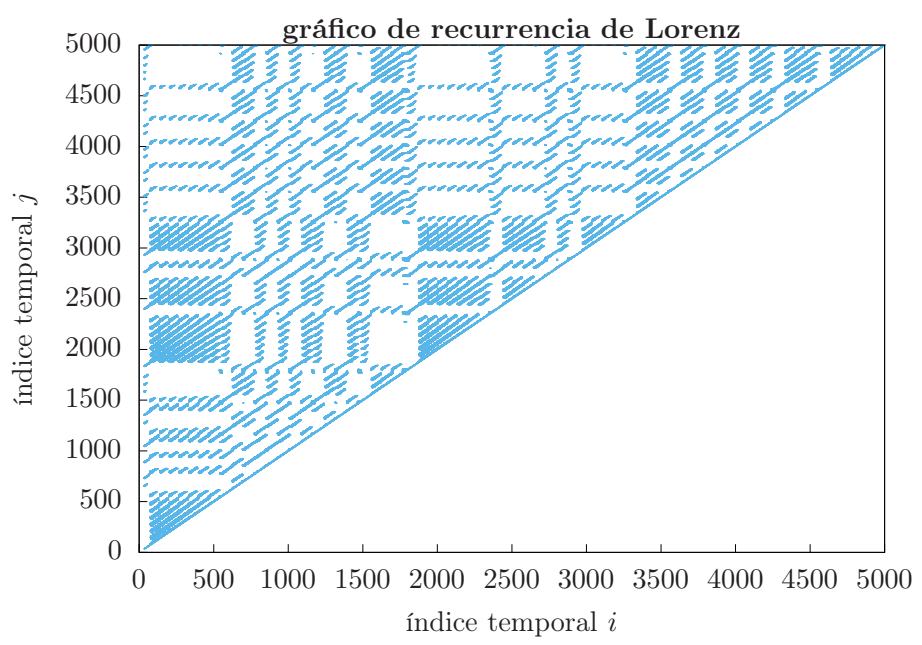

Figura 5.3: Gráfico de recurrencia del atractor de Lorenz para la variable dinámica $x$. La representación gráfica obedece a $m=3, \tau=16$ y $r=5$.

Si los parámetros $m$ y $\tau$ son seleccionados correctamente, según lo avalado en el capítulo 3, en el caso límite $\varepsilon \rightarrow 0, N \rightarrow \infty$, el gráfico de recurrencia se aproxima a los parámetros que varían con el tiempo. Del gráfico de recurrencia es difícil extraer cuantitativamente la variación temporal que experimentan los parámetros, si bien sí puede desprenderse el número de parámetros fluctuantes así como las escalas de 
tiempo en las que operan las fluctuaciones. En este sentido resulta muy ventajoso promediar el número de recurrencias entre ventanas temporales, por medio de la integral de correlación cruzada $C_{X Y}(\varepsilon)$ [Kantz, 1994], esto es,

$$
C_{X Y}\left(\varepsilon, w, t_{1}, t_{2}\right)=\frac{1}{w^{2}-\sum_{i=1}^{w} \sum_{j=1}^{w} \delta_{t_{1}+i t_{2}+j}} \sum_{i=1}^{w} \sum_{j=1}^{w} r_{\varepsilon}\left(t_{1}+i, t_{2}+j\right),
$$

donde $X, Y$ identifican segmentos temporales, de longitud $w$, de la misma sucesión temporal, uno comenzando en $t_{1}$ y el otro en $t_{2}$. La integral de correlación cruzada sirve de base para definir medidas relativas de disimilitud entre diferentes procesos físicos o, incluso, entre diferentes características, dinámicas o geométricas, dentro de un mismo proceso físico [Manuca and Savit, 1996]. A fin de evaluar la no estacionariedad de una sucesión temporal, el error promedio de un predictor no lineal localmente constante puede reescribirse en términos de recurrencias como

$$
\gamma\left(\varepsilon, w, t_{1}, t_{2}\right)^{2}=\sum_{i=1}^{w}\left(\hat{\mathbf{x}}_{t_{1}+i+1}-\mathbf{x}_{t_{1}+i+1}\right)^{2},
$$

donde la estimación de la predicción $\hat{\mathbf{x}}_{t_{1}+i+1}$ deriva del promedio de un entorno de tamaño $\varepsilon$, a saber,

$$
\hat{\mathbf{x}}_{t_{1}+i+1}=\left\{\begin{array}{l}
\frac{1}{\alpha} \sum_{j=1}^{w} r_{\varepsilon}\left(t_{1}+i, t_{2}+j\right) \mathbf{x}_{t_{2}+j+1}, \quad \alpha>0, \\
\frac{1}{w} \sum_{j=1}^{w} \mathbf{x}_{t_{2}+j+1}, \quad \alpha=0,
\end{array}\right.
$$

donde $\alpha=\sum_{j=1}^{w} r_{\varepsilon}\left(t_{1}+i, t_{2}+j\right)$ representa el número de vecinos de $\mathbf{x}_{t_{1}+i}$ situados a una distancia menor o igual que $\varepsilon$. La variación del error de predicción en función del segmento temporal es un indicio de la ausencia de estacionariedad en la sucesión temporal. Dicho con otras palabras, dados dos segmentos temporales $i$ y $j$, uno de ellos, p. ej., el segmento $i$, se emplea como entrenador del predictor, mientras que el otro segmento, el segmento $j$, sirve como banco de pruebas para efectuar el test de fiabilidad del predictor. El error cuadrático medio cruzado entre el valor predicho por el predictor, sobre la base del segmento $i$, y el valor real del segmento $j$, sirve de referencia para contrastar las diferentes dinámicas de los segmentos y así desvelar también la ausencia de estacionariedad en los datos [Schreiber and Schmitz, 1997]. El error de predicción $\gamma\left(\varepsilon, w, t_{1}, t_{2}\right)$ puede emplearse como una matriz de disimilitud entre segmentos temporales, para su posterior tratamiento mediante un algoritmo de agrupamiento, de forma que las distancias de los segmentos a los grupos o clusters reflejen la variación temporal de los parámetros.

El promedio total de $\sum_{i, j} r_{\varepsilon}\left(t_{i}, t_{j}\right)$ equivale a la integral de correlación $C(\varepsilon)$. En la práctica la estimación de la dimensión de correlación $D_{2}$ adolece de la correlación temporal, $\mathrm{y}$, por consiguiente, ha de prescindirse de aquellos puntos vecinos que

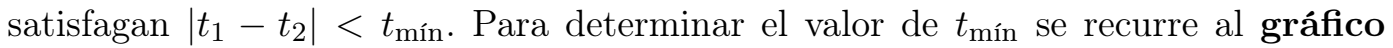
de separación espacio-temporal [Provenzale et al., 1992]. En este gráfico, como 
ilustra la figura 5.4, se muestran las líneas de contorno $C(\varepsilon, \Delta n)$ que representan el porcentaje de puntos vecinos, para diferentes valores de $\varepsilon$, respecto a la separación temporal $\Delta n$ entre ellos, siendo

$$
C(\varepsilon, \Delta n)=\frac{1}{N_{v}-\Delta n} \sum_{t=1+\Delta n}^{N_{v}} r_{\varepsilon}(t, t-\Delta n) .
$$

Las líneas de contorno de la ecuación (5.8) crecen conforme a $\Delta n$ hasta un valor en el que estas se saturan y mantienen un valor aproximadamente constante, ${ }^{6}$ con independencia del valor que adopte $\Delta n$. El valor mínimo de $\Delta n$ para el que se nivelan las líneas de contorno constituye un valor adecuado de $t_{\text {mín}}$.

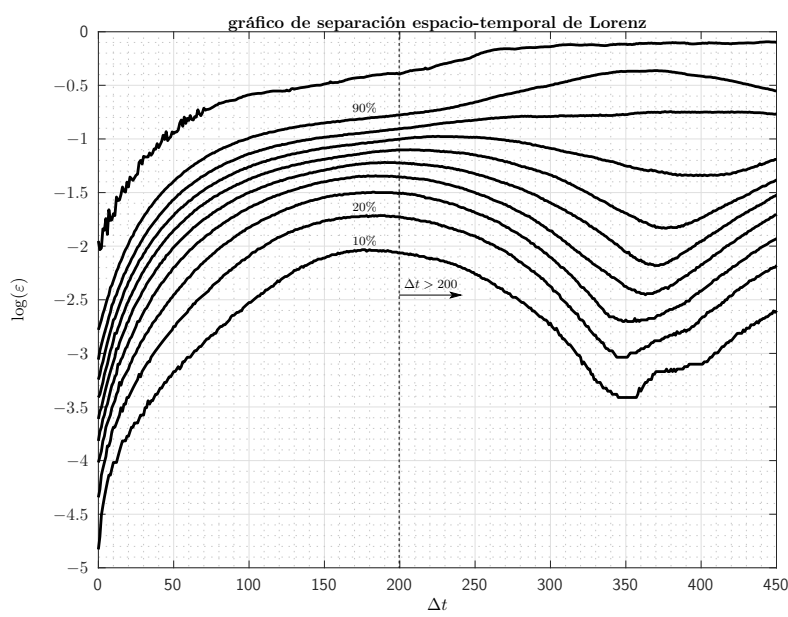

Figura 5.4: Gráfico de separación espacio-temporal del atractor de Lorenz para la variable dinámica $x$. Con $m=3$ y $\tau=16$, se muestran las líneas de contorno que representan, para una separación espacial $\varepsilon$ y una separación temporal $\Delta t$, las fracciones de puntos (en \%) $\mathbf{x}_{i} \in \mathbb{R}^{m}$ encontrados.

\subsubsection{Test estadístico}

En procesos estacionarios el teorema ergódico posibilita reemplazar el promedio de un ensamble (conjunto de realizaciones de un mismo experimento) por un promedio temporal, más asequible cuando se dispone de una única realización del sistema físico, o sea, cuando se dispone, como es habitual, de una única sucesión temporal. El promedio temporal se obtiene por segmentación de la sucesión temporal en ventanas de tiempo, procediéndose a estimar, para cada ventana, un estadístico de interés. Si el proceso físico es estacionario el modelo matemático que lo caracteriza ha de ser autónomo, no dependiente del tiempo.

En los sistemas dinámicos no es infrecuente que un cambio en alguno de sus parámetros desencadene una alteración sustancial en su dinámica, o, al menos, en

\footnotetext{
${ }^{6}$ En diversas situaciones el gráfico de separación espacio-temporal experimenta oscilaciones. En estos casos el valor mínimo de $t_{\text {mín }}$ ha de ser superior al primer ciclo de oscilación, evitándose el primer intervalo temporal en el que el porcentaje de vecinos crece debido a la correlación dinámica entre ellos.
} 
alguna de las propiedades que caracterizan la complejidad del proceso, como la dimensión del atractor, ${ }^{7}$ los exponentes de Lyapunov, la entropía, etc., en un modo impredecible. De alguna manera, algún parámetro del sistema ha alcanzado un punto crítico, un punto de crisis, que traslada drásticamente la evolución dinámica del sistema hacia otro atractor completamente diferente. En este sentido cualquier medida de la complejidad del sistema queda desvirtuada, pues no refleja la invariancia de las propiedades que caracterizan los diferentes modos de operación (regímenes dinámicos) que puede desarrollar un proceso físico. Por tanto, se requiere un test estadístico apropiado que posibilite distinguir diferentes estructuras o regímenes dinámicos, o bien permita extraer información cualitativa de la dinámica del sistema físico en presencia de datos no estacionarios.

Dado que un sistema dinámico puede contener estructuras lineales y no lineales, un primer test estadístico debe confirmar la presencia de elementos no lineales en la dinámica presente, atendiendo a los datos disponibles, antes de someterlos a un exhaustivo análisis no lineal. Diversas evidencias ponen de manifiesto que procesos estocásticos lineales son capaces de producir estructuras, desde principios lineales, aparentemente tan complejas como las generadas por factores no lineales. No hay que olvidar que si los datos son lineales el espectro de potencia puede proporcionar, por sí solo, una descripción estadística completa del proceso físico subyacente.

Además, la presunta irregularidad (aperiodicidad) de los datos puede provenir de sistemas no lineales deterministas o, por el contrario, son las componentes aleatorias que se acoplan a un sistema lineal, inclusive, las distorsiones no lineales que introduce el procedimiento de medición (no linealidad estática) o las fluctuaciones en los parámetros, las inductoras del comportamiento errático que se manifiesta en la dinámica de los datos. Únicamente señales no lineales deterministas quedan caracterizadas por atractores de dimensión finita, exponentes de Lyapunov positivos y errores de predicción no lineal pequeños. En teoría cualquiera de estos indicadores era signo inequívoco de determinismo no lineal, si bien con el tiempo se ha demostrado que puede confundirse con configuraciones lineales, por lo que ha de recurrirse a algún contraste de hipótesis que, sobre la base de un formalismo más consolidado, avale la procedencia real de los datos, a saber, si los datos son producidos por un proceso lineal o no lineal. En este sentido el estadístico sometido al test de hipótesis ha de ser consistente con la prueba de significación estadística.

El test estadístico que mejor se adapta al análisis no lineal de sucesiones temporales es el método de los datos sustitutos (surrogate data), una variación del método de Montecarlo [Theiler et al., 1992, Theiler and Prichard, 1997]. Primeramente se define una hipótesis nula que se someterá a valoración positiva (aceptación) o negativa (rechazo) en función de los datos disponibles. En términos de no linealidad una hipótesis nula muy habitual presupone que los datos proceden de un proceso estocástico lineal gaussiano. A continuación se especifica el nivel de significación $\alpha$ de la prueba estadística. Un valor común es $\alpha=0,05$, lo que significa que se exige un $95 \%$ de aciertos en el contraste de hipótesis, o, lo que es lo mismo, se admite la posibilidad de que en un $5 \%$ de los casos se rechace falsamente la hipótesis nula. La probabilidad de rechazo de la hipótesis nula no ha de exceder el nivel de signi-

\footnotetext{
${ }^{7}$ Una magnitud estadística muy sensible a la no estacionariedad de los datos es la dimensión de correlación.
} 
ficación estadística. Con objeto de completar la prueba estadística deben generarse sucesiones temporales «sustitutas» que guarden cierta relación con la sucesión temporal original, de forma que el vínculo establecido sea congruente con la hipótesis nula que ha de contrastarse. Finalmente, se computa el estadístico $\lambda$ de la prueba de contraste de hipótesis tanto para la sucesión temporal original, $\lambda_{\mathrm{do}}$, como para las diferentes sucesiones temporales sustitutas $\lambda_{\mathrm{ds}_{i}}$, y se determina la probabilidad de que el estadístico $\lambda_{\text {do }}$ obtenido de la sucesión temporal original se haya extraído de la distribución del estadístico, conforme a las sucesiones temporales sustitutas creadas. Por ejemplo, si se asume que el estadístico, evaluado en los datos sustitutos, sigue una distribución gaussiana de media $\bar{\lambda}$ y desviación típica $\sigma$, se admitiría como verdadera la hipótesis nula, con un nivel de significación del $95 \%$, si $\left|\lambda_{\text {do }}-\bar{\lambda}\right|<2 \sigma$. Normalmente no se puede conocer a priori que el estadístico siga una distribución gaussiana, ni siquiera es posible confeccionar su distribución de probabilidad con sucesiones temporales de longitud finita, por lo que se recomienda aplicar un test de rangos en la prueba de contraste de hipótesis. De esta manera, se generan $1 / \alpha$ sucesiones temporales sustitutas que satisfagan la hipótesis nula. Se rechaza la hipótesis nula cuando el estadístico de la sucesión temporal original es menor que todos los estadísticos de los datos sustitutos, en un test unilateral, o mayor o menor que todos los estadísticos de los datos sustitutos, si se recurre a un test bilateral.

A fin de que el test estadístico sea robusto, se precisa que el estadístico elegido pueda detectar fielmente desviaciones en los datos respecto a la hipótesis nula considerada, y, por otro lado, que el ensamble, conformado por las diferentes sucesiones temporales sustitutas, represente fidedignamente la hipótesis nula. La capacidad discriminatoria del estadístico elegido constituye un factor clave en el proceso de su elección, ${ }^{8}$ puesto que comporta una medida del grado de desviación de los datos en relación con la hipótesis nula. En aplicaciones clínicas y fisiológicas, dado el virtual carácter crítico de las evaluaciones diagnósticas, se aconsejan estadísticos que difieran lo máximo posible entre los datos originales y los datos sustitutos, pero con varianzas mínimas, acrecentándose así el poder discriminante del test de hipótesis. Otra cuestión nada desdeñable repara en el error estadístico del estimador que evalúa la propiedad que se desea contrastar. En el ámbito del análisis no lineal de sucesiones temporales la estimación de los diferentes estadísticos se supedita a diversos factores que trascienden incluso a los propios datos, como el algoritmo que posibilita su cómputo o la asignación paramétrica que precede a la implementación del algoritmo, en muchos casos sujeta a criterios subjetivos. Con estos precedentes, es prácticamente imposible proporcionar un error estadístico, si bien el método de Montecarlo podría solventar el inconveniente, suministrando una probabilidad de error. En cualquier caso, en materia de discriminación estadística no es tan importante la verosimilitud del estadístico como sí su mayor o menor varianza, que incide directamente en el poder del test. A este respecto cabe subrayar el impacto que los parámetros de la reconstrucción ocasionan en las varianzas de los estadísticos, y, por consiguiente, en la resolución discriminatoria.

Considerando un test de no linealidad, un estadístico de la no linealidad presente en una sucesión temporal que merece especial atención, por su poder discriminante,

\footnotetext{
${ }^{8} \mathrm{El}$ poder discriminante $1-\beta$ de una prueba estadística identifica la probabilidad de que se rechace una hipótesis nula cuando en verdad es falsa.
} 
en comparación con otros marcadores más clásicos, derivados del espectro de potencia, apunta a una variante de la autocorrelación, que mide la asimetría de una sucesión temporal ante una inversión temporal [Diks et al., 1995], esto es,

$$
\phi^{\operatorname{inv}}(\tau)=\frac{\sum_{n=\tau+1}^{N}\left(x_{n}-x_{n-\tau}\right)^{3}}{\left[\sum_{n=\tau+1}^{N}\left(x_{n}-x_{n-\tau}\right)^{2}\right]^{3 / 2}} .
$$

En un proceso estocástico lineal cualquier estadístico siempre satisface la simetría temporal. Otros estadísticos, basados en errores de predicción o en magnitudes derivadas de la suma de correlación, también muestran resultados satisfactorios [Schreiber and Schmitz, 1997]. Cabe señalar que un test de no linealidad no sugiere necesariamente determinismo, cuya huella queda consignada en una dinámica de baja dimensión o en la capacidad predictiva del modelo que reproduce de forma aproximada el sistema físico. Con sucesiones temporales finitas, las estimaciones de los diferentes estadísticos adolecen inevitablemente de fluctuaciones estadísticas, cuyo efecto se refuerza con la varianza del estadístico, muy sensible a la no estacionariedad de los datos disponibles, lo que reduce el poder discriminante de la prueba de test. Un test estadístico de no linealidad incapaz de rechazar la hipótesis nula o bien indica que los datos no presentan no linealidad o bien que el test no puede detectar la no linealidad, debido, en muchas ocasiones, a su escaso poder discriminante. Cuanto más estable sea el estadístico empleado, aun en perjuicio de la veracidad de la propiedad que se pretenda medir, mejores prestaciones se obtendrán en una prueba de significación estadística, es decir, menos falsos rechazos introducirá la prueba. Además, la presencia de no estacionariedad en los datos puede causar un incremento en el número de falsos positivos, a saber, eventos singulares pueden interpretarse como desviaciones de la hipótesis de linealidad. Eso sí, el hecho de que se empleen estadísticos propios de la teoría del caos para corroborar la no linealidad de los datos no significa que, en caso de que el test de no linealidad sea positivo, la dinámica sea caótica.

La validez del test estadístico basado en los datos sustitutos descansa en la apropiada generación del ensamble, conformado por sucesiones temporales a las que, aun siendo aleatorias, se las impone cierta estructura, conforme a la hipótesis nula especificada. Si el proceso no se lleva a cabo correctamente pueden manifestarse desviaciones espurias que, si son detectadas por el test, se interpretarían erróneamente como un signo de no linealidad. Uno de los problemas del test de no linealidad estriba en la delimitación de la hipótesis nula. Dado que normalmente se establece como hipótesis nula que los datos proceden de un sistema lineal, se insinúa implícitamente que la hipótesis nula es compuesta, esto es, abarca toda una extensa clase de procesos lineales, lo que obliga a redefinir el problema en términos de propiedades de los procesos físicos, en lugar de las ecuaciones que rigen su dinámica. Con este nuevo enfoque, el procedimiento para crear sucesiones temporales sustitutas recibe el nombre de realizaciones restringidas (constrained realisations) [Theiler and Prichard, 1996].

La especificación de la hipótesis nula de un proceso estocástico lineal estacionario con entradas gaussianas puede entonces reformularse estableciéndose que toda la configuración dinámica del proceso queda completamente caracterizada por los mo- 
mentos estadísticos de primer y segundo orden, la media y la varianza, así como por la función de autocorrelación. Esto significa que puede obtenerse una muestra aleatoria confeccionándose una sucesión temporal con las mismas propiedades de segundo orden que los datos originales, siendo en todo lo demás aleatoria. Cuando las propiedades lineales se describen mediante las magnitudes cuadráticas de los coeficientes de Fourier (periodograma de la transformada de Fourier), esto se traduce en que los datos sustitutos constan de números aleatorios correlados con el mismo espectro de potencia que los datos originales. Sea $\left\{x_{n}\right\}_{n=1}^{N}$ la sucesión temporal inicial, con $N$ observaciones. La transformada discreta de Fourier (FFT) asciende a

$$
\tilde{x}_{k}=\frac{1}{\sqrt{N}} \sum_{n=1}^{N} x_{n} e^{i 2 \pi n k / N} .
$$

Si se multiplican los coeficientes complejos $\tilde{x}_{k}$ por fases aleatorias, es decir,

$$
\tilde{x}_{k}^{\prime}=\tilde{x}_{k} e^{i \phi_{k}},
$$

con la variable $\phi_{k}$ distribuida uniformemente en el intervalo semiabierto $[0,2 \pi)$. A fin de que la transformada inversa de Fourier suministre datos reales, se precisa que $\phi_{N-k}=-\phi_{k}$. Así, la transformada inversa de $\tilde{x}_{k}^{\prime}$ obedece a

$$
x_{n}^{\prime}=\frac{1}{\sqrt{N}} \sum_{k=1}^{N} \tilde{x}_{k}^{\prime} e^{-i 2 \pi n k / N} .
$$

La sucesión temporal $\left\{x_{n}^{\prime}\right\}_{n=1}^{N}$ contiene números aleatorios con el espectro de potencia prescrito. Diferentes realizaciones de $\phi_{k}$ generan nuevos datos sustitutos. El proceso de aleatorización de las fases preserva la distribución gaussiana de los datos sustitutos, al menos en el límite asintótico $N \rightarrow \infty$.

La desviación más natural de esta hipótesis nula es que los datos no sigan una distribución gaussiana. Una posible conjetura del mecanismo que pudiera provocar la desviación es que la salida del proceso estocástico lineal estacionario con entradas gaussianas haya sido distorsionada por una función de medición $h$ monótona, instantánea e independiente del tiempo,

$$
x_{n}=h\left(s_{n}\right),
$$

con $s_{n}$ identificando un proceso estocástico lineal estacionario, con entradas gaussianas, que, en su concepción más simple, atiende a un modelo autorregresivo de media móvil (ARMA),

$$
s_{n}=\sum_{i=1}^{M_{\mathrm{AR}}} a_{i} s_{n-i}+\sum_{j=1}^{M_{\mathrm{MA}}} \eta_{j} s_{n-j},
$$

siendo $\eta_{j}$ números aleatorios independientes extraídos de un proceso gaussiano de media cero y varianza unidad. La función de medición $h$ hace que la distribución de probabilidad de las observaciones, además del espectro de potencia, sea desconocida. Una aproximación habitual es invertir $h$ empíricamente, reescalando los valores de $x_{n}$ de forma que $s_{n}$ se ajuste a una distribución gaussiana, esto es, $\hat{s}_{n}=\hat{h}^{-1}\left(x_{n}\right)$. 
Sobre los valores de $\hat{s}_{n}$ se efectúa la aleatorización de la fase, generándose los datos sustitutos $\hat{s}_{n}^{\prime}$. Estos datos son reescalados de nuevo hacia atrás para obtener los datos $x_{n}^{\prime}$. La distribución de los datos sustitutos $x_{n}^{\prime}$ coincidirá con la de los datos originales $x_{n}$, si bien el espectro de potencia diferirá, dado que se manifestarán ligeras diferencias entre las magnitudes de $s_{n}$ y de $\hat{s}_{n}$, al estimar la función $h$, desconocida de antemano. Un reajuste en amplitud de la FFT de los datos sustitutos, una suerte de «pulido» de los datos sustitutos, sería suficiente si no fuera porque, en presencia de distorsiones no lineales, junto con correlaciones entre los datos, se incrementa el número de falsos rechazos de la hipótesis nula. En este sentido se ha propuesto una implementación algorítmica que pretende adaptar más estrechamente los datos sustitutos al oportuno espectro de potencia, sobre la base de un filtrado de los datos similar a la estructura funcional de un filtro de Wiener [Schreiber and Schmitz, 1996]. Así, si $\left|\tilde{x}_{k}\right|$ denota las magnitudes de los coeficientes de Fourier de los datos originales, para filtrar los datos sustitutos, se computan las FFT de los datos sustitutos, $\tilde{x}_{k}^{\prime}, \mathrm{y}$ se reemplazan sus valores por

$$
\tilde{x}_{k}^{\prime \prime}=\frac{\tilde{x}_{k}^{\prime}\left|\tilde{x}_{k}\right|}{\left|\tilde{x}_{k}^{\prime}\right|},
$$

procediéndose con el cálculo de la transformada inversa de Fourier; aplicando de manera reiterada este esquema, el espectro de potencia termina convergiendo a su verdadera distribución si $N$ es lo bastante grande.

En suma, para que el test de no linealidad procure un buen rendimiento se requiere que los datos sustitutos presenten el mismo periodograma y la misma distribución probabilística que los datos originales, de ahí que se recurra a algún tipo de filtrado de los datos sustitutos, a fin de corregir las posibles desviaciones detectadas. No obstante, no solo es necesario que las amplitudes de los coeficientes de Fourier de los datos originales y de los datos sustitutos coincidan, sino también la estructura de la autocovarianza,

$$
C(\tau)=\frac{1}{N-\tau} \sum_{n=\tau+1}^{N} x_{n} x_{n-\tau},
$$

pues esta determina las amplitudes de los coeficientes de Fourier, siempre que la sucesión temporal se corresponda con un período de la secuencia, que se repetiría indefinidamente cada $N$ muestras temporales, es decir, para que se conserven las amplitudes de los coeficientes de Fourier de los datos, la función de autocovarianza ha de ser periódica, lo que significa que los extremos de las sucesiones temporales deben concordar. En caso contrario se introducen artefactos en los datos sustitutos que pueden llevar a falsos rechazos en el test de no linealidad. Una técnica muy simple que subsana, en cierta medida, el contratiempo pasa por seleccionar un intervalo de la sucesión temporal en la que los extremos sean aproximadamente equivalentes, si bien es probable que siga apreciándose un desajuste en la fase de los puntos extremos. Es por esto que se ha ideado un método de aleatorización de los datos, basado en permutaciones de los datos originales, capaz de lograr, en sentido estricto, la periodicidad de la función de autocovarianza [Schreiber, 1998]. El algoritmo de aleatorización de los datos sustitutos permite crear sucesiones temporales aleatorias 
con una correcta distribución de los valores, donde se impone una restricción adicional, que la función de autocovarianza de los datos sustitutos $C^{\prime}(\tau)$ ha de ser la misma que la de los datos originales $C(\tau)$. La discrepancia de las autocovarianzas se expresa en términos de una función objetivo,

$$
E^{(q)}=\left[\sum_{\tau=0}^{N-1}\left|C^{\prime}(\tau)-C(\tau)\right|^{q}\right]^{1 / q} .
$$

La función objetivo $E^{(q)}\left(\sigma\left(\left\{x_{n}\right\}\right)\right)$ se minimiza, por medio del método de recocido simulado (simulated annealing) [Vidal, 1993], atendiendo a todas las permutaciones $\sigma$ de la sucesión temporal original $\left\{x_{n}\right\}$, a fin de obtener la aleatorización idónea de los datos originales. Por intercambiar pares en las permutaciones se adquieren diferentes configuraciones. Este método de minimización (optimización) combinatorial es muy flexible, pues se presta a restricciones arbitrarias en las características que han de reunir los datos sustitutos, en relación con los datos de partida, facilitándose de alguna manera, con la incorporación de restricciones, la definición formal de una hipótesis nula.

\subsubsection{Dinámica simbólica}

A veces, más que un análisis cuantitativo de los datos, se persigue un examen cualitativo de los mismos. En consecuencia resulta muy útil reducir la sucesión temporal a una representación simbólica, a fin de extraer características relevantes de la dinámica subyacente, como la detección de saltos cualitativos en la dinámica del proceso debido a cambios repentinos en algún parámetro del sistema. La dinámica simbólica asigna etiquetas (símbolos) a los patrones específicos encontrados en la sucesión temporal, procediéndose a estudiar de forma cuantitativa la sucesión de símbolos en lugar de la sucesión temporal originaria [Crutchfield and Packard, 1982, Destexhe et al., 1989]. De alguna manera la dinámica simbólica comporta un análisis más genérico (coarse-grained) de los datos disponibles que, convertidos en símbolos, pueden igualmente prestarse a minuciosas y fidedignas medidas de complejidad siempre que la codificación simbólica responda a una razonable interpretación fenomenológica [Mischaikow et al., 1999].

\subsection{Un ejemplo de señal biológica: la señal PPG}

La monitorización de la salud mediante métodos no invasivos ha atraído la atención de los especialistas médicos desde hace ya algunos años, puesto que les permite adelantar diagnósticos preliminares sobre posibles disfunciones patológicas, ya sean estas crónicas o transitorias [Noah et al., 2018]. Diversas evidencias científicas corroboran la relación existente entre las señales biológicas generadas por el sistema cardiovascular y el estado de salud del sujeto, a partir de indicadores evaluados principalmente sobre la morfología de las mismas [Elgendi et al., 2018]. En este sentido la técnica denominada fotopletismografía, o, en inglés photoplethysmogram (PPG), ha adquirido especial trascendencia en los últimos tiempos por su especial facilidad para adquirir, con la ayuda de un oxímetro de pulso o pulsioxímetro, información 
fisiológica asociada al sistema cardiovascular. Un oxímetro de pulso consta de un emisor de luz y de un fotodetector que recoge y registra (pulso o señal PPG) la dispersión que experimenta un haz de luz cuando este atraviesa un tejido humano, típicamente el presente en un dedo, o, inclusive, en el lóbulo de una oreja. Dada la sencillez de su acomodación no invasiva, además de su bajo coste, un oxímetro de pulso resulta muy útil en aplicaciones biomédicas para entornos clínicos [Allen, 2007] y deportivos [Jr. et al., 2016].

Si bien el estudio del sistema cardiovascular y de la circulación sanguínea se remonta a tiempos inmemoriales, el incremento espectacular de las enfermedades cardiovasculares en la última década, sobre todo en los países más industrializados, reivindica una investigación más exhaustiva de los procesos físicos subyacentes a la dinámica cardíaca y circulatoria. En el caso particular de España, según el último informe elaborado por el Instituto Nacional de Estadística en 2016, bajo el auspicio de la Sociedad Española de Cardiología, las enfermedades cardiovasculares son la primera causa de mortalidad, seguidas de las enfermedades tumorales y de las enfermedades respiratorias [Sociedad Española de Cardiología, 2016]. Los factores de riesgo que se barajan incluyen elementos relacionados con el estilo de vida que, con carácter general, predomina en la población durante un período determinado. Más allá de los hábitos saludables, véanse el descanso, el ejercicio, y la alimentación, los aspectos socioeconómicos, como la competitividad laboral, las condiciones en el trabajo y los cánones sociales vigentes, están pasando factura entre los más vulnerables a niveles físico y psicológico. Es por todo ello que una monitorización continua del estado de salud en que se encuentra un individuo permitiría reducir hasta cierto punto los factores de riesgo que, en ausencia de contramedidas, podrían conducir a situaciones del todo irreversibles. Para lograr una notoria efectividad en la supervisión del paciente es preciso disponer de un profundo conocimiento sobre la dinámica del sistema cardiovascular, accediendo a los indicadores fisiológicos adecuados.

Con la irrupción de las nuevas tecnologías y su frenético desarrollo ha sido posible disponer de dispositivos electrónicos, con gran capacidad de cómputo y a precios asequibles, capaces de adquirir y analizar en tiempo real señales biológicas, lo que ha contribuido a que actualmente toda una gama de equipos de monitorización de la salud sea una parte integral del equipamiento médico en entornos hospitalarios [Tamura et al., 2014], y, en muchos casos, accesible al público en general [Nam and Nam, 2017, Blasco and Peris-Lopez, 2018, Ridder et al., 2018]. Es por esta razón que en la práctica clínica ya es rutinario monitorizar las constantes vitales, como la frecuencia cardíaca, la frecuencia respiratoria, la tensión arterial, la saturación de oxígeno en sangre, la temperatura, etc., de manera permanente.

Desde hace más de una década, y con los índices de mortalidad por enfermedades cardiovasculares en aumento, se han renovado esfuerzos por comprender las señales biológicas, a fin de extraer características de ellas que identifiquen en un alto grado las condiciones idóneas de salud y sus posibles desviaciones. De esta manera podrían establecerse indicadores asociados a diferentes patologías, como sucede con la señal PPG, que anticipen su gravedad en función de las causas que las suscitaron [Elgendi et al., 2018, Allen et al., 2005, Eerikäinen et al., 2018, Lee et al., 2018, Reguig and Reguig, 2017, Wu et al., 2017, Djawad et al., 2017, Elgendi et al., 2015b, Kohjitani et al., 2014, Elgendi, 2012a, Allen, 2007, ichiro Komatsu et al., 2003]. No obstante, 
y a diferencia del electrocardiograma (ECG) [Sen and McGill, 2017] o del electroencefalograma (EEG) [Bachmann et al., 2018, Sato et al., 2013], pocos han sido los estudios, particularmente en el ámbito de la fotopletismografía, que, en vez de extraer características morfológicas de las señales biológicas, aspiren a evaluar el comportamiento dinámico, sobre la base de la teoría del caos, del proceso físico responsable de su generación [Sviridova and Sakai, 2015b, Sviridova, 2015, Moraes et al., 2018], y no tanto a valorar otros aspectos colaterales, véase el procesamiento de señales biológicas, como la determinación de ventanas de tiempo estacionarias [Riley et al., 2012]. En un futuro próximo la eficacia de las aplicaciones de monitorización de la salud descansará en el conocimiento que se atesore sobre la dinámica de los procesos fisiológicos subyacentes.

La revolución tecnológica del siglo pasado en el campo de la bioingeniería no solo ha permitido acceder más fácilmente a las señales biológicas sino también ha posibilitado compilar un registro inmenso de señales de alta resolución, aptas para un análisis intensivo, gracias a las altas velocidades computacionales de los ordenadores actuales. En el contexto particular del sistema cardiovascular las señales biológicas que con mayor frecuencia se miden, por su trascendencia médica y porque se avienen a una adquisición no invasiva, comprenden el electrocardiograma (ECG), la tensión arterial (TA) y la onda de pulso cardiovascular proporcionada por la PPG. De estas señales resulta factible extraer información relativa a la salud del paciente, como, por ejemplo, la frecuencia cardíaca, por medio de la variabilidad del pulso cardíaco, o la saturación de oxígeno en sangre, por medio de la PPG. De cualquier manera, toda la información recuperada de las diferentes señales procede del mismo sistema fisiológico, el sistema cardiovascular, una estructura de la que participan múltiples subsistemas, acoplados de un modo complejo [Stefanovska, 1999], si bien las diversas señales biológicas pueden suministrar diferentes perspectivas de una misma dinámica.

Este estudio únicamente atiende la onda de pulso cardiovascular adquirida mediante fotopletismografía o photoplethysmogram (PPG), porque, en esencia, esta técnica de monitorización óptica se ha convertido en una herramienta habitual de diagnóstico de algunas enfermedades, como la hipertensión y la enfermedad de las arterias coronarias, debido a la simplicidad electrónica, la relación coste beneficio, la facilidad de adquisición de señales, y, primordialmente, por su carácter no invasivo [Moraes et al., 2018]. La técnica PPG se remonta a 1937, cuando el fisiólogo estadounidense Alrick Hertzman ideó un fotopletismógrafo fotoeléctrico que describió como un dispositivo que se sirve de la absorción de la luz por parte de un tejido transiluminado para estimar el volumen sanguíneo [Hertzman, 1937]. A diferencia de otras señales biológicas, que requieren, para su medición, un equipamiento voluminoso, los fotopletismógrafos recientes, también denominados pulsioxímetros, cada vez son más pequeños, ligeros y con una marcada tendencia a comercializarse como dispositivos inalámbricos [Castaneda et al., 2018, Peart et al., 2018]. Un aspecto importante de la técnica PPG radica en su escasa sensibilidad a la ubicación de los sensores, dotando a la fotopletismografía de versatilidad para su aplicación en infinidad de áreas, como las áreas de la salud, el deporte, o la industria agroalimentaria [Allen, 2007, Elgendi, 2012a, Sviridova and Sakai, 2015a]. A pesar de los posibles artefactos que se acoplan en el proceso de medición de la onda de pulso cardiovascular, por medio de esta es posible adquirir información relacionada con el ritmo cardíaco [Schäfer and Vagedes, 
2013] y la frecuencia respiratoria [Charlton et al., 2017], si bien aún se desconoce del todo cómo se integran sobre una misma señal, la onda de pulso cardiovascular, las respuestas de los diferentes subsistemas fisiológicos que conforman el sistema cardiovascular, lo que ha alentado esta investigación, fundamentalmente en términos de análisis dinámico, con el propósito de que algún día pudiera desembocar en nuevas y más flexibles aplicaciones de monitorización y control de la salud de la población. Hasta ahora los parámetros cardiovasculares detectados mediante la tecnología PPG incluyen la frecuencia cardíaca, la saturación de oxígeno en sangre, la tensión arterial, la evaluación de la rigidez arterial y la velocidad de la onda de pulso, entre otros. De alguna manera la señal PPG incluye información sobre el proceso hemodinámico, la hemorreología y el estado tisular del sistema de microcirculación periférica en el cuerpo humano [Liang et al., 2018].

\subsubsection{La onda de pulso cardiovascular y sus características}

La técnica PPG, acrónimo de Photoplethysmogram, permite registrar variaciones del volumen sanguíneo que se producen con cada latido del corazón [Elgendi, 2012a]. Si bien los diversos dispositivos PPG, también denominados fotopletismógrafos o pulsioxímetros, miden los cambios del volumen sanguíneo de diferente forma, en general son muy económicos y eficaces; constan de un emisor de luz y de un fotodetector que capta la onda de pulso cardiovascular que se propaga por el cuerpo humano. Esta onda de pulso, o pulso PPG, describe el movimiento, en cierta medida ondulatorio (ver figura 5.5), que experimenta la sangre en los vasos sanguíneos, en su trayecto desde el corazón a las extremidades.

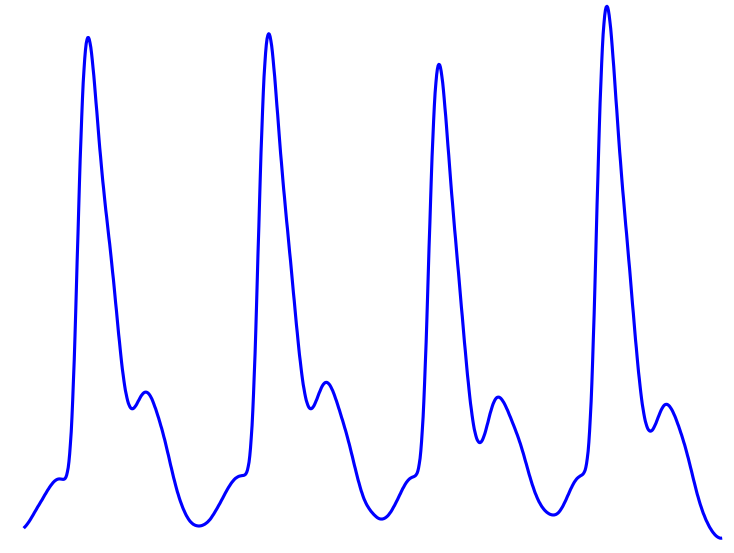

Figura 5.5: Forma de onda de una señal PPG típica.

Normalmente se dirige una luz infrarroja invisible ${ }^{9}$ a un tejido (p. ej., la piel) y se captura, con un fotodetector, la cantidad de luz retrodispersada, esto es, la variación de la intensidad de la luz, relacionada estrechamente con la variación del volumen

\footnotetext{
${ }^{9}$ En los dispositivos más modernos se emplea un diodo LED que opera en la región espectral del infrarrojo cercano (Near-Infrared Region o, expresado con siglas, NIR), concretamente en la banda comprendida entre 0,8 y $1 \mu \mathrm{m}$. De esta manera se favorece la medición del flujo sanguíneo en tejidos más profundos, no solo a nivel cutáneo [Sviridova, 2015].
} 
sanguíneo; de hecho, una disminución del volumen sanguíneo implica un aumento de la intensidad de la luz. Los fotodetectores suelen presentar dos configuraciones: modo transmisión y modo reflectante [Webster, 1997]. En el primer modo de operación el tejido se sitúa entre el transmisor y el fotodetector, de forma que este recoja la luz refractada por el tejido. En el segundo modo la luz incide sobre el tejido, de modo que el fotodetector, yuxtapuesto al emisor, se encarga de captar la luz reflejada por el tejido. El modo transmisión es muy común en los pulsioxímetros de los hospitales, donde el dedo del paciente es pinzado por el fotopletismógrafo, como ilustra la figura 5.6 .

La forma de onda de un pulso PPG se distorsiona por los efectos que los latidos del corazón, la hemodinámica y las condiciones fisiológicas de las arteriolas y/o vénulas tienen sobre su perfil característico. Por tanto, el análisis de la morfología del pulso PPG puede suministrar información muy valiosa de los sistemas circulatorio y respiratorio. No obstante, la calidad del pulso PPG está condicionada por factores propios del individuo sometido a la prueba de medición de la señal [Moço et al., 2018, Chan et al., 2013], como las propiedades de su piel, su temperatura, el caudal en sangre, la saturación de oxígeno en sangre, etc., y por factores externos, derivados de las condiciones ambientales del entorno en que se lleva a cabo la medición del pulso PPG, como el ruido de alta frecuencia que se acopla a la electrónica de adquisición o artefactos debidos al movimiento del sujeto mientras se efectúa la captura del pulso, entre otros. En cualquier caso todos estos factores contaminan, en mayor o menor medida, y de forma aditiva, la señal PPG, lo que podría dificultar el proceso de extracción correcta de características del pulso PPG, y, por consiguiente, podría conducir a un diagnóstico erróneo de posibles dolencias. De esta manera, resulta imprescindible un apropiado preprocesamiento de la señal PPG antes de proceder con la obtención de características fisiológicas [Akar et al., 2013].

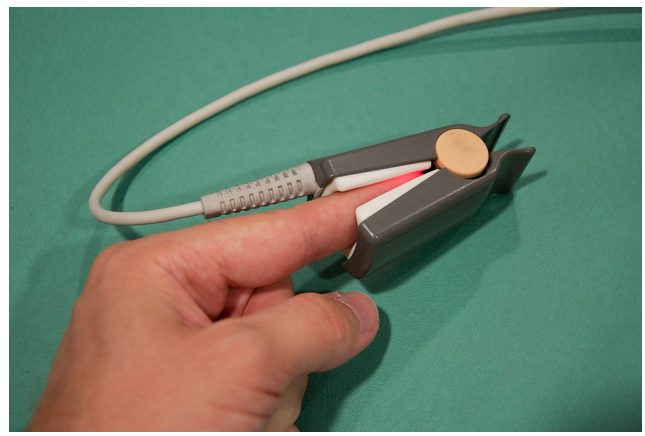

Figura 5.6: Pinzamiento de un dedo con el pletismógrafo a fin de capturar la señal PPG de un individuo [imagen disponible públicamente en flickr y adquirida el 30 de octubre de 2018].

Aunque el pulso PPG guarda cierto parecido con la señal que describe un pulso de la presión arterial, encierra diferencias significativas. El flujo sanguíneo periférico se ve afectado por la actividad simpática y por variaciones de la temperatura, dado que la vasculatura periférica, p. ej., la yema del dedo, experimenta una vasoconstricción (estrechamiento de los vasos sanguíneos) ante ciertas reacciones fisicoquímicas. Estos fenómenos introducen errores sustanciales cuando se pretende establecer relaciones 
entre la forma de onda del pulso PPG y las propiedades de las arterias principales.

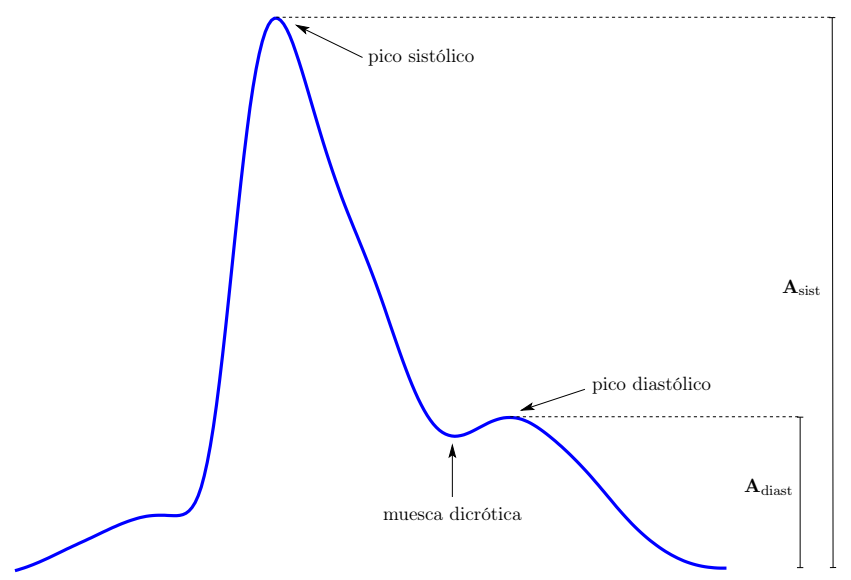

(a) Picos sistólico y diastólico.

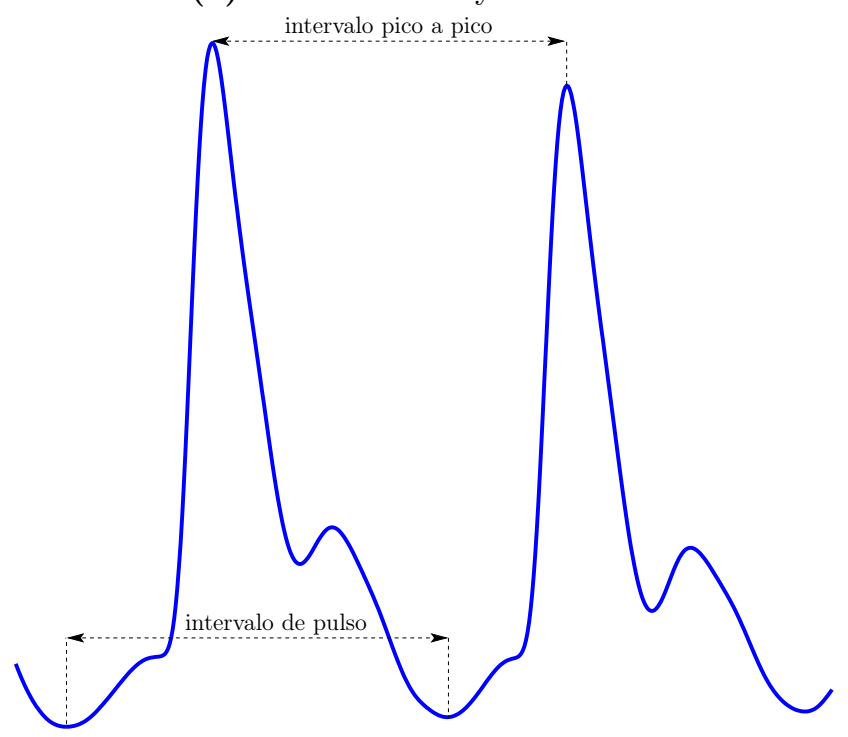

(b) Intervalo pico a pico.

Figura 5.7: Parámetros característicos del pulso PPG. En (a) la amplitud del pico sistólico se denota con el símbolo $\mathbf{A}_{\text {sist }}$ y la amplitud del pico diastólico con el distintivo $\mathbf{A}_{\text {diast }}$. En (b) el intervalo pico a pico entre dos pulsos consecutivos de la señal PPG.

La apariencia de un ciclo o pulso PPG se representa en la figura 5.7a. El pulso PPG consta de dos fases diferenciadas: la fase anacrótica se corresponde con el flanco de subida del pulso; la fase catacrótica se identifica con el flanco de bajada. La fase anacrótica se refiere al proceso sistólico, mientras que la fase catacrótica concierne al proceso diastólico y a las reflexiones de onda desde el sistema periférico [Elgendi, 2012a]. En individuos con arterias sanas se aprecia una muesca dicrótica en la fase catacrótica. La amplitud sistólica constituye un indicador de variaciones en el volumen sanguíneo. La amplitud sistólica es directamente proporcional a la distensibilidad vascular local, de ahí que potencialmente guarde cierta relación con 
la presión sanguínea.

El intervalo pico a pico comprende el intervalo de tiempo que transcurre entre dos picos sistólicos consecutivos (ver figura 5.7b). El intervalo R-R del pulso ECG está íntimamente ligado al intervalo pico a pico de la segunda derivada de la señal PPG, también denominada señal APG (Acceleration PlethysmoGram); en ambos casos especifica un ciclo cardíaco completo. El intervalo de tiempo comprendido entre el comienzo y el final de un pulso PPG se denomina intervalo de pulso. Este indicador suele emplearse en lugar del intervalo pico a pico cuando los picos diastólicos son más fáciles de detectar que los picos sistólicos. Ya sea con el intervalo pico a pico o con el intervalo de pulso, resulta factible calcular la señal HRV (Heart Rate Variability) a partir de la señal PPG [Schäfer and Vagedes, 2013, Gil et al., 2010].

Esta metodología sencilla, no invasiva y de bajo coste ofrece beneficios relevantes en lo referente a la asistencia sanitaria (primeros auxilios) o, incluso, estudios sobre el estado de salud de la población, pues posibilita un diagnóstico inmediato de dolencias vasculares, lo que ha propiciado la aparición de dispositivos PPG muy atractivos, fáciles de utilizar y de gran potencial clínico. La morfología del pulso PPG, aparentemente simple, acarrea dificultades en su análisis, dado que no es fácil detectar las inflexiones de la forma de onda. En este sentido la primera y la segunda derivada del pulso PPG permiten un reconocimiento más preciso de los puntos de inflexión, lo que, a su vez, facilita la interpretación de la información contenida en el pulso. Con todo, la segunda derivada del pulso PPG, la señal APG, ha recibido más atención que la primera derivada [Elgendi, 2012b], pues simboliza la aceleración de la sangre en el dedo, en caso de que el dispositivo PPG sea un pulsioxímetro digital. Además, la segunda derivada consigue estabilizar la línea de base, lo que faculta una detección cómoda de las principales características de la señal PPG.

En resumen la fotopletismografía comporta una técnica óptica no invasiva para detectar cambios en el volumen sanguíneo microvascular de los tejidos. Su facilidad de uso, bajo coste y comodidad lo convierten en un área atractiva de investigación en los ámbitos biomédico y clínico, aparte del mercado de consumo de dispositivos PPG [Moraes et al., 2018], con la incursión de la tecnología del Internet de las Cosas (Internet of Things o, en siglas, IoT) y su aplicación a la monitorización inteligente de la salud, lo que se engloba dentro del concepto de Internet de las Cosas de la Salud (Internet of Health Things o, expresado con siglas, IoHT) [da Costa et al., 2018]. No obstante, aún restan flecos por atender debidamente, dado que no siempre es factible obtener buenas y concluyentes mediciones diagnósticas en presencia de artefactos consustanciales a la propia arquitectura tecnológica de adquisición de señales PPG, lo que incidentalmente limita su practicidad a no mucho más allá de un mero diagnóstico preliminar [Sun and Thakor, 2016]. En todo caso se espera que las nuevas tecnologías digitales, más precisas y robustas, vayan corrigiendo los defectos presentes en las señales PPG adquiridas, y, al menos, se disponga de una señal biológica de partida libre de interferencias ajenas a lo que realmente se quiere medir, sin desestimar que aún se desconoce la fuente fisiológica que genera el pulso PPG [Alian and Shelley, 2014], todo un objetivo prioritario e ineludible para expandir el campo de aplicación de esta técnica clínica, perfeccionándose así el conocimiento que puede extraerse de cómo exterioriza un proceso fisiológico comportamientos anómalos y por qué reacciona así y no de otra manera. 


\subsubsection{El estrés}

El ritmo frenético en el que viven inmersas las sociedades desarrolladas está disparando de forma alarmante los casos de ansiedad y depresión entre la población joven y adulta, ${ }^{10}$ somatizándose, cuando estos síntomas persisten en el tiempo, en enfermedades cardiovasculares, esclerosis múltiple, diabetes, la muerte súbita, etcétera [Arza et al., 2018, Yaribeygi et al., 2017, Cohen et al., 2015, McCann et al., 2005]. En definitiva el estado del bienestar se está viendo amenazado por un enemigo en apariencia invisible, pero de efectos demoledores; obedece al nombre de estrés, y, en sus variantes aguda y crónica, está detrás de los episodios de ansiedad y depresión, cada vez más frecuentes en el mundo civilizado [Chudzik et al., 2017]. Por consiguiente, en la época actual, más que nunca, se precisan marcadores biológicos capaces de identificar el nivel de estrés al que se está viendo sometido un individuo, no solo para su detección sino también para prevenir potenciales patologías latentes.

El estrés admite varias definiciones, unas más genéricas y otras más técnicas, si bien todas ellas se refieren a la respuesta biológica del organismo ante una amenaza real o ficticia con objeto de adaptarse a las nuevas circunstancias y así poder sobrevivir. Normalmente el estrés es bien agudo o bien crónico [Arza et al., 2018]. El estrés agudo es pasajero; una vez responde rápidamente al factor estresante, el cuerpo recupera su estado de equilibrio. En cambio el estrés crónico puede durar meses, incluso años; el factor estresante no desaparece y el individuo es incapaz de absorber psicológicamente la sobreexposición, lo que puede provocar con el tiempo serios daños psicológicos y físicos. El mecanismo regulador del estrés reside en el sistema nervioso autónomo; por medio de los sistemas simpático y parasimpático el cuerpo responde de forma colectiva, hormonal, mecánica, física y psicológicamente a la señal de alarma, restaurando en principio la configuración previa tras el episodio de estrés.

Dado que, ante una situación de estrés, el organismo reacciona activando infinidad de marcadores biológicos, no debería resultar muy complicado a priori acceder a algunos de ellos, siempre que la señal adquirida tenga suficiente calidad [Elgendi, 2016]. No obstante, surgen dos limitaciones, una deriva del hecho de que marcadores hormonales del estrés, muy fiables, no se prestan fácilmente a una monitorización continua, y la otra, aún más embarazosa, apunta a que la mayoría de los marcadores extraídos de las señales biológicas, que sí procuran un seguimiento ininterrumpido, no proporcionan una información fiable del grado de estrés presente en el individuo, puesto que las señales biológicas que sirven de base para la caracterización del estrés constituyen respuestas fisiológicas al estrés, esto es, las señales biológicas miden el efecto orgánico inducido del estrés, muchas veces modulado por los rasgos y las circunstancias particulares de cada individuo. Por tanto, una mejor interpretación del estrés demanda un análisis en profundidad de la respuesta dinámica del estrés en relación con el sistema cardiovascular [Javorka et al., 2018, Minakuchi et al., 2013, Schubert et al., 2009, Kyriazis, 2003, Anishchenko et al., 2001, Wagner and Persson, 1998, Lipsitz and Goldberger, 1992], muy en ciernes y abordado de modo intermitente [Sviridova and Sakai, 2015a, Pham et al., 2013, Miao et al., 2010, Tsu-

\footnotetext{
${ }^{10}$ Según prevé la Organización Mundial de la Salud (OMS), en 2030 el trastorno unipolar depresivo será la principal causa de morbilidad del mundo civilizado [Mathers et al., 2008].
} 
da et al., 1992], más que, como viene siendo habitual, un examen cuantitativo de marcadores biológicos [Charlton et al., 2018, Yeo et al., 2017, Lee et al., 2016, Ohno et al., 2015, Elgendi et al., 2015a, Singh et al., 2013]. En este sentido este trabajo sienta las bases teóricas de un próximo estudio sobre cómo se desarrolla el estrés en un individuo desde una perspectiva caótica del pulso PPG, esto es, aplicando métodos derivados del análisis caótico y no lineal de sucesiones temporales, a fin de determinar diferencias cualitativas y cuantitativas en la dinámica del pulso PPG que puedan sugerir marcadores numéricos que diagnostiquen de forma inequívoca cuándo un sujeto está inmerso en una situación de estrés, o, más desafiante todavía, que preludien su inminente aparición y anticipen patologías más graves, de acuerdo con potenciales transiciones de fase en la dinámica cardiovascular [Quiroz-Juárez et al., 2017, Qu et al., 2007, Brouwer et al., 1998]. 



\section{Referencias}

[Abarbanel et al., 1993] Abarbanel, H. D. I., Brown, R., Sidorowich, J. J., and Tsimring, L. S. (1993). The analysis of observed chaotic data in physical systems. Reviews of Modern Physics, 65(4):1331-1392. (páginas 52, 57, 58, 103, 165).

[Akar et al., 2013] Akar, S. A., Kara, S., Latifoğlu, F., and Bilgiç, V. (2013). Spectral analysis of photoplethysmographic signals: The importance of preprocessing. Biomedical Signal Processing and Control, 8(1):16-22. (página 181).

[Alian and Shelley, 2014] Alian, A. A. and Shelley, K. H. (2014). Photoplethysmography. Best Practice 63 Research Clinical Anaesthesiology, 28(4):395-406. (página 183).

[Allen, 2007] Allen, J. (2007). Photoplethysmography and its application in clinical physiological measurement. Physiological Measurement, 28(3):R1-R39. (páginas $178,178,179)$.

[Allen et al., 2005] Allen, J., Oates, C. P., Lees, T. A., and Murray, A. (2005). Photoplethysmography detection of lower limb peripheral arterial occlusive disease: a comparison of pulse timing, amplitude and shape characteristics. Physiological Measurement, 26(5):811-821. (página 178).

[Anishchenko et al., 2001] Anishchenko, T., Igosheva, N., Yakusheva, T., Glushkovskaya-Semyachkina, O., and Khokhlova, O. (2001). Normalized entropy applied to the analysis of interindividual and gender-related differences in the cardiovascular effects of stress. European Journal of Applied Physiology, 85(3-4):287-298. (página 184).

[Arza et al., 2018] Arza, A., Garzón-Rey, J. M., Lázaro, J., Gil, E., López-Antón, R., de la Cámara, C., Laguna, P., Bailón, R., and Aguiló, J. (2018). Measuring acute stress response through physiological signals: towards a quantitative assessment of stress. Medical \& Biological Engineering \& Computing, pages 1-17. (página 184, 184).

[Bachmann et al., 2018] Bachmann, M., Päeske, L., Kalev, K., Aarma, K., Lehtmets, A., Ööpik, P., Lass, J., and Hinrikus, H. (2018). Methods for classifying depression in single channel EEG using linear and nonlinear signal analysis. Computer Methods and Programs in Biomedicine, 155:11-17. (página 179). 
[Beuter et al., 2003] Beuter, A., Glass, L., Mackey, M. C., and Titcombe, M. S. (2003). Nonlinear Dynamics in Physiology and Medicine. Interdisciplinary Applied Mathematics. Springer New York. (página 153).

[Bishop, 2006] Bishop, C. M. (2006). Pattern Recognition and Machine Learning. Information Science and Statistics. Springer. (página 164).

[Blasco and Peris-Lopez, 2018] Blasco, J. and Peris-Lopez, P. (2018). On the feasibility of low-cost wearable sensors for multi-modal biometric verification. Sensors, 18(9):2782. (página 178).

[Brouwer et al., 1998] Brouwer, S. D., Edwards, D. H., and Griffith, T. M. (1998). Simplification of the quasiperiodic route to chaos in agonist-induced vasomotion by iterative circle maps. American Journal of Physiology-Heart and Circulatory Physiology, 274(4):H1315-H1326. (página 185).

[Castaneda et al., 2018] Castaneda, D., Esparza, A., Ghamari, M., Soltanpur, C., and Nazeran, H. (2018). A review on wearable photoplethysmography sensors and their potential future applications in health care. International Journal of Biosensors \& Bioelectronics, 4(4):195-202. (página 179).

[Chan et al., 2013] Chan, E. D., Chan, M. M., and Chan, M. M. (2013). Pulse oximetry: Understanding its basic principles facilitates appreciation of its limitations. Respiratory Medicine, 107(6):789-799. (página 181).

[Charlton et al., 2017] Charlton, P. H., Bonnici, T., Tarassenko, L., Alastruey, J., Clifton, D. A., Beale, R., and Watkinson, P. J. (2017). Extraction of respiratory signals from the electrocardiogram and photoplethysmogram: technical and physiological determinants. Physiological Measurement, 38(5):669-690. (página 180).

[Charlton et al., 2018] Charlton, P. H., Celka, P., Farukh, B., Chowienczyk, P., and Alastruey, J. (2018). Assessing mental stress from the photoplethysmogram: a numerical study. Physiological Measurement, 39(5):054001. (página 185).

[Chudzik et al., 2017] Chudzik, R., Jarosz, K., Gołębiowska, M., and Gołębiowska, B. (2017). Stress disease of the 21st century? Journal of education, Health and Sport, 7(8):20-26. (página 184).

[Cohen et al., 2015] Cohen, B. E., Edmondson, D., and Kronish, I. M. (2015). State of the art review: Depression, stress, anxiety, and cardiovascular disease. American Journal of Hypertension, 28(11):1295-1302. (página 184).

[Crutchfield and Packard, 1982] Crutchfield, J. P. and Packard, N. H. (1982). Symbolic dynamics of one-dimensional maps: Entropies, finite precision, and noise. International Journal of Theoretical Physics, 21(6-7):433-466. (página 177).

[da Costa et al., 2018] da Costa, C. A., Pasluosta, C. F., Eskofier, B., da Silva, D. B., and da Rosa Righi, R. (2018). Internet of health things: Toward intelligent vital signs monitoring in hospital wards. Artificial Intelligence in Medicine, 89:61-69. (página 183). 
[Destexhe et al., 1989] Destexhe, A., Nicolis, G., and Nicolis, C. (1989). Symbolic dynamics from chaotic time series. In NATO ASI Series, volume 208, pages 339343. Springer New York. (página 177).

[Diks et al., 1995] Diks, C., van Houwelingen, J. C., Takens, F., and DeGoede, J. (1995). Reversibility as a criterion for discriminating time series. Physics Letters A, 201(2-3):221-228. (página 174).

[Djawad et al., 2017] Djawad, Y. A., Mu'nisa, A., Rusung, P., Kurniawan, A., Idris, I. S., and Taiyeb, M. (2017). Essential feature extraction of photoplethysmography signal of men and women in their 20s. Engineering Journal, 21(4):259-272. (página 178).

[Eckmann et al., 1987] Eckmann, J.-P., Kamphorst, S. O., and Ruelle, D. (1987). Recurrence plots of dynamical systems. EPL (Europhysics Letters), 4(9):973-977. (página 168).

[Eckmann and Ruelle, 1985] Eckmann, J.-P. and Ruelle, D. (1985). Ergodic theory of chaos and strange attractors. Reviews of Modern Physics, 57(3):617-656. (páginas $36,47,105,106,165,213)$.

[Eerikäinen et al., 2018] Eerikäinen, L. M., Bonomi, A. G., Schipper, F., Dekker, L. R. C., Vullings, R., de Morree, H. M., and Aarts, R. M. (2018). Comparison between electrocardiogram- and photoplethysmogram-derived features for atrial fibrillation detection in free-living conditions. Physiological Measurement, 39(8):084001. (página 178).

[Elgendi, 2012a] Elgendi, M. (2012a). On the analysis of fingertip photoplethysmogram signals. Current Cardiology Reviews, 8(1):14-25. (páginas 178, 179, 180, 182).

[Elgendi, 2012b] Elgendi, M. (2012b). Standard terminologies for photoplethysmogram signals. Current Cardiology Reviews, 8(3):215-219. (página 183).

[Elgendi, 2016] Elgendi, M. (2016). Optimal signal quality index for photoplethysmogram signals. Bioengineering, 3(4):21. (página 184).

[Elgendi et al., 2015a] Elgendi, M., Fletcher, R., Norton, I., Brearley, M., Abbott, D., Lovell, N., and Schuurmans, D. (2015a). On time domain analysis of photoplethysmogram signals for monitoring heat stress. Sensors, 15(10):24716-24734. (página 185).

[Elgendi et al., 2015b] Elgendi, M., Fletcher, R. R., Norton, I., Brearley, M., Abbott, D., Lovell, N. H., and Schuurmans, D. (2015b). Frequency analysis of photoplethysmogram and its derivatives. Computer Methods and Programs in Biomedicine, 122(3):503-512. (página 178).

[Elgendi et al., 2018] Elgendi, M., Liang, Y., and Ward, R. (2018). Toward generating more diagnostic features from photoplethysmogram waveforms. Diseases, 6(1):20. (páginas 177, 178). 
[Gardiner, 2004] Gardiner, C. W. (2004). Handbook of Stochastic Methods for Physics, Chemistry, and the Natural Sciences, 3rd edition. Springer complexity. Springer. (página 162).

[Gil et al., 2010] Gil, E., Orini, M., Bailón, R., Vergara, J. M., Mainardi, L., and Laguna, P. (2010). Photoplethysmography pulse rate variability as a surrogate measurement of heart rate variability during non-stationary conditions. Physiological Measurement, 31(9):1271-1290. (página 183).

[Hardy et al., 1999] Hardy, G. H., Snow, C. P., and de Guzmán, M. (1999). Apología de un matemático. Epistéme (Nivola). Nivola Libros y Ediciones, S. L. (página 154).

[Hertzman, 1937] Hertzman, A. B. (1937). Photoelectric plethysmography of the fingers and toes in man. Experimental Biology and Medicine, 37(3):529-534. (página 179).

[ichiro Komatsu et al., 2003] ichiro Komatsu, K., Fukutake, T., and Hattori, T. (2003). Fingertip photoplethysmography and migraine. Journal of the Neurological Sciences, 216(1):17-21. (página 178).

[Isliker and Kurths, 1993] Isliker, H. and Kurths, J. (1993). A test for stationarity: Finding parts in time series apt for correlation dimension estimates. International Journal of Bifurcation and Chaos, 3(6):1573-1579. (página 168).

[Javorka et al., 2018] Javorka, M., Krohova, J., Czippelova, B., Turianikova, Z., Lazarova, Z., Wiszt, R., and Faes, L. (2018). Towards understanding the complexity of cardiovascular oscillations: Insights from information theory. Computers in Biology and Medicine, 98:48-57. (página 184).

[Jr. et al., 2016] Jr., J. J. A. M., Vieira, M. E. M., Pires, M. B., and Jr., S. L. S. (2016). Sensor fusion and smart sensor in sports and biomedical applications. Sensors, 16(10):1569. (página 178).

[Judd and Mees, 1995] Judd, K. and Mees, A. (1995). On selecting models for nonlinear time series. Physica D: Nonlinear Phenomena, 82(4):426-444. (página 163).

[Kantz, 1994] Kantz, H. (1994). Quantifying the closeness of fractal measures. Physical Review E, 49(6):5091-5097. (páginas 108, 109, 110, 170).

[Kantz and Schreiber, 1995] Kantz, H. and Schreiber, T. (1995). Dimension estimates and physiological data. Chaos: An Interdisciplinary Journal of Nonlinear Science, 5(1):143-154. (páginas 65, 164).

[Kohjitani et al., 2014] Kohjitani, A., Miyata, M., Iwase, Y., Ohno, S., Tohya, A., Manabe, Y., Hashiguchi, T., and Sugiyama, K. (2014). Associations between the autonomic nervous system and the second derivative of the finger photoplethysmogram indices. Journal of Atherosclerosis and Thrombosis, 21(5):501-508. (página 178). 
[Kyriazis, 2003] Kyriazis, M. (2003). Practical applications of chaos theory to the modulation of human ageing: nature prefers chaos to regularity. Biogerontology, 4(2):75-90. (página 184).

[Lee et al., 2016] Lee, D. S., Chong, T. W., and Lee, B. G. (2016). Stress events detection of driver by wearable glove system. IEEE Sensors Journal, 17(1):194204. (página 185).

[Lee et al., 2018] Lee, J., Sohn, J., Park, J., Yang, S., Lee, S., and Kim, H. C. (2018). Novel blood pressure and pulse pressure estimation based on pulse transit time and stroke volume approximation. BioMedical Engineering OnLine, 17(1):1-20. (página 178).

[Liang et al., 2018] Liang, Y., Chen, Z., Liu, G., and Elgendi, M. (2018). A new, short-recorded photoplethysmogram dataset for blood pressure monitoring in china. Scientific Data, 5:180020. (página 180).

[Lipsitz and Goldberger, 1992] Lipsitz, L. A. and Goldberger, A. L. (1992). Loss of 'complexity' and aging. potential applications of fractals and chaos theory to senescence. JAMA, 267(13):1806-1809. (página 184).

[Manuca and Savit, 1996] Manuca, R. and Savit, R. (1996). Stationarity and nonstationarity in time series analysis. Physica D: Nonlinear Phenomena, 99(2-3):134161. (página 170).

[Marmarelis, 2012] Marmarelis, V. (2012). Analysis of Physiological Systems: The White-Noise Approach. Computers in Biology and Medicine. Springer US. (página 164).

[Marwan et al., 2007] Marwan, N., Romano, M. C., Thiel, M., and Kurths, J. (2007). Recurrence plots for the analysis of complex systems. Physics Reports, 438(56):237-329. (página 168).

[Mathers et al., 2008] Mathers, C., Fat, D. M., Boerma, J. T., and Organization, W. H. (2008). The Global Burden of Disease: 2004 Update. Nonserial Publication Series. World Health Organization. (página 184).

[McCann et al., 2005] McCann, U. D., Fauerbach, J. A., and Thombs, B. D. (2005). Anxiety and cardiac disease. Primary Psychiatry, 12(3):47-50. (página 184).

[Miao et al., 2010] Miao, T., Oyama-Higa, M., and Reika, S. (2010). Chaos in plethysmograms during tracking chaos. In 2010 IEEE International Conference on Systems, Man and Cybernetics, pages 3966-3970. IEEE. (página 184).

[Minakuchi et al., 2013] Minakuchi, E., Ohnishi, E., Ohnishi, J., Sakamoto, S., Hori, M., Motomura, M., Hoshino, J., Murakami, K., and Kawaguchi, T. (2013). Evaluation of mental stress by physiological indices derived from finger plethysmography. Journal of Physiological Anthropology, 32(1):17. (página 184). 
[Mischaikow et al., 1999] Mischaikow, K., Mrozek, M., Reiss, J., and Szymczak, A. (1999). Construction of symbolic dynamics from experimental time series. Physical Review Letters, 82(6):1144-1147. (página 177).

[Moço et al., 2018] Moço, A. V., Stuijk, S., and de Haan, G. (2018). New insights into the origin of remote PPG signals in visible light and infrared. Scientific Reports, 8(1). (página 181).

[Moraes et al., 2018] Moraes, J., Rocha, M., Vasconcelos, G., Filho, J. V., de Albuquerque, V., and Alexandria, A. (2018). Advances in photopletysmography signal analysis for biomedical applications. Sensors, 18(6):1894. (páginas 179, 179, 183).

[Nam and Nam, 2017] Nam, Y. and Nam, Y.-C. (2017). Photoplethysmography signal analysis for optimal region-of-interest determination in video imaging on a built-in smartphone under different conditions. Sensors, 17(10):2385. (página 178).

[Nelles, 2013] Nelles, O. (2013). Nonlinear System Identification: From Classical Approaches to Neural Networks and Fuzzy Models. Springer Berlin Heidelberg. (página 162).

[Noah et al., 2018] Noah, B., Keller, M. S., Mosadeghi, S., Stein, L., Johl, S., Delshad, S., Tashjian, V. C., Lew, D., Kwan, J. T., Jusufagic, A., and Spiegel, B. M. R. (2018). Impact of remote patient monitoring on clinical outcomes: an updated meta-analysis of randomized controlled trials. npj Digital Medicine, 1(1):20172. (página 177).

[Ohno et al., 2015] Ohno, S., Miyata, M., Kohjitani, A., Tohya, A., Ohishi, M., and Sugiyama, K. (2015). Associations between blood pressure responses to acute stress and impaired renal function and serum uric acid level. Clinical and Experimental Hypertension, 37(8):656-660. (página 185).

[Palit and Popovic, 2006] Palit, A. K. and Popovic, D. (2006). Computational Intelligence in Time Series Forecasting: Theory and Engineering Applications. Advances in Industrial Control. Springer London. (páginas 162, 164).

[Peart et al., 2018] Peart, D. J., Balsalobre-Fernández, C., and Shaw, M. P. (2018). Use of mobile applications to collect data in sport, health, and exercise science: A narrative review. Journal of Strength and Conditioning Research, page 1. (página 179).

[Pham et al., 2013] Pham, T. D., Thang, T. C., Oyama-Higa, M., and Sugiyama, M. (2013). Mental-disorder detection using chaos and nonlinear dynamical analysis of photoplethysmographic signals. Chaos, Solitons $\&$ Fractals, 51:64-74. (página 184).

[Pomeau and Manneville, 1980] Pomeau, Y. and Manneville, P. (1980). Intermittent transition to turbulence in dissipative dynamical systems. Comm. Math. Phys., 74(2):189-197. (página 166). 
[Provenzale et al., 1992] Provenzale, A., Smith, L. A., Vio, R., and Murante, G. (1992). Distinguishing between low-dimensional dynamics and randomness in measured time series. Physica D: Nonlinear Phenomena, 58(1-4):31-49. (páginas 69, 170).

[Qu et al., 2007] Qu, Z., Shiferaw, Y., and Weiss, J. N. (2007). Nonlinear dynamics of cardiac excitation-contraction coupling: An iterated map study. Physical Review E, 75(1):011927. (página 185).

[Quiroz-Juárez et al., 2017] Quiroz-Juárez, M. A., Vázquez-Medina, R., Ryzhii, E., Ryzhii, M., and Aragón, J. L. (2017). Quasiperiodicity route to chaos in cardiac conduction model. Communications in Nonlinear Science and Numerical Simulation, 42:370-378. (página 185).

[Reguig and Reguig, 2017] Reguig, M. A. B. and Reguig, F. B. (2017). Photoplethysmogram signal processing and analysis in evaluating arterial stiffness. International Journal of Biomedical Engineering and Technology, 23(2/3/4):363-378. (página 178).

[Ridder et al., 2018] Ridder, B. D., Rompaey, B. V., Kampen, J. K., Haine, S., and Dilles, T. (2018). Smartphone apps using photoplethysmography for heart rate monitoring: Meta-analysis. JMIR Cardio, 2(1):e4. (página 178).

[Riley et al., 2012] Riley, M. A., Bonnette, S., Kuznetsov, N., Wallot, S., and Gao, J. (2012). A tutorial introduction to adaptive fractal analysis. Frontiers in Physiology, 3(371):1-10. (página 179).

[Ross, 2007] Ross, S. M. (2007). Introduction to Probability Models, 9th edition. Introduction to Probability Models. Elsevier Science. (página 162).

[Rugh, 1981] Rugh, W. J. (1981). Nonlinear system theory: the Volterra/Wiener approach. Johns Hopkins series in information sciences and systems. Johns Hopkins University Press. (página 164).

[Sato et al., 2013] Sato, S., Miao, T., and Oyama-Higa, M. (2013). Studies on five senses treatment. In Knowledge-Based Systems in Biomedicine and Computational Life Science, pages 155-175. Springer Berlin Heidelberg. (página 179).

[Schäfer and Vagedes, 2013] Schäfer, A. and Vagedes, J. (2013). How accurate is pulse rate variability as an estimate of heart rate variability? International Journal of Cardiology, 166(1):15-29. (páginas 179, 183).

[Schreiber, 1998] Schreiber, T. (1998). Constrained randomization of time series data. Physical Review Letters, 80(10):2105-2108. (página 176).

[Schreiber, 1999] Schreiber, T. (1999). Interdisciplinary application of nonlinear time series methods. Physics Reports, 308(1):1-64. (página 166).

[Schreiber and Schmitz, 1996] Schreiber, T. and Schmitz, A. (1996). Improved surrogate data for nonlinearity tests. Physical Review Letters, 77(4):635-638. (página 176). 
[Schreiber and Schmitz, 1997] Schreiber, T. and Schmitz, A. (1997). Discrimination power of measures for nonlinearity in a time series. Physical Review E, 55(5):54435447. (páginas 170, 174).

[Schrödinger, 1984] Schrödinger, E. (1984). ¿Qué es la vida? Cuadernos ínfimos; 107/Serie Metatemas. Tusquets Editores. (página 154).

[Schubert et al., 2009] Schubert, C., Lambertz, M., Nelesen, R., Bardwell, W., Choi, J.-B., and Dimsdale, J. (2009). Effects of stress on heart rate complexity - a comparison between short-term and chronic stress. Biological Psychology, 80(3):325-332. (página 184).

[Semmlow and Griffel, 2014] Semmlow, J. L. and Griffel, B. (2014). Biosignal and Medical Image Processing, 3rd edition. CRC Press. (página 155).

[Sen and McGill, 2017] Sen, J. and McGill, D. (2017). Fractal analysis of heart rate variability as a predictor of mortality: A systematic review. Heart, Lung and Circulation, 26:S165-S166. (página 179).

[Shelhamer, 2007] Shelhamer, M. (2007). Nonlinear Dynamics in Physiology: A State-space Approach. World Scientific. (páginas 161, 204).

[Singh et al., 2013] Singh, R. R., Conjeti, S., and Banerjee, R. (2013). Assessment of driver stress from physiological signals collected under real-time semi-urban driving scenarios. International Journal of Computational Intelligence Systems, 7(5):909-923. (página 185).

[Sociedad Española de Cardiología, 2016] Sociedad Española de Cardiología (2016). Mortalidad cardiovascular. la enfermedad cardiovascular primera causa de muerte. Last checked on September, 2018. (página 178).

[Stefanovska, 1999] Stefanovska, A. (1999). Physics of the human cardiovascular system. Contemporary Physics, 40(1):31-55. (página 179).

[Stewart, 1989] Stewart, I. (1989). Portraits of chaos. New Scientist, 124:42-47. (página 168).

[Sun and Thakor, 2016] Sun, Y. and Thakor, N. (2016). Photoplethysmography revisited: From contact to noncontact, from point to imaging. IEEE Transactions on Biomedical Engineering, 63(3):463-477. (página 183).

[Sviridova, 2015] Sviridova, N. (2015). Study on chaotic behavior of human photoplethysmogram by comprehensive nonlinear time series analysis. $\mathrm{PhD}$ thesis, Tokyo University of Agriculture and Technology. (páginas 179, 180, 206).

[Sviridova and Sakai, 2015a] Sviridova, N. and Sakai, K. (2015a). Application of photoplethysmogram for detecting physiological effects of tractor noise. Engineering in Agriculture, Environment and Food, 8(4):313-317. (páginas 179, 184). 
[Sviridova and Sakai, 2015b] Sviridova, N. and Sakai, K. (2015b). Human photoplethysmogram: new insight into chaotic characteristics. Chaos, Solitons 63 Fractals, 77:53-63. (página 179).

[Tamura et al., 2014] Tamura, T., Maeda, Y., Sekine, M., and Yoshida, M. (2014). Wearable photoplethysmographic sensors - past and present. Electronics, 3(2):282-302. (página 178).

[Theiler et al., 1992] Theiler, J., Eubank, S., Longtin, A., Galdrikian, B., and Farmer, J. D. (1992). Testing for nonlinearity in time series: the method of surrogate data. Physica D: Nonlinear Phenomena, 58(1-4):77-94. (páginas 70, 172).

[Theiler and Prichard, 1996] Theiler, J. and Prichard, D. (1996). Constrainedrealization monte-carlo method for hypothesis testing. Physica D: Nonlinear Phenomena, 94(4):221-235. (página 174).

[Theiler and Prichard, 1997] Theiler, J. and Prichard, D. (1997). Using surrogate surrogate data to calibrate the actual rate of false positives in tests for nonlinearity in time series. Fields Institute Communications, 11:99-113. (página 172).

[Tsuda et al., 1992] Tsuda, I., Tahara, T., and Iwanaga, H. (1992). Chaotic pulsation in human capillary vessels and its dependence on mental and physical conditions. International Journal of Bifurcation and Chaos, 2(2):313-324. (página 184).

[Vidal, 1993] Vidal, R. V. V. (1993). Applied Simulated Annealing. Springer Berlin Heidelberg. (página 177).

[Volterra, 1959] Volterra, V. (1959). Theory of Functionals of Integral and Integrodifferential Equations. Dover books on mathematics. Dover Publications. (página 164).

[Wagner and Persson, 1998] Wagner, C. D. and Persson, P. B. (1998). Chaos in the cardiovascular system: an update. Cardiovascular Research, 40(2):257-264. (página 184).

[Webster, 1997] Webster, J. G. (1997). Design of Pulse Oximeters. Series in Medical Physics and Biomedical Engineering. CRC Press. (página 181).

[Wiener, 1966] Wiener, N. (1966). Nonlinear Problems in Random Theory. MIT Press Classics. MIT Press. (página 164).

[Wiggins, 2003] Wiggins, S. (2003). Introduction to Applied Nonlinear Dynamical Systems and Chaos, 2nd edition. Texts in Applied Mathematics. Springer New York. (página 167).

[Witt et al., 1998] Witt, A., Kurths, J., and Pikovsky, A. (1998). Testing stationarity in time series. Physical Review E, 58(2):1800-1810. (página 168).

[Wu et al., 2017] Wu, H.-T., Lin, G.-M., Haryadi, B., Yang, C.-M., and Chu, H.-C. (2017). Glycemic control, hand activity, and complexity of biological signals in diabetes mellitus. Complexity, 2017:1-9. (página 178). 
[Yaribeygi et al., 2017] Yaribeygi, H., Panahi, Y., Sahraei, H., Johnston, T. P., and Sahebkar, A. (2017). The impact of stress on body function: a review. EXCLI Journal; 16:Doc1057; ISSN 1611-2156, 16:1057-1072. (página 184).

[Yeo et al., 2017] Yeo, M., Lim, S., and Yoon, G. (2017). Analysis of biosignals during immersion in computer games. Journal of Medical Systems, 42(1):1-9. (página 185). 


\section{Capítulo 6}

\section{Resultados obtenidos}

El presente capítulo aglutina los resultados alcanzados cuando las diferentes señales PPG, en consonancia con lo emplazado en el capítulo 5, son sometidas a un análisis no lineal, conforme a la teoría caótica de los sistemas dinámicos, cuyas líneas maestras se introdujeron en los capítulos 2, 3 y 4 . El propósito no es otro que intuir la naturaleza dinámica de este tipo particular de señales biológicas, en contraste con otras señales de referencia cuyo comportamiento es conocido a priori (véase el apéndice A para una descripción más completa), sentándose las bases, para una investigación futura, de cómo puede evolucionar su comportamiento funcional, si fuere el caso, y debido a qué causas fisiológicas, cuando los individuos de los que se han adquirido las señales PPG se exponen a situaciones estresantes.

Tabla 6.1: Momentos estadísticos de las señales PPG.

\begin{tabular}{|ccccccccc|}
\hline \hline señal PPG & media & mediana & moda & $\begin{array}{c}\text { desviación } \\
\text { típica }\end{array}$ & $\begin{array}{c}\text { coeficiente } \\
\text { variación }\end{array}$ & $\begin{array}{c}\text { coeficiente } \\
\text { asimetría }\end{array}$ & curtosis \\
\hline individuo núm. 1 (PPG1) & 0,3458 & 0,2733 & 0,1669 & 0,2149 & $62,1583 \%$ & $-7,98102 \cdot 10^{-36}$ & $3,93346 \cdot 10^{-46}$ \\
\hline individuo núm. 2 (PPG2) & 0,4460 & 0,3895 & 0,3171 & 0,1566 & $35,1171 \%$ & $-6,29473 \cdot 10^{-39}$ & $2,86638 \cdot 10^{-50}$ \\
\hline individuo núm. 3 (PPG3) & 0,3384 & 0,2885 & 0,1043 & 0,2103 & $62,1481 \%$ & $7,47158 \cdot 10^{-35}$ & $7,76093 \cdot 10^{-45}$ \\
\hline individuo núm. 4 (PPG4) & 0,3198 & 0,2901 & 0,0701 & 0,2204 & $68,9153 \%$ & $9,22528 \cdot 10^{-36}$ & $4,77166 \cdot 10^{-46}$ \\
\hline individuo núm. 5 (PPG5) & 0,5244 & 0,4940 & 0,3792 & 0,1660 & $31,6529 \%$ & $3,03541 \cdot 10^{-36}$ & $1,08390 \cdot 10^{-46}$ \\
\hline \hline
\end{tabular}

Todas las sucesiones temporales constan de 15.000 puntos y han sido normalizadas, de forma que su rango dinámico comprenda el intervalo $[0,1]$. En el caso de las señales biológicas, referidas a señales PPG capturadas, con la ayuda de un oxímetro de pulso, de cinco individuos elegidos aleatoriamente, adicionalmente fueron filtradas mediante un filtro de Butterworth, configurado como filtro paso banda con frecuencias de corte inferior y superior de $0,2 \mathrm{~Hz}$ y $8 \mathrm{~Hz}$, respectivamente, a fin de reducir posibles artefactos debidos a movimientos involuntarios de la mano durante la sesión de adquisición de los datos. Los momentos de menor orden se ilustran en la tabla 6.1. Cabe destacar que se han incluido tres parámetros que, desde el ámbito de la estadística descriptiva, caracterizan la distribución de los datos: el coeficiente de variación (en \%) para medir el porcentaje de dispersión de los datos, el coeficiente de asimetría, también denominado Pearson's coefficient of skewness, para medir el gra- 
do de simetría de la distribución alrededor de la media, y la curtosis (Kurtosis), que identifica cómo de puntiaguda es la distribución de frecuencias; a mayor curtosis más puntiaguda es la distribución. Con una curtosis nula se dispone de una distribución normal.

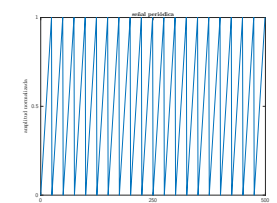

(a)

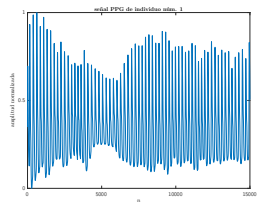

(f)

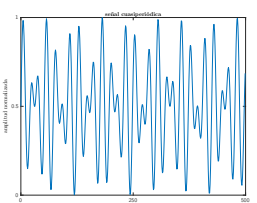

(b)

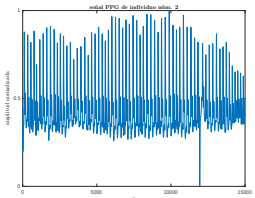

$(\mathrm{g})$

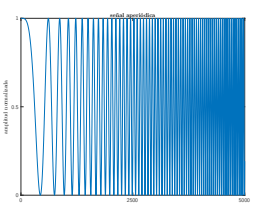

(c)

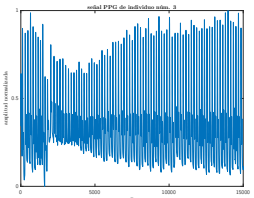

(h)

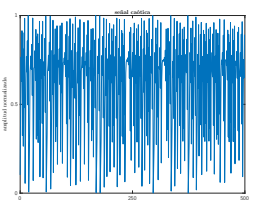

(d)

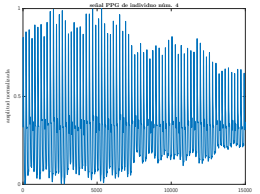

(i)

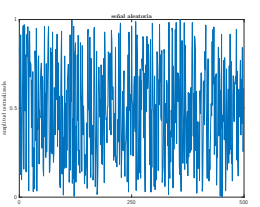

(e)

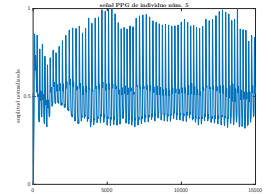

$(\mathbf{j})$

Figura 6.1: Evolución temporal de las señales de referencia (a)-(e) y de las señales PPG (f)-(j), adquiridas de cinco individuos elegidos aleatoriamente.

La representación temporal de las señales se muestra en la figura 6.1, si bien por razones de claridad, de manera que pueda intuirse visualmente la forma de onda de su evolución, no se representan gráficamente, en todos los casos, los 15.000 puntos inicialmente disponibles, limitándose la muestra visual a 500 o a 5.000 integrantes.

\subsection{Reconstrucción del espacio de estados}

La reconstrucción del espacio de estados, de acuerdo con lo dispuesto en el capítulo 3, comporta un paso preliminar indispensable en el análisis no lineal de todo sistema dinámico del que únicamente se dispone, como es habitual, de un observable obtenido de forma experimental, a saber, de una sucesión temporal escalar. De la congruente reconstrucción del espacio de estados, ante la inexistencia del sistema de ecuaciones que determinan la dinámica del proceso físico bajo estudio y en presencia, invariablemente, de ruido, depende que todo análisis posterior sea mayormente concluyente y obedezca a criterios objetivos y coherentes, firmemente asentados, al margen de que pueda o no establecerse, sobre la base de un posible modelo de comportamiento predictivo que resuelva en cierta medida la interacción lineal o no lineal entre las variables, una correspondencia directa entre las variables dinámicas que conforman la propia dinámica del sistema sugerida y su significación física.

Por tanto, y con miras a la reconstrucción del espacio de estados, se procede a su determinación detallada, para la señal PPG de un individuo cualquiera, si bien en la tabla 6.2 se listan, aplicándose el mismo procedimiento en todos los casos, los resultados adquiridos tanto para las señales de referencia como para las cinco señales PPG, concernientes a cinco individuos escogidos al azar; como ejemplo ilustrativo se propone el análisis de la señal PPG del individuo número 4, dado que un reconocimiento preliminar de su evolución temporal exhibe en cierta medida una mayor 
estacionariedad (menor variabilidad) en los ciclos PPG.

\subsubsection{Selección del parámetro $\tau$}

Para la determinación del parámetro $\tau$ se ha optado por dos métodos: la función de autocorrelación y la información mutua. En el primer caso se selecciona como valor de $\tau$ el primer paso por cero de la función de autocorrelación, como así se indica en la figura 6.2.

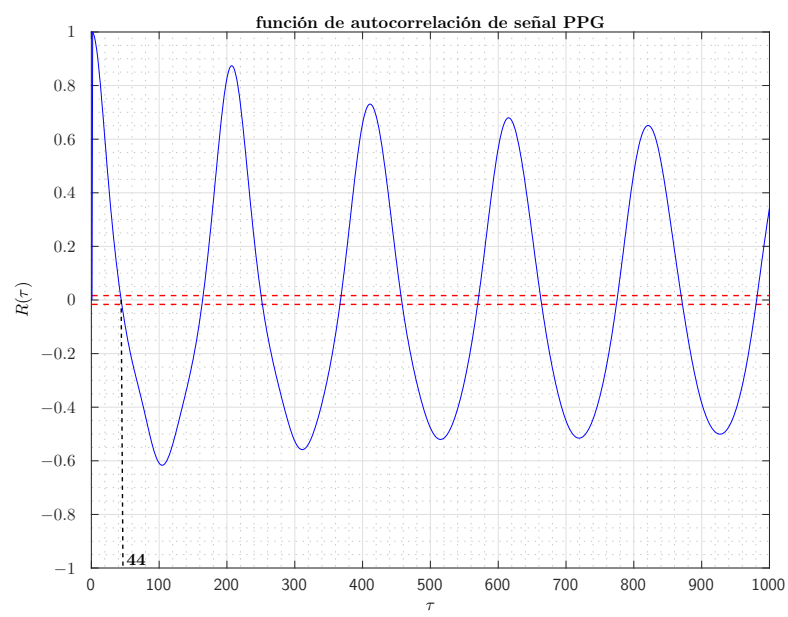

Figura 6.2: Función de autocorrelación de la señal PPG para el individuo número 4 , en la que se señala el primer paso por cero, cuando $\tau=44$.

En relación con la información mutua se selecciona como valor de $\tau$ el primer mínimo de la información mutua, como así se ilustra en la figura 6.3.

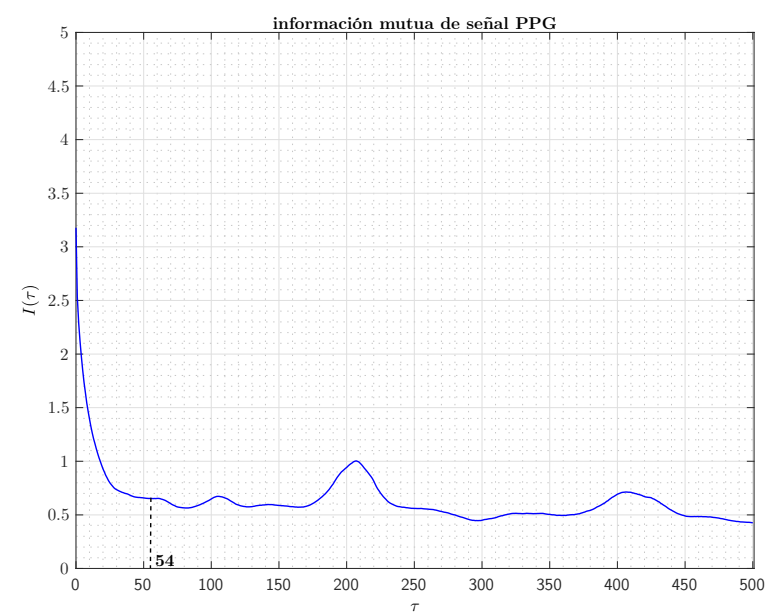

Figura 6.3: Información mutua de la señal PPG para el individuo número 4, en la que se indica el primer mínimo, cuando $\tau=54$. 


\subsubsection{Selección del parámetro $m$}

Para la determinación del parámetro $m$ se ha optado por tres métodos: el análisis de componentes principales, el método de los falsos vecinos más cercanos y la dimensión de correlación.

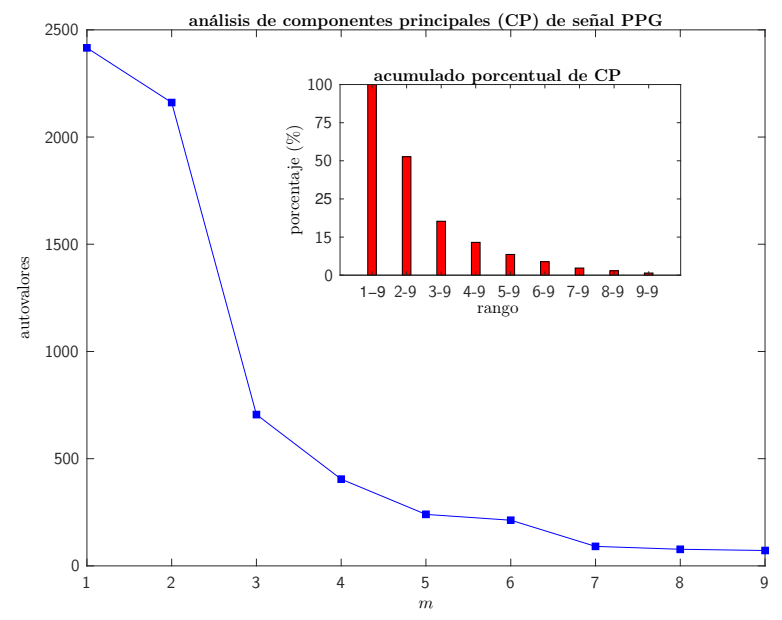

Figura 6.4: Análisis de componentes principales de la señal PPG para el individuo número 4; en el recuadro interior no se aprecia claramente con cuántas direcciones o dimensiones puede caracterizarse el comportamiento dinámico del atractor.

De acuerdo con el análisis de componentes principales (véase la figura 6.4), se obtiene una dimensión de reconstrucción $m$ del orden de 4 o 5 , pues a partir de $m \geqslant 6$ las componentes concentran menos del $15 \%$ de la varianza de los datos. En este sentido ha de recurrirse a criterios subjetivos, pues que un $15 \%$ de la varianza sea despreciable no es obvio y requiere un análisis más pormenorizado de los datos, así como del ruido contenido en los mismos.

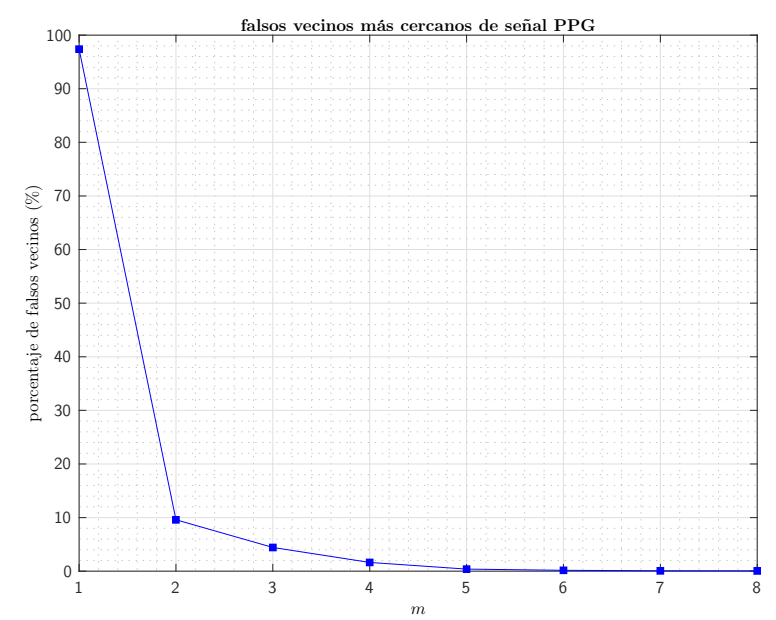

Figura 6.5: Falsos vecinos más cercanos de la señal PPG para el individuo número 4. A partir de $m=4$ el número de falsos vecinos más cercanos se reduce prácticamente a cero. 
Referente a los falsos vecinos más cercanos se juzga pertinente una dimensión de reconstrucción $m=4$, si bien no es hasta $m=6$ cuando, según la figura 6.5, se alcanza un porcentaje numéricamente nulo de falsos vecinos; es probable que la longitud finita de los datos y la presencia de ruido estén contribuyendo en alguna medida en la manifestación de falsos vecinos con $m=4$.

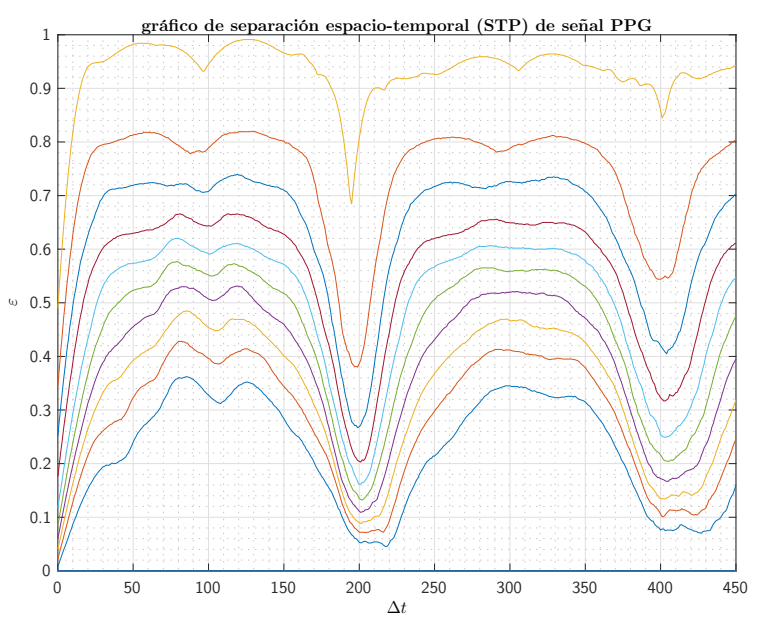

Figura 6.6: Gráfico de separación espacio-temporal de la señal PPG para el individuo número 4 . Como puede advertirse, en torno a $\Delta t \approx 300$ las líneas de contorno mantienen valores aproximadamente constantes.

Finalmente, con la dimensión de correlación $D_{2}$ puede obtenerse un valor, adicionando cierta holgura, dicho sea de paso, conservadora, de $m \geqslant 2 \cdot\left\lceil D_{2}\right\rceil+1$, si bien es posible que con $m \geqslant 2 \cdot\left\lceil D_{2}\right\rceil$, o incluso con $m \geqslant\left\lceil D_{2}\right\rceil$, pudieran conseguirse resultados igualmente fiables.

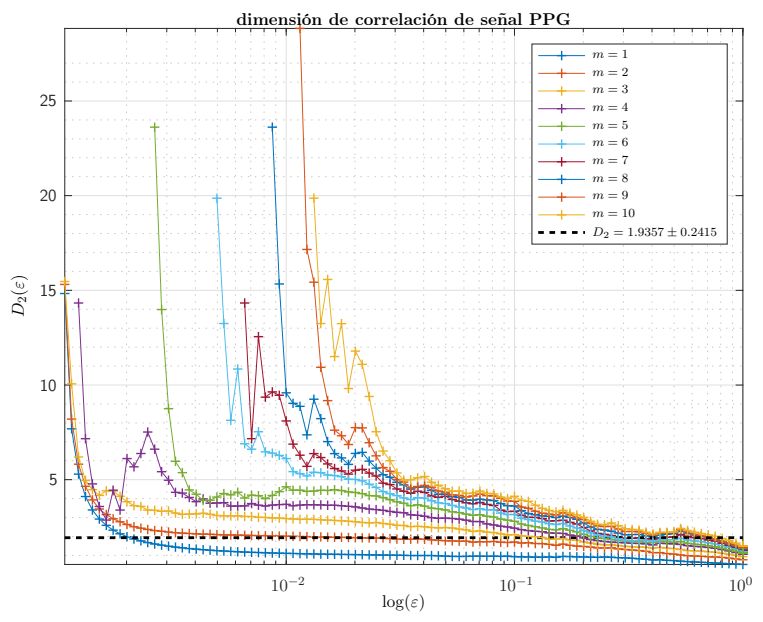

Figura 6.7: Dimensión de correlación de la señal PPG para el individuo número 4. A partir de $m=3$ la dimensión de correlación se confina a un valor constante en un rango intermedio y reducido de escalas $\varepsilon$.

A fin de suprimir factores espurios en la estimación de la dimensión de correlación, 
conforme al gráfico de separación espacio-temporal (remítase a la figura 6.6), se opta por considerar una ventana de Theiler de 300 muestras. Atendiendo a la figura 6.7, se observa cómo las curvas de dimensión se saturan en la proximidad de una línea constante de altura $D_{2} \approx 1,9357$; de esta forma, la dimensión de reconstrucción adquirida asciende a $m \geqslant 5$.

Tabla 6.2: Parámetros $\tau$ y $m$ de las señales de referencia y de las señales PPG, adquiridas de cinco individuos elegidos aleatoriamente.

\begin{tabular}{|lcc|}
\hline \multicolumn{1}{|c}{ señal evaluada } & $\tau$ & $m$ \\
\hline señal periódica & 1 & 2 \\
\hline señal cuasiperiódica & 5 & 5 \\
\hline señal aperiódica & 6 & 5 \\
\hline señal caótica & 20 & 2 \\
\hline señal aleatoria & 2 & 2 \\
\hline individuo núm. 1 (PPG1) & 81 & 5 \\
\hline individuo núm. 2 (PPG2) & 35 & 5 \\
\hline individuo núm. 3 (PPG3) & 30 & 5 \\
\hline individuo núm. 4 (PPG4) & 54 & 5 \\
\hline individuo núm. 5 (PPG5) & 33 & 5 \\
\hline \hline
\end{tabular}

En la tabla 6.2 se muestran los parámetros $\tau$ y $m$, una vez aplicada la metodología previa de reconstrucción del espacio de estados, de todas las señales de referencia y de las cinco señales PPG, correspondientes a cinco individuos elegidos al azar, que servirán de parámetros de partida para la obtención de resultados en secciones posteriores. En todo caso cabe destacar que de todas las opciones estudiadas son la información mutua, para el caso del parámetro $\tau$, y los falsos vecinos más cercanos, en lo referido al parámetro $m$, los que han brindado valores más consecuentes, porque, por un lado, el primer mínimo de la información mutua reduce las correlaciones lineales y no lineales entre las componentes de cada vector de estado reconstruido, y, por otro lado, el método de los falsos vecinos más cercanos, al recurrir a aspectos topológicos del espacio de estados reconstruido, aplaca el efecto del ruido en el cómputo algorítmico, no así, por el contrario, tanto en el análisis de componentes principales, donde no resulta tan definitiva la distribución de la varianza de los datos entre las diferentes direcciones, como en la dimensión de correlación, en la que, con el gravamen adicional propiciado por sucesiones temporales de longitud finita, no es tan manifiesta una saturación de las curvas en escalas intermedias. Por lo tanto, la tabla 6.2 refleja los valores de la información mutua y de los falsos vecinos más cercanos, en términos de $\tau$ y $m$, respectivamente. 


\subsection{Grado de complejidad}

La evaluación del grado de complejidad de una señal autoriza la posibilidad de comparar diferentes sucesiones temporales y distinguir si su comportamiento es regular, caótico o aleatorio, esto es, discernir toda una gama de dinámicas alternativas a los casos más extremos, bien regular o periódico o bien completamente aleatorio, lo que, en el caso de las señales biológicas, podría ayudar a diagnosticar enfermedades o disfunciones orgánicas. Las medidas de la complejidad que se abordan en este trabajo atienden a la entropía, la dimensión fractal y los exponentes de Lyapunov, como se desarrolló en el marco teórico de la sección 4.2.

\subsubsection{Exponentes de Lyapunov}

Un ingrediente de la complejidad dinámica de un sistema físico deriva del espectro de exponentes de Lyapunov (véase la subsección 4.2.1 para más detalles), si bien es su mayor exponente $\lambda_{\text {máx }}$ el que determina el grado de sensibilidad de la evolución dinámica del sistema a las condiciones iniciales y, por tanto, del grado de predictibilidad de estados futuros.

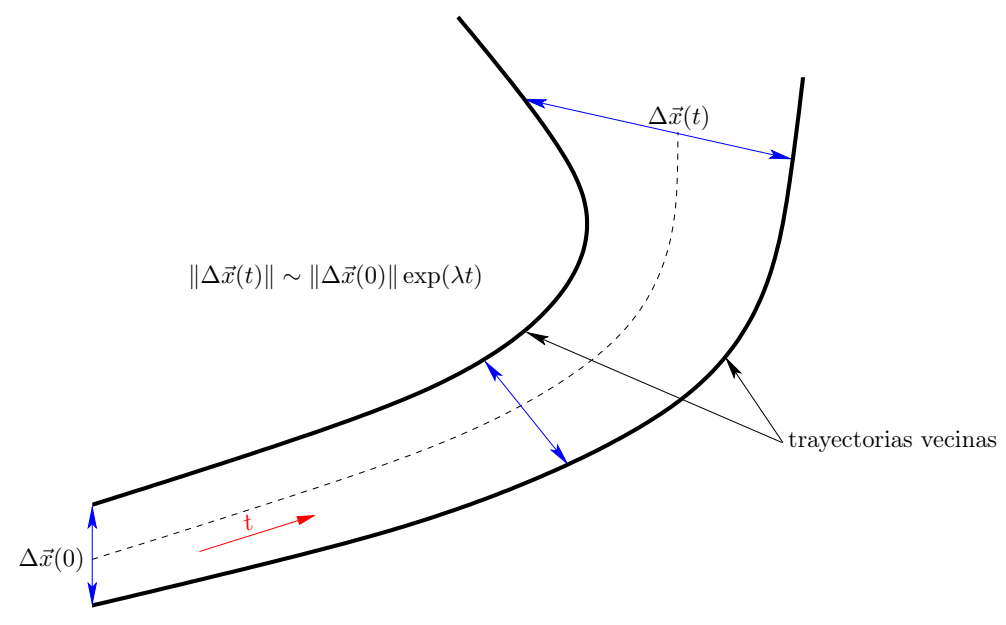

Figura 6.8: Divergencia exponencial de trayectorias inicialmente cercanas entre sí, indicativo de la presencia de un exponente de Lyapunov positivo, y, por tanto, de caos determinista.

Un valor positivo de $\lambda_{\text {máx }}$, como ilustra la figura 6.8 , es indicativo de sistemas complejos, en general, y de una dinámica caótica, en particular.

- Un $\lambda_{\text {máx }}<0$ es característico de un sistema disipativo. En el límite asintótico el sistema se muestra estable, tanto más estable cuanto más negativo sea el valor de $\lambda_{\text {máx }}$.

- Un $\lambda_{\text {máx }}=0$ se traduce en un punto fijo neutral y describe un sistema en un estado de equilibrio, o cerca de experimentar una transición al caos cuando $\lambda_{\text {máx }} \approx 0$. 
- Un $\lambda_{\text {máx }}>0$ caracteriza una órbita inestable y caótica. Estados próximos, a escala incluso infinitesimal, pronto se separan de manera notoria; cuanto más

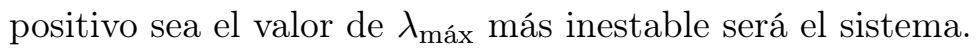

Tabla 6.3: Mayor exponente de Lyapunov $\lambda_{\operatorname{máx}}$ con contraste de hipótesis para un nivel de significación del $5 \%$. La hipótesis nula $\mathbf{H}_{0}: \lambda_{\text {máx }} \geqslant 0$ indica la presencia de un comportamiento caótico. La hipótesis alternativa $\mathbf{H}_{1}: \lambda_{\text {máx }}<0$ sugiere una dinámica no caótica. Si $\mathbf{H}=0$ no se rechaza la hipótesis nula; en caso contrario, si $\mathbf{H}=1$ se rechaza la hipótesis nula. En todos los casos se identifica el intervalo de confianza (IC). Presumiblemente, un intervalo de confianza infinito es consecuencia del tamaño limitado de la sucesión temporal, al igual que también podría derivarse de la dispersión de los datos; a mayor dispersión de los datos la conclusión es menos cierta y el intervalo de confianza es mayor [du Prel et al., 2009].

\begin{tabular}{|lcccc|}
\hline \multicolumn{1}{|c}{ señal evaluada } & H & valor de $p$ & $\lambda_{\text {máx }}$ & valor de IC \\
\hline señal periódica & 1 & 0 & $-0,0655$ & {$[-0,0655 \infty[$} \\
\hline señal cuasiperiódica & 0 & 0,4753 & $-6,6366 \cdot 10^{-4}$ & {$[-0,0183 \infty[$} \\
\hline señal aperiódica & 1 & $4,6925 \cdot 10^{-11}$ & $-0,5913$ & {$[-0,7415 \infty[$} \\
\hline señal caótica & 0 & 1 & 0,8428 & {$[+0,7312 \infty[$} \\
\hline señal aleatoria & 1 & $9,3982 \cdot 10^{-57}$ & $-0,4770$ & {$[-0,5266 \infty[$} \\
\hline individuo núm. 1 (PPG1) & 1 & $1,1005 \cdot 10^{-5}$ & $-0,0254$ & {$[-0,0353 \infty[$} \\
\hline individuo núm. 2 (PPG2) & 1 & 0 & $-0,0488$ & {$[-0,0501 \infty[$} \\
\hline individuo núm. 3 (PPG3) & 1 & $1,1075 \cdot 10^{-72}$ & $-0,0290$ & {$[-0,0316 \infty[$} \\
\hline individuo núm. 4 (PPG4) & 0 & 0,4878 & $-2,3357 \cdot 10^{-4}$ & {$[-0,0128 \infty[$} \\
\hline individuo núm. 5 (PPG5) & 1 & 0 & $-0,0419$ & {$[-0,0432 \infty[$} \\
\hline \hline
\end{tabular}

Las tablas 6.3 y 6.4 pormenorizan el máximo exponente de Lyapunov de todas las señales bajo estudio, conforme a un test estadístico con contraste de hipótesis [BenSaïda, 2015], y el espectro de exponentes de Lyapunov, de acuerdo con el planteamiento original de M. Sano et al. [Sano and Sawada, 1985], respectivamente. A tenor de los valores numéricos de las tablas 6.3 y 6.4, las señales PPG no acusan una marcada tendencia hacia valores bien negativos o bien positivos, ${ }^{1}$ si bien en cualquier caso sí guardan una estrecha relación con los valores exhibidos por la señal cuasiperiódica. Que los valores de los exponentes de Lyapunov se sitúen en una franja muy estrecha alrededor de cero puede ser indicativo de la presencia de una transición al caos (véase la subsección 4.1.2), en el caso de los valores positivos

\footnotetext{
${ }^{1}$ Las limitaciones de los métodos de cómputo de los exponentes de Lyapunov, sumado al ruido presente en los datos, provocan que en algunos casos se hayan evidenciado valores positivos en el máximo exponente de Lyapunov incluso en sistemas no caóticos [Shelhamer, 2007].
} 
del máximo exponente de Lyapunov, o sintomático, en el caso de valores negativos, de una transición aperiódica hacia atractores extraños no caóticos, llamados SNA (Strange Nonchaotic Attractors) por sus siglas en inglés [Grebogi et al., 1984], atendiendo a sistemas forzados cuasiperiódicamente, como podría suceder con los mapas de la circunferencia, lo que además, por otro lado, explicaría la naturaleza fractal de estas respuestas fisiológicas.

Tabla 6.4: Espectro de exponentes de Lyapunov, conforme al método de M. Sano et al., así como la dimensión de Lyapunov, o dimensión de Kaplan-Yorke $D_{\mathrm{KY}}$, y la entropía de Kolmogórov-Sinái $h_{\mathrm{KS}}$, de acuerdo con los postulados de una aproximación preliminar de la identidad de Pesin.

\begin{tabular}{|c|c|c|c|c|}
\hline señal evaluada & $m$ & espectro de $\lambda_{i}$ & $D_{\mathbf{K Y}}$ & $h_{\mathrm{KS}}$ \\
\hline señal periódica & 2 & $\begin{array}{l}\lambda_{1}=0 \\
\lambda_{2}=0\end{array}$ & - & 0,0000 \\
\hline señal cuasiperiódica & 5 & $\begin{array}{l}\lambda_{1}=+8,3773 \cdot 10^{-2} \\
\lambda_{2}=+1,2758 \cdot 10^{-3} \\
\lambda_{3}=-2,1249 \cdot 10^{-3} \\
\lambda_{4}=-1,8353 \cdot 10^{-1} \\
\lambda_{5}=-3,7184 \cdot 10^{-1}\end{array}$ & 3,4510 & 0,0850 \\
\hline señal aperiódica & 5 & $\begin{array}{l}\lambda_{1}=+1,6968 \cdot 10^{-1} \\
\lambda_{2}=+5,4748 \cdot 10^{-3} \\
\lambda_{3}=-7,4616 \cdot 10^{-3} \\
\lambda_{4}=-1,3011 \cdot 10^{-1} \\
\lambda_{5}=-5,0050 \cdot 10^{-1}\end{array}$ & 4,0751 & 0,1752 \\
\hline señal caótica & 2 & $\begin{array}{l}\lambda_{1}=+4,2355 \cdot 10^{-1} \\
\lambda_{2}=-1,5731\end{array}$ & 1,2692 & 0,4235 \\
\hline señal aleatoria & 2 & $\begin{array}{l}\lambda_{1}=+1,0548 \\
\lambda_{2}=-2,4707 \cdot 10^{-1}\end{array}$ & 2,0000 & 1,0548 \\
\hline individuo núm. 1 (PPG1) & 5 & $\begin{array}{l}\lambda_{1}=+5,0695 \cdot 10^{-2} \\
\lambda_{2}=-3,1828 \cdot 10^{-3} \\
\lambda_{3}=-4,9225 \cdot 10^{-2} \\
\lambda_{4}=-2,5680 \cdot 10^{-1} \\
\lambda_{5}=-6,0157 \cdot 10^{-1}\end{array}$ & 2,9652 & 0,0507 \\
\hline individuo núm. 2 (PPG2) & 5 & $\begin{array}{l}\lambda_{1}=+5,6656 \cdot 10^{-2} \\
\lambda_{2}=+1,3506 \cdot 10^{-2} \\
\lambda_{3}=-5,0636 \cdot 10^{-2} \\
\lambda_{4}=-1,9242 \cdot 10^{-1} \\
\lambda_{5}=-6,5947 \cdot 10^{-1}\end{array}$ & 3,1015 & 0,0702 \\
\hline
\end{tabular}


Tabla 6.4: Espectro de exponentes de Lyapunov, conforme al método de M. Sano et $a l$, así como la dimensión de Lyapunov, o dimensión de Kaplan-Yorke $D_{\mathrm{KY}}$, y la entropía de Kolmogórov-Sinái $h_{\mathrm{KS}}$, de acuerdo con los postulados de una aproximación preliminar de la identidad de Pesin (cont.).

\begin{tabular}{|ccccc|}
\hline señal evaluada & $m$ & espectro de $\lambda_{i}$ & $D_{\mathbf{K Y}}$ & $h_{\mathbf{K S}}$ \\
\hline & & $\lambda_{1}=+5,8791 \cdot 10^{-2}$ & & \\
individuo núm. 3 (PPG3) & $\begin{array}{l}\lambda_{2}=+1,4805 \cdot 10^{-2} \\
\lambda_{3}=-4,7613 \cdot 10^{-2}\end{array}$ & 3,1270 & 0,0736 \\
& $\lambda_{4}=-2,0457 \cdot 10^{-1}$ & & \\
& $\lambda_{5}=-7,7598 \cdot 10^{-1}$ & & \\
& & & \\
& $\lambda_{1}=+4,8745 \cdot 10^{-2}$ & & \\
& $\lambda_{2}=+8,8125 \cdot 10^{-3}$ & & \\
individuo núm. 4 (PPG4) & $\lambda_{3}=-3,1214 \cdot 10^{-2}$ & 3,1469 & 0,0576 \\
& $\lambda_{4}=-1,7936 \cdot 10^{-1}$ & & \\
& $\lambda_{5}=-6,5474 \cdot 10^{-1}$ & & \\
\hline & & & \\
& $\lambda_{1}=+5,3972 \cdot 10^{-2}$ & & \\
individuo núm. 5 (PPG5) & $\lambda_{2}=+8,3098 \cdot 10^{-3}$ & & \\
& $\lambda_{3}=-4,8679 \cdot 10^{-2}$ & 3,0725 & 0,0623 \\
& $\lambda_{4}=-1,8751 \cdot 10^{-1}$ & & \\
& $\lambda_{5}=-6,9342 \cdot 10^{-1}$ & & \\
\hline \hline
\end{tabular}

La tendencia, según la tabla 6.4, hacia valores positivos del exponente máximo de Lyapunov avalaría las conclusiones de [Sviridova, 2015], por las que las señales PPG son caóticas; no obstante, los valores negativos de la tabla 6.3 revelan otra posible dinámica, no caótica, asociada en cierta medida con las respuestas aperiódicas que muestran ciertos sistemas forzados cuasiperiódicamente; son, como ya se adelantó con anterioridad, los llamados atractores extraños no caóticos, con múltiples aplicaciones en materias tan dispares e interesantes como la implementación de puertas lógicas, aprovechando la no linealidad y el forzado cuasiperiódico del sistema [Aravindh et al., 2018], o el acoplamiento y la sincronización no lineal entre subsistemas de por sí complejos, como ha quedado demostrado con el mecanismo de funcionamiento subyacente en un electroencefalograma (EEG) [Prasad et al., 2001].

\subsubsection{Dimensiones}

El denominador común de la extensa taxonomía que congrega la noción de dimensión apunta al escalamiento que experimenta una magnitud física contenida en un elemento infinitesimal de volumen, véanse su volumen, su masa, la información, etc., con su tamaño, o al número de grados de libertad presentes en un sistema físico. En cualquier caso la dimensión comprende una medida del número de variables que describen una dinámica física y cómo evolucionan sus valores en una escala temporal y/o espacial. En este apartado se examinan la dimensión fractal, en términos de llenado del espacio a medida que progresa temporalmente la dinámica consus- 
tancial a una sucesión temporal, y las dimensiones de información y de correlación, fundamentadas en la integral de correlación, según se formuló en la subsección 4.2.2.

\subsubsection{Dimensión fractal}

La dimensión fractal es una medida descriptiva de la complejidad de un objeto autosemejante o autoafín, esto último en caso de que el escalamiento no sea uniforme en todas direcciones. ${ }^{2}$ En general la complejidad de una señal puede caracterizarse en los dominios temporal y frecuencial, así como en el espacio de estados del sistema del que se adquirió la señal. El análisis en el dominio frecuencial precisa de un aparato matemático derivado de Fourier (wavelets), mientras que el análisis en el espacio de estados requiere un encaje de los datos en un espacio de reconstrucción de dimensión normalmente alta, lo que dificulta la aplicación directa del método tradicional de conteo de cajas (box-counting method) no solo desde el punto de vista computacional, sino también de resultas de la incompatibilidad natural entre las unidades de las diferentes dimensiones, aparte de la presencia de ruido en los datos y la limitación de los mismos en cuanto a la cantidad capturada. En este sentido el dominio temporal brinda opciones del todo interesantes para cuantificar la complejidad de una señal.

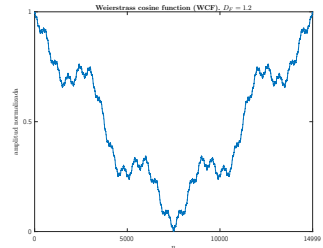

(a)

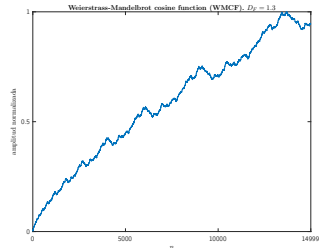

(b)

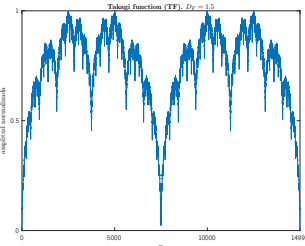

(c)

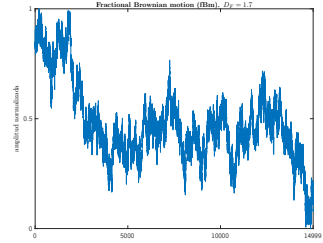

(d)

Figura 6.9: Evolución temporal de las señales de referencia para el cómputo de la dimensión fractal. En (a) se presenta una realización de la función coseno de Weierstrass (WCF) [Falconer, 1986], de $D_{F}=1,2$; en (b) una realización de la función coseno de Weierstrass-Mandelbrot (WMCF) [Feder, 2013], de $D_{F}=1,3$; en (c) una realización de la función de Takagi (TF) [Allaart and Kawamura, 2011], también conocida como función de Knopp, de $D_{F}=1,5$; en (d) una realización de un movimiento browniano fraccionario (fBm) [Flandrin, 1992], de $D_{F}=1,7$.

En el caso de una sucesión temporal, una curva planar (forma de onda), la dimensión fractal contiene información acerca de la complejidad estructural, en términos de ocurrencias de transiciones, de la evolución de la señal en el dominio del tiempo, esto es, la dimensión fractal, en este contexto, comporta una medida de la complejidad de la curva en el plano, o, dicho con otras palabras, la dimensión fractal determina el grado de llenado del espacio en un plano bidimiensional. Por consiguiente, la dimensión fractal $1<D_{F}<2$. La tabla 6.5 puntualiza los resultados recabados para las diferentes técnicas de cómputo de la dimensión fractal. Con arreglo a estos, y admitiendo que los métodos de Higuchi, de conteo de cajas de resolución múltiple, de conteo de cajas de resolución de longitud múltiple, y de retornos logarítmicos procuran mejores garantías, en correspondencia con los valores de estos métodos para

\footnotetext{
${ }^{2}$ Ningún objeto físico es realmente fractal porque no exhibe autosemejanza en todas las escalas.
} 
las señales de dimensión fractal conocida, se observa que las señales PPG presentan un carácter fractal no muy pronunciado, con un exponente de Hurst [Hurst, 1951] $H=2-D_{F}>0,5$ en todos los casos, lo que atribuye al proceso una memoria a largo plazo (persistencia) - de conformidad con el espectro de baja frecuencia de un típico ciclo PPG, como se precisa en [Ram et al., 2012]—, tanto más cuanto mayor sea $H$; desde una perspectiva geométrica la forma de onda de la señal, con un valor grande de $H$, identifica una evolución temporal muy suave, no tan irregular como cabría esperar en sistemas con $H=1 / 2$, exponente característico de un proceso browniano, totalmente aleatorio.

Tabla 6.5: Dimensión fractal $D_{F}$ de cinco señales PPG de cinco individuos diferentes. Con objeto de contrastar la validez de los métodos se han generado señales de dimensión fractal conocida (véase la figura 6.9), esto es, la función coseno de Weierstrass (WCF), de $D_{F}=1,2$, la función coseno de Weierstrass-Mandelbrot (WMCF), de $D_{F}=1,3$, la función de Takagi (TF), de $D_{F}=1,5$, y un movimiento browniano fraccionario $(\mathrm{fBm})$, de $D_{F}=1,7$. Las técnicas empleadas atienden al método de Higuchi [Higuchi, 1988], al método de Katz [Katz, 1988], al método de Sevcik [Sevcik, 2006], al método de conteo de cajas de resolución múltiple, o Multiresolution Boxcounting Method (MRBC) [Raghavendra and Dutt, 2010], al método de conteo de cajas de resolución de longitud múltiple, o Multiresolution Length Box-counting Method (MRLBC) [Raghavendra and Dutt, 2010], y al método de retornos logarítmicos, o Delayed log-returns [Stan et al., 2014].

\begin{tabular}{|lrrrccc|}
\hline \multicolumn{1}{c}{$\begin{array}{c}\text { señal } \\
\text { evaluada }\end{array}$} & \multicolumn{5}{c}{ dimensión fractal $D_{F}$} \\
\cline { 2 - 7 } & Higuchi & Katz & Sevcik & MRBC & MRLBC & log-returns \\
\hline Weierstrass cosine function (WCF) & 1,2004 & 1,2961 & 1,2459 & 1,1987 & 1,2031 & 1,1987 \\
\hline Weierstrass-Mandelbrot cosine function (WMCF) & 1,2775 & 1,2223 & 1,2569 & 1,2616 & 1,2760 & 1,3113 \\
\hline Takagi function (TF) & 1,5007 & 1,8217 & 1,3868 & 1,4934 & 1,5018 & 1,4877 \\
\hline Fractional Brownian motion (fBm) & 1,6866 & 2,4393 & 1,5651 & 1,6821 & 1,6839 & 1,6838 \\
\hline individuo núm. 1 (PPG1) & 1,0546 & 1,0724 & 1,3624 & 1,1372 & 1,0447 & 1,1168 \\
\hline individuo núm. 2 (PPG2) & 1,1658 & 1,0777 & 1,3699 & 1,1957 & 1,1033 & 1,1937 \\
\hline individuo núm. 3 (PPG3) & 1,1815 & 1,1181 & 1,4122 & 1,2333 & 1,1410 & 1,2112 \\
\hline individuo núm. 4 (PPG4) & 1,1126 & 1,0947 & 1,3878 & 1,1736 & 1,0901 & 1,1730 \\
\hline individuo núm. 5 (PPG5) & 1,1138 & 1,0715 & 1,3619 & 1,1719 & 1,0749 & 1,1655 \\
\hline \hline
\end{tabular}

\subsubsection{Dimensiones de información y de correlación}

A fin de relacionar la dimensión de información con la dimensión de correlación, se adopta, como una primera aproximación, el criterio por el que la dimensión de información, conforme a la conjetura de Kaplan-Yorke (remítase a 4.2.3.5), coincide con la dimensión de Lyapunov, referida como $D_{\mathrm{KY}}$ en la penúltima columna de la tabla 6.4.

Otra forma de calcular la dimensión de información se fundamenta en un algoritmo de masa fija, conforme a lo sugerido en 4.2.2.3, que divide el espacio en celdas de tamaño variable, de forma que cada celda contenga el mismo número de puntos, 
si bien los datos reportados, véase la figura 6.10, a modo de ejemplo, deben examinarse con ciertas reservas, dado que el estadístico en que se basa el algoritmo es muy sensible al ruido, muy común en datos experimentales, así como a la longitud de los datos recabados. De hecho, no en pocas ocasiones no es tan evidente adivinar una saturación de las curvas de dimensión en una región de escalas perfectamente delimitada.

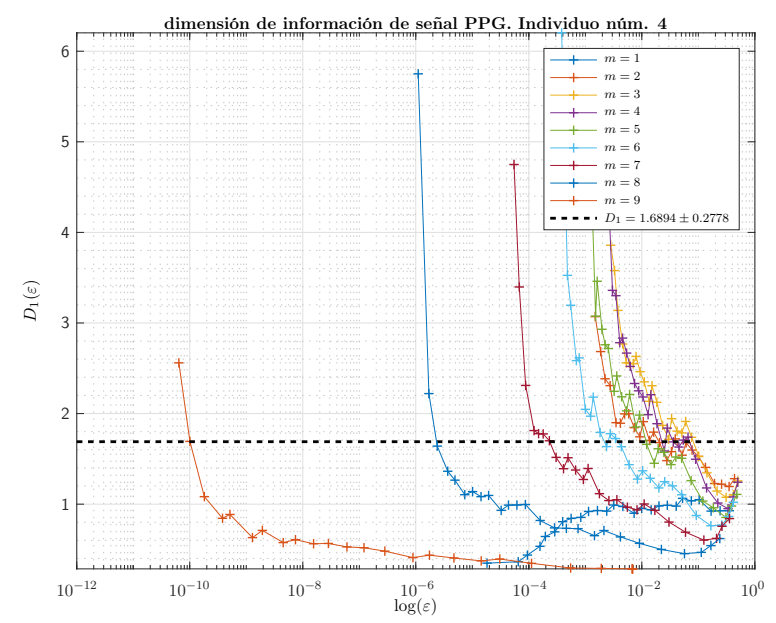

Figura 6.10: Dimensión de información de la señal PPG.

La figura 6.11 muestra las dimensiones de correlación de las señales de referencia, figuras 6.11a-6.11e, y de las cinco señales PPG, correspondientes a cinco individuos elegidos al azar, figuras 6.11f-6.11j.

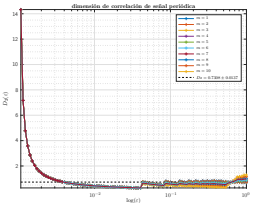

(a)

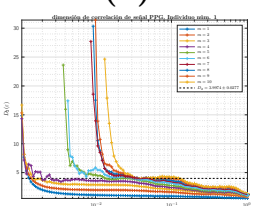

(f)

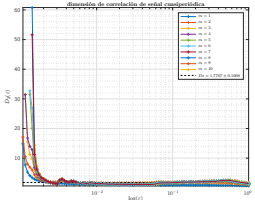

(b)

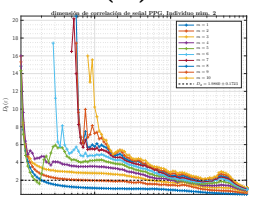

(g)

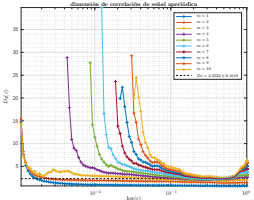

(c)

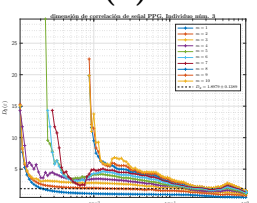

(h)

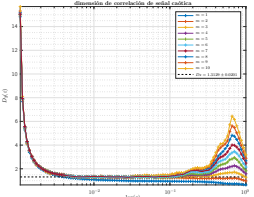

(d)

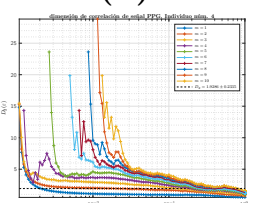

(i)

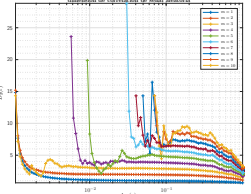

(e)

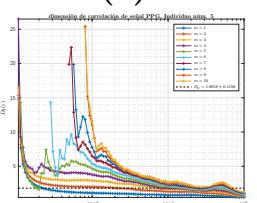

$(\mathbf{j})$

Figura 6.11: Dimensión de correlación $\left(D_{2}\right)$ de las señales de referencia (a)-(e) y de las señales PPG (f)-(j), adquiridas de cinco individuos elegidos aleatoriamente.

La tabla 6.6 recoge los resultados numéricos, que aluden, por una parte, a la dimensión de información, denotada como $D_{1}$, y, por otra parte, a la dimensión de correlación, identificada como $D_{2}$. Esta última ha sido extraída de las propias gráficas de la figura 6.11, mientras que la dimensión de información se atribuye a la conjetura de Kaplan-Yorke, según se expuso en 4.2.3.5, en virtud de la cual la dimen- 
sión de Lyapunov coincide con la dimensión de información, sirviendo de base para una primera aproximación cuando se pretende diferenciar ciertos comportamientos dinámicos.

Tabla 6.6: Dimensiones de información $\left(D_{1}\right)$ y de correlación $\left(D_{2}\right)$ de las señales de referencia y de las señales PPG, adquiridas de cinco individuos elegidos aleatoriamente.

\begin{tabular}{|lccc|}
\hline \hline \multicolumn{1}{|c}{ señal evaluada } & $m$ & $D_{1}$ & $D_{2}$ \\
\hline señal periódica & 2 & 1,0000 & $0,7308 \pm 0,0137$ \\
\hline señal cuasiperiódica & 5 & 3,4510 & $1,7767 \pm 0,1008$ \\
\hline señal aperiódica & 5 & 4,0751 & $2,3222 \pm 0,1618$ \\
\hline señal caótica & 2 & 1,2692 & $1,3129 \pm 0,0201$ \\
\hline señal aleatoria & 2 & 2,0000 & $2,0000 \pm 0,0000$ \\
\hline individuo núm. 1 (PPG1) & 5 & 2,9652 & $3,9974 \pm 0,0277$ \\
\hline individuo núm. 2 (PPG2) & 5 & 3,1015 & $1,9860 \pm 0,1723$ \\
\hline individuo núm. 3 (PPG3) & 5 & 3,1270 & $1,8979 \pm 0,1389$, \\
\hline individuo núm. 4 (PPG4) & 5 & 3,1469 & $1,9286 \pm 0,2335$ \\
\hline individuo núm. 5 (PPG5) & 5 & 3,0725 & $1,8058 \pm 0,1192$ \\
\hline \hline
\end{tabular}

Por un lado, la dimensión de correlación $D_{2}$, aparte de posibilitar la determinación del número de variables o ecuaciones que rigen la evolución dinámica del proceso físico considerado, como se ha concretado en la subsección 6.1.2, evalúa la dimensión fractal del espacio que ocupa un subconjunto de puntos escogidos al azar - la variación que experimenta la probabilidad promedio de que dos puntos cualesquiera, para una dimensión de reconstrucción del espacio de estados determinada, se mantengan próximos entre sí conforme aumenta la resolución de la partición del espacio-, lo que permite diferenciar un sistema aleatorio de un sistema determinista, si bien no siempre es capaz de resolver esta dicotomía, sobre todo cuando la dinámica determinista es demasiado compleja [Decoster and Mitchell, 1991]. Por otro lado, la dimensión de información $D_{1}$ igualmente constituye una medida de la dimensión fractal, pero no de un subconjunto de puntos escogidos aleatoriamente, sino de la distribución de probabilidad de la nube de puntos que conforman el espacio de estados del sistema dinámico. De este modo, la dimensión de información $D_{1}$ caracteriza la tasa de producción de información, a juzgar por la entropía de Shannon, a medida que se refina la partición del espacio de probabilidad. Con estos apuntes, y atendiendo a los datos recopilados en la tabla 6.6, se observa cómo las señales periódica y aleatoria carecen de una naturaleza fractal, a diferencia del resto de señales que, como ocurre con las señales PPG, se encasillarían en una configuración fractal. 


\subsubsection{Entropías}

Desde la perspectiva de una sucesión temporal, caracterizando la dinámica de un proceso físico, la entropía de Kolmogórov-Sinái, o $h_{\mathrm{KS}}$, como ya se introdujo en 4.2.3, comprende una medida de la incertidumbre o de la irregularidad en la transición entre sus diferentes estados, en conformidad con la definición introducida por Claude E. Shannon en 1948 [Shannon, 1948]. Una distribución de probabilidad de los estados similar imposibilita predecir el estado subsiguiente, increméntandose, por consiguiente, la entropía del sistema; si uno de sus estados presenta una probabilidad de uno, con el resto de estados manteniendo una probabilidad nula, el sistema evidencia una mínima entropía, y, por tanto, una mínima irregularidad. Si bien la entropía $h_{\mathrm{KS}}$ obedece a la definición formal de entropía métrica, para su cómputo se precisa de una partición generadora (generador), no siempre viable cuando únicamente se dispone de una sucesión temporal, con objeto de evaluar el supremo de la ecuación (4.58) sobre todas las posibles particiones del espacio de estados. Respecto a esta cuestión, y con la finalidad de conferir integridad al proceso de análisis dinámico de aquellos datos recabados a nivel experimental, en la última columna de la tabla 6.4 se proporciona una cota superior de la entropía $h_{\mathrm{KS}}$, de acuerdo con lo dictado en 4.2.3.5, esto es, si hubiere varios exponentes $\lambda_{i}$ positivos cada una de las direcciones inestables asociadas contribuye al efecto de expansión, y, de este modo, la identidad de Pesin asienta una primera relación entre la entropía $h_{\mathrm{KS}}$ y los exponentes de Lyapunov positivos [Pesin, 1977], esto es,

$$
h_{\mathrm{KS}} \leqslant \sum_{i: \lambda_{i}>0} \lambda_{i}
$$

Habitualmente, la identidad de Pesin, en sus diversas variantes - véanse las ecuaciones (4.70) y (4.71) —, se convierte en el procedimiento más eficaz para recabar una estimación veraz de la entropía $h_{\mathrm{KS}}$ de una sucesión temporal, dado que su cómputo directo se ve limitado por la insuficiencia de datos. Por todo lo antedicho se han venido proponiendo otras definiciones de entropía que - sin desatender el significado primario de entropía, a saber, cuantificar la regularidad presente en una sucesión temporal - recurren a la dinámica simbólica, de forma que, una vez transformada la sucesión temporal en símbolos, sea más factible obtener la partición generadora que maximiza el valor entrópico originario. En este trabajo se aplican las entropías de dispersión, aproximada, de muestra multiescala y de permutación, dada su profusión en el análisis de las características dinámicas de diversas señales biológicas, principalmente en configuraciones de tiempo real.

\subsubsection{Entropía de dispersión}

La entropía de dispersión, abreviadamente dispEn, y pormenorizada en [Rostaghi and Azami, 2016], cuantifica la regularidad de una sucesión temporal en términos de los cambios que experimentan la amplitud, la frecuencia, la potencia de ruido e incluso el ancho de banda de la señal considerada. Hoy día la entropía de dispersión está perfeccionándose con objeto de capturar más fehacientemente las sutilezas propias de las señales biológicas [Azami et al., 2017, Azami and Escudero, 2018]. La 
tabla 6.7 resume los resultados obtenidos tras computar la entropía de dispersión de las señales de referencia y de las cinco señales PPG, correspondientes a cinco individuos elegidos al azar, conforme a los parámetros $\tau$ y $m$ exhibidos en la tabla 6.2, de resultas de la metodología de reconstrucción del espacio de estados del proceso físico subyacente, recogida en el capítulo 3.

Tabla 6.7: Entropía de dispersión (dispEn). Sobre una sucesión temporal de 15.000 puntos se calcula la entropía de dispersión de bloques de datos de 1.500 puntos con un $80 \%$ de solapamiento entre los sucesivos bloques, procediéndose seguidamente a desvelar su media y desviación típica. El número de clases asciende a 8.

\begin{tabular}{|lccc|}
\hline \multicolumn{1}{|c}{ señal evaluada } & $\tau$ & $m$ & dispEn \\
\hline señal periódica & 1 & 2 & $0,6338 \pm 4,4900 \cdot 10^{-16}$ \\
\hline señal cuasiperiódica & 5 & 5 & $0,5895 \pm 0,0020$ \\
\hline señal aperiódica & 6 & 5 & $0,4620 \pm 0,0118$ \\
\hline señal caótica & 20 & 2 & $0,9668 \pm 0,0029$ \\
\hline señal aleatoria & 2 & 2 & $0,9821 \pm 0,0018$ \\
\hline individuo núm. 1 (PPG1) & 81 & 5 & $0,4380 \pm 0,0328$ \\
\hline individuo núm. 2 (PPG2) & 35 & 5 & $0,5025 \pm 0,0250$ \\
\hline individuo núm. 3 (PPG3) & 30 & 5 & $0,4818 \pm 0,0361$ \\
\hline individuo núm. 4 (PPG4) & 54 & 5 & $0,4425 \pm 0,0261$ \\
\hline individuo núm. 5 (PPG5) & 33 & 5 & $0,4431 \pm 0,0432$ \\
\hline \hline
\end{tabular}

Cabe subrayar que en el artículo originario [Rostaghi and Azami, 2016] todos los cálculos relativos a la entropía de dispersión se sirven de un retardo $\tau=1 \mathrm{y}$ de una dimensión $m$ variable, en función de las señales analizadas, pero en ningún momento se justifica el porqué de esos valores. En el presente trabajo se preserva la consistencia analítica de los datos, lo que reclama alimentar los algoritmos con los parámetros propios de la reconstrucción del espacio de estados. Con todo los valores numéricos de la tabla 6.7 apuntan a que las señales PPG se comportan como si de señales cuasiperiódicas y/o aperiódicas se trataran, mas en ningún caso como señales caóticas o aleatorias.

\subsubsection{Entropía aproximada}

La entropía, en lo tocante a los sistemas dinámicos, identifica la tasa de producción de información. No obstante, si el sistema bajo estudio viene caracterizado por una sucesión temporal, la escasez de datos y la presencia de ruido en los mismos dificultan una estimación correcta de la entropía. En este sentido la entropía aproximada, en siglas ApEn [Pincus, 1991, Pincus, 1995], comprende una medida de la 
regularidad de la sucesión temporal, estrechamente relacionada con la entropía $h_{\mathrm{KS}}$, en cuanto a la tasa de generación de nueva información a medida que evoluciona la dinámica del proceso físico subyacente. La entropía aproximada es susceptible de aplicación en el ámbito clínico, ${ }^{3}$ donde es frecuente disponer de señales biológicas de corta duración y muy contaminadas con ruido.

Tabla 6.8: Entropía aproximada (ApEn). Sobre una sucesión temporal de 15.000 puntos se calcula la entropía aproximada de bloques de datos de 1.500 puntos con un $80 \%$ de solapamiento entre los sucesivos bloques, procediéndose seguidamente a desvelar su media y desviación típica. El valor de $r$ asciende a un $20 \%$ de la desviación típica de la sucesión temporal estandarizada. En la tabla se muestra la desviación típica $\sigma$ de la sucesión temporal normalizada.

\begin{tabular}{|lccc|}
\hline \multicolumn{1}{|c}{ señal evaluada } & $\sigma$ & $m$ & ApEn \\
\hline señal periódica & 0,3005 & 2 & $0,0433 \pm 0,0000$ \\
\hline señal cuasiperiódica & 0,2502 & 5 & $0,2323 \pm 0,0020$ \\
\hline señal aperiódica & 0,3548 & 5 & $0,0665 \pm 0,0133$ \\
\hline señal caótica & 0,2813 & 2 & $0,4609 \pm 0,0049$ \\
\hline señal aleatoria & 0,2880 & 2 & $1,8520 \pm 0,0126$ \\
\hline individuo núm. 1 (PPG1) & 0,2149 & 5 & $0,0475 \pm 0,0047$ \\
\hline individuo núm. 2 (PPG2) & 0,1566 & 5 & $0,0884 \pm 0,0100$ \\
\hline individuo núm. 3 (PPG3) & 0,2103 & 5 & $0,0934 \pm 0,0130$ \\
\hline individuo núm. 4 (PPG4) & 0,2204 & 5 & $0,0502 \pm 0,0080$ \\
\hline individuo núm. 5 (PPG5) & 0,1660 & 5 & $0,0556 \pm 0,0137$ \\
\hline \hline
\end{tabular}

El marco teórico en el que se basó Steven M. Pincus para la confección de la entropía aproximada se remonta a los trabajos efectuados por Peter Grassberger e Itamar Procaccia [Grassberger and Procaccia, 1983], y por Jean-Pierre Eckmann y David Ruelle [Eckmann and Ruelle, 1985]. Un valor pequeño de ApEn refleja un alto índice de regularidad. A pesar de su indiscutible utilidad en la detección de diferentes comportamientos dinámicos, presenta cierto sesgo o inconsistencia cuando se dispone de sucesiones temporales con pocos datos [Richman and Moorman, 2000]. Es por esto que surgió la entropía de muestra, derivada de los estudios emprendidos por Peter Grassberger y colaboradores [Grassberger and Procaccia, 1983, Ben-Mizrachi et al., 1984, Grassberger, 1988, Grassberger et al., 1991], muy común en el procesamiento de imágenes, y sus variantes, como la entropía de muestra multiescala, abordada más adelante, a fin de reparar el inconveniente del sesgo, aunque adolece, al igual que la

\footnotetext{
${ }^{3}$ Estudios cardiovasculares al respecto son prolíficos en la literatura médica, al igual que actualmente, y de forma más metódica, en lo referente al análisis de la información contenida en un electroencefalograma (EEG) [Mesin, 2018].
} 
propia entropía aproximada, de una carga computacional intensa que la descarta irremediablemente, máxime con sucesiones temporales de cierta longitud, para su participación en aplicaciones de tiempo real.

La tabla 6.8 resume los resultados obtenidos tras computar la entropía aproximada de las señales de referencia y de las cinco señales PPG, correspondientes a cinco individuos elegidos al azar, conforme al parámetro $m$ exhibido en la tabla 6.2, de resultas de la metodología de reconstrucción del espacio de estados del proceso físico subyacente, recogida en el capítulo 3. En todo caso los valores númericos de la tabla 6.8 sugieren una ligera tendencia, moderada por la cercanía del comportamiento periódico, de las señales PPG hacia la aperiodicidad, y en menor medida hacia la cuasiperiodicidad, pero de ninguna manera hacia la caoticidad o aleatoriedad, diferencia acentuada gráficamente en las figuras 6.12a-6.12b.

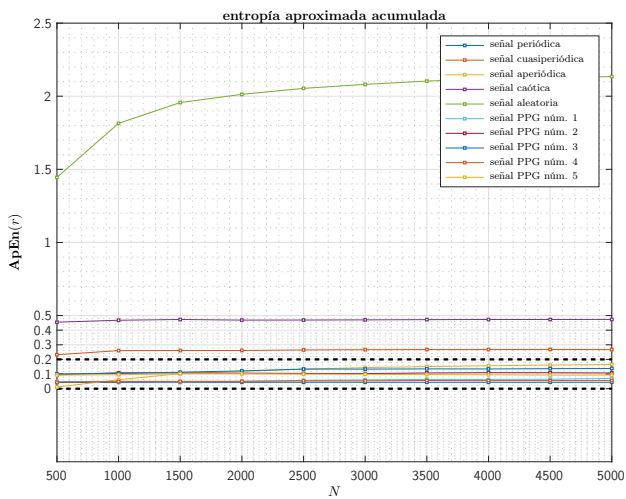

(a)

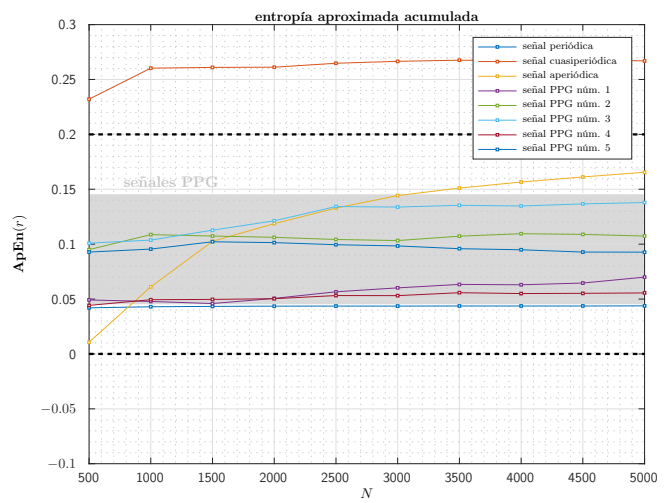

(b)

Figura 6.12: Tendencia de la entropía aproximada (ApEn), cuando $r=0,2$, conforme se van acumulando bloques de datos de longitud 500, de las señales de referencia y de las señales PPG, adquiridas de cinco individuos elegidos aleatoriamente. En (a) se muestran todas las señales; en (b) únicamente se reflejan las señales PPG en comparación con aquellas señales que presentan similares tendencias entrópicas en orden de magnitud.

La figura 6.12 compendia la tendencia que experimenta la entropía aproximada, tanto para las señales de referencia como para las señales PPG, conforme se anexionan más datos a las sucesiones temporales, como si de fuentes de información se tratara. De esta forma resulta más sencillo circunscribir el comportamiento dinámico de las señales PPG en contraste con las señales de referencia, cuyo comportamiento es conocido a priori. En la figura 6.12a se muestran, en un primer examen, todas las señales, tanto las de referencia como las señales PPG; en la figura 6.12b, a modo de ampliación, se ilustran únicamente las señales PPG y las señales de referencia con entropías más afines a aquellas.

\subsubsection{Entropía de muestra multiescala}

La entropía de muestra mutiescala [Costa et al., 2005], multescEn de forma abreviada, se ideó, sobre la base de la entropía de muestra, principalmente para dis- 
criminar las componentes determinista y estocástica de muchos sistemas fisiológicos, dado que estos están regulados por mecanismos subyacentes que interactúan a diferentes escalas espacio-temporales. Por tanto, la entropía de muestra multiescala se asienta sobre tres hipótesis de partida:

1. La complejidad de los sistemas fisiológicos refleja su habilidad para adaptarse a un entorno circundante en perpetuo cambio.

2. Los sistemas fisiológicos, para su correcto funcionamiento, necesitan operar a diferentes escalas espacio-temporales.

3. Diversas patologías, así como el proceso de envejecimiento, reducen la capacidad adaptativa del sistema afectado, lo que redunda en una pérdida de complejidad.

La figura 6.13 resume la tendencia que experimenta la entropía de muestra multiescala, tanto para las señales de referencia como para las señales PPG, conforme incrementa el factor de escala. Sobre una misma figura resulta más aparente demarcar el comportamiento dinámico de las señales PPG en contraste con las señales de referencia, cuyo comportamiento es conocido a priori. En la figura 6.13a se muestran, en un primer examen, todas las señales, tanto las de referencia como las señales PPG; en la figura 6.13b, a modo de ampliación, se ilustran únicamente las señales PPG y las señales de referencia con entropías análogas en orden de magnitud, dentro de un rango determinado.

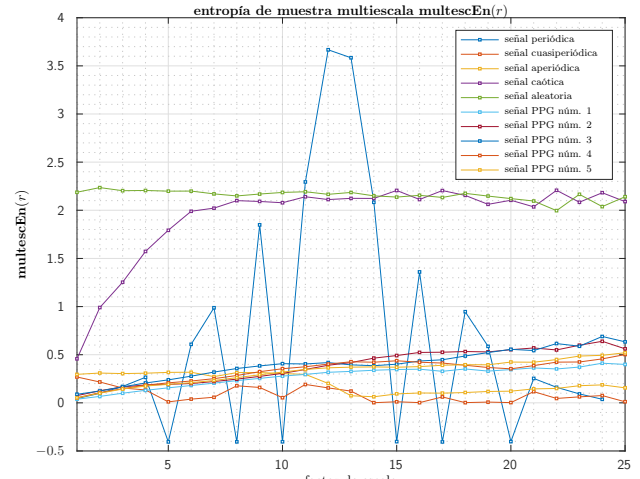

(a)

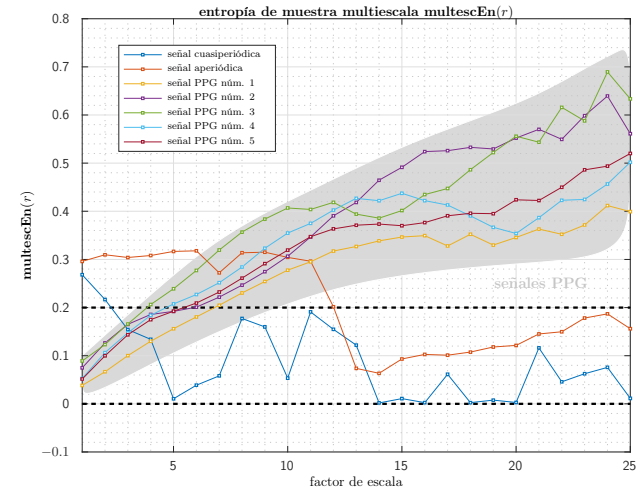

(b)

Figura 6.13: Evolución de la entropía de muestra multiescala (multescEn), cuando $r=0,2$, para diferentes factores de escala, de las señales de referencia y de las señales PPG, adquiridas de cinco individuos elegidos aleatoriamente. En (a) se muestran todas las señales; en (b) únicamente se reflejan las señales PPG (su tendencia aparece resaltada) en comparación con aquellas señales que presentan similares tendencias entrópicas en orden de magnitud.

La tabla 6.9 resume los resultados obtenidos tras computar la entropía de muestra multiescala de las señales de referencia y de las cinco señales PPG, correspondientes a cinco individuos elegidos al azar, conforme al parámetro $m$ exhibido en la tabla 6.2. 
Además, en la tabla 6.9 se incluyen los tiempos de cómputo de la entropía de muestra multiescala, evidenciándose la carga computacional de la entropía de muestra, como se adelantó anteriormente. No obstante, dado que los valores numéricos de la entropía multescEn de la tabla 6.9 han sido promediados para todos los factores de escala, únicamente resultan evidentes las diferencias cuantitativas entre las señales PPG y las señales caótica y aleatoria, como también refleja la figura 6.13a.

Tabla 6.9: Entropía de muestra multiescala (multescEn). Sobre una sucesión temporal de 15.000 puntos se calcula la entropía de muestra para 25 factores de escala, procediéndose seguidamente a desvelar su media y desviación típica. El valor de $r$ asciende a un $20 \%$ de la desviación típica de la sucesión temporal estandarizada.

\begin{tabular}{|c|c|c|c|}
\hline señal evaluada & $\mathbf{t}_{\text {cómputo }}(\mathrm{en} \mathrm{s})$ & $m$ & multescEn \\
\hline señal periódica & $1 \mathrm{~h} 19 \min 10 \mathrm{~s}(4.750)$ & 2 & $0,3570 \pm 0,7118$ \\
\hline señal cuasiperiódica & $1 \mathrm{~h} 13 \min 52 \mathrm{~s}(4.432)$ & 5 & $0,0856 \pm 0,0782$ \\
\hline señal aperiódica & $1 \mathrm{~h} 5 \min 11 \mathrm{~s}(3.911)$ & 5 & $0,2061 \pm 0,0950$ \\
\hline señal caótica & $1 \mathrm{~h} 4 \min 29 \mathrm{~s}(3.869)$ & 2 & $1,9315 \pm 0,4274$ \\
\hline señal aleatoria & $1 \mathrm{~h} 13 \min 26 \mathrm{~s}(4.406)$ & 2 & $2,1561 \pm 0,0521$ \\
\hline individuo núm. 1 (PPG1) & $1 \mathrm{~h} 13 \min 7 \mathrm{~s}(4.387)$ & 5 & $0,2746 \pm 0,1070$ \\
\hline individuo núm. 2 (PPG2) & $1 \mathrm{~h} 11 \min 54 \mathrm{~s}(4.314)$ & 5 & $0,3875 \pm 0,1731$ \\
\hline individuo núm. 3 (PPG3) & $1 \mathrm{~h} 12 \min 21 \mathrm{~s}(4.341)$ & 5 & $0,4035 \pm 0,1590$ \\
\hline individuo núm. 4 (PPG4) & $1 \mathrm{~h} 12 \min 37 \mathrm{~s}(4.357)$ & 5 & $0,3337 \pm 0,1189$ \\
\hline individuo núm. 5 (PPG5) & $1 \mathrm{~h} 6 \min 21 \mathrm{~s}(3.981)$ & 5 & $0,3262 \pm 0,1256$ \\
\hline
\end{tabular}

Para dilucidar el comportamiento de las señales PPG, en contraposición con las señales periódica, cuasiperiódica y aperiódica, se recurre a la figura 6.13b, donde se aprecia claramente cómo las señales PPG propenden hacia comportamientos caóticos, o aleatorios, a medida que aumenta el factor de escala, esto es, conforme se examina el comportamiento de las señales PPG a baja resolución (bajas frecuencias) se revela una clara tendencia hacia el caos, o hacia la aleatoriedad, si es el efecto del ruido el factor predominante, lo que, en el primer caso, respaldaría la complejidad inherente al acoplamiento entre los subsistemas respiratorio y cardiovascular. De hecho, en la actualidad la entropía de muestra multiescala está ofreciendo prometedores resultados en la detección de patologías cardiovasculares e incluso de alteraciones genéticas en la secuencia del ADN [Mesin, 2018]. Tanto es así que la noción de multiescala se está extendiendo a otro tipo de entropías en un intento por retener las diferentes dinámicas superpuestas en los procesos fisiológicos, que, como es bien sabido, no constituyen sistemas cerrados, sino más bien abiertos e interactuando recíprocamente entre sí. 


\subsubsection{Entropía de permutación}

La complejidad de un sistema dinámico puede medirse mediante la entropía métrica, también denominada entropía de Kolmogórov-Sinái, $h_{\mathrm{KS}}$, el mayor exponente de Lyapunov, o la dimensión de correlación, si bien no resulta fácil estimar su valor, o magnitudes similares, a partir de sucesiones temporales del mundo real y de longitud finita. Las diferentes soluciones algorítmicas fracasan tan pronto el ruido hace acto de presencia, y, normalmente, los resultados que proporcionan no son fiel reflejo de lo que sucede realmente, llevando a conclusiones erróneas.

Con esta panorámica, en 2002 Christoph Bandt y Bernd Pompe introdujeron el concepto de entropía de permutación [Bandt and Pompe, 2002], o permutEn para acortar, con objeto de estimar la complejidad de una sucesión temporal del mundo real. La entropía de permutación de una sucesión temporal comprende la entropía de Shannon de la distribución de patrones ordinales presentes en la sucesión temporal. Los patrones ordinales, que describen relaciones de orden en las componentes de un vector, se codifican por medio de permutaciones. Sea $\Pi_{d}$ el conjunto de permutaciones de $\{0,1, \ldots, d\}$, para $d \in \mathbb{N}$. Se dice que un vector $\left(v_{0}, v_{1}, \ldots, v_{d}\right) \in \mathbb{R}^{d+1}$ tiene un patrón ordinal $\pi=\left(r_{0}, r_{1}, \ldots, r_{d}\right) \in \Pi_{d}$, si $v_{r_{0}} \geqslant v_{r_{1}} \geqslant \cdots \geqslant v_{r_{d-1}} \geqslant v_{r_{d}}$, y $r_{l-1}>r_{l}$ sii $v_{r_{l-1}}>v_{r_{l}}$ [Keller et al., 2017].

Sobre la base de las relaciones de orden que se establecen entre los elementos que componen una sucesión temporal, la entropía de permutación se relaciona con $h_{\mathrm{KS}}$; de hecho, en muchos sistemas dinámicos la entropía de permutación coincide con $h_{\mathrm{KS}}$ [Keller et al., 2012].

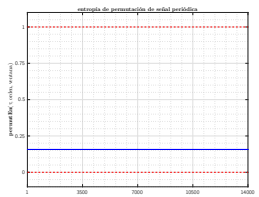

(a)

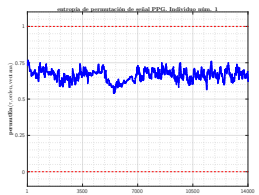

(f)

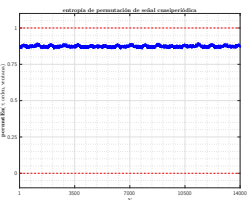

(b)

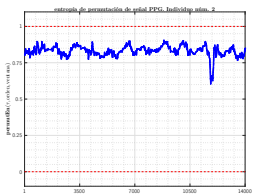

$(\mathrm{g})$

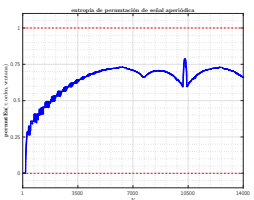

(c)

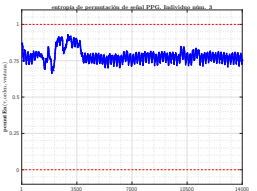

(h)

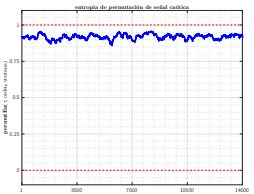

(d)

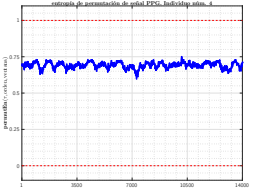

(i)

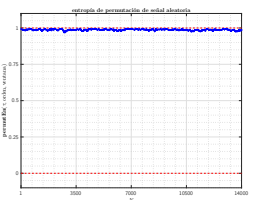

(e)

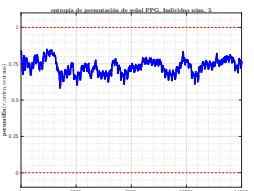

$(\mathbf{j})$

Figura 6.14: Entropía de permutación (permutEn) de las señales de referencia (a)-(e) y de las señales PPG (f)-(j), adquiridas de cinco individuos elegidos aleatoriamente.

Por otro lado, la entropía de permutación se evalúa vía la entropía de permutación empírica [Bandt and Pompe, 2002, Keller et al., 2007], conceptualmente muy simple y algorítmicamente muy rápida [Unakafova and Keller, 2013], si bien las relaciones de orden ignoran las variaciones de amplitud presentes en los datos [Zanin et al., 2012]. En este sentido la entropía de permutación empírica se muestra muy robusta frente al ruido dinámico y observacional [Bandt and Pompe, 2002, Amigó, 2010]. Finalmente, la entropía de permutación ofrece la posibilidad de comparar diferentes sucesiones 
temporales, de idéntica longitud, de una manera muy sencilla y estándar. Dada su versatilidad y robustez no resulta extraño advertir su presencia en campos científicos y experimentales tan diversos como el ámbito clínico de las señales biológicas o el mundo de las finanzas [Amigó, 2010, Graff et al., 2013, Keller et al., 2017]. Además, se ha posicionado recientemente como un factor discriminante apto en tareas de segmentación y clasificación de estados patológicos a partir de los típicos indicadores clínicos [Cuesta-Frau et al., 2018].

Tabla 6.10: Entropía de permutación (permutEn). Sobre una sucesión temporal de 15.000 puntos se calcula la entropía de permutación de ventanas temporales de 250 puntos, procediéndose seguidamente a desvelar su media y desviación típica. El orden de los patrones ordinales asciende a 3.

\begin{tabular}{|lcc|}
\hline \multicolumn{1}{|c}{ señal evaluada } & $\tau$ & permutEn \\
\hline señal periódica & 1 & $0,1569 \pm 3,4862 \cdot 10^{-14}$ \\
\hline señal cuasiperiódica & 5 & $0,8703 \pm 0,0069$ \\
\hline señal aperiódica & 6 & $0,6350 \pm 0,1252$ \\
\hline señal caótica & 20 & $0,9192 \pm 0,0165$ \\
\hline señal aleatoria & 2 & $0,9871 \pm 0,0048$ \\
\hline individuo núm. 1 (PPG1) & 81 & $0,6625 \pm 0,0379$ \\
\hline individuo núm. 2 (PPG2) & 35 & $0,8323 \pm 0,0348$ \\
\hline individuo núm. 3 (PPG3) & 30 & $0,7833 \pm 0,0427$ \\
\hline individuo núm. 4 (PPG4) & 54 & $0,6921 \pm 0,0210$ \\
\hline individuo núm. 5 (PPG5) & 33 & $0,7299 \pm 0,0482$ \\
\hline \hline
\end{tabular}

La figura 6.14 muestra la entropía de permutación de las señales de referencia, figuras 6.14a-6.14e, y de las cinco señales PPG, correspondientes a cinco individuos elegidos al azar, figuras 6.14f-6.14j. Los valores numéricos son recogidos en la tabla 6.10, respetándose en todo momento, en aras de la consistencia, el parámetro $\tau$ exhibido en la tabla 6.2, de resultas de la metodología de reconstrucción del espacio de estados del proceso físico subyacente, recogida en el capítulo 3. Como puede advertirse no es fácil intuir si las señales PPG presentan un comportamiento cuasiperiódico o aperiódico, salvo si se considera el crecimiento exponencial que experimenta inicialmente la señal aperiódica, ostensible en la figura $6.15 \mathrm{c}$, pero en ningún caso su comportamiento podría asociarse al característico de las señales periódica, caótica o aleatoria.

La figura 6.15 condensa la tendencia que experimenta la entropía de permutación, tanto para las señales de referencia, figuras 6.15a-6.15e, como para las señales PPG, figuras $6.15 \mathrm{f}-6.15 \mathrm{j}$, conforme se anexionan más datos a las sucesiones temporales, como si de fuentes de información se tratara. Con esta composición es posible detectar 
cambios sutiles en el comportamiento dinámico de las señales PPG, que podrían revelar transformaciones funcionales en los subsistemas que conforman la dinámica de un ciclo PPG.

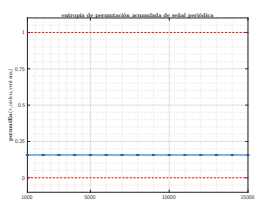

(a)

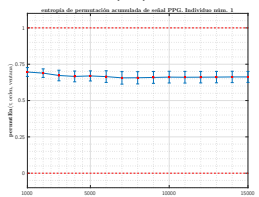

(f)

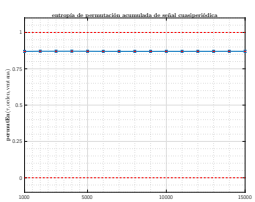

(b)

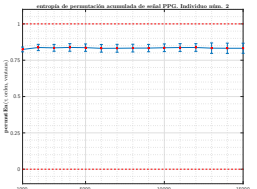

$(\mathrm{g})$

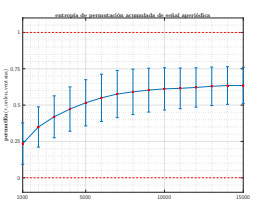

(c)

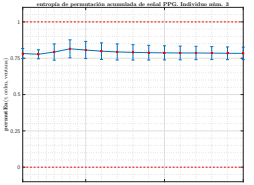

(h)

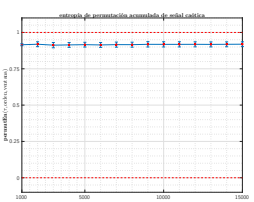

(d)

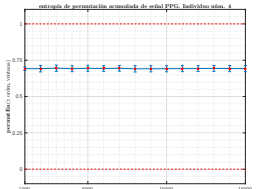

(i)

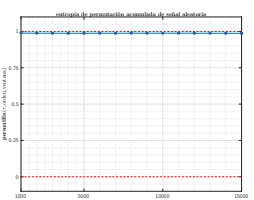

(e)

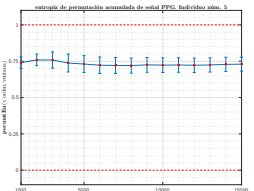

$(\mathbf{j})$

Figura 6.15: Tendencia de la entropía de permutación (permutEn), conforme se van acumulando bloques de datos de longitud 1.000, de las señales de referencia (a)-(e) y de las señales PPG (f)-(j), adquiridas de cinco individuos elegidos aleatoriamente.

La figura 6.16 ilustra, en una sola representación, la entropía de permutación acumulada de todas las señales, tanto las de referencia como las señales PPG. A la luz de esta gráfica las señales PPG adoptan un comportamiento entrópico comprendido entre los exhibidos por una señal aperiódica y una señal cuasiperiódica, y siempre distanciado del comportamiento caótico y aleatorio.

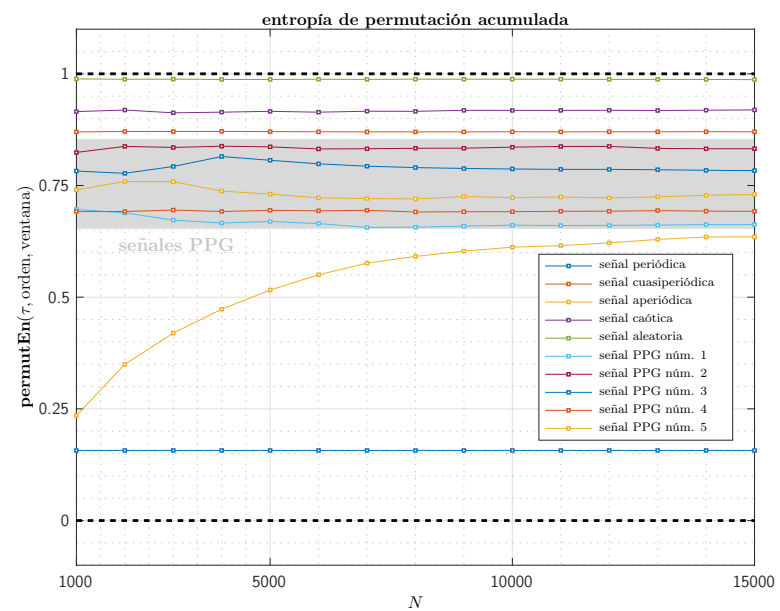

Figura 6.16: Tendencia de la entropía de permutación (permutEn), conforme se van acumulando bloques de datos de longitud 1.000, de las señales de referencia y de las señales PPG, adquiridas de cinco individuos elegidos aleatoriamente; aparecen resaltadas las señales PPG, de forma que se reconozca notoriamente su naturaleza, contenida en el rango comprendido entre la aperiodicidad y el carácter cuasiperiódico. 


\subsection{Técnicas de medida de la complejidad}

Como se adelantó en la sección 4.3, las dos técnicas que se introducen en este trabajo, con el propósito de interpretar la complejidad de un sistema dinámico y así recurrir a las herramientas de análisis apropiadas, se corresponden con el test 0-1, en esencia por su fácil implementación, independientemente de la información disponible del sistema físico subyacente, y con el algoritmo de análisis de complejidad de Lempel-Ziv, especialmente útil en aplicaciones biomédicas como una medida para estimar la complejidad de señales en tiempo discreto.

\subsubsection{Test $0-1$}

El test 0-1, propuesto y examinado en [Gottwald and Melbourne, 2004, Gottwald and Melbourne, 2005, Gottwald and Melbourne, 2009a], permite distinguir, con poca carga computacional, una dinámica regular, periódica o cuasiperiódica, de una dinámica caótica, operando directamente sobre la sucesión temporal, incluso corrompida por efecto del ruido, sin recurrir a un preprocesamiento previo de los datos, como sucede con la reconstrucción del espacio de estados.

La figura 6.17 ilustra el modo difusivo que presentan las señales de referencia, figuras 6.17a-6.17e, y las cinco señales PPG, correspondientes a cinco individuos elegidos al azar, figuras 6.17f-6.17j.

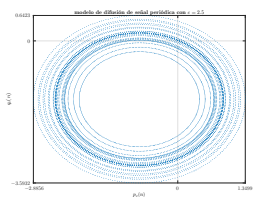

(a)

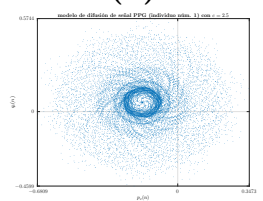

(f)

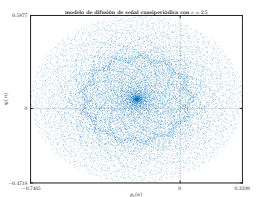

(b)

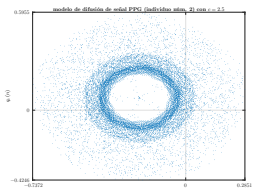

$(\mathrm{g})$

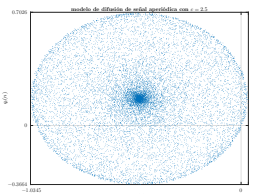

(c)

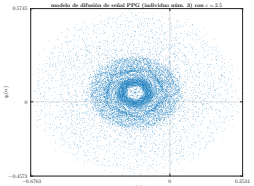

(h)

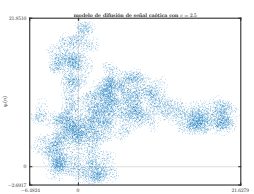

(d)

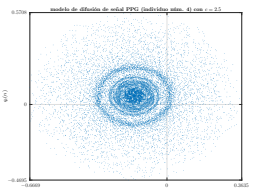

(i)

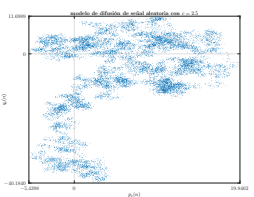

(e)

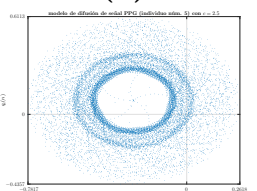

(j)

Figura 6.17: Modelo de difusión, de acuerdo con sucesiones temporales de 15.000 puntos, para un valor de $c=2,5$, de las señales de referencia (a)-(e) y de las señales PPG (f)-(j), adquiridas de cinco individuos elegidos aleatoriamente.

A primera vista es manifiesta la diferencia que se transluce entre las señales PPG y las señales caótica y aleatoria, figuras $6.17 \mathrm{~d}-6.17 \mathrm{e}$, no solo en cuanto al rango dinámico que conlleva el proceso de difusión, muy explosivo en las señales caótica y aleatoria, sino en cuanto a su estructura geométrica. Las señales PPG, muy confinadas en el espacio, presentan una región central que conjuga una composición transitiva, desde una ordenación periódica, dado el carácter recurrente de los ciclos PPG, pasando por estadíos aperiódicos, conforme se aleja la configuración del centroide estructural, que en su posible transición hacia el caos se ve detenida por la acción cuasiperiódica, característica de un atractor SNA, en el cinturón perimetral. 
Para analizar el comportamiento difusivo, o no difusivo, de un sistema dinámico, representado por una sucesión temporal, se computa $M_{c}(n)$, o, en su defecto, $D_{c}(n)$, pues exhibe menos varianza que $M_{c}(n)$. Así, en la figura 6.18 se muestra el comportamiento difusivo que manifiestan las señales de referencia, figuras 6.18a6.18e, y las cinco señales PPG, correspondientes a cinco individuos elegidos al azar, figuras $6.18 \mathrm{f}-6.18 \mathrm{j}$.

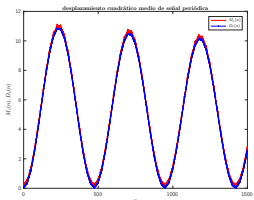

(a)

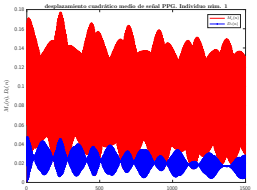

(f)

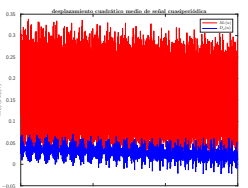

(b)

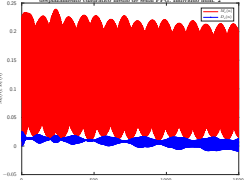

$(\mathrm{g})$

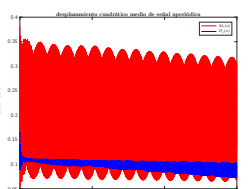

(c)

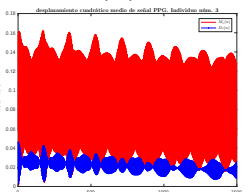

(h)

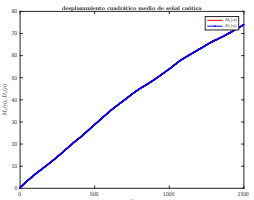

(d)

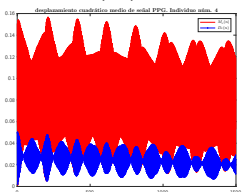

(i)

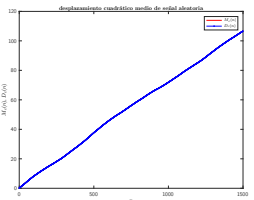

(e)

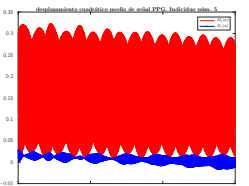

(j)

Figura 6.18: Capacidad difusiva, de acuerdo con sucesiones temporales de 15.000 puntos, de las señales de referencia (a)-(e) y de las señales PPG (f)-(j), adquiridas de cinco individuos elegidos aleatoriamente. En (d)-(e) las curvas de $M_{c}(n)$ y $D_{c}(n)$ no se aprecian pues al coincidir sus valores figuran superpuestas.

En las figuras 6.18d-6.18e se distingue claramente la tasa de crecimiento lineal característica de aquellas dinámicas con aspecto más «errático», esto es, reflejan de algún modo la facilidad con la que la dinámica se mueve en el espacio, en este caso bidimensional por definición del método, en el que se extienden los estados que conforman el potencial atractor. ${ }^{4}$ Con todo, el comportamiento difusivo de las señales PPG guarda un vínculo más estrecho con el exhibido por las señales cuasiperiódica y aperiódica, figuras 6.18b-6.18c, si bien la tendencia negativa de las trazas de $D_{c}(n)$, contemporizando oscilaciones más positivas o expansivas, propias de la acción aperiódica, y oscilaciones menos positivas o restrictivas, a causa de la actuación cuasiperiódica, revela una mecánica difusiva que combina ambas dinámicas funcionales, en las que a veces el engranaje operativo, siempre confinado en el espacio, concede más margen al extremo aperiódico.

La tasa de crecimiento asintótico $K_{c}$ de $M_{c}(n)$, o de su versión modificada $D_{c}(n)$, permite diferenciar un comportamiento regular de un comportamiento caótico. Dado que el método de correlación ofrece mejores resultados que el método de regresión [Gottwald and Melbourne, 2009b], se opta por el primero; por consiguiente, el coeficiente $K_{c}$ evalúa el grado de correlación lineal entre $M_{c}(n)$, o $D_{c}(n)$, y un crecimiento lineal, propio de dinámicas «erráticas». La figura 6.19 identifica el coeficiente difusivo que evidencian las señales de referencia, figuras 6.19a-6.19e, y las cinco señales PPG, correspondientes a cinco individuos elegidos al azar, figuras 6.19f-6.19j.

\footnotetext{
${ }^{4}$ En dinámica de fluidos representa la facilidad con que cada soluto se mueve en un disolvente determinado.
} 
A fin de establecer una comparativa entre las señales de referencia y las señales PPG, se estima $K_{m}=$ media $\left(K_{c}\right)$ alrededor de la región central de los valores de $K_{c}$ calculados, como así se indica en las figuras 6.19b-6.19c y en las figuras 6.19f-6.19j.

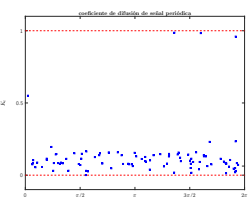

(a)

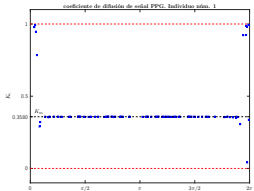

(f)

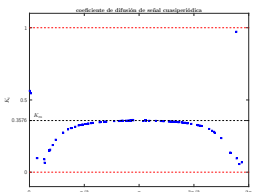

(b)

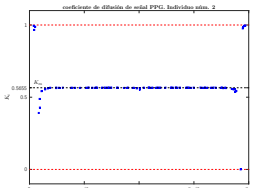

(g)

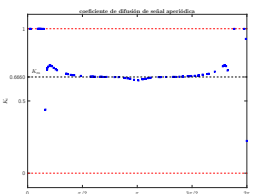

(c)

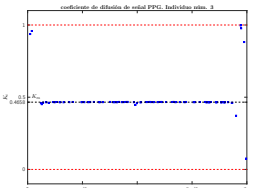

(h)

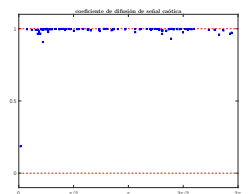

(d)

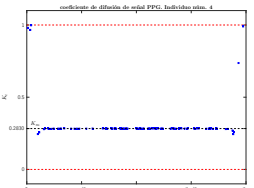

(i)

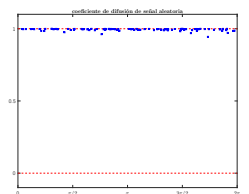

(e)

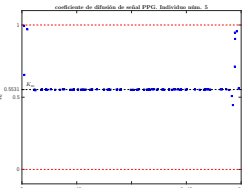

$(\mathbf{j})$

Figura 6.19: Coeficiente de difusión, de acuerdo con sucesiones temporales de 15.000 puntos, para 100 valores de $c$ elegidos al azar, de las señales de referencia (a)-(e) y de las señales PPG (f)-(j), adquiridas de cinco individuos elegidos aleatoriamente.

Las señales PPG presentan un coeficiente difusivo, siguiendo lo apuntado anteriormente, que fluctúa entre el puramente cuasiperiódico y el estrictamente aperiódico, respondiendo a un acoplamiento, presumiblemente no lineal, derivado de la interacción mutua entre los diferentes subsistemas, en suma cardiovascular y respiratorio, reflejados en un ciclo PPG. La posibilidad de evaluar mediante el test $0-1$ ventanas de intermitencia [Gottwald and Melbourne, 2016, Sviridova et al., 2018] o rutas de «fractalización» hacia SNA [Gopal et al., 2013] abre todo un abanico de alternativas a la hora de profundizar en la comprensión e interpretación de los mecanismos funcionales que potencian o inhiben el acoplamiento entre estos subsistemas y cómo pueden asociarse con estados patológicos del individuo.

\subsubsection{Complejidad de Lempel-Ziv}

En calidad de medida derivada de la noción de complejidad de Kolmogórov [Kolmogorov, 1968], ${ }^{5}$ la complejidad de Lempel-Ziv posibilita el cómputo de la cantidad de información presente en una sucesión de símbolos [Lempel and Ziv, 1976]. Los diferentes algoritmos pretenden descubrir, sobre la base de una codificación binaria, la complejidad de la estructura dinámica de las diversas sucesiones temporales. En los últimos años la complejidad de Lempel-Ziv ha recibido especial atención en el entorno biomédico [Alcaraz, 2018], como, por ejemplo, en la detección de enfermedades neurodegenerativas [Ruffini et al., 2018], en el análisis de la actividad cerebral con el envejecimiento experimentado [Shumbayawonda et al., 2018], etc.

Dado que en el ámbito de las señales biológicas la sucesión temporal es convertida a una sucesión binaria (alfabeto finito), conforme a unas reglas de transformación, se proponen tres pautas de conversión a formato binario, procedimientos 6.1, 6.2 y

\footnotetext{
${ }^{5}$ También denominada complejidad estocástica o entropía algorítmica, pues comporta una medida de los recursos necesarios para describir una cierta cantidad de información.
} 
6.3, puesto que dependiendo del patrón aplicado puede dirigirse la búsqueda de la complejidad en el dominio más conveniente.
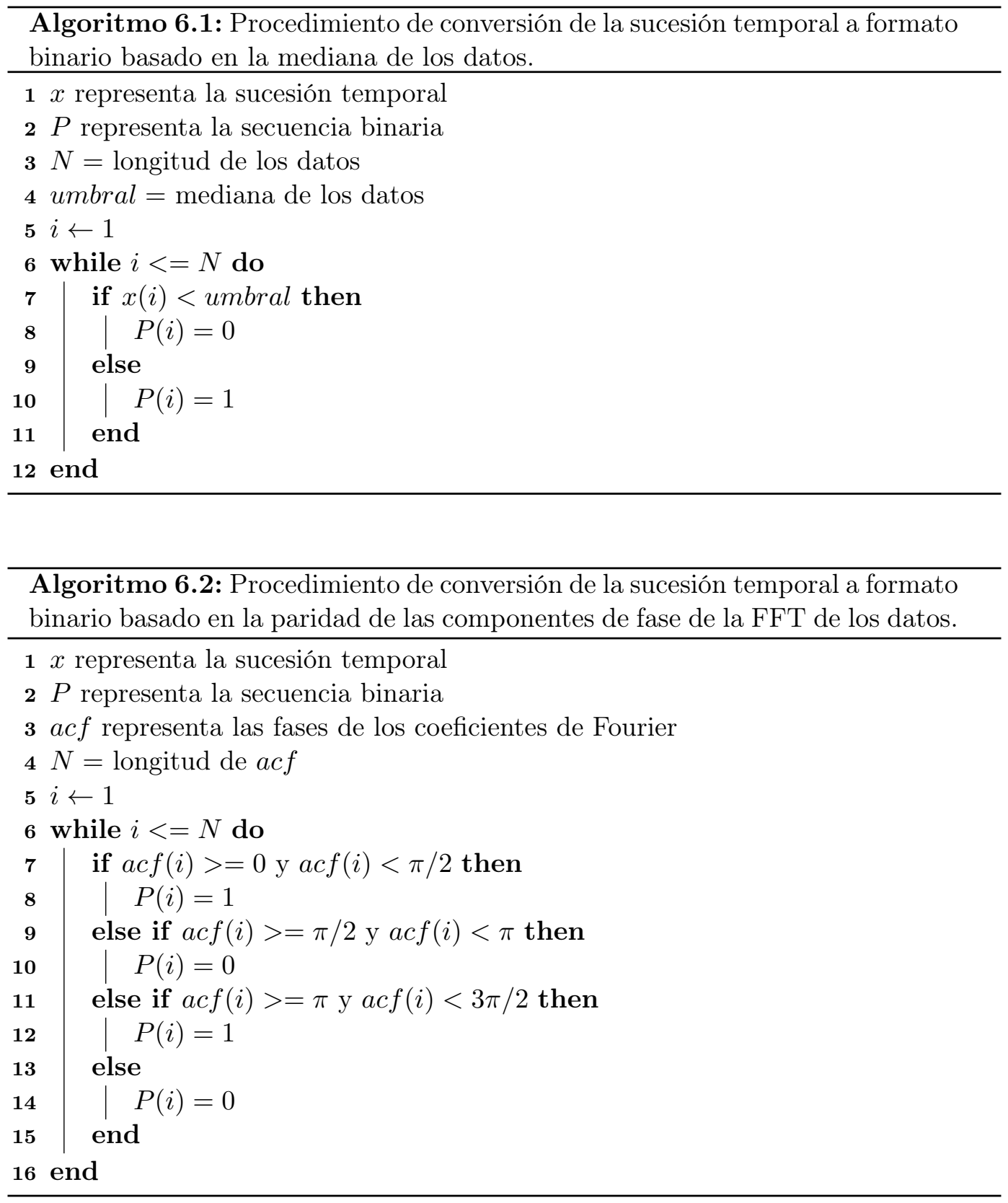

Así, a modo de primera tentativa, dada su habitualidad en el entorno biomédico, se opta por un dominio más estadístico, como refleja el algoritmo 6.1, en el que la mediana de los datos constituye la línea divisoria entre un ' 1 ' y un ' 0 '. Otras alternativas se centran en el dominio fásico, como el algoritmo 6.2, con arreglo a la transformada de Fourier, o, incluso, en el contexto de las variaciones que experimentan las amplitudes dentro de cada vector de estado reconstruido, como refiere el algoritmo 6.3, atendiendo a la metodología de reconstrucción del espacio de estados 
del proceso físico subyacente, recogida extensamente en el capítulo 3.

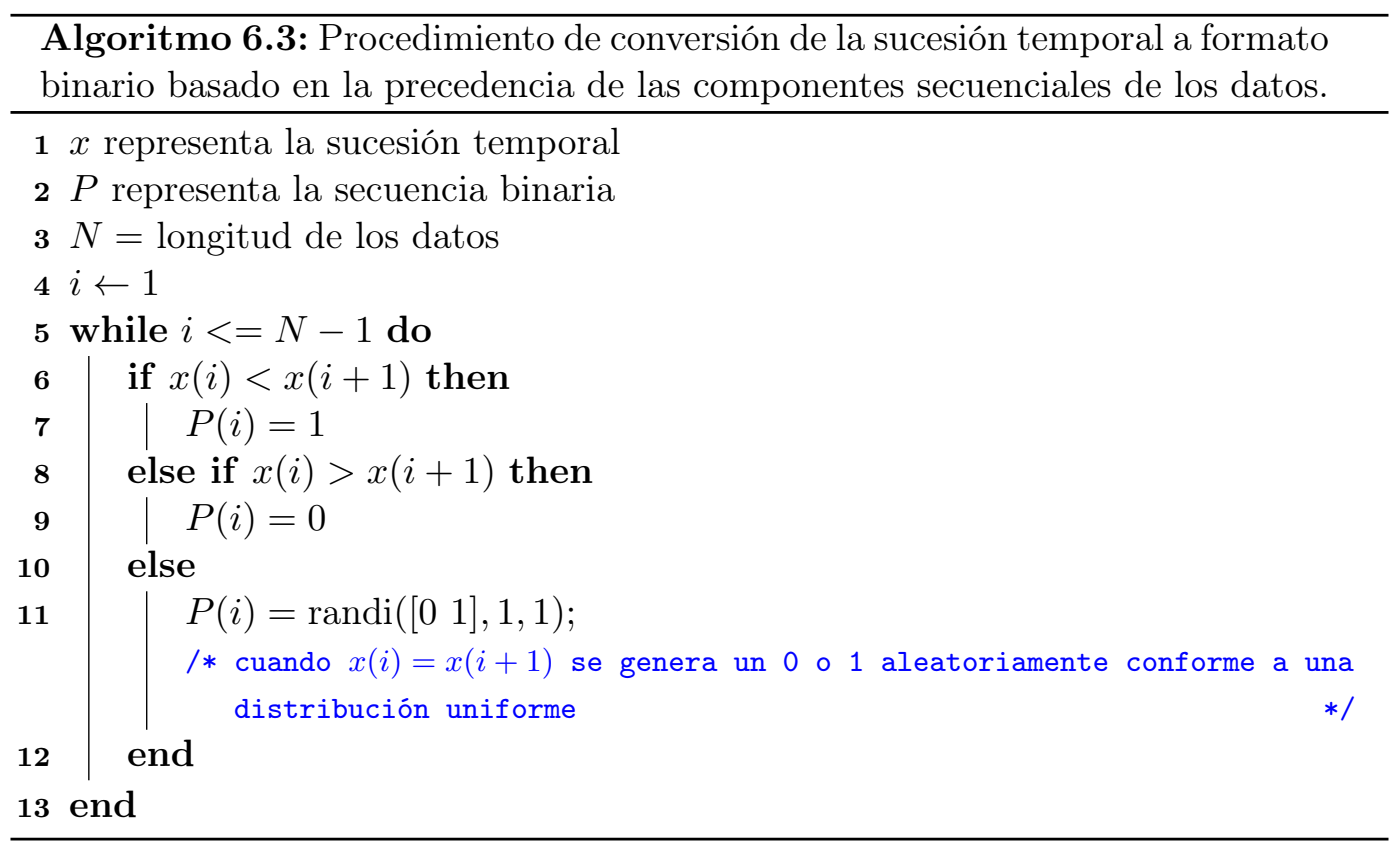

La tabla 6.11 recoge los resultados obtenidos tras aplicar el algoritmo de cálculo de la complejidad de Lempel-Ziv, tanto a las señales de referencia como a las cinco señales PPG, correspondientes a cinco individuos elegidos al azar, una vez convertidas las diferentes sucesiones temporales a sucesiones binarias, de acuerdo con los procedimientos 6.1, 6.2 y 6.3 descritos previamente.

Tabla 6.11: Complejidad de Lempel-Ziv para las diferentes señales de referencia.

\begin{tabular}{|lccc|}
\hline \multicolumn{1}{|c}{ señal evaluada } & algoritmo 6.1 & algoritmo 6.2 & algoritmo 6.3 \\
\hline señal periódica & 0,0037 & 0,0703 & 0,0028 \\
\hline señal cuasiperiódica & 0,1008 & 0,0055 & 0,0832 \\
\hline señal aperiódica & 0,1702 & 0,1054 & 0,1628 \\
\hline señal caótica & 0,5679 & 0,8111 & 0,4356 \\
\hline señal aleatoria & 1,0247 & 0,8268 & 0,9397 \\
\hline individuo núm. 1 (PPG1) & 0,0610 & 0,0842 & 0,0721 \\
\hline individuo núm. 2 (PPG2) & 0,0980 & 0,2136 & 0,1304 \\
\hline individuo núm. 3 (PPG3) & 0,1147 & 0,1341 & 0,1369 \\
\hline individuo núm. 4 (PPG4) & 0,0647 & 0,0943 & 0,1563 \\
\hline individuo núm. 5 (PPG5) & 0,0694 & 0,1397 & 0,1434 \\
\hline \hline
\end{tabular}


De los cambios que se adviertan en la dinámica de las señales PPG, conforme a su evolución temporal y en relación con el dominio examinado, podría establecerse una correspondencia directa entre las variaciones de los patrones que describen la complejidad de su dinámica y aquellos aspectos cualitativos y cuantitativos que determinan la degradación y/o restablecimiento funcional de los múltiples subsistemas físicos constitutivos de los procesos fisiológicos capturados por las señales PPG.

En consecuencia, y especialmente en el ámbito de las señales biológicas, que, en la mayoría de los casos, encierran información relativa a múltiples subsistemas, la transformación aplicada a los datos, a fin de convertir la sucesión temporal original en una sucesión binaria, detenta un protagonismo decisivo. Esto es así porque los diversos subsistemas suelen operar a diferentes escalas y, por consiguiente, la transformación a formato binario puede focalizarse en la respuesta conjunta, o, si fuera preciso, en la respuesta «individual» del subsistema de interés, ${ }^{6}$ es decir, la transformación puede conceder más relieve a una escala que a otra, y, por tanto, pueden derivarse diversas dinámicas, como así quedó recalcado en 6.2.3.3. En cualquier caso, y al margen del algoritmo de transformación, la tabla 6.11 es concluyente en cuanto a que las señales PPG no muestran una complejidad semejante a la de las señales caótica y aleatoria, no así en términos de si aquellas son cuasiperiódicas o aperiódicas.

\footnotetext{
${ }^{6}$ En realidad es prácticamente imposible separar la respuesta íntegra individual de los subsistemas dada la complejidad del acoplamiento mutuo entre ellos.
} 



\section{Referencias}

[Alcaraz, 2018] Alcaraz, R. (2018). Symbolic entropy analysis and its applications. Entropy, 20(8):568. (página 222).

[Allaart and Kawamura, 2011] Allaart, P. and Kawamura, K. (2011). The takagi function: A survey. Exchange, 37:1-54. (página 207).

[Amigó, 2010] Amigó, J. (2010). Permutation Complexity in Dynamical Systems. Springer Berlin Heidelberg. (páginas 217, 218).

[Aravindh et al., 2018] Aravindh, M. S., Venkatesan, A., and Lakshmanan, M. (2018). Strange nonchaotic attractors for computation. Physical Review E, 97(5). (página 206).

[Azami and Escudero, 2018] Azami, H. and Escudero, J. (2018). Amplitude- and fluctuation-based dispersion entropy. Entropy, 20(3):210. (página 211).

[Azami et al., 2017] Azami, H., Rostaghi, M., Abásolo, D., and Escudero, J. (2017). Refined composite multiscale dispersion entropy and its application to biomedical signals. IEEE Transactions on Biomedical Engineering, 64(12):2872-2879. (página 211).

[Bandt and Pompe, 2002] Bandt, C. and Pompe, B. (2002). Permutation entropy: A natural complexity measure for time series. Physical Review Letters, 88(17). (página 217, 217, 217).

[Ben-Mizrachi et al., 1984] Ben-Mizrachi, A., Procaccia, I., and Grassberger, P. (1984). Characterization of experimental (noisy) strange attractors. Physical Review A, 29(2):975-977. (página 213).

[BenSaïda, 2015] BenSaïda, A. (2015). A practical test for noisy chaotic dynamics. SoftwareX, 3-4:1-5. (página 204).

[Costa et al., 2005] Costa, M., Goldberger, A. L., and Peng, C.-K. (2005). Multiscale entropy analysis of biological signals. Physical Review E, 71(2). (página 214).

[Cuesta-Frau et al., 2018] Cuesta-Frau, D., Varela-Entrecanales, M., Molina-Picó, A., and Vargas, B. (2018). Patterns with equal values in permutation entropy: Do they really matter for biosignal classification? Complexity, 2018:1-15. (página 218). 
[Decoster and Mitchell, 1991] Decoster, G. P. and Mitchell, D. W. (1991). The efficacy of the correlation dimension technique in detecting determinism in small samples. Journal of Statistical Computation and Simulation, 39(4):221-229. (página 210).

[du Prel et al., 2009] du Prel, J.-B., Hommel, G., Röhrig, B., and Blettner, M. (2009). Confidence interval or p-value? part 4 of a series on evaluation of scientific publications. Deutsches Aerzteblatt Online. (página 204).

[Eckmann and Ruelle, 1985] Eckmann, J.-P. and Ruelle, D. (1985). Ergodic theory of chaos and strange attractors. Reviews of Modern Physics, 57(3):617-656. (páginas $36,47,105,106,165,213)$.

[Falconer, 1986] Falconer, K. (1986). The Geometry of Fractal Sets. Cambridge Tracts in Mathematics. Cambridge University Press. (página 207).

[Feder, 2013] Feder, J. (2013). Fractals. Physics of Solids and Liquids. Springer US. (página 207).

[Flandrin, 1992] Flandrin, P. (1992). Wavelet analysis and synthesis of fractional brownian motion. IEEE Transactions on Information Theory, 38(2):910-917. (página 207).

[Gopal et al., 2013] Gopal, R., Venkatesan, A., and Lakshmanan, M. (2013). Applicability of 0-1 test for strange nonchaotic attractors. Chaos: An Interdisciplinary Journal of Nonlinear Science, 23(2):023123. (página 222).

[Gottwald and Melbourne, 2004] Gottwald, G. A. and Melbourne, I. (2004). A new test for chaos in deterministic systems. Proceedings of the Royal Society of London. Series A: Mathematical, Physical and Engineering Sciences, 460(2042):603-611. (páginas 135, 220).

[Gottwald and Melbourne, 2005] Gottwald, G. A. and Melbourne, I. (2005). Testing for chaos in deterministic systems with noise. Physica D: Nonlinear Phenomena, 212(1-2):100-110. (páginas 135, 220).

[Gottwald and Melbourne, 2009a] Gottwald, G. A. and Melbourne, I. (2009a). On the implementation of the 0-1 test for chaos. SIAM Journal on Applied Dynamical Systems, 8(1):129-145. (páginas 135, 220).

[Gottwald and Melbourne, 2009b] Gottwald, G. A. and Melbourne, I. (2009b). On the validity of the 0-1 test for chaos. Nonlinearity, 22(6):1367-1382. (páginas 136, 137, 221).

[Gottwald and Melbourne, 2016] Gottwald, G. A. and Melbourne, I. (2016). The 0-1 test for chaos: A review. In Chaos Detection and Predictability, pages 221-247. Springer Berlin Heidelberg. (página 222).

[Graff et al., 2013] Graff, G., Graff, B., Kaczkowska, A., Makowiec, D., Amigó, J., Piskorski, J., Narkiewicz, K., and Guzik, P. (2013). Ordinal pattern statistics for 
the assessment of heart rate variability. The European Physical Journal Special Topics, 222(2):525-534. (página 218).

[Grassberger, 1988] Grassberger, P. (1988). Finite sample corrections to entropy and dimension estimates. Physics Letters A, 128(6-7):369-373. (páginas 67, 122, 213).

[Grassberger and Procaccia, 1983] Grassberger, P. and Procaccia, I. (1983). Estimation of the kolmogorov entropy from a chaotic signal. Physical Review A, 28(4):2591-2593. (páginas 66, 116, 121, 213, 213).

[Grassberger et al., 1991] Grassberger, P., Schreiber, T., and Schaffrath, C. (1991). Nonlinear time sequence analysis. International Journal of Bifurcation and Chaos, 01(03):521-547. (páginas 59, 64, 74, 213).

[Grebogi et al., 1984] Grebogi, C., Ott, E., Pelikan, S., and Yorke, J. A. (1984). Strange attractors that are not chaotic. Physica D: Nonlinear Phenomena, 13(12):261-268. (página 205).

[Higuchi, 1988] Higuchi, T. (1988). Approach to an irregular time series on the basis of the fractal theory. Physica D: Nonlinear Phenomena, 31(2):277-283. (páginas 116, 208).

[Hurst, 1951] Hurst, H. E. (1951). Long-term storage of reservoirs: An experimental study. Transactions of the American Society of Civil Engineers, 116:770-799. (página 208).

[Katz, 1988] Katz, M. J. (1988). Fractals and the analysis of waveforms. Computers in Biology and Medicine, 18(3):145-156. (página 208).

[Keller et al., 2017] Keller, K., Mangold, T., Stolz, I., and Werner, J. (2017). Permutation entropy: New ideas and challenges. Entropy, 19(3):134. (páginas 217, 218).

[Keller et al., 2007] Keller, K., Sinn, M., and Emonds, J. (2007). Time series from the ordinal viewpoint. Stochastics and Dynamics, 07(02):247-272. (página 217).

[Keller et al., 2012] Keller, K., Unakafov, A. M., and Unakafova, V. A. (2012). On the relation of KS entropy and permutation entropy. Physica D: Nonlinear Phenomena, 241(18):1477-1481. (página 217).

[Kolmogorov, 1968] Kolmogorov, A. N. (1968). Three approaches to the quantitative definition of information*. International Journal of Computer Mathematics, 2(14):157-168. (páginas 138, 222).

[Lempel and Ziv, 1976] Lempel, A. and Ziv, J. (1976). On the complexity of finite sequences. IEEE Transactions on Information Theory, 22(1):75-81. (páginas 138, $140,222)$.

[Mesin, 2018] Mesin, L. (2018). Estimation of complexity of sampled biomedical continuous time signals using approximate entropy. Frontiers in Physiology, 9. (páginas 213, 216). 
[Pesin, 1977] Pesin, Y. B. (1977). Characteristic lyapunov exponents and smooth ergodic theory. Russian Mathematical Surveys, 32(4):55-114. (páginas 133, 211).

[Pincus, 1995] Pincus, S. (1995). Approximate entropy (ApEn) as a complexity measure. Chaos: An Interdisciplinary Journal of Nonlinear Science, 5(1):110-117. (página 212).

[Pincus, 1991] Pincus, S. M. (1991). Approximate entropy as a measure of system complexity. Proceedings of the National Academy of Sciences, 88(6):2297-2301. (página 212).

[Prasad et al., 2001] Prasad, A., Negi, S. S., and Ramaswamy, R. (2001). Strange nonchaotic attractors. International Journal of Bifurcation and Chaos, 11(02):291309. (página 206).

[Raghavendra and Dutt, 2010] Raghavendra, B. and Dutt, D. (2010). Computing fractal dimension of signals using multiresolution box-counting method. World Academy of Science, Engineering and Technology, 37:1266-1281. (página 208, 208).

[Ram et al., 2012] Ram, M. R., Madhav, K. V., Krishna, E. H., Komalla, N. R., and Reddy, K. A. (2012). A novel approach for motion artifact reduction in PPG signals based on AS-LMS adaptive filter. IEEE Transactions on Instrumentation and Measurement, 61(5):1445-1457. (página 208).

[Richman and Moorman, 2000] Richman, J. S. and Moorman, J. R. (2000). Physiological time-series analysis using approximate entropy and sample entropy. American Journal of Physiology-Heart and Circulatory Physiology, 278(6):H2039-H2049. (página 213).

[Rostaghi and Azami, 2016] Rostaghi, M. and Azami, H. (2016). Dispersion entropy: A measure for time-series analysis. IEEE Signal Processing Letters, 23(5):610-614. (páginas 211, 212).

[Ruffini et al., 2018] Ruffini, G., Ibañez, D., Kroupi, E., Gagnon, J.-F., Montplaisir, J., Postuma, R. B., Castellano, M., and Soria-Frisch, A. (2018). Algorithmic complexity of EEG for prognosis of neurodegeneration in idiopathic rapid eye movement behavior disorder (RBD). Annals of Biomedical Engineering, 47(1):282296. (página 222).

[Sano and Sawada, 1985] Sano, M. and Sawada, Y. (1985). Measurement of the lyapunov spectrum from a chaotic time series. Physical Review Letters, 55(10):10821085. (páginas 105, 106, 204).

[Sevcik, 2006] Sevcik, C. (2006). On fractal dimension of waveforms. Chaos, Solitons E Fractals, 28(2):579-580. (página 208).

[Shannon, 1948] Shannon, C. E. (1948). A mathematical theory of communication. Bell System Technical Journal, 27(3):379-423. (página 211). 
[Shelhamer, 2007] Shelhamer, M. (2007). Nonlinear Dynamics in Physiology: A State-space Approach. World Scientific. (páginas 161, 204).

[Shumbayawonda et al., 2018] Shumbayawonda, E., Tosun, P., Fernández, A., Hughes, M., and Abásolo, D. (2018). Complexity changes in brain activity in healthy ageing: A permutation lempel-ziv complexity study of magnetoencephalograms. Entropy, 20(7):506. (página 222).

[Stan et al., 2014] Stan, C., Cristescu, C., and Cristescu, C. (2014). Computation of hurst exponent of time series using delayed (log-) returns. application to estimating the financial volatility. UPB Scientific Bulletin, Series A: Applied Mathematics and Physics, 76:235-244. (página 208).

[Sviridova, 2015] Sviridova, N. (2015). Study on chaotic behavior of human photoplethysmogram by comprehensive nonlinear time series analysis. $\mathrm{PhD}$ thesis, Tokyo University of Agriculture and Technology. (páginas 179, 180, 206).

[Sviridova et al., 2018] Sviridova, N., Zhao, T., Aihara, K., Nakamura, K., and Nakano, A. (2018). Photoplethysmogram at green light: Where does chaos arise from? Chaos, Solitons $\&$ Fractals, 116:157-165. (página 222).

[Unakafova and Keller, 2013] Unakafova, V. and Keller, K. (2013). Efficiently measuring complexity on the basis of real-world data. Entropy, 15(12):4392-4415. (páginas 98, 217).

[Zanin et al., 2012] Zanin, M., Zunino, L., Rosso, O. A., and Papo, D. (2012). Permutation entropy and its main biomedical and econophysics applications: A review. Entropy, 14(8):1553-1577. (página 217). 



\section{Capítulo 7}

\section{Conclusiones y líneas abiertas}

En este capítulo final se precisan las conclusiones extraídas una vez evaluados e interpretados los resultados obtenidos del capítulo anterior, el capítulo 6, atendiendo al objetivo inicial, que, según se introdujo en el capítulo 1, aspira a poder caracterizar el comportamiento dinámico de una típica señal PPG de un sujeto joven cualquiera que goce de un buen estado de salud. Con el fin de delimitar la dinámica de una señal PPG se escogieron cinco señales PPG de cinco individuos entresacados al azar y se confrontaron sus respuestas con las exhibidas por señales de referencia con comportamientos conocidos a priori. En todo caso las evoluciones dinámicas de todas ellas, ya sean las señales de referencia o las señales PPG, derivan del despliegue dinámico que proyecta una sucesión temporal escalar, escenario más común en entornos clínicos, cuando a partir de un único observable (los valores sucesivos de la sucesión temporal) se reconstruye el espacio de estados, de acuerdo con el método de los retardos, que presumiblemente describen la funcionalidad del sistema físico del que han sido adquiridas las mediciones. Después de que se dispone de los vectores de estado que configuran los grados de libertad del proceso físico subyacente, se somete al sistema a todo un tratamiento no lineal con vistas a singularizar su complejidad dinámica. Cabe destacar que la reconstrucción del espacio de estados comporta un paso previo imprescindible en el análisis no lineal de sucesiones temporales, de ahí que precisar una metodología sistemática que confiera consistencia a la estimación de la complejidad de una señal biológica sea una pieza clave en este trabajo investigativo. De un examen minucioso de los resultados alcanzados, en consonancia con las dinámicas habituales en señales biológicas procedentes de procesos fisiológicos, se desprenden, a modo de conclusiones, las siguientes reflexiones:

C1 Desde una óptica fisiológica, en el contexto del análisis dinámico de las señales PPG de individuos jóvenes y sanos, se sospecha, cada vez con mayor convencimiento, como así lo evidencian los resultados de los artículos pendientes de publicación (véase el apéndice B), que a pequeña escala prevalece la dinámica cuasiperiódica impuesta por el sistema cardiorrespiratorio. Sin embargo, a gran escala, otros factores relacionados con el sistema cardiovascular entran en juego. Estos factores fuerzan de forma no lineal la dinámica cuasiperiódica y generan dinámicas más complejas y versátiles que contribuyen a un ritmo cardíaco más fácilmente adaptable a las condiciones cambiantes del organismo 
en su relación psicosomática con el entorno.

C2 Resulta notorio que las señales PPG no manifiestan una naturaleza caótica ni aleatoria, con independencia de la técnica de análisis empleada - la complejidad de Lempel-Ziv así lo revela, pues al margen del algoritmo de conversión a formato binario, por muy trivial que sea, las señales PPG no adoptan complejidades próximas a las caóticas o aleatorias - ; por tanto, con este trabajo se descarta la posibilidad, muy extendida en la literatura científica, de que la señal PPG es caótica. En este sentido es probable que la conducta caótica que tan contundentemente sostienen algunos autores se aviene a criterios un tanto subjetivos, esto es, no se reflejan claramente los parámetros utilizados en los métodos analíticos, lo que lleva a sospechar, y ya ciertos gurúes levantaron la voz de alarma hace algunos años, ${ }^{1}$ que «sintonizan» los procedimientos de forma que proporcionen los resultados pretendidos de antemano, desatendiendo la rigurosidad a la par que desestimando el ruido que pudieran contener las señales adquiridas, bien porque los dispositivos son muy sensibles a potenciales perturbaciones, aun siendo involuntarias, o bien porque las señales capturadas no solo describen la dinámica de un único subsistema sino la respuesta combinada de unos cuantos, sincronizados de un modo, en muchos casos, aún desconocido.

C3 El carácter cuasiperiódico o aperiódico de la señal PPG, que no periódico, ${ }^{2}$ no es tan evidente. Discernir entre cuál de las dos respuestas prevalece en la dinámica global del proceso fisiológico latente ha sido uno de los puntos más controvertidos de este trabajo, a tenor de los vagos resultados a este respecto. La mayoría de las dinámicas reflejadas en las señales biológicas reportan la respuesta conjunta de varios subsistemas operando a diferentes escalas espaciotemporales, lo que explicaría, por ejemplo, la naturaleza multifractal de las señales PPG. En consecuencia la secuencia de dimensiones fractales, o código fractal, podría constituir una «huella dactilar» propia e inalienable de cada individuo. De hecho, la entropía de muestra multiescala refleja abiertamente cómo a diferentes escalas el comportamiento dinámico de las señales PPG difiere; a una menor resolución (mayor factor de escala) su dinámica tiende hacia comportamientos caóticos o aleatorios, lo que justificaría la complejidad consustancial al acoplamiento, actuando a bajas frecuencias, entre los subsistemas respiratorio y cardiovascular, particularidad reforzada por el hecho de que el exponente de Hurst adopte estimaciones elevadas, atribuible a procesos persistentes, en sintonía con el espectro de baja frecuencia de un ciclo PPG típico.

C4 Si bien guardan una íntima relación con los valores relativos a una señal cuasiperiódica, que los valores de los exponentes de Lyapunov se sitúen en una

\footnotetext{
${ }^{1}$ Schreiber, T. (1999). Interdisciplinary application of nonlinear time series methods. Physics Reports, 308(1):1-64, consultar pág. 4.

${ }^{2}$ Una inspección preliminar de la densidad espectral de potencia de cualquiera de las cinco señales PPG desecha esta posibilidad, puesto que no se manifiestan armónicos con frecuencias múltiplos enteros de la frecuencia fundamental.
} 
banda muy estrecha alrededor de cero puede ser sintomático de una posible transición al caos, en el caso de que el máximo exponente de Lyapunov se incline hacia valores positivos, o de una transición hacia una dinámica aperiódica representativa de los atractores extraños no caóticos (SNA). Estos atractores contemplan respuestas aperiódicas derivadas de sistemas forzados cuasiperiódicamente, lo que, por un lado, respaldaría la naturaleza fractal de las señales PPG, y, por otro lado, que en algunos tests, como sucede con las entropías de dispersión, aproximada o de permutación, sea arduo elucidar cuál de las dos respuestas predomina con claridad palmaria.

C5 El modelo difusivo de las señales PPG, conforme al test $0-1$, de por sí muy confinado en el espacio, presenta en todas las trazas geométricas una región central que articula una disposición transitiva, superpuesta a una dinámica «periódica», dado el carácter recurrente de los ciclos PPG, pasando por estadíos aperiódicos, conforme se aleja la configuración del centroide estructural, que en su posible transición hacia el caos se ve detenida por la acción cuasiperiódica, característica de un atractor SNA, en el cinturón perimetral. La apertura más o menos aperiódica, controlada por la actuación cuasiperiódica, pone de manifiesto una mecánica difusiva que combina ambas dinámicas funcionales, en las que a veces el engranaje operativo, siempre confinado en el espacio, concede más margen al extremo aperiódico.

\subsection{Líneas abiertas}

Entre las líneas de investigación abiertas, esencialmente en el contexto de las señales PPG, promovidas por los resultados de este trabajo, sobresalen los siguientes itinerarios:

L1 Definir indicadores cuantitativos del estado fisiológico de un individuo, sobre la base de parámetros dinámicos de la señal PPG introducidos en este trabajo, a fin de poder establecer un diagnóstico precoz de anomalías pasajeras, como un episodio de estrés, o incluso de afecciones más graves, como infartos de miocardio.

L2 Extender la reconstrucción del espacio de estados a otras metodologías menos empíricas de pronunciado carácter probabilístico, como el estadístico que evalúa las relaciones funcionales entre las componentes de cada vector de estado, de forma que cada vector reconstruido retenga la transferencia de información que se intercambian los subsistemas a diferentes escalas.

L3 Estimar el espectro de dimensiones de Rényi de las señales PPG hasta un orden determinado, en función de los datos disponibles, evaluándose los marcadores particulares de cada individuo y cómo estos sellos distintivos pueden verse alterados por patologías físicas o emocionales (estrés).

L4 Examinar, a partir del exponente de Hurst, la volatilidad que experimentan los ciclos PPG cuando los individuos son sometidos a situaciones estresantes. 
L5 Evaluar los cambios que experimentan las entropías de dispersión, aproximada, de muestra multiescala y de permutación en los diferentes ciclos PPG adquiridos por un oxímetro de pulso, cuando los individuos son sometidos a situaciones estresantes. Cambios significativos en las diferentes ventanas temporales podrían desvelar variaciones en la respuesta funcional de los subsistemas fisiológicos implicados, lo que llevaría a intentar establecer vínculos con los parámetros físicos que provocan dicha alteración.

L6 Modelizar un ciclo PPG a partir de un mapa de la circunferencia, de manera que pueda estudiarse el impacto que provocan los ruidos, acoplados más frecuentemente en el proceso de medición de señales PPG, en su dinámica. Además, el modelo de ciclo PPG fundamentado en los mapas de la circunferencia permitiría evaluar la transición al caos desde un comportamiento cuasiperiódico o, al menos, estudiar en profundidad los ingredientes que intervienen en un atractor extraño no caótico (SNA) y qué implicaciones tienen desde un punto de vista funcional en el comportamiento de una señal PPG en un estado basal y en un estado estresado. El análisis de un SNA puede complementarse mediante los gráficos de recurrencia (recurrence plots) o el propio test $0-1$.

L7 Analizar la estructura geométrica del modelo de difusión típico de una señal PPG, de acuerdo con el test $0-1$, determinándose tanto los parámetros geométricos característicos de cada individuo, esto es, que se mantienen constantes con independencia de los cambios de estado que pudiera sufrir el sujeto, como aquellas variables que, en presencia de situaciones estresantes o de patologías más graves, experimentan cambios significativos. Los sistemas hamiltonianos controlados por puertos (port-Hamiltonian systems) pueden arrojar algo de luz en cuanto a la dinámica de la entropía, en términos energéticos, cuando diversos subsistemas son interconectados entre sí.

L8 Examinar la función de transformación binaria utilizada en el análisis de complejidad de Lempel-Ziv, de modo que incorpore los cambios que se observan en las fases de los coeficientes de Fourier - incluso hacer un seguimiento de la dinámica de las fases del mapa de la circunferencia-, en la primera y/o en la segunda derivada de la señal PPG. En este sentido los algoritmos derivados de los grafos de visibilidad (visibility graphs) también desempeñan un papel digno de ser explorado en profundidad.

L9 Definir mediante la entropía de correlación del espacio de estados la matriz de ponderación de un comportamiento histerético basado en un modelo de Preisach. De este modo podrían analizarse características dinámicas de la señal PPG desde otra perspectiva totalmente nueva, que, si fuere el caso, se convertirían en señas de identidad de un sujeto (semilla de un potencial algoritmo de cifrado de información) o en indicadores de diagnóstico de posibles anomalías funcionales de la señal PPG, consecuencia de, por ejemplo, disfunciones transitorias tras situaciones estresantes.

L10 Diseñar un sistema de control basado en modelos estocásticos, de suerte que con la información probabilística del modelo, generado a partir de las muestras 
de la señal PPG, pueda controlarse, una vez comprendido el acoplamiento entre las piezas que conforman la funcionalidad de un ciclo PPG, el estado de excitación y/o recuperación de la dinámica de este tipo de señales biológicas. 



\section{Apéndice A}

\section{Señales de referencia y equipamiento destinado}

En este apéndice se describen las señales de referencia que se han utilizado en este trabajo, así como el equipamiento necesario para diseñar y ejecutar los algoritmos con los que se recabaron los valores numéricos presentados.

\section{A.1 Señales de referencia}

\section{A.1.1 Señales de referencia básicas}

Las cinco señales de referencia básicas abarcan un espectro de gran amplitud, desde las señales más regulares o periódicas a las más irregulares o aleatorias.

1. Señal periódica. Onda de sierra operando a una frecuencia $f=10 \mathrm{~Hz}$.

$$
y_{\text {periódica }}(t)=2\left(\frac{t}{1 / f}-\left\lfloor\frac{1}{2}+\frac{t}{1 / f}\right\rfloor\right) \text {. }
$$

2. Señal cuasiperiódica. La relación entre las frecuencias es irracional; en este caso se opta por una configuración muy simple, basada en dos ondas sinusoidales de frecuencias respondiendo a una relación irracional de $1 / \sqrt{2}$.

$$
y_{\text {cuasiperiódica }}(t)=\sin (2 \pi 10 \cdot t)+\sin (2 \pi 10 \sqrt{2} \cdot t) .
$$

3. Señal aperiódica. De período infinito, por medio de un barrido lineal de frecuencias de la función chirp, a saber, $f(t)=f_{0}+k \cdot t$, entre $f_{0}=0 \mathrm{~Hz} \mathrm{y}$ $f_{1}=10 \mathrm{~Hz}$, con $k=\left(f_{1}-f_{0}\right) / T$, siendo $T$ el tiempo de barrido y $f_{s}=250 \mathrm{~Hz}$ la frecuencia de muestreo.

$$
y_{\text {aperiódica }}(t)=\sin \left[2 \pi\left(f_{0} t+\frac{k}{2} t^{2}\right)\right] \text {. }
$$


4. Señal caótica. A partir del mapa de Hénon se emplea la variable dinámica $x_{n}$, con $a=1,4$ y $b=0,3$. Las condiciones iniciales obedecen a $x_{0}=y_{0}=0,03$. Se generan 100.000 muestras, de las que se descartan todos los puntos a excepción de los últimos 15.000 elementos.

$$
\begin{aligned}
x_{n+1} & =1-a x_{n}^{2}+y_{n} \\
y_{n+1} & =b x_{n} .
\end{aligned}
$$

5. Señal aleatoria. Se generan 15.000 puntos, extraídos de una distribución uniforme (caracterización probabilística) restringida al intervalo $[0,1]$.

\section{A.1.2 Señales PPG}

Las señales PPG proceden de un proyecto tutelado por el grupo de investigación Tecnologías de Apoyo a la Vida - en inglés Life Supporting Technologies - , perteneciente a la ETS de Ingenieros de Telecomunicación de la Universidad Politécnica de Madrid (https://ww. Ist.tfo.upm.es), proyecto ES3: intentar cuantificar $y$ medir el nivel de estrés, ${ }^{1}$ en el que se adquirieron, entre otras, medidas de la señal PPG de cuarenta individuos con edades comprendidas entre los dieciocho y los veinticinco años. De estos cuarenta sujetos, para este trabajo, se escogieron cinco muestras de la señal PPG al azar.

\section{A.2 Equipamiento destinado}

El equipamiento dedicado a este trabajo engloba un entorno de desarrollo software, para la programación algorítmica, y una infraestructura hardware, donde se ejecutan los programas que implementan la algoritmia.

\section{A.2.1 Entorno de desarrollo software}

El siguiente listado inventaría las aplicaciones software utilizadas tanto para programar los algoritmos matemáticos como para procesar y reproducir las figuras de la memoria, publicada en $\mathrm{LAT}_{\mathrm{E}} \mathrm{X}$ mediante el editor TeXShop para macOS.

1. Software MATLAB ${ }^{\circledR}$ R2017b (9.3.0.713579) para macOS. Número de licencia desde 2017: 40496933.

2. TISEAN` 3.0.1 Nonlinear Time Series Analysis para UNIX ${ }^{\circledR}$.

3. gnuplot 5.2 para Linux.

4. Xfig 3.2.6 para Linux.

5. AWK versión 20070501 para macOS.

\footnotetext{
${ }^{1}$ Aguiló, J., Ferrer-Salvans, P., García-Rozo, A., Armario, A., Corbi, A., Cambra, F. J., Bailón, R., González-Marcos, A., Caja, G., Aguiló, S., López-Antón, Arza-Valdés, A., and Garzón-Rey, J. M. (2015). Project ES3: Attempting to quantify and measure the level of stress. Revista de neurología, 61:405-415.
} 


\section{A.2.2 Infraestructura hardware}

A continuación se especifican las características técnicas de las arquitecturas hardware empleadas en este trabajo.

1. MacBook Air personal de 13 pulgadas, principios de 2015. Procesador $1,6 \mathrm{GHz}$ Intel Core i5. Memoria 8 GB $1600 \mathrm{MHz}$ DDR3. Gráficos Intel HD Graphics 60001536 MB. Sistema operativo macOS Sierra versión 10.12.3 (16D32).

2. Cluster de computación del Grupo de Investigación en Computación de Altas Prestaciones de la Universidad de Alcalá (http://hpc.aut.uah.es), localizado en la Escuela Politécnica Superior. El cluster consta de diez nodos de computación, con un total de 64 núcleos y arquitectura Intel Xeon, y 166 GB de memoria. Un nodo frontend centraliza la gestión de la ejecución de los trabajos a través del sistema SLURM. Además, se dispone de servidores de discos compartidos que utilizan la tecnología NFS clásica y BeeGFS, sistema de ficheros distribuido especializado para computación de altas prestaciones, con lo que se alcanza un total de 1,5 TB de almacenamiento compartido. Cada nodo dispone así mismo de una zona de disco local para almacenamiento temporal a disposición de las aplicaciones. Todos los nodos utilizan Linux Debian en su última distribución estable y disponen de diversas bibliotecas de programación y software licenciado. 



\section{Apéndice B}

\section{Publicaciones en revistas indexadas}

Las publicaciones que avalan la calidad de este trabajo se detallan a continuación.

- Aceptada para su publicación [cuartil Q1]. de Pedro-Carracedo J, FuentesJimenez D, Ugena AM, Gonzalez-Marcos AP. Phase Space Reconstruction from a Biological Time Series: A Photoplethysmographic Signal Case Study. Applied Sciences. 2020; 10(4):1430. https://doi.org/10.3390/app10041430.

- Pendientes de publicación [cuartil Q1].

1. J. de Pedro-Carracedo, Iván García Daza, A.M. Ugena, and A.P. GonzalezMarcos, Dynamical analysis of biological signals with the 0-1 test. A PhotoPlethysmoGraphic signal a case study. Preprint available at arXiv.org, file reference: arXiv:1906.00782. [https://arxiv.org/abs/1906.00782].

2. J. de Pedro-Carracedo, D. Fuentes-Jimenez, A.M. Ugena, and A.P. Gonzalez-Marcos, Is the PPG signal chaotic? Preprint available at arXiv.org, file reference: arXiv:2002.01054. [https://arxiv.org/abs/2002.01054]. 